NORTHWESTERN UNIVERSITY

\title{
A Study of Two Photon Decays of Charmonium Resonances Formed in Proton-Antiproton Annihilations
}

\author{
A DISSERTATION \\ SUBMITTED TO THE GRADUATE SCHOOL \\ IN PARTIAL FULFILMENT OF THE REQUIREMENTS \\ for the degree \\ DOCTOR OF PHILOSOPHY
}

Field of Physics

By

Todd K. Pedlar

EVANSTON, ILLINOIS

June 1999 
(C) by Todd Kristofer Pedlar 1999

All Rights Reserved 


\title{
Abstract of the Dissertation
}

\section{A Study of Two Photon Decays of Charmonium Resonances Formed in Proton-Antiproton Annihilations}

\author{
by \\ Todd Kristofer Pedlar \\ Doctor of Philosophy in Physics \\ Northwestern University, 1999 \\ Professor Kamal K. Seth, Chair
}

In this dissertation we describe the results of an investigation of the production of charmonium states $\left(\eta_{c}, \eta_{c}^{\prime}, \chi_{0}\right.$ and $\left.\chi_{2}\right)$ in Fermilab experiment E835 via antiprotonproton annihilation and their detection via their decay into two photons. The $\eta_{c}$ resonance parameters were determined to be $M\left(\eta_{c}\right)=2982.4_{-2.2}^{+2.3} \mathrm{MeV}, \Gamma\left(\eta_{c}\right)=$ $26.9_{-9.5}^{+10.8} \mathrm{MeV}$ and $\Gamma\left(\eta_{c} \rightarrow \gamma \gamma\right)=5.7_{-2.3-1.4}^{+2.9+2.9} \mathrm{keV}$. For the $\chi_{2}$ resonance, a partial width $\Gamma\left(\chi_{2} \rightarrow \gamma \gamma\right)=0.343_{-0.051-0.038}^{+0.053+0.043} \mathrm{keV}$ was measured. No evidence in the $\gamma \gamma$ decay mode for either $\chi_{0}($ near $\sqrt{s} \simeq 3415 \mathrm{MeV})$ or $\eta_{c}^{\prime}$ (in the region $\left.\sqrt{s}=3575-3660 \mathrm{MeV}\right)$ regions was found. $90 \%$ confidence upper limits were established at $\Gamma\left(\chi_{0} \rightarrow \gamma \gamma\right) \leq 1.3$ $\mathrm{keV}$, and $B\left(\eta_{c}^{\prime} \rightarrow \bar{p} p\right) \times B\left(\eta_{c}^{\prime} \rightarrow \gamma \gamma\right) \leq 5.9 \times 10^{-8}$. 
To Heather, for all her support. 


\section{Acknowledgements}

First of all, I thank my advisor, Kamal K. Seth for all his guidance and advice throughout this project, and the seven years of our working relationship. He cracked the whip when necessary, pointed out where things needed work, but also found the time to let me know when I did things right.

I would like to thank my comrades-in-arms in Kam's group: former students Camille Ginsburg and Steve Trokenheim, current student David Joffe, and all the post-docs I have worked with: Miguel Sarmiento, David Brown, Xiaoling Fan and Amiran Tomaradze. Their support and friendship will be appreciated always.

Thanks to all the grad students who made life more enjoyable during my time at Northwestern; to Tracy Taylor Thomas, Chris Thomas, and Mary Durbin especially, for their companionship during our years together at NU. Bridge parties and allnight quantum theory jams will always be remembered. Without comaraderie we shared, I don't know how any of us would have made it through - especially that 
dreaded Finn-Sauls-Garg quarter.

To all my collaborators on E835, and especially our spokespeople, Stephen Pordes and Rosanna Cester, my heartfelt thanks and best wishes. I sincerely hope we get the chance to work together again in pursuit of good physics.

I would like to thank my professors at Whitman College for their teaching and dedication to my education there. All of them contributed in a big way to my development as a well-rounded student of physics and mathematics. Thanks in particular to Jim Pengra, Craig Gunsul, Phil Howland, Robert Blumenthal, Larry Anderson, Dave Guichard, and Bob Fontenot.

I would also like to thank my parents, for their constant encouragement during my upbringing; for always supporting me in everything I did, whether it be soccer, basketball, baseball, or school - in all things I was given the confidence to succeed, and the consolation when things didn't go so well.

And to Heather - through the good and bad she always stuck by me, and constantly reminded me that there was more to life the physics. Her support during the data-taking phase of this experiment, and especially during the long period of writing this dissertation was invaluable, and for that I owe her my deepest gratitude. 


\section{Contents}

Abstract of the Dissertation

Acknowledgements $\quad$ v

List of Figures $\quad$ xxxii

List of Tables $\quad$ xxxvii

1 Introduction 1

2 Charmonium - a Theoretical Background 4

2.1 The Quark Model and the Origins of QCD . . . . . . . . . . . 4

2.2 Potential Models for the Strong Interaction . . . . . . . . . . . . 7

2.3 Quantum Electrodynamics and Positronium ........... 11

2.3.1 Application of QED to positronium decays . . . . . . . . 13

2.4 Quantum Chromodynamics .............. 15 
2.5 Application of QED and QCD to charmonium decays . . . . . . 16

3 Experimental Study of Charmonium 20

3.1 Why charmonium? ................... 20

3.2 Discovery of Charmonium . . . . . . . . . . . . . . 22

3.3 Charmonium Production in $e^{+} e^{-}$collisions . . . . . . . . . . 24

3.4 Charmonium Production in $\bar{p} p$ collisions . . . . . . . . . . . . . 27

4 Fermilab Experiment E835 30

$4.1 \bar{p}$ Production, Cooling and Accumulation . . . . . . . . . . . 30

4.1.1 Transverse Stochastic Cooling . . . . . . . . . . . 34

4.1.2 Longitudinal Stochastic Cooling . . . . . . . . . . 35

4.1.3 $\bar{p}$ Stacking in the Accumulator - Preparations for Resonance

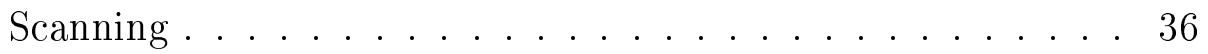

$4.2 \bar{p}$ Beam Energy Measurement . . . . . . . . . . . . . . . 40

4.3 Hydrogen Gas Jet Target . . . . . . . . . . . . . . . . . 45

4.4 The E835 Detector . . . . . . . . . . . . . . . 49

4.4 Inner detectors ................... . 49

4.4 Čerenkov Detector ................. 57

4.4.3 Central Calorimeter . . . . . . . . . . . 60

4.4.4 Luminosity Monitor ............... 70

viii 
$\begin{array}{lll}5 & \text { Data Acquisition } & 76\end{array}$

5.1 DAQ Hardware . . . . . . . . . . . . . . 76

5.2 E835 Trigger Scheme . . . . . . . . . . . . . . 78

5.3 The Charged Trigger . . . . . . . . . . . . . . . 79

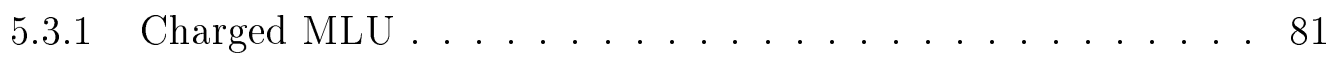

5.4 The Neutral Trigger ................... . . 84

5.4 Level I Summers . . . . . . . . . . . . . 88

5.4 Level II Summers . . . . . . . . . . . . . . . . 88

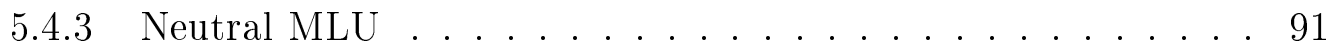

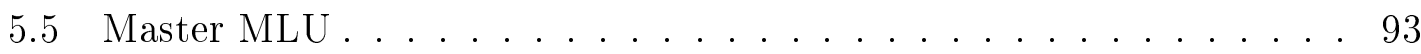

5.6 Other Triggers . . . . . . . . . . . . . . 97

5.6.1 Minimum Bias .................. 97

5.6 .2 Laser Monitor . . . . . . . . . . . . . . 97

5.6 .3 Random Gate . . . . . . . . . . . . . 97

5.7 The Gatemaster . . . . . . . . . . . . . . . 98

5.8 Software Trigger Level: PRUDE . . . . . . . . . . . . . 99

5.9 Luminosity DAQ . . . . . . . . . . . . . . 104

6 Identification of Photons 106

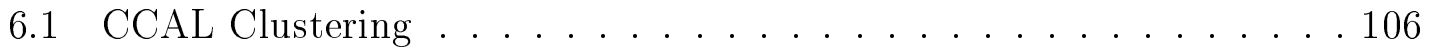


6.1.1 Main Clustering Algorithm ............. 107

6.1.2 Isolated Cluster Analysis . . . . . . . . . . . . . 110

6.1.3 Non-Isolated Cluster Analysis . . . . . . . . . . . . 113

6.1.4 Cluster Splitting Analysis ............. 115

6.2 Photon Identification . . . . . . . . . . . . . . 115

6.2.1 Possible Misidentification of $\gamma^{\prime}$ s . . . . . . . . . 116

6.2.2 Inefficiency due to $\gamma$ conversions . . . . . . . . . . . 117

7 Selection and Analysis of $\gamma \gamma$ Events 123

7.1 Initial Data Selection . . . . . . . . . . . . . . . 123

$7.2 \gamma \gamma$ Event Selection Criteria . . . . . . . . . . . . 125

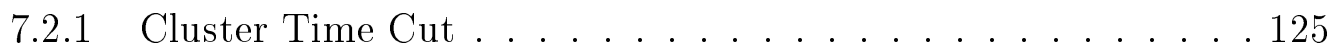

7.2.2 Invariant mass between $\gamma$ candidate cluster and "extra" clus-

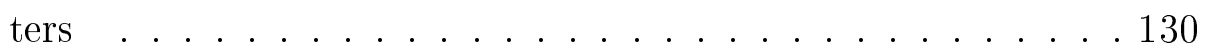

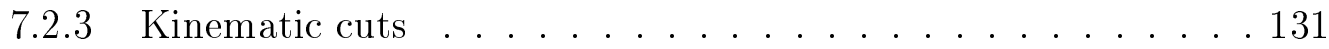

7.2.4 Effect of "Noise" and Extraneous Interactions on Efficiency . 136

7.2.5 Polar Angle $\theta$ of the $\gamma$ candiates. . . . . . . . . . . 139

7.2.6 Charged Track Contamination at $J / \psi$ and $\psi^{\prime} \ldots 143$

7.3 Overall Efficiency of Cuts ................... 146 
8.1 Background contributions to $(c \bar{c})_{R} \rightarrow \gamma \gamma \ldots \ldots \ldots 151$

8.2 Determination of Resonance and Background Parameters . . . . . . 163

8.3 Resonance Parameters of $\eta_{c} \ldots \ldots$. . . . . . . . . . 164

8.3.1 The $\eta_{c}$ Mass . . . . . . . . . . . . . . . 168

8.3.2 The Total Width of $\eta_{c} \ldots \ldots$. . . . . . . . . . 172

8.3.3 The Two Photon Partial Width of $\eta_{c} \ldots . . . . . . .175$

8.4 The Two Photon Partial Width of $\chi_{2} \quad \ldots . . . . . . . . . .180$

8.5 Measurement of $\bar{p} p \rightarrow \chi_{0} \rightarrow \gamma \gamma \ldots \ldots \ldots \ldots$

8.6 The Search for $\eta_{c}^{\prime} \ldots \ldots \ldots$. . . . . . . . . . . . . . . . . .

8.7 Summary of Results . . . . . . . . . . . . . . 198

8.8 Discussion of Results . . . . . . . . . . . . . . . 198

9 Determination of $\alpha_{\mathrm{S}} \quad 205$

$9.1 \alpha_{\mathrm{S}}$ determined by $\eta_{c}$ data $\ldots \ldots \ldots \ldots$. . . . . . . . . . . . . . .

$9.2 \alpha_{\mathrm{S}}$ as determined by $\chi_{2}$ data . . . . . . . . . . . 208

9.3 Comparison to Other Results for $\alpha_{\mathrm{S}} \ldots \ldots$. . . . . . . 209

10 Comments and Reflections $\quad 213$

$\begin{array}{ll}\text { Bibliography } & 215\end{array}$ 
A The CCAL Splitter-Shaper-Discriminator Circuit

A.1 Introduction . . . . . . . . . . . . . . . . . 224

A.2 Testing of the SSD Prototype . . . . . . . . . . . 225

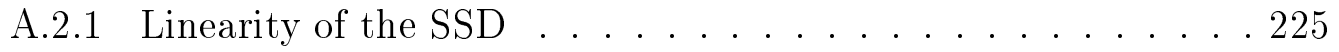

A.2.2 Shaped Pulses and Pileup . . . . . . . . . . . 228

A.3 Timing of Shaped Pulses . . . . . . . . . . . . . . . . 230

A.4 Beam Test at Brookhaven . . . . . . . . . . . . . 230

B Performance of the Central Calorimeter (CCAL) 234

B.1 Angular Resolutions . . . . . . . . . . . . . . . . 235

B.2 Energy Resolution . . . . . . . . . . . . . . . . . . . 242

B.3 CCAL Gain Variation . . . . . . . . . . . . . . 245

B.3.1 Laser Monitoring . . . . . . . . . . . . . . . . . . . 245

B.3.2 Gain Constant Calculation . . . . . . . . . . . . . . 246

C Angular Distribution of the Reactions $\bar{p} p \rightarrow \eta_{c} \rightarrow \gamma \gamma$ and $\bar{p} p \rightarrow \chi_{2} \rightarrow \gamma \gamma 249$

D Statistical Considerations 255

E Summary of All $\gamma \gamma$ Data $\quad 265$ 


\section{List of Figures}

$2.1 \mathrm{SU}(3)$ classification of light quarks and hadrons. . . . . . . . . 5

2.2 A schematic showing the positronium and charmonium spectra. . . 8

2.3 Schematic showing various model potentials. . . . . . . . . . 9

2.4 Feynman diagrams illustrating the basic QED vertex . . . . . . 11

2.5 The Feynman diagrams for the 2-photon annihilation process. . . . . 12

2.6 The three basic QCD vertices. ............... 16

3.1 Spectra demonstrating the first experimental evidence for $J / \psi \ldots$. . 23

3.2 The spectrum of charmonium states. . . . . . . . . . . 25

3.3 Formation of vector charmonium states via $e^{+} e^{-}$annihilation. . . . . 26

3.4 Formation of charmonium states via $\bar{p} p$ annihilation. . . . . . . . . 28

4.1 Schematic of the Fermilab Antiproton Source. . . . . . . . . . . 31

4.2 Schematic of the antiproton production target area. . . . . . . 32

4.3 Schematic showing RF manipulations of $\bar{p}$ bunches. . . . . . . . . . 33 
4.4 Schematic of a stochastic cooling system. . . . . . . . . . . . 34

4.5 Schematic showing the cycle of $\bar{p}$ stacking. . . . . . . . . . . 37

4.6 Hypothetical scan of a resonance. . . . . . . . . . . . . . 39

4.7 Schematic illustrating a single particle in a storage ring. . . . . . . . . 41

4.8 Semilog plot of the Schottky frequency distribution from an E835 run. 43

4.9 Schematic illustration of the E835 Gas Jet target. . . . . . . . . 47

4.10 Measured gas jet density distribution. . . . . . . . . . . . 48

4.11 Schematic of the E835 detector system . . . . . . . . . . 50

4.12 Schematic of the inner detectors. . . . . . . . . . . . 51

4.13 Pulse height spectra from hodoscopes $H 1, H 2^{\prime}$ and $H 2$. . . . . . 53

4.14 Pulse height spectrum for hodoscope $H 2 \ldots$. . . . . . . . . . . . 56

4.15 Schematics of the Čerenkov detector. . . . . . . . . . . 58

4.16 Normalized signal from the Čerenkov counter. . . . . . . . . . . 59

4.17 Efficiency for Čerenkov detection of single electrons. . . . . . . . . . . 60

4.18 Schematic of the CCAL, azimuthal view. . . . . . . . . . . 62

4.19 Schematic of the CCAL, lateral view. Two wedges are shown. . . . . 62

4.20 Schematic showing the internal construction of a CCAL wedge. . . . 64

4.21 Schematic diagram of an SSD channel. . . . . . . . . . . 66

4.22 CCAL PMT pulses at the input and output of the SSD. . . . . . 67 
4.23 Schematics of the CCAL laser monitor system. . . . . . . . . . 69

4.24 Comparison of cosmic ray and laser signals. . . . . . . . . . . . 71

4.25 Schematic of the E835 Luminosity Monitor detector pan. . . . . . . . 73

4.26 Schematic of the E835 Luminosity Monitor showing the three detectors. 73

4.27 Examples of proton recoil spectra from the LM. . . . . . . . . . 75

5.1 Schematic of the E835 Data Acquisition System. . . . . . . . . . 77

5.2 Schematic showing the process of E835 trigger construction. . . . . 82

5.4 Schematic of the E835 Neutral Trigger. . . . . . . . . . . 85

5.5 Rollout diagram of the 1280 CCAL elements. . . . . . . . . . 87

5.6 Illustration of two-body trigger threshold settings. . . . . . . . . . 90

5.7 Neutral MLU inputs and outputs. . . . . . . . . . . . . 92

5.8 Output from the luminosity program from one of the E835 $\mathrm{J} / \psi$ runs. 105

6.1 Depiction of isolated and non-isolated cluster seeds. . . . . . . . . . 109

6.2 Comparison of cluster masses for electrons and for neutral clusters. . 111

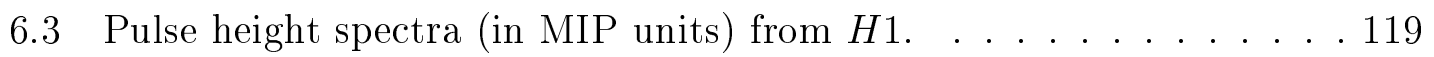

6.4 Pulse height spectra (in MIP units) from $H 2^{\prime}$. . . . . . . . . . 120

6.5 Pulse height spectra (in MIP units) from H2. . . . . . . . . . . 120

6.6 Pulse height spectra (in electron units) from the Čerenkov counter. . 122

7.1 Invariant mass combinations for events in the "gold gg" trigger. . . . 124 
7.2 Cluster times for clean $\pi^{0} \pi^{0}$ events. . . . . . . . . . . . 126

7.3 Number of clusters for events passing all cuts but the timing cut. . . 128

7.4 Number of CCAL clusters for events passing all cuts. . . . . . . . 128

7.5 Fraction of clusters which have timing information. . . . . . . . 132

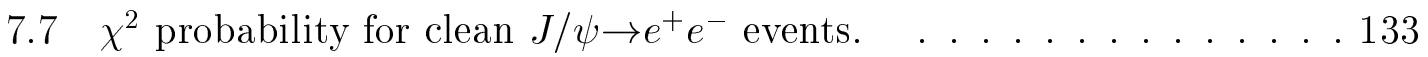

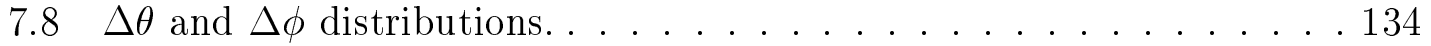

7.9 Luminosity dependence of efficiencies. . . . . . . . . . . . . 138

7.10 Dependence of the $5 \% \chi^{2}$ probability cut on $\sqrt{s} \ldots \ldots \ldots \ldots$

$7.12 \pi^{0} \pi^{0}$ and $\pi^{0} \gamma$ angular distributions and $\gamma \gamma$ candidate events at $\eta_{c} . \quad .141$

$7.12 \pi^{0} \pi^{0}$ and $\pi^{0} \gamma$ angular distributions and $\gamma \gamma$ candidate events at $\eta_{c}^{\prime} . \quad 142$

7.13 Azimuthal angle $\phi_{1,2}$ for neutral trigger events. . . . . . . . . 146

$8.1 \gamma \gamma$ Cross Sections for (top) $\left|\cos \theta^{*}\right| \leq 0.2$ and (bottom) $\left|\cos \theta^{*}\right| \leq 0.4 .148$

$8.2 \gamma \gamma$ Cross Sections for $\left|\cos \theta^{*}\right| \leq 0.2 \ldots \ldots \ldots \ldots \ldots \ldots$

$8.3 \gamma \gamma$ Cross Sections for $\left|\cos \theta^{*}\right| \leq 0.4 \ldots \ldots \ldots \ldots \ldots \ldots$

8.4 Calculated feed-down cross sections based on E760 measurements. . . 152

8.5 Measured cross sections for the reaction $\gamma \gamma \rightarrow \bar{p} p$ for $\left|\cos \theta^{*}\right| \leq 0.6$. . 154

8.6 Measured differential cross section for the reaction $\gamma \gamma \rightarrow \bar{p} p \ldots \ldots \ldots$

8.7 Results of two fits to the E760 $\gamma \gamma$ cross sections. . . . . . . . . 158

$8.8 \gamma \gamma$ Cross Sections for $\left|\cos \theta^{*}\right| \leq 0.2$, for 2900 to $3400 \mathrm{MeV} \ldots \ldots . .160$ 
8.9 Results of fitting the background to the data for $\left|\cos \theta^{*}\right| \leq 0.2 \ldots \ldots 2$

8.10 Measured cross sections for $\bar{p} p \rightarrow \gamma \gamma$ for $\left|\cos \theta^{*}\right| \leq 0.2$ in the $\eta_{c}$ region. 165

8.11 Results of a study of the stability of the results for $\eta_{c}$. . . . . . . 169

8.12 Results of measurements of the $\eta_{c}$ mass. . . . . . . . . . . 170

8.13 Experimental measurements of the $\eta_{c}$ total width. . . . . . . . 173

8.14 Inclusive photon spectra from $J / \psi \rightarrow \gamma X$ obtained by the Crystal Ball. 174

8.15 Experimental measurements of $\Gamma\left(\eta_{c} \rightarrow \gamma \gamma\right) \ldots \ldots . \ldots . \ldots 177$

8.16 Invariant mass spectra from CLEO. . . . . . . . . . . . 178

8.17 Measured cross sections for $\bar{p} p \rightarrow \gamma \gamma$ for $\left|\cos \theta^{*}\right| \leq 0.4$ in the $\chi_{2}$ region. 181

8.18 Results of a study of the stability of the result for $\Gamma\left(\chi_{2} \rightarrow \gamma \gamma\right) . \ldots 183$

8.19 Experimental measurements of $\Gamma\left(\chi_{2} \rightarrow \gamma \gamma\right) \ldots \ldots . . \ldots 184$

8.20 Measured cross sections for $\bar{p} p \rightarrow \gamma \gamma$ for $\left|\cos \theta^{*}\right| \leq 0.4$ near $\chi_{0}$. . . 186

8.21 Evidence for $\eta_{c}^{\prime}$ from the Crystal Ball. . . . . . . . . . . . . 187

$8.2290 \%$ CL upper limits for $B\left(\eta_{c}^{\prime} \rightarrow \bar{p} p\right) \times B\left(\eta_{c}^{\prime} \rightarrow \gamma \gamma\right)$ from E760. . . . 192

8.23 Measured cross sections for $\bar{p} p \rightarrow \gamma \gamma$ for $\left|\cos \theta^{*}\right| \leq 0.4$ near $\eta_{c}^{\prime}$. . . . 193

8.24 Cross sections and branching fraction product upper limits for $\eta_{c}^{\prime}$. . . 194

8.25 The sum of invariant mass distributions from DELPHI. . . . . . . . . 197

9.1 Measurements of $\alpha_{\mathrm{S}}(Q)$ from various experiments. . . . . . . . . 212

A.1 Schematic diagram of an SSD channel. . . . . . . . . . . . 226

xvii 
A.2 Test setup for the SSD prototype at FNAL. . . . . . . . . . 227

A.3 Result of linearity test for Channel 1 of the SSD prototype. . . . . . 229

A.4 Comparison of cosmic ray and laser pulse shapes. . . . . . . . . . 231

A.5 Setup at Brookhaven for the beam test of the SSD prototype. . . . . 232

B.1 Azimuthal angle $(\phi)$ resolution for $J / \psi \rightarrow e^{+} e^{-}$events. . . . . . . . 235

B.2 Distributions of $\theta_{2}^{\text {meas }}-\theta_{2}^{\text {pred }}$ for the SF the CCAL. . . . . . 238

B.3 Schematic showing the geometry of particle detection. . . . . . . 239

B.4 The distribution of the standard deviation $\sigma_{f}$ of $f=\theta_{2}^{\text {meas }}-\theta_{2}^{\text {pred }} \cdot \quad 241$

B.5 Two estimates of the dependence of the errors $\sigma\left(\theta^{\text {meas }}\right)$ on $\theta^{\text {meas }} \ldots . .242$

B.6 Average energy resolution for electrons from $J / \psi \rightarrow e^{+} e^{-}$events. . . . 243

B.7 Number of reconstructed $\pi^{0}$ s. . . . . . . . . . . . . . . . . . 244

B.8 Variation of the average CCAL channel gain constant. . . . . . . . 247

C.1 Schematic of the reaction $\bar{p} p \rightarrow \chi_{2} \rightarrow \gamma \gamma$. . . . . . . . . . . 250

D.1 Gaussian distribution for $x$ and the distribution for $1 / x$. . . . . . 257

D.2 Result of fits to $10,000 \mathrm{MC}$ experiments for $\Gamma\left(\eta_{c}\right)$. . . . . . . 261 


\section{List of Tables}

1.1 The Three Generations of Leptons and Quarks. . . . . . . . . . . . 2

2.1 First-order partial width predictions for $c \bar{c} . \ldots \ldots \ldots$

4.1 Masses of charmonium states and the necessary $\bar{p}$ beam momenta. $\quad 38$

4.2 Characteristics of the 20 CCAL rings. . . . . . . . . 63

5.1 Charged MLU inputs and outputs. . . . . . . . . . . 81

5.2 Super-blocks and their constituents. . . . . . . . . . 86

5.3 Weights for each input to the Level II summers. . . . . . . . . . . 89

5.4 Neutral MLU inputs and outputs. . . . . . . . . . . . 91

5.5 Master MLU inputs and outputs. . . . . . . . . . . . . 95

5.6 Gatemaster Trigger Inputs: priority list . . . . . . . . . . . . 99

5.7 PRUDE trigger priorities. . . . . . . . . . . . . 101

6.1 Parameters for correction of initial position and energy estimates. . . 113

6.2 Estimated thicknesses (in radiation lengths) of the E835 inner detectors.117

xix 
6.3 Fraction of $\gamma$ candidates which appear as "conversions". . . . . . . 122

$7.1 \pi^{0} \pi^{0}\left(\pi^{0} \gamma\right)$ events with $N_{\text {on-time }}$ having an in-time TDC signal. $\quad . .129$

7.2 Efficiencies of various $\chi^{2}$ probability cuts for $J / \psi \rightarrow e^{+} e^{-}$events. . . . 134

7.3 Efficiencies of $\Delta_{R}(\theta)$ and $\Delta_{R}(\phi)$ cuts. (expressed in \%) . . . . 135

7.4 Efficiencies of $\Delta_{R}(\theta)$ cut at 0.020 and $\Delta_{R}(\phi)$ at $0.027 \ldots \ldots 136$

8.1 Results of fitting the background to data for $\left|\cos \theta^{*}\right| \leq 0.2 \ldots \ldots 2$

8.2 Values of $\eta_{c}$ resonance parameters. . . . . . . . . . . 166

8.3 Experimental results for the mass of $\eta_{c} \ldots \ldots . \ldots . \ldots$. . . . . 170

8.4 Experimental results for $\Gamma\left(\eta_{c}\right) \ldots \ldots \ldots \ldots \ldots$

8.5 Experimental results for $\Gamma\left(\eta_{c} \rightarrow \gamma \gamma\right) . \ldots \ldots \ldots . \ldots \ldots 77$

8.6 Summary of experimental results for $\eta_{c}$ from L3. . . . . . . . . . . 179

8.7 Experimental results from E760 and E835 for $\chi_{2}$. . . . . . . 181

8.8 Experimental results for $\Gamma\left(\chi_{2} \rightarrow \gamma \gamma\right)$. . . . . . . . . . . 184

8.9 Summary of results presented in this dissertation. . . . . . . . . 199

8.10 Theoretical predictions for $\gamma \gamma$ and $g g$ partial widths of $\eta_{c}$ and $\eta_{c^{*}}^{\prime} \quad$. . 200

8.11 Theoretical predictions for $\gamma \gamma$ and $g g$ partial widths of $\chi_{0}$ and $\chi_{2}$. $\quad .201$

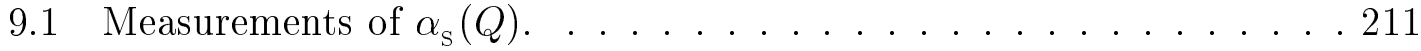

A.1 Results for BNL test of the SSD . . . . . . . . . . . 233

B.1 The effect of target extent and position resolution on $\theta$ resolution. . . 239 
B.2 Results of the schematic calculation of "effective" CCAL resolutions. 241

D.1 Results of fits to $\eta_{c}^{\prime}$ data. . . . . . . . . . . . . 262

E.1 $\gamma \gamma$ candidate events, for $\left|\cos \theta^{*}\right| \leq 0.20$. . . . . . . . . . 265

E.2 $\gamma \gamma$ candidate events, for $\left|\cos \theta^{*}\right| \leq 0.40$. . . . . . . . . 271 


\section{Chapter 1}

\section{Introduction}

Elementary particle physics is the field of physics which involves the study of the fundamental constituents of matter, and the interactions which govern their behavior. The fundamental particles interact by means of four fundamental forces: electromagnetic, weak, strong and gravitational. Of these interactions, three are now encompassed in the Standard Model.

The Standard Model has two sectors: electroweak and strong. The Electroweak sector unifies the electromagnetic and weak interactions. The strong interaction has

its somewhat distinct place in terms of Quantum Chromodynamics (QCD). The fundamental particles of the Standard Model are quarks, leptons and gauge bosons. Quarks participate in all three interactions, while the charged leptons participate in all but the strong interaction. Neutrinos, the neutral leptons, participate only in the weak interaction.

These interactions (with the exception of gravity) are described in terms of quan- 
Table 1.1: The Three Generations of Leptons and Quarks. The masses for quarks are the so-called 'constituent quark' masses. [1]

\begin{tabular}{|c||ccc|lll|}
\hline \multicolumn{1}{|c||}{ Generation } & \multicolumn{2}{c|}{ Quarks } & \multicolumn{3}{c|}{ Leptons } \\
\cline { 2 - 7 } & \multicolumn{3}{|c|}{ Charge } & Mass & \multicolumn{3}{c|}{ Charge } & Mass \\
\hline \hline I & $u$ & $+2 / 3$ & $\sim 300 \mathrm{MeV}$ & $e^{-}$ & -1 & $0.511 \mathrm{MeV}$ \\
& $d$ & $-1 / 3$ & $\sim 300 \mathrm{MeV}$ & $\nu_{e}$ & 0 & $\leq 15 \mathrm{eV}$ \\
\hline II & $c$ & $+2 / 3$ & $\sim 1500 \mathrm{MeV}$ & $\mu^{-}$ & -1 & $105.66 \mathrm{MeV}$ \\
& $s$ & $-1 / 3$ & $\sim 500 \mathrm{MeV}$ & $\nu_{\mu}$ & 0 & $\leq 170 \mathrm{keV}$ \\
\hline III & $t$ & $+2 / 3$ & $173.8 \mathrm{GeV}$ & $\tau^{-}$ & -1 & $1777.05 \mathrm{MeV}$ \\
& $b$ & $-1 / 3$ & $\sim 4700 \mathrm{MeV}$ & $\nu_{\tau}$ & 0 & $\leq 18.2 \mathrm{MeV}$ \\
\hline
\end{tabular}

tum field theories, i.e. in terms of the exchange of gauge bosons. To be specific, the electromagnetic interaction is described in terms of photon $(\gamma)$ exchange, the weak interaction in terms of the exchange of intermediate vector bosons $\left(W^{ \pm}, Z^{0}\right)$, and the strong interaction in terms of gluon $(g)$ exchange.

Experiment E835 at Fermilab is an experiment dedicated to the study the bound states of charmonium: the system composed of a charm quark and a charm antiquark, bound by the strong interaction. This experiment follows in the tradition of Fermilab Experiment E760, many of whose measurements of charmonium state resonance parameters currently dominate the world averages. [2]

This dissertation is devoted to the study of several charmonium states in Experiment E835. Specifically, we address the charmonium states which decay into two photons: $\eta_{c}, \eta_{c}^{\prime}, \chi_{0}$, and $\chi_{2}$. In Chapter 2 we present a theoretical discussion of charmonium spectroscopy, in terms of the quark model and QCD. Because of the close analogy between QCD and Quantum Electrodynamics (QED), we also present certain relevant facts pertaining to QED. In Chapter 3 we discuss the study 
of charmonium spectroscopy from an experimental point of view.

In Chapters 4 through 6, we discuss in detail the experimental aspects of E835 at Fermilab. In Chapter 4 we describe the formation of charmonium states via antiproton-proton annihilation, as well as the experimental apparatus. In Chapter 5, we present details of the E835 data acquisition system, and in Chapter 6, the experimental identification of photons in the E835 detector.

In Chapter 7, we describe the methods we use to reduce our data sample from the trigger data set down to a final set of $\gamma \gamma$ candidate events. In Chapter 8, we present and discuss the final results for various resonance parameters of the charmonium states we have studied, compare them to those from other experiments and theoretical predictions, and draw conclusions. 


\section{Chapter 2}

\section{Charmonium - a Theoretical Background}

\subsection{The Quark Model and the Origins of QCD}

The quark model as a description of the fundamental structure of hadrons is approximately 35 years old. The existence of quarks was first proposed by M. Gell-Mann [3] and G. Zweig [4] in 1964 to explain the classification of hadrons in terms of the SU(3) symmetry group. In the 'eight-fold way' of Gell-Mann, [5], the SU(3) arrangements of light hadrons were realized. The octets of scalar mesons $\left(J^{P}=0^{-}\right)$and the octet (of $J=1 / 2$ ) and decuplet (of $J=3 / 2$ ) baryons were well established, even though the reason for their relationship was not understood. (See Fig. 2.1) The quark model provided the required insight.

The quark model explained the $\mathrm{SU}(3)$ symmetry of strangeness and isospin by the $\mathrm{SU}(3)$ of three flavors of quarks - the up, down and strange quarks. It explained the meson and baryon octets perfectly, but ran into problems with the baryon decuplet, (see Fig. 2.1) whose corner members had to have three identical quarks ( $d d d$ for 


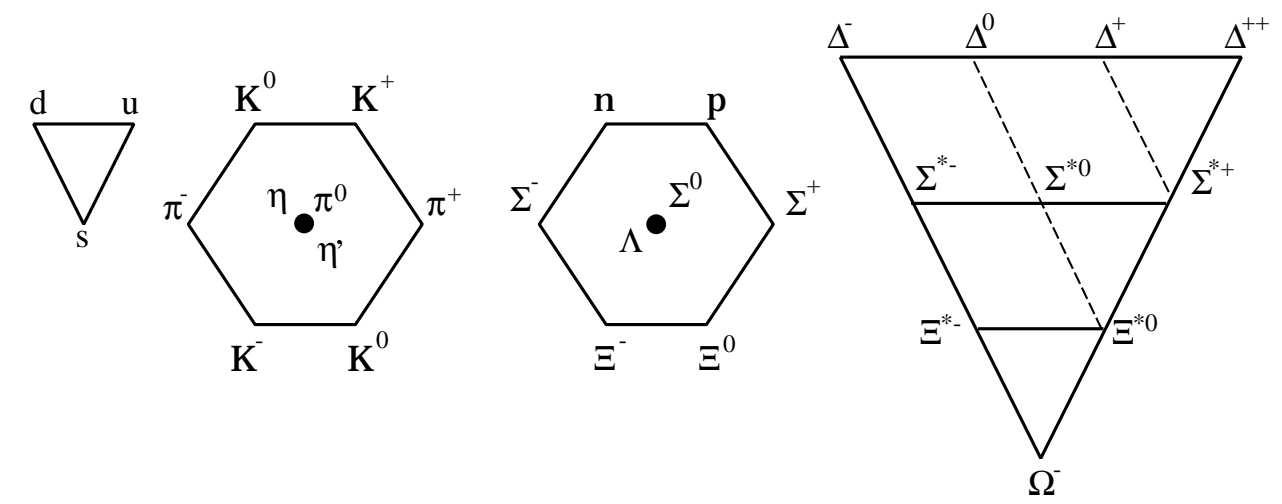

Figure 2.1: $\mathrm{SU}(3)$ classification of light quarks, and of hadrons into a $J^{P}=0^{-}$ meson nonet, a spin-1/2 baryon octet, and a spin-3/2 baryon decuplet.

$\Delta^{-}$, uu for $\Delta^{++}$, and sss for $\Omega^{-}$) in relative s-states. This is, of course, forbidden by the generalized Pauli principle. The problem turned out to provide the most important insight into the quark model - one which transformed the quark model into QCD. The quarks needed another quantum number.

Greenberg called it parastatistics, [6] but it was Gell-Mann's name for it, "color charge", that stuck. [7] Quarks were assigned three colors - red, blue and green, and only color neutral (white) objects were allowed to exist in nature. The interaction between quarks is mediated by gluons $(g)$. Gluons carry both color and anticolor (i.e. there is an octet of gluons, which reflects the SU(3) symmetry of color). Gluons can therefore interact not only with colored quarks, but also with other gluons.

We therefore have quark-antiquark mesons $(q \bar{q}$ - the quark color is balanced by the antiquark's anticolor) and 3-quark baryons ( $q q q$, with one of each color, so that the sum is neutral). Other color-neutral objects are possible, as well: glueballs (2 or 3 gluons, in a color-neutral state), hybrids $(q \bar{q} g)$ or multiquark states $(q \bar{q} \cdot q \bar{q})$. 
These are known collectively as exotic mesons, and some recent experiments have shown strong evidence for the existence of these states. [8, 9]

\section{A charming idea - a fourth quark?}

Even after the introduction of color, the quark model was not entirely satisfying. Some even found it aestheticially unpleasing. The lepton sector, was known to be organized into two doublets $\left(e, \nu_{e}\right)$ and $\left(\mu, \nu_{\mu}\right)$, but for quarks, one had only a doublet of $(u, d)$ quarks, and a singlet $s$ quark. This observation in part led Bjorken

and Glashow [10] to propose a fourth, "up-like" partner to fill the "empty" spot in the strange quark's doublet. Another expectation for a fourth quark, based on more physical considerations, was offered by Glashow, Iliopoulos and Maiani. [11] They proposed a fourth quark to explain some anomalies in the decays of kaons, which were inexplicable in the 3-quark picture.

These predictions were confirmed by experiment in the now-famous "November Revolution" of 1974, which heralded the discovery of $J / \psi$, a narrow resonance with a mass of $\sim 3.1 \mathrm{GeV}$. Very soon after the discovery of $J / \psi, \mathrm{T}$. Appelquist and H. D. Politzer [12] speculated that $J / \psi$ might represent the bound state bound state of a fourth quark (charm) and its antiquark, which was qualitatively similar to positronium, the bound state of a positron and an electron. Further, they postulated the existence of several other positronium-like bound states of the charm quark and antiquark. The expected resemblance to positronium led to the whimsical designation of the expected system as charm-onium. Later investigations at SLAC revealed just such a rich spectrum of hidden charm states. The spectra of both 
positronium and charmonium are displayed in Fig. 2.2.

In the figure, the states are labelled by their quantum numbers $\left(J^{P C}\right)$, the total angular momentum $(\mathrm{J})$, parity $(\mathrm{P})$ and charge conjugation $(\mathrm{C})$. These are related to the total spin S and the orbital angular momentum $\mathrm{L}$ of the system as follows:

$$
J=L+S, \quad P=(-1)^{L+1}, \quad \text { and } C=(-1)^{L+S} .
$$

The states are also designated by the spectroscopic notation $n^{2 S+1} L_{J}$, (where the values of $L=0,1,2 \ldots$ are denoted by the letters $S, P, D \ldots)$ so that the $J^{P C}=0^{-+}$ ground state is written as $1^{1} S_{0}$, and its first radial excitation as $2^{1} S_{0}$. In addition, the states of charmonium have acquired popular names, some based on analogy with states of lighter quarks $\left(\eta_{c}, \eta_{c}^{\prime}\right.$ for ${ }^{1} S_{0}$, and $h_{c}$ for $\left.{ }^{1} P_{1}\right)$, and some based purely on the rights of discovery $\left(J / \psi, \psi^{\prime}\right.$ for ${ }^{3} S_{1}$, and $\chi_{J}$ for $\left.{ }^{3} P_{J}\right)$.

\subsection{Potential Models for the Strong Interaction}

The similarity of positronium and charmonium spectra is quite striking. It was a natural next step after the discovery of charmonium to attempt to model the system as a strongly-interacting analog of positronium. The development of potential models for the strong interaction thus arose from the investigation of the charmonium spectrum. Several of these, including the Cornell potential, [13] Richardson potential, [14] and even some less physically-motivated models taking the form of power law or logarithmic potentials [15] all do a reasonably good job of reproducing the level spacing of the charmonium spectrum. Most notable among these is the 


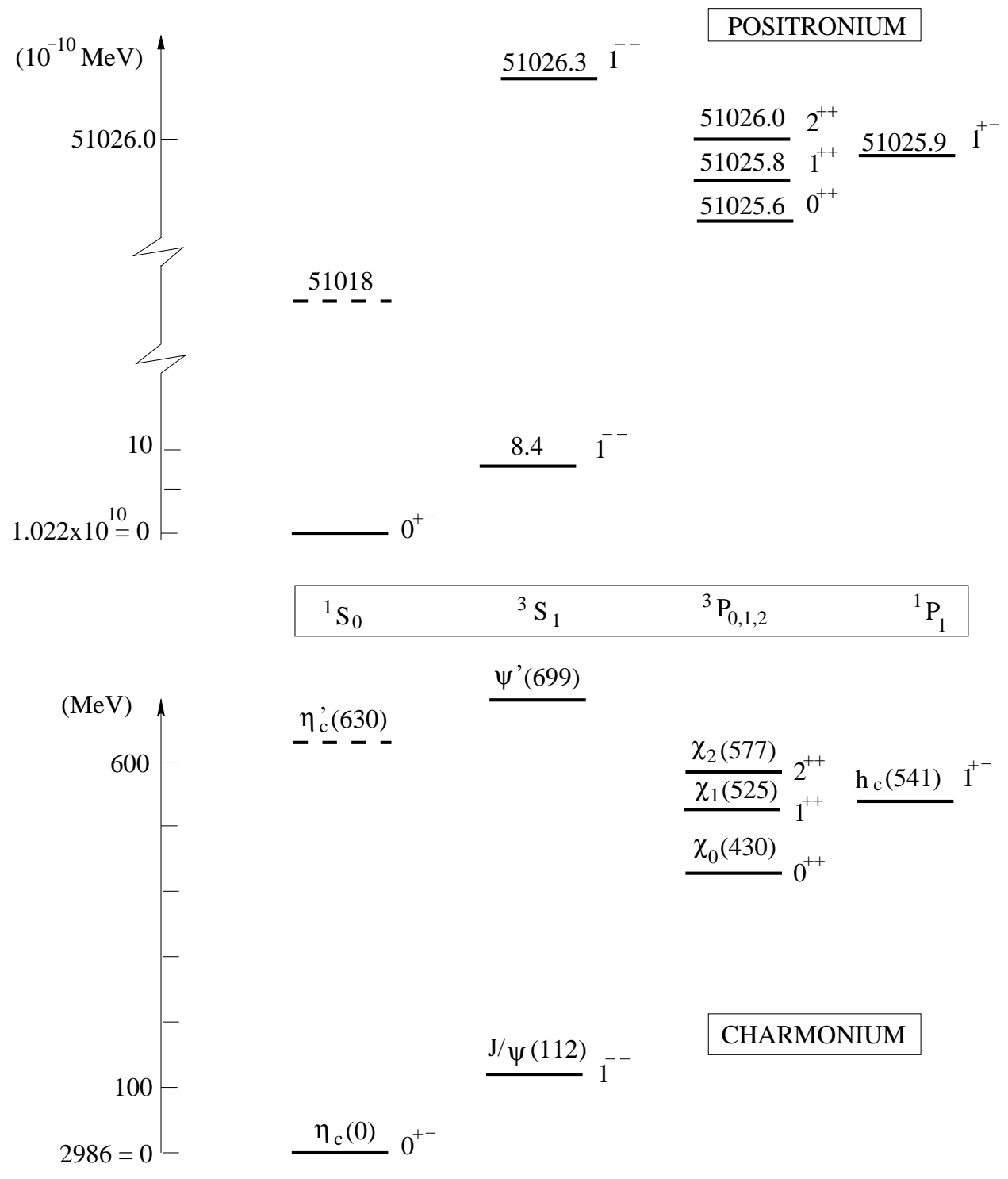

Figure 2.2: A schematic showing the similarities between positronium (top) and charmonium (bottom) spectra. 


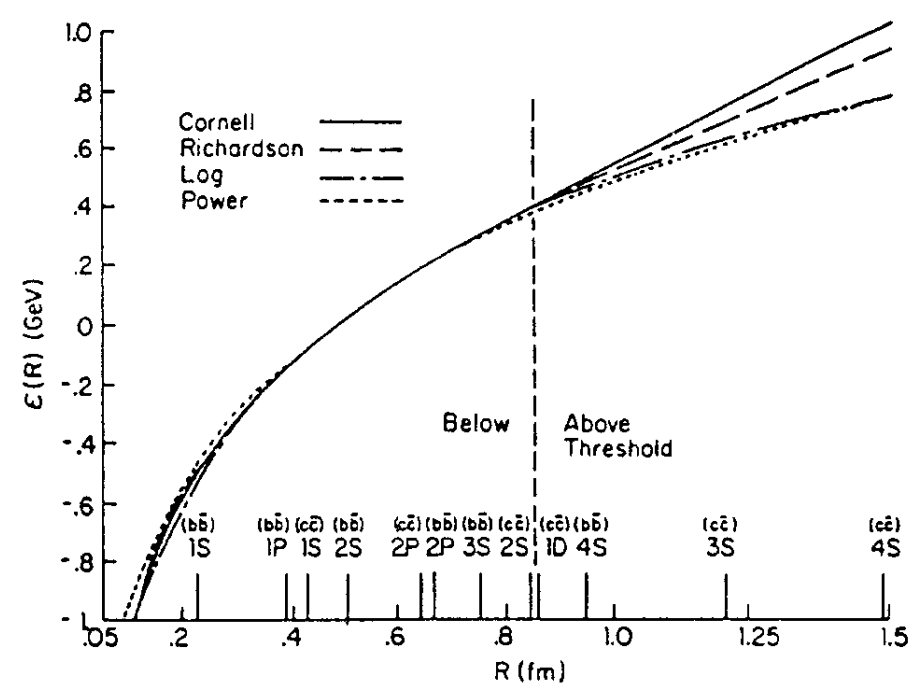

Figure 2.3: Schematic showing various model potentials as a function of quark separation distance $R$. Various $c \bar{c}$ and $b \bar{b}$ levels are indicated by the vertical lines.

Cornell Potential [13],

$$
V(r)=-\frac{4}{3} \frac{\alpha_{\mathrm{S}}}{r}+k r
$$

The first term in the potential should be immediately recognized as Coulombic in nature, analogous to the QED potential. It corresponds to the exchange of a single massless gluon. $\alpha_{\mathrm{S}}$ is the strong coupling constant, and plays the same role in QCD as $\alpha_{\mathrm{EM}}$ does in QED. The second term is the so-called "confinement" term, and reflects the fact that we observe no free quarks in nature. It is often ascribed to the exchange of multiple gluons, and thought of as a "flux tube", or a "string".

For short distances $r$, the Coulombic term is dominant. The smaller the "size" of a meson, the more Coulomb-like is the interaction between its constituents. Thus the low-lying states of charmonium resemble those of positronium, while the higher states may very well reflect significant perturbations due to the confinement poten- 
tial.

The spin dependence of the quark-antiquark interactions is important for the description of structure in the charmonium spectrum. These interactions depend on the Lorentz nature (scalar or vector exchange) of the strong interaction. The Coulomb-like part of the potential is believed to be dominantly vector, since it is presumed to arise from the exchange of a single vector gluon. The confinement term can contain either vector or scalar contributions, since it corresponds to multiplegluon exchange.

Fine structure arises from spin-orbit $(\vec{L} \cdot \vec{S})$ interactions - it can include both vector and scalar contributions. The fine structure in charmonium corresponds to the splitting among the ${ }^{3} P_{J}$ levels, for example. The hyperfine structure of the charmonium system arises from spin-spin $\left(\vec{S}_{1} \cdot \vec{S}_{2}\right)$ interactions between the quarks. The hyperfine splitting for $L=0$ corresponds to the mass difference between the $\eta_{c}$ and $J / \psi$, and between the $\eta_{c}^{\prime}$ and $\psi^{\prime}$.

Potential models which drew on analogies to positronium are quite successful in describing the gross features of the charmonium spectrum. The early theoretical calculations of decay rates of charmonium states were also made by direct analogy with those for positronium. In the next two sections, we introduce briefly the quantum theories of the interactions which give rise to positronium and charmonium, and present some lowest-order calculations of charmonium decay rates, by analogy with similar calculations for positronium. 

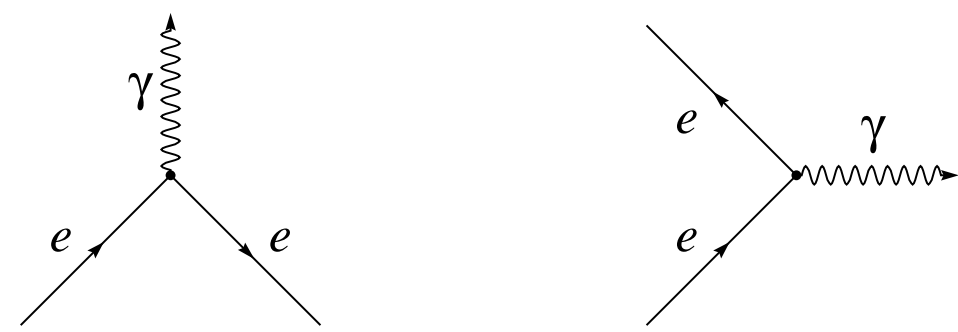

Figure 2.4: Feynman diagrams illustrating the basic QED vertex: (left) $e^{-}$emitting a photon (right) $e^{-}$and $e^{+}$annihilating into a photon.

\subsection{Quantum Electrodynamics and Positronium}

Quantum Electrodynamics (QED) is the quantum theory which describes the interaction between electrically charged particles. Electromagnetic interactions may be expressed in terms of the exchange of a photon or photons between the particles in question. The theory of QED reached maturity in the 1940's and 1950's, on the basis of work by Feynman, Schwinger and Tomonaga [16], for which the three jointly received the 1965 Nobel prize.

The basic QED vertex is represented by the Feynman diagrams shown in Fig. 2.4. Time in the diagrams flows from left to right: fermions have arrows which point to the right, while antifermions have arrows pointing to the left. The diagram on the left shows a fermion (here, an electron) entering the diagram, emitting a photon, and leaving. The diagram on the right shows an electron and positron pair annihilating, producing a single photon.

These diagrams illustrate the basic vertex only; neither diagram is allowed kinematically as drawn. In order to emit a photon as in the diagram on the left in 

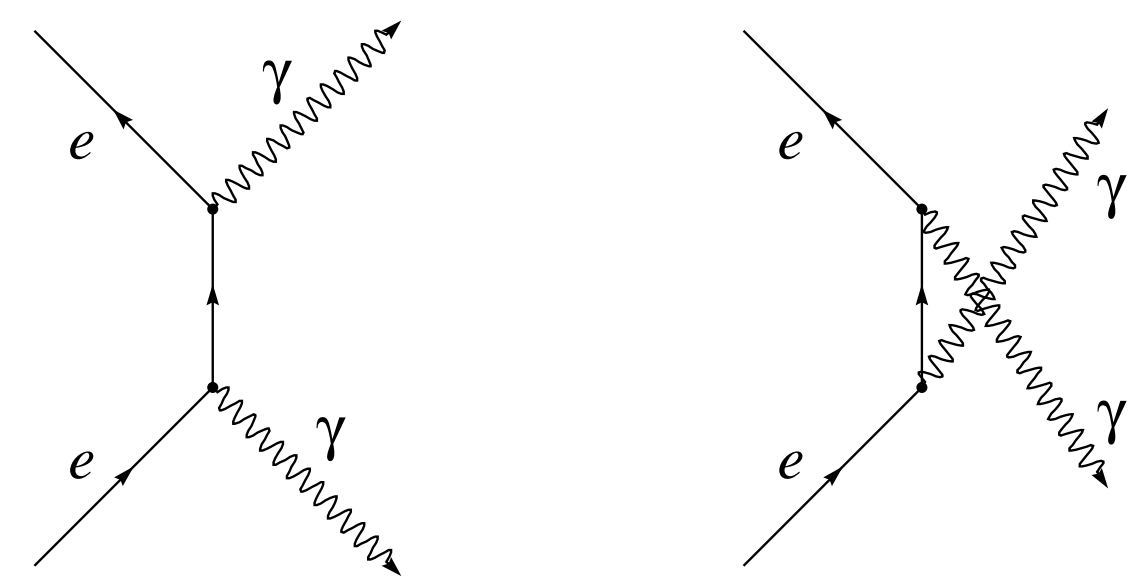

Figure 2.5: The two contributing Feynman diagrams for the 2-photon annihilation process $e^{+} e^{-} \rightarrow \gamma \gamma$

Figure 2.4, the electron must interact with an external electric field, or another charged object. The annihilation diagram on the right is also not possible as drawn. An isolated $e^{+} e^{-}$pair cannot annihilate into a single real photon and conserve fourmomentum. However, the pair can annihilate into two photons (see Figure 2.5). The calculation for this cross section is useful for our discussion, so we outline it here.

Using the rules for Feynman diagrams, we may calculate the cross section for this reaction. The two diagrams in Fig. 2.5 contribute

$$
g_{e}^{2} \bar{v}_{2}\left(\not k_{4} \frac{\left(\not p_{1}-\not p_{3}\right)+m c}{\left(p_{1}-p_{3}\right)^{2}-m^{2} c^{2}} \not k_{3}+\not k_{3} \frac{\left(\not p_{1}-\not p_{4}\right)+m c}{\left(p_{1}-p_{4}\right)^{2}-m^{2} c^{2}} \not k_{4}\right) u_{1}
$$

to the matrix element $\mathcal{M}$, using the notation of Bjorken and Drell. [17] Using the Dirac equation, $\left(\not p_{1}-m c\right) u_{1}=0$, and the rules for permuation of Dirac matrices, 
this simplifies to

$$
\mathcal{M}=-g_{e}^{2} \bar{v}_{2}\left(\frac{\not k_{4} \not p_{3} \not k_{3}}{2 p_{1} p_{3}}+\frac{\not \epsilon_{3} \not p_{4} \not k_{4}}{2 p_{1} p_{4}}\right) u_{1}
$$

in the reference frame in which positron has energy $\mathrm{E}$ and the electron is at rest (This is used for simplicity of calculation; the result is in fact frame independent) and in the Coulomb or transverse gauge (so that $\left(\vec{p}_{1} \cdot \epsilon_{3}\right)=\left(\vec{p}_{1} \cdot \epsilon_{4}\right)=0$ ).

We can then calculate the differential cross section by inserting this expression into a general formula for a two-body process $1+2 \rightarrow 3+4$ :

$$
d \sigma=\int \frac{d^{3} p_{3}}{2 p_{3}} \int \frac{d^{3} p_{4}}{2 p_{4}}(2 \pi)^{4} \delta\left(p_{3}+p_{4}-p_{1}-p_{2}\right) \frac{1}{v_{12}} \frac{m^{2}}{E_{1} E_{2}}|\mathcal{M}|^{2}
$$

For unpolarized initial and final states, we need to average over initial spins and sum over final polarizations. Thus $|\mathcal{M}|^{2}$ is replaced by $(1 / 4) \sum_{f}|\mathcal{M}|^{2}$ :

$$
\begin{aligned}
& d \sigma=\frac{e^{4}}{16 \pi^{2}} \frac{m}{E \beta} \int \frac{d^{3} p_{3}}{2 p_{3}} \int \frac{d^{3} p_{4}}{2 p_{4}} \delta\left(p_{3}+p_{4}-p_{1}-p_{2}\right) \\
& \times \operatorname{Tr} \frac{\not p_{1}-m}{2 m}\left(\frac{\not k_{4} \not p_{3} \not k_{3}}{2 p_{1} p_{3}}+\frac{\not k_{3} \not p_{4} \not k_{4}}{2 p_{1} p_{4}}\right) \frac{\not p_{2}-m}{2 m}\left(\frac{\not k_{3} \not p_{3} \not k_{4}}{2 p_{1} p_{3}}+\frac{\not k_{4} \not p_{4} \not k_{3}}{2 p_{1} p_{4}}\right)(2.6)
\end{aligned}
$$

Using the rules for evaluation of the traces, [17] and performing the phase space integrals to find the total cross section $\sigma$, we obtain, in the non-relativistic limit,

$$
\sigma\left(e^{+} e^{-} \rightarrow \gamma \gamma\right)=\frac{4 \pi}{\beta}\left(\frac{\alpha_{\mathrm{EM}}}{m_{e}}\right)^{2} .
$$

\subsubsection{Application of QED to positronium decays}

The ground state of positronium is the $1^{1} S_{0}$ state. Since there are no fermions lighter than electrons, positronium can only decay by annihilation into photons. No positronium state can decay into a single photon, because that would violate fourmomentum conservation. The ${ }^{1} S_{0}$ state therefore decays into two photons. This 
decay is forbidden to the ${ }^{3} S_{1}$ state by Yang's theorem, [19] so the ${ }^{3} S_{1}$ state can only decay into three photons. We can use the calculation of $\sigma\left(e^{+} e^{-} \rightarrow \gamma \gamma\right)$ to calculate the two-photon decay rate of the positronium state.

The cross section result in Eq. 2.7 can be converted to a width for the annihilation of ${ }^{1} S_{0}$ positronium by multiplying it by the probability that the electron and positron are at the origin $\left(|\psi(0)|^{2}\right.$, the square of the wave function at the origin) and by $\beta$, where $\beta$ is the velocity of the electron or positron in the center of mass frame. We can thus express the width for annihilation of the singlet $\left({ }^{1} S_{0}\right)$ state to lowest order as:

$$
\Gamma\left({ }^{1} S_{0} \rightarrow \gamma \gamma\right)=\frac{4 \pi}{\beta}\left(\frac{\alpha_{\mathrm{EM}}}{m_{e}}\right)^{2} \times \beta|\psi(0)|^{2}=\frac{4 \pi \alpha_{\mathrm{EM}}^{2}}{m_{e}^{2}}|\psi(0)|^{2}
$$

For hydrogen, $|\psi(0)|^{2}$ is well known from elementary quantum mechanics. The positronium 1S wave function is identical to that of hydrogen, except for a difference in length scale. The Bohr radius of the hydrogen atom, which is defined as the mean distance of the electron from the proton, is given by $a=1 /\left(\alpha_{\mathrm{EM}} m_{e}\right)$. In the case of positronium, $m_{e}$ must be replaced by the reduced mass $\mu$, where $\mu=$ $\left(m_{e}\right)\left(m_{e}\right) /\left(m_{e}+m_{e}\right)=(1 / 2) m_{e}$. Thus, the Bohr radius of positronium has the value $a_{\text {pos }}=1 /\left(\alpha_{\mathrm{EM}} \mu\right)=2 /\left(\alpha_{\mathrm{EM}} m_{e}\right)$, or twice that of hydrogen.

So, the positronium $1 \mathrm{~S}$ wave function at the origin is equivalent to that of hydrogen $\left(\psi(0)=\sqrt{\left(8 \pi / a^{3}\right)}\right)$, but with the replacement of the Bohr radius $a$ with $2 a:$

$$
|\psi(0)|^{2}=\frac{1}{\pi a^{3}}=\frac{1}{\pi}\left(\frac{e^{2} m_{e}}{8 \pi}\right)^{3}
$$


Therefore,

$$
\Gamma\left({ }^{1} S_{0} \rightarrow \gamma \gamma\right)=4 \pi\left(\frac{\alpha_{\mathrm{EM}}}{m_{e}}\right)^{2} \frac{m_{e}^{3} e^{6}}{8^{3} \pi^{3}}=\frac{\alpha_{\mathrm{EM}}{ }^{2}}{2^{8}} \frac{m_{e} e^{6}}{\pi^{2} \hbar^{4}}=\frac{1}{2} \alpha_{\mathrm{EM}}{ }^{5} \pi m_{e} .
$$

The lifetime of the state, $\tau=\hbar / \Gamma$, is

$$
\tau=1.2449 \times 10^{-10} \mathrm{sec} .
$$

First and second order QED radiative corrections give: [20]

$$
\Gamma\left({ }^{1} S_{0} \rightarrow \gamma \gamma\right)=\frac{\alpha_{\mathrm{EM}}{ }^{5} \pi m_{e} c^{2}}{2 \hbar}\left(1-\frac{\alpha_{\mathrm{EM}}}{\pi}\left(5-\frac{\pi^{2}}{4}\right)-2 \alpha_{\mathrm{EM}}{ }^{2} \ln \left(\alpha_{\mathrm{EM}}\right)\right)
$$

which modifies the lifetime prediction to:

$$
\tau=1.2516 \times 10^{-10} \mathrm{sec} .
$$

(Notice that the first order radiative correction, $\sim 0.8 \alpha_{\mathrm{EM}}$, amounts to only $\approx 0.6 \%$, because of the small value of $\alpha_{\mathrm{EM}}=1 / 137$.)

Experimentally, the lifetime of the ${ }^{1} S_{0}$ ground state of positronium is [21]:

$$
\tau=1.2515 \pm 0.0003 \times 10^{-10} \mathrm{sec},
$$

which is in very good agreement with the QED prediction.

\subsection{Quantum Chromodynamics}

Quantum Chromodynamics (QCD) is the theory which has been developed over the past three decades to describe the strong interaction as a quantum field theory. 

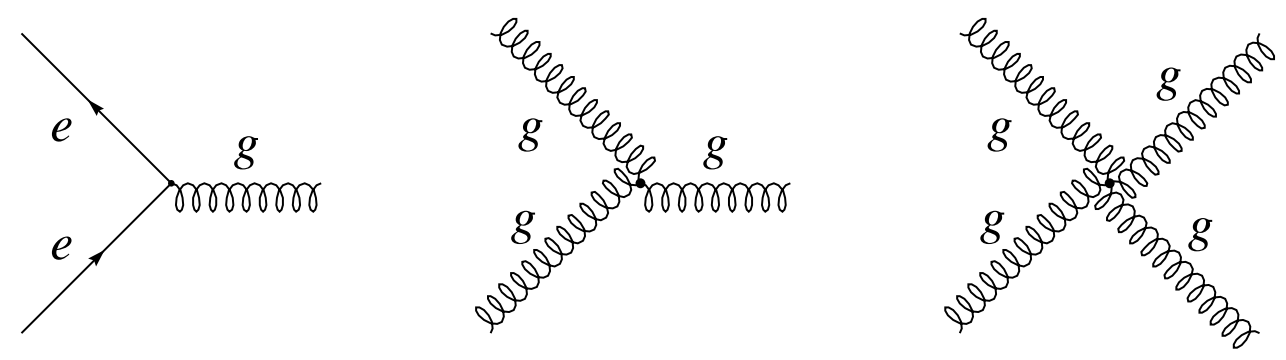

Figure 2.6: The three basic QCD vertices.

It is not nearly as transparent as QED, partly because it is mathematically more intricate. It is nonetheless quite successful in describing the basic hadronic structures we observe.

Since the mediator of QCD, the gluon, can interact not only with quarks, but with other gluons, there are three basic vertices in QCD (c.f. QED's single elementary vertex) and these are shown in Fig. 2.6. Ultimately, this gluon self-interaction manifests itself in the two phenomena we know as confinement and asymptotic freedom.

\subsection{Application of QED and QCD to charmonium decays}

We discuss two types of processes involving charmonium in this dissertation. Each of the states investigated in this work couples to two photons, and similarly to two gluons. We can arrive at estimates for the partial widths of $c \bar{c} \rightarrow \gamma \gamma$ and $c \bar{c} \rightarrow g g$, using the result of the calculation of the $e^{+} e^{-} \rightarrow \gamma \gamma$ cross section presented in Sec. 2.3.

For instance, by replacing the coupling constant $\alpha_{\mathrm{EM}}$ in Equation 2.8 by the 
appropriate value for the charm quark, $\left(\frac{2}{3}\right)^{2} \times \alpha_{\mathrm{EM}}=\frac{4}{9} \alpha_{\mathrm{EM}}$, and $m_{e}$ by $m_{c}$, and multiplying by 3 (for the 3 possible color combinations allowed for the charmonium system) we arrive at a lowest order estimate of $\Gamma\left(c \bar{c}\left({ }^{1} S_{0}\right) \rightarrow \gamma \gamma\right)$ :

$$
\Gamma\left(c \bar{c}\left({ }^{1} S_{0}\right) \rightarrow \gamma \gamma\right)=\frac{64 \pi \alpha_{\mathrm{EM}}{ }^{2}}{27 m_{c}^{2}}\left|\psi_{c \bar{c}}^{S}(0)\right|^{2} .
$$

Since the strong interaction potential isn't precisely Coulombic, even at very short range, we cannot assume that the charmonium wave function is a Coulomb wave function, like we did in Equation 2.10. Thus we leave the prediction in terms of the $1 \mathrm{~S}$ charmonium wave function at the origin $\psi(0)$.

We can estimate the value of the two-gluon annihilation width of ${ }^{1} S_{0}$ charmonium in a similar manner as we have the $\gamma \gamma$ width. Assuming that one gluon exchange dominates the charm-anticharm interaction, we can substitute into Eq. 2.8 the strong coupling constant $\alpha_{\mathrm{S}}$ for $\alpha_{\mathrm{EM}}$, along with the appropriate combinatoric color factor of $(9 / 8)$, to arrive at the lowest order result for $\Gamma\left(c \bar{c}\left({ }^{1} S_{0}\right) \rightarrow g g\right)$ :

$$
\Gamma\left(c \bar{c}\left({ }^{1} S_{0}\right) \rightarrow g g\right)=\frac{8 \pi}{3} \frac{\alpha_{\mathrm{S}}^{2}}{m_{c}^{2}}\left|\psi_{c \bar{c}}^{S}(0)\right|^{2}
$$

Similar, somewhat more complicated calculations can be made for the $\gamma \gamma$ and $g g$ decay rates of the ${ }^{3} P_{2}$ and ${ }^{3} P_{0}$ states as well.

In the discussion of our results in Chapter 8, we will be interested in comparing the $\gamma \gamma$ partial widths of the ${ }^{1} S_{0}$ charmonium states with the leptonic partial widths of the corresponding ${ }^{3} S_{1}$ states. The calculation of $\Gamma\left({ }^{3} S_{1} \rightarrow e^{+} e^{-}\right)$is also a simple QED calculation, following directly from the calculation of cross section for the process $e^{+} e^{-} \rightarrow \mu^{+} \mu^{-}$. The calculation of the leptonic widths of vector mesons was presented in 1967 by Van Royen and Weisskopf [22]. In terms of the mass of the 
Table 2.1: First-order partial width predictions and the first order radiative corrections. [23] According to Kwong, et al. , the radiative corrections have typical uncertainties of $\pm \alpha_{\mathrm{S}} / \pi$, or approximately $\pm 11 \%$.

\begin{tabular}{|l||l|l|l|}
\hline Partial & Lowest order & First Order & Correction \% \\
Width & Prediction $\Gamma_{0}$ & Correction & $\alpha_{\mathrm{S}}=0.35$ \\
\hline${ }^{1} S_{0} \rightarrow \gamma \gamma$ & $64 \pi \alpha_{\mathrm{EM}}{ }^{2}\left|\psi_{S}(0)\right|^{2} / 27 m_{c}^{2}$ & $1-3.4 \alpha_{\mathrm{S}} / \pi$ & -37.8 \\
${ }^{1} S_{0} \rightarrow g g$ & $8 \pi \alpha_{\mathrm{S}}{ }^{2}\left|\psi_{S}(0)\right|^{2} / 3 m_{c}^{2}$ & $1+4.8 \alpha_{\mathrm{S}} / \pi$ & 53.5 \\
${ }^{3} S_{1} \rightarrow e^{+} e^{-}$ & $64 \alpha_{\mathrm{EM}}{ }^{2}\left|\psi_{S}(0)\right|^{2} / 9 M^{2}$ & $1-5.3 \alpha_{\mathrm{S}} / \pi$ & -59.0 \\
${ }^{3} P_{0} \rightarrow \gamma \gamma$ & $16 \alpha_{\mathrm{EM}}{ }^{2}\left|R_{P}^{\prime}(0)\right|^{2} / 3 m_{c}^{4}$ & $1+0.2 \alpha_{\mathrm{S}} / \pi$ & 2.2 \\
${ }^{3} P_{0} \rightarrow g g$ & $6 \alpha_{\mathrm{S}}{ }^{2}\left|R_{P}^{\prime}(0)\right|^{2} / m_{c}^{4}$ & $1+9.5 \alpha_{\mathrm{S}} / \pi$ & 105.8 \\
${ }^{3} P_{2} \rightarrow \gamma \gamma$ & $64 \alpha_{\mathrm{EM}}{ }^{2}\left|R_{P}^{\prime}(0)\right|^{2} / 45 m_{c}^{4}$ & $1-5.3 \alpha_{\mathrm{S}} / \pi$ & -59.0 \\
${ }^{3} P_{2} \rightarrow g g$ & $8 \alpha_{\mathrm{S}}{ }^{2}\left|R_{P}^{\prime}(0)\right|^{2} / 5 m_{c}^{4}$ & $1-2.2 \alpha_{\mathrm{S}} / \pi$ & -24.5 \\
\hline
\end{tabular}

vector state $M_{V}$, and the wave function at the origin $\psi(0)$, the leptonic decay rate for vector charmonia is given by:

$$
\Gamma\left(c \bar{c}\left({ }^{3} S_{1}\right) \rightarrow l^{+} l^{-}\right)=\frac{64 \pi \alpha_{\mathrm{EM}}}{9 M_{V}^{2}}|\psi(0)|^{2} .
$$

First order QCD radiative corrections have been made to all these partial width predictions, and are summarized in Ref. [23]. Each of the corrected widths has the form $\Gamma=\Gamma_{0} \times\left(1+B \alpha_{\mathrm{S}}\right)$, where $\Gamma_{0}$ is the lowest order partial width prediction. These partial width predictions and their first-order radiative corrections are presented in Table 2.1. We note that because of the largeness of the strong coupling constant $\alpha_{\mathrm{S}},(\approx 0.35$ in the charmonium region $)$ the first order radiative corrections are large $(20 \%-100 \%)$, raising serious questions about their validity.

The formulae in the Table 2.1 are not very useful in making absolute width 
predictions, because of the presence of several unknown quantities. In all cases, $\alpha_{\mathrm{S}}$ and $m_{c}$ appear, as well as the total wavefunctions $\left|\psi_{S}(0)\right|^{2}$ or the radial wave function derivatives $\left|R_{P}^{\prime}(0)\right|^{2}$ at the origin. All of these are unknown, so the best practical use of these width predictions is in terms of their ratios, constructed so as to cancel $m_{c}$ as well as $\left|\psi_{S}(0)\right|^{2}$ or $\left|R_{P}^{\prime}(0)\right|^{2}$. These ratios can then be used to determine values of $\alpha_{\mathrm{S}}\left(m_{c}\right)$ from the experimental data. We will present such an analysis in Chapter 8, using our data taken at $\eta_{c}$ and $\chi_{2}$, and appropriate ratios constructed from the partial widths in the above table. 


\section{Chapter 3}

\section{Experimental Study of Charmonium}

\subsection{Why charmonium?}

One might rightly ask why it is charmonium, and not another quark-antiquark system, which one should study in order to understand the strong interaction. It is a well-known experimental fact that light quark mesons, e.g. the $\phi(s \bar{s})$, or the $\rho(u \bar{u}, d \bar{d})$, are far more prolifically produced than charmonium, regardless of the production mechanism. In general, the cross sections for the production of quarkonia decrease rapidly with their mass. Thus, from the point of view of statistical precision alone, light quark mesons would be the preferred subject of study. However, statistics is not the only criterion which we must consider.

In a light quark meson, the constituent quarks are quite relativistic. It is estimated that $\left(\left\langle v^{2} / c^{2}\right\rangle \simeq 1.0(u \bar{d}), \simeq 0.85(u \bar{s}), \simeq 0.7(s \bar{s})\right)$ As such, these systems are poorly described by a non-relativistic formulation of the strong interaction. 
In addition, it is well-known that the coupling constant of QCD, $\alpha_{\mathrm{S}}(q)$, grows to rather large values $(\sim 0.5)$ at the small momentum scales $(q)$ characteristic of light quark systems. The QCD interaction, which is perturbative at high $q$, becomes dominated by non-perturbative effects at low $q$. Thus, predictions for light quark systems become very unreliable.

A third problem arises because of the near equality of the $u, d$ and $s$ quark masses. Aside from the few mesons below $\sim 1 \mathrm{GeV}$, mesons composed of $(u, d, s)$ quarks and antiquarks are nine times as numerous (as a single flavor $q \bar{q}$ ), they acquire very similar masses, and the large decay widths (typically 100 to $500 \mathrm{MeV}$ ). Their spectra become extremely dense and overlapping. Spectroscopy of such states becomes quite difficult, and often requires computationally-intensive partial wave analyses to make even the simplest identifications. These complications would suggest avoiding the light quark systems.

In contrast, both the relativistic and non-perturbative effects are smaller for charmonium than for light quark mesons; average charm quark velocities are $\left\langle v^{2} / c^{2}\right\rangle \simeq$ 0.25 , and the coupling constant $\alpha_{\mathrm{S}}\left(m_{c}\right) \simeq 0.3$. Production cross sections for charmonium resonances are reasonably large. Further, the charmonium spectrum is characterized by well-spaced states, with narrow widths - so that they are well resolved. Spectroscopy of charmonium is thus clean and can be made very precise.

Precision spectroscopy of such simple two-body systems as charmonium is a good tool with which to examine the physics of the underlying strong interaction, just as the study of the hydrogen atom and positronium have contributed greatly to the understanding of the electromagnetic interaction which binds them. Besides 
providing insight into the QCD interaction, the spectroscopy of the charmonium system may also be expected to shed light on the nature and properties of the charm quark.

\subsection{Discovery of Charmonium}

In 1974, two groups, one at the Brookhaven National Laboratory, and the other at the Stanford Linear Accelerator Center, simultaneously announced the discovery of a resonance with a mass of $\sim 3.1 \mathrm{GeV}$ and a surprisingly narrow width of $\sim 100 \mathrm{keV}$. (See Fig. 3.1) The group at BNL, led by Sam Ting, was studying the production of dilepton pairs in proton-Beryllium collisions; they called the state they observed "J". [24] The SLAC group, led by Burton Richter, was measuring the hadronic cross section in $e^{+} e^{-}$annihilation in the vicinity of $3 \mathrm{GeV}$, they called the state they found " $\psi$ ". [25] Though a number of imaginative interpretations for this state, $J / \psi$, were

offered, [26] it became clear very soon that it had a 'natural' explanation in terms of a new quark and its antiquark partner. The 'charm' quark anticipated by Bjorken, Glashow, Iliopoulos and Maiani had arrived.

Ting and Richter subsequently shared the 1976 Nobel Prize in Physics for this momentous discovery. It is perhaps not hyperbolic to say that the discovery of bound charm brought the quark model from its existence merely as a mathematical tool to account for the classification of hadrons according to $\mathrm{SU}(3)$ symmetry, to its recognition as a legitimate model of hadron structure.

The detailed spectrum of charmonium states with major decays indicated is 

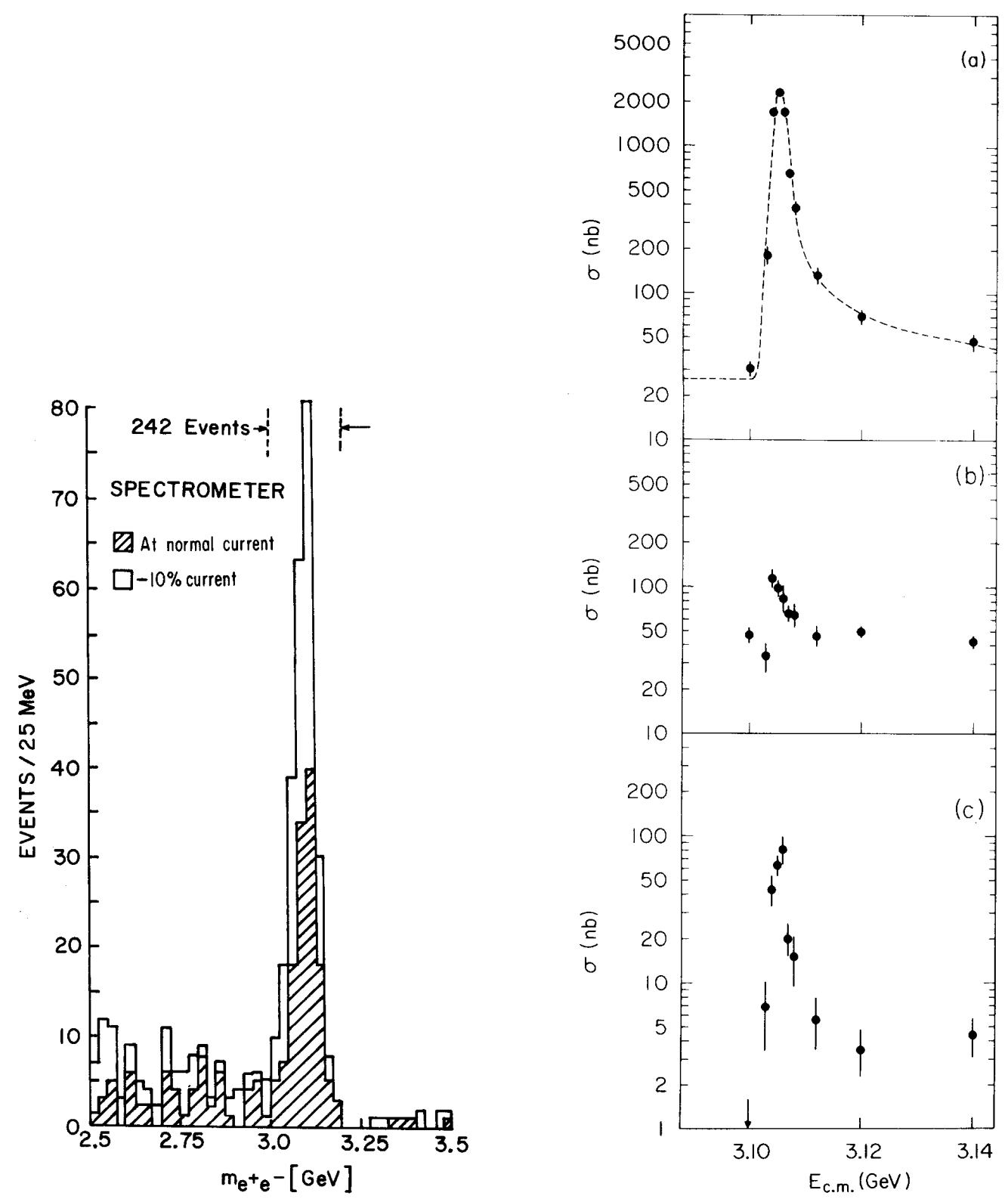

Figure 3.1: Spectra demonstrating the first experimental evidence in 1974 for the resonance known as $J / \psi$, from (left) the BNL experiment [24] and (right) the SLAC experiment. [25] 
shown in Fig. 3.2. Charmonium has been studied primarily by $e^{+} e^{-}$annihilation, and more recently by $\bar{p} p$ annihilation. We describe each of these techniques in this chapter.

\subsection{Charmonium Production in $e^{+} e^{-}$collisions}

Aside from the $J / \psi$ discovery experiment in p-Be collisions at Brookhaven, charmonium was studied almost exclusively at electron-positron colliders. All bound states of charmonium (below the open charm threshold at $\sim 3.7 \mathrm{GeV}$ ) were either observed or claimed to have been observed. Not all were well studied, however, for various reasons.

The best studied of the charmonium states were the $J / \psi$ and $\psi^{\prime}$ - the vector states $\left(J^{P C}=1^{--}\right)$. These are the only states which can be directly formed in the annihilation of $e^{+} e^{-}$, since $e^{+} e^{-}$annihilation is mediated by a virtual photon, whose quantum numbers are $J^{P C}=1^{--}$. (See Fig. 3.3) Because these states were formed directly, their masses and widths could be measured with (good) precision, which depended only upon the precision in the knowledge of the electron/positron beam parameters.

In direct formation, the excitation curve of the vector state under investigation may be measured. This was done by means of a 'scan', in which the $e^{+}$and $e^{-}$ beam energies were stepped in small increments so that the center of mass energy moves across the resonance. This method was used to produce the SLAC data for the $J / \psi$ discovery shown in Fig. 3.1(b). The mass of the resonance is simply given 


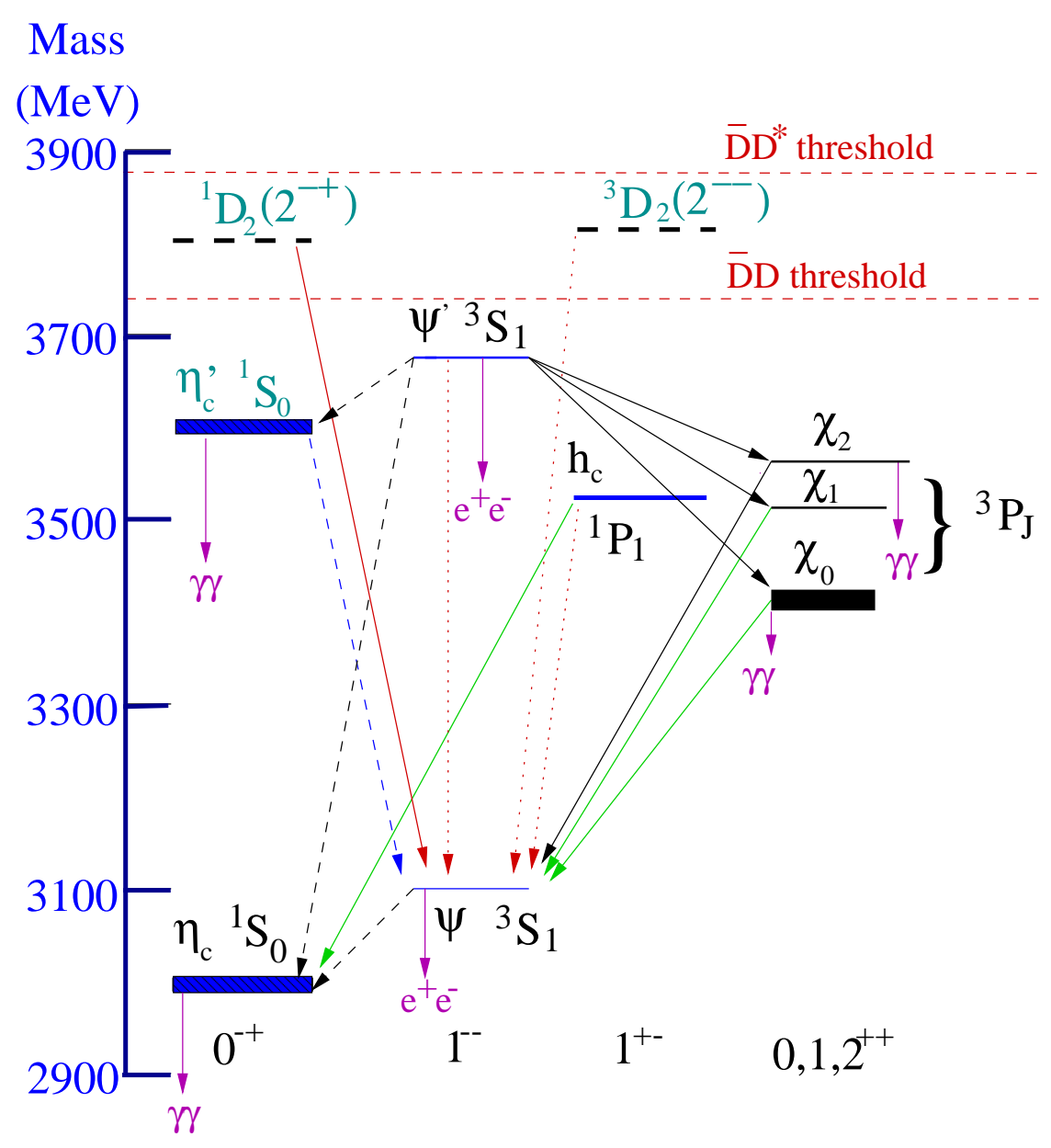

Figure 3.2: The spectrum of charmonium states. Unlabelled solid lines and dashed lines represent E1 and M1 radiative transitions, respectively. Dotted lines represent hadronic transitions. 


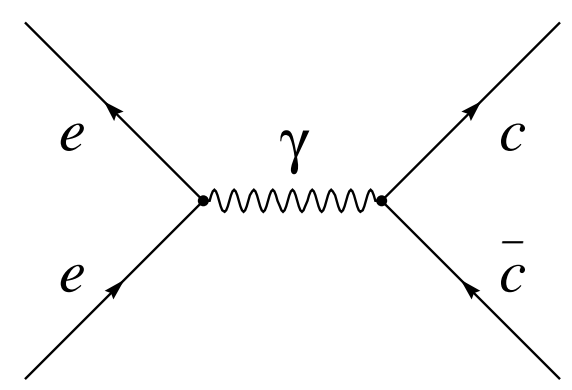

Figure 3.3: Formation of vector charmonium states via $e^{+} e^{-}$annihilation.

by analyzing the measured excitation function.

It was not possible to measure the widths of $J / \psi$ and $\psi^{\prime}$, in such a direct manner. At SLAC, the $e^{+}$and $e^{-}$beam energy spreads and initial state radiation from the $e^{+}$and $e^{-}$beams resulted in a center of mass energy spread of $\sim 2.6 \mathrm{MeV}$. The total widths of $J / \psi$ and $\psi^{\prime}$, however, are an order of magnitude smaller. Thus their total widths had to be determined by measuring the areas under the measured excitation curves for the leptonic and hadronic decays of $J / \psi$ and $\psi^{\prime}$. This required knowledge of detector acceptance and efficiency for the final state particles, and corrections for distortion of the excitation curves due to initial and final state radiation (bremsstrahlung). The resulting precision in the determination of the $J / \psi$ and $\psi^{\prime}$ widths was therefore compromised.

Non-vector states of charmonium $\left(e . g .,{ }^{1} S_{0},{ }^{1} P_{1},{ }^{3} P_{J}\right)$ were only observed as products of radiative decay of $\psi^{\prime}$, and as a result they were produced much less copiously. Since their detection required the observation of several hundred $\mathrm{MeV}$ photons, the determination of their masses and total widths depended upon the 
properties of the photon detectors. The energy resolution of the best of the SLAC photon detectors, the Crystal Ball, ranged from 20 to $30 \mathrm{MeV}$ for the photon energies in question. As a result, although the masses of the ${ }^{3} P_{J}$ states, observed in the E1 decays of $\psi^{\prime}$, were determined to $\sim \pm 1 \mathrm{MeV}$, it was only possible to set rather liberal limits for the widths. The study of singlet-S charmonium states $\left(\eta_{c}\right.$ and $\left.\eta_{c}^{\prime}\right)$ was further hampered by the fact that they could only be observed in the significantly weaker M1 radiative decay of $\psi^{\prime}$ and/or $J / \psi$. As a result, $\eta_{c}$ resonance parameters were poorly determined, and the observation of $\eta_{c}^{\prime}$ was claimed, but was never confirmed. Branching ratios for the few $\eta_{c}$ decays which were measured all had errors $\geq 30 \%$. The production of the singlet-P state $\left(h_{c}\right)$ in the radiative decay of $\psi^{\prime}$ is, of course, forbidden by charge conjugation, and it was not observed.

Recently, the electron-positron collider at Beijing's BEPC has been studying charmonium, but their measurements continue to have the inherent limitations of the $e^{+} e^{-}$annihilation method.

\subsection{Charmonium Production in $\bar{p} p$ collisions}

In 1984, the R704 experiment at CERN pioneeered the technique of studying charmonium resonances by forming them in $\bar{p} p$ annihilation and detecting them via their electromagnetic decays. A hydrogen gas jet target intersected the cooled, circulating antiproton beam stored in one of the ISR storage rings, and the reaction products were identified in a two-arm electromagnetic detector. Several charmonium states

were investigated, but for various reasons the precision of the results was rather 

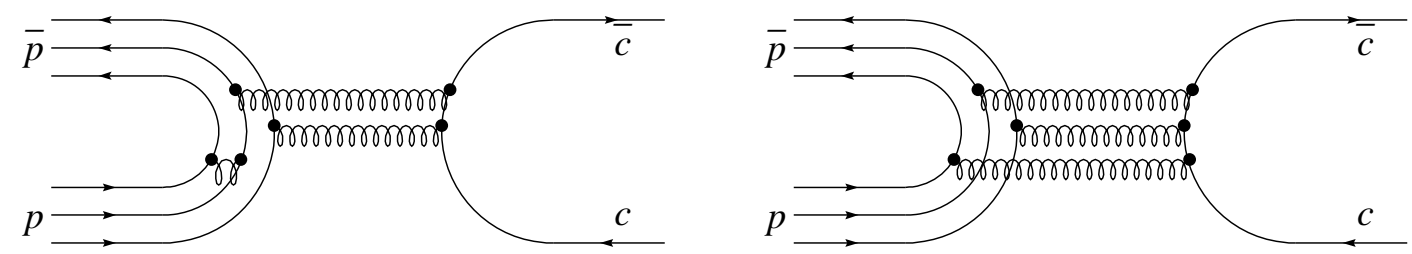

Figure 3.4: Formation of charmonium states via $\bar{p} p$ annihilation.

limited.

Fermilab Experiments E760 (1991-2) and E835 (1996-7) have used the $\bar{p} p$ annihilation technique to study charmonium spectroscopy at the Fermilab Antiproton Accumulator with a much improved beam and detector. The technique has distinct advantages over the production of charmonium states via $e^{+} e^{-}$annihilation.

All charmonium states can be formed directly by $\bar{p} p$ annihilation. The formation of all $J^{P C}$ combinations is possible because the antiproton and proton annihilate into either two gluons $(\mathrm{C}=+1)$ or three gluons $(\mathrm{C}=-1$ ). (See Fig. 3.4) The quantum numbers of the intermediate state may therefore take on any $J^{P C}$, since each of the gluons carries $J^{P C}=1^{--}$, and there may be orbital angular momentum $L$ among them. The fact that all states can be formed directly is a major advantage of this technique over the $e^{+} e^{-}$annihilation technique, because it permits the precision of the accelerator beam to directly bear on the quality of the measurements for all charmonium resonances.

In a $\bar{p} p$ annihilation experiment the excitation function of any charmonium resonance can be directly measured by stepping (or "scanning") the energy of the $\bar{p}$ beam in small increments across the resonance. The determination of the mass 
of any charmonium state is thus directly obtained from the excitation curve, and depends only the precision of the beam energy parameters. The $\bar{p}$ beam energy distribution can be made very small by stochastic cooling, and it can be very well measured in terms of the beam circulation frequency and the orbit length. Further, because $m_{p} \approx 1800 m_{e}$, the distortion of the excitation function caused by initial state radiation is far smaller in $\bar{p} p$ annihilation than in the $e^{+} e^{-}$case, This leads to direct measurements of resonance widths, which require only deconvolution of the small width of the $\bar{p}$ beam energy distribution.

Thus, the $\bar{p} p$ annihilation technique leads to much better precision in mass and width measurements for all charmonium states than was possible in the $e^{+} e^{-}$annihilation experiments. 


\section{Chapter 4}

\section{Fermilab Experiment E835}

In this chapter we describe the operation of Fermilab E835, including the production and accumulation of $\bar{p}$ 's at Fermilab, energy measurements of the $\bar{p}$ beam, the hydrogen gas jet target, and the precision measurements of luminosity, which are necessary for the study of the narrow states of charmonium. We also discuss the E835 detector system, with special emphasis on the primary detector, the Central Calorimeter.

\section{1 $\bar{p}$ Production, Cooling and Accumulation}

Antiprotons are produced at Fermilab by the following sequence of operations. [27] Negatively-charged hydrogen ions $\left(H^{-}\right)$are accelerated in a Cockroft-Walton accelerator to an energy of $750 \mathrm{keV}$. This beam is then accelerated by a linear accelerator to $200 \mathrm{MeV}$. The $H^{-}$ions are then stripped of their electrons by a carbon foil through which the beam passes, and the resulting beam of $200 \mathrm{MeV}$ protons is injected into 


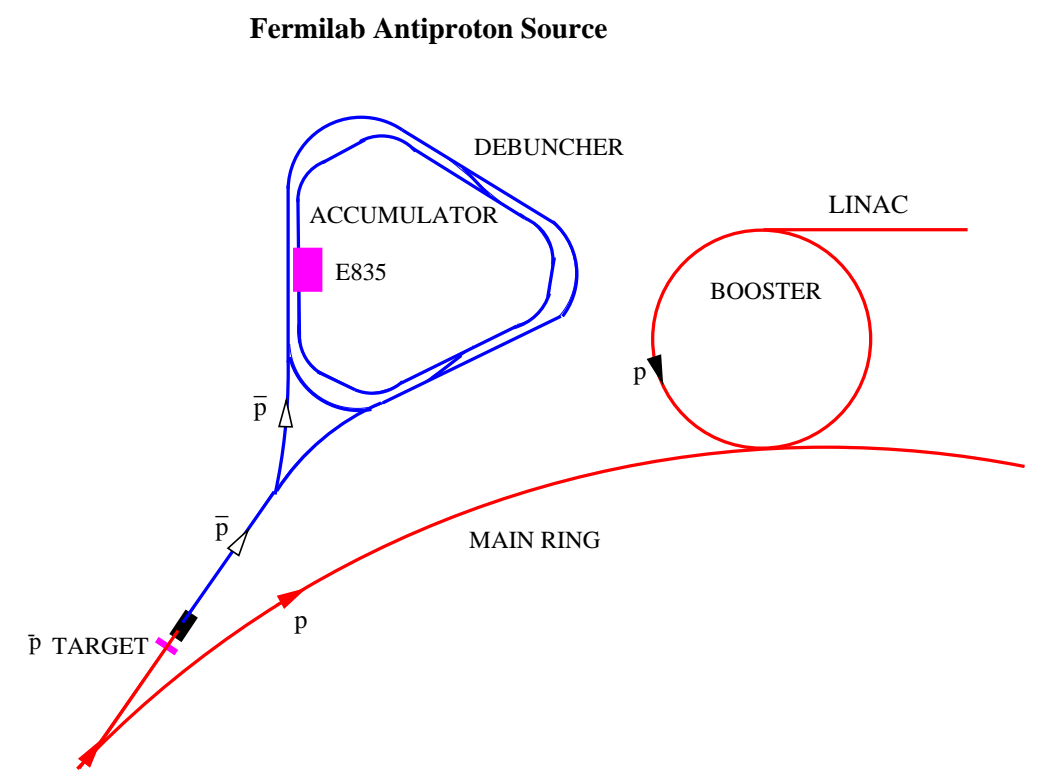

Figure 4.1: Schematic of the Fermilab Antiproton Accumulator and other Fermilab accelerator components. The target region is shown in detail in Fig. 4.2.

the Booster synchrotron, which accelerates the beam to $8 \mathrm{GeV}$. This cycle is repeated every two seconds, each time producing a pulse of approximately $2 \times 10^{12}$ protons, which are said to constitute a "batch". The batches have a substructure of $53 \mathrm{MHz}$ bunches. Each of the 82 bunches in a batch is $\sim 1 \mathrm{~ns}$ in length, separated from each other in time by $1 / 53 \mathrm{MHz} \approx 19 \mathrm{~ns}$.

Each batch of $8 \mathrm{GeV}$ protons is then injected from the Booster (See Fig. 4.1) into the synchrotron which is known as the Main Ring, where it is accelerated to 120 $\mathrm{GeV}$. Antiproton production is then accomplished by extracting the proton batch from the Main Ring and directing it onto a 6-cm long tungsten target. Antiprotons and other negatively charged particles produced in these collisions are collected by a lithium lens.(See Fig. 4.2) 


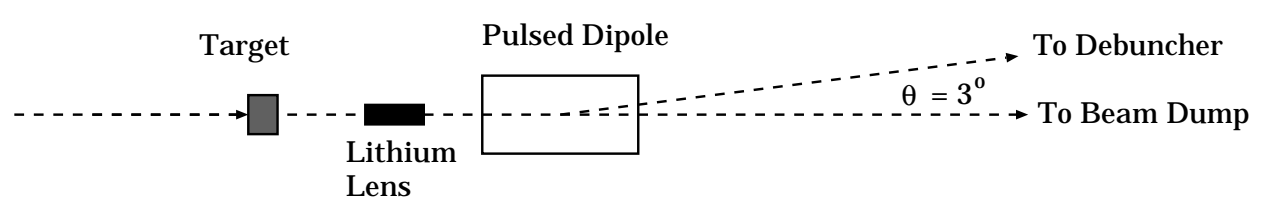

Figure 4.2: Schematic of the antiproton production target area.

The lithium lens is a $15 \mathrm{~cm}$ long cylinder of $2 \mathrm{~cm}$ diameter, which typically carries an electric current of $\sim 0.6 \times 10^{6}$ amps along its axis. This current gives rise to an azimuthal magnetic field of $\sim 750 \mathrm{~T} / \mathrm{m}$ which acts to focus part of the divergent spray of particles into an unseparated beam. This beam is then passed through a pulsed dipole magnet which selects particles with momenta of $8.9 \pm 0.36 \mathrm{GeV} / \mathrm{c}$ $(\Delta p / p= \pm 4 \%)$, giving them a $3^{\circ}$ deflection in the process and directing them into a beam line, and subsequently into the $\sim 500 \mathrm{~m}$ circumference Debuncher Ring. The yield of antiprotons into the Debuncher Ring is about $1 \times 10^{-5} \bar{p}$ per proton, or $2 \times 10^{7} \bar{p}$ per 2 -second cycle.

For a successful accumulation of antiprotons in the Accumulator Ring, the input momentum spread $\Delta p / p$ of $\pm 4 \%(\approx \pm 0.36 / 8.9)$ in the Debuncher Ring is too large. However, it can be reduced considerably at the cost of the tight bunch structure of the antiprotons, i.e. by "de-bunching" the beam by RF manipulation, as described below. Consider a single antiproton bunch, which has been injected into an RF bucket in the Debuncher, whose RF voltage is large enough to accept its full momentum spread $(\Delta p)$. While the allowed momentum spread is large enough, the spread in time of the bunch $\left(\Delta t_{\text {bunch }} \simeq 1 \mathrm{~ns}\right)$ is much smaller than that of the bucket $\left(\Delta t_{\text {bucket }} \simeq 19 \mathrm{~ns}\right)$. When a bunch is injected into such an RF bucket, it 

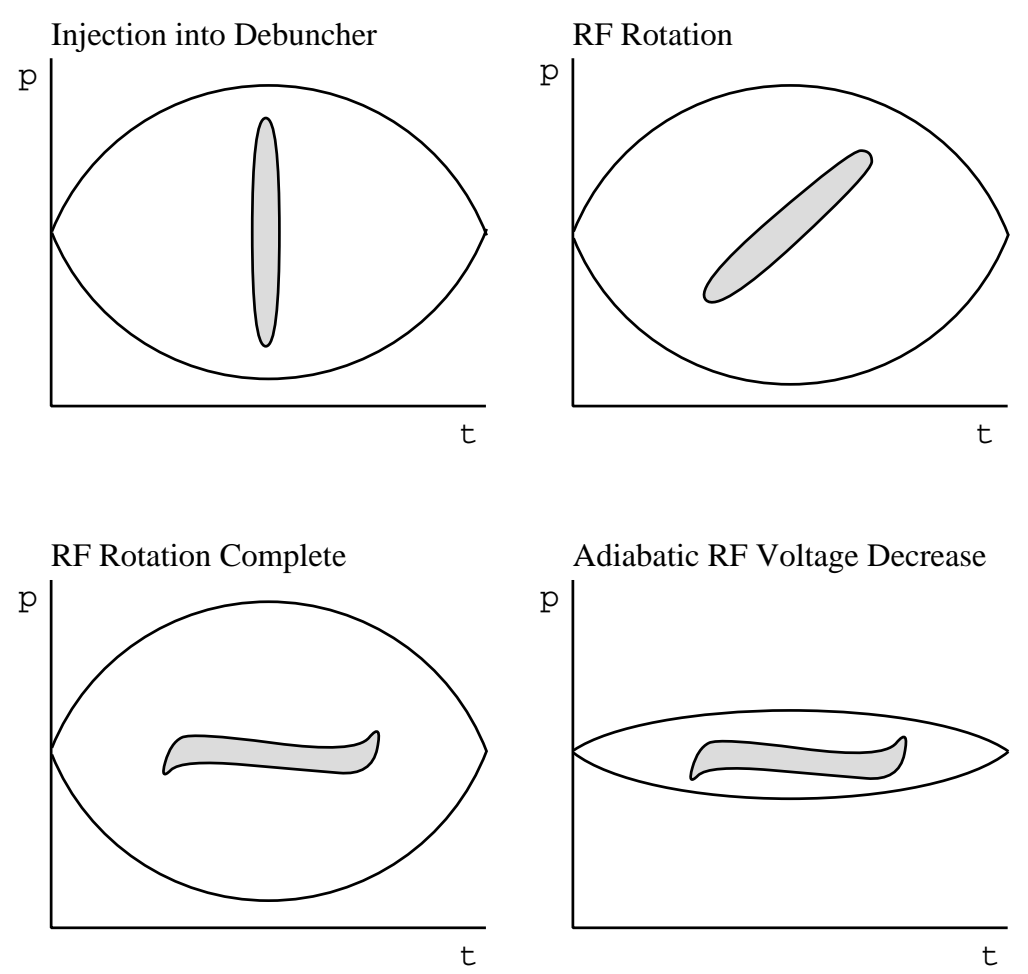

Figure 4.3: Schematic showing the RF rotation of a $\bar{p}$ bunch (shaded area) after injection into the Debuncher, and adiabatic matching of the RF bucket (outer curves), as described in the text, and in Ref. [28].

rotates in phase in such a way that longitudinal phase space $(\propto \Delta p \Delta t)$ is conserved. After $90^{\circ}$ of rotation in RF phase, (see Fig. 4.3) the RF voltage is adiabatically decreased, reducing $\Delta p / p$ for the bucket to the $\Delta p / p \sim 0.2 \%$ momentum spread of the rotated (and now debunched) beam. The process of matching the bunch to to the RF bucket in which it sits requires approximately $0.1 \mathrm{~ms}$, or about 53 orbits in the Debuncher. [28] Finally, before transfer to the Antiproton Accumulator, some transverse and longitudinal beam cooling is performed to reduce the momentum spread to $\Delta p / p \simeq 0.09 \%$. 


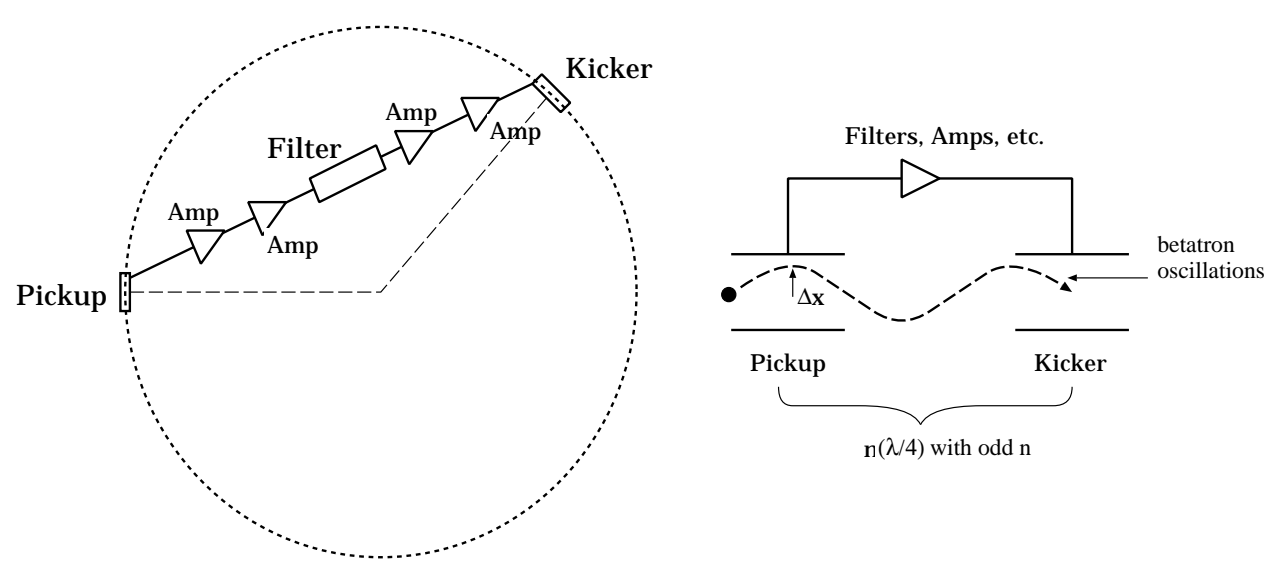

Figure 4.4: Schematic of a stochastic cooling system.

This cooling is performed by use of the stochastic cooling system, a system without which accumulation of intense $\bar{p}$ beams would be impossible. The concept underlying a stochastic cooling system is simple. (see Fig. 4.4) Both transverse and longitudinal cooling systems will be explained briefly here.

\subsubsection{Transverse Stochastic Cooling}

As a particular $\bar{p}$ passes one of several pickup electrodes positioned around the ring, its deviation $x$ from the central orbit is detected. A correction can then be applied by transmitting a signal to a kicker electrode, which is located an odd number of betatron oscillation quarter-wavelengths downstream from the pickup. The kick (which is timed so that it is delivered when the particle detected by the pickup arrives at the kicker) then causes the $\bar{p}$ to have a transverse position $x-g x$ at the pickup, the next time around, where $g$ is the "system gain". [29] For a single particle "beam", in principle, a single kick could be used to correct its orbit. 
However, since we are dealing with a beam of $\mathrm{N}$ antiprotons, each of which affects the motion of the others, the effect of each kick delivered is smaller. Further, the presence of the other particles means that the pickup detects the mean deviation of a portion of the beam, and delivers an appropriate kick to that portion of the beam. The effect of this, along with the fact that the system gain $g$ mentioned earlier cannot be exactly 1, is that cooling of the beam requires not one, but many, kicks. The cooling principle, though, is applicable for a beam of any size.

\subsubsection{Longitudinal Stochastic Cooling}

Transverse cooling, as described above, decreases the physical size of the beam by decreasing the amplitude of betatron oscillations - but this has only a marginal effect on the momentum distribution of the beam. For that purpose, longitudinal, or momentum, cooling must be done.

In momentum cooling, it is necessary to detect variations $\Delta p$ from the mean, or central, beam momentum $\langle p\rangle$. The mechanism for momentum cooling is similar in nature to that for transverse cooling. In this case, however, a band-pass or "notch" filter is used in the pickup-kicker network, so that particles nearest the central frequency are the least affected. That is, the presence of the filter allows a positive correction to the slightly low frequency particles, and a negative kick to the slightly high frequency ones, while leaving the particles near the central orbit frequency alone.

Transverse cooling in the Debuncher reduces the emittance of the $\bar{p}$ beam from $\sim$ $20 \pi$ to $\sim 7 \pi$ mm-mrad. Longitudinal cooling reduces $\Delta p / p$ from the $\sim 0.2 \%$ achived 
by the RF manipulations to $\sim 0.09 \%$. At this point, the $\bar{p}$ beam is transferred into the Accumulator Ring, and the Debuncher is ready to accept a new $\bar{p}$ batch.

\subsection{3 $\bar{p}$ Stacking in the Accumulator - Preparations for Res- onance Scanning}

The stacking of $\bar{p}$, i.e., building a large beam from many individual batches, requires a special application of longitudinal cooling. In the Antiproton Accumulator, there are two longitudinal cooling systems, which operate at slightly different frequencies (and therefore, momenta). These are known as the "stack-tail" and "core" cooling systems. $\bar{p}$ bunches are injected from the Debuncher ring with a momentum $p_{i n j}$, while the main part (or core) of the stack in the Accumulator orbits with a slightly lower momentum $p_{\text {core }}$. After a bunch is injected into the Accumulator, (every two seconds) the "stack-tail" pickups and kickers act to lower the momentum of the injected bunch, from $p_{i n j}$ to $p_{c o r e}$, bringing it into the slightly smaller core radius orbit of the stack. This must be completed within two seconds, before the next batch arrives. At the same time, the "core" cooling system reduces the momentum spread $\Delta p_{\text {core }}$ of the "core" of the stack. (See Fig. 4.5)

During each of these two second accumulation cycles, the $\bar{p}$ stack intensity increased by approximately $2 \times 10^{7} \bar{p}$. For the purposes of E835, an initial stack intensity of $50-80 \times 10^{10} \bar{p}$ was desired, so that on average, stacking required approximately $15-25$ hours. After stacking was completed, $\bar{p}$ production was halted, and some further longitudinal stochastic cooling was done, bringing the final $\Delta p / p$ down to approximately $0.02 \%$. At this point, control of the beam was handed over to the E835 experimenters. 

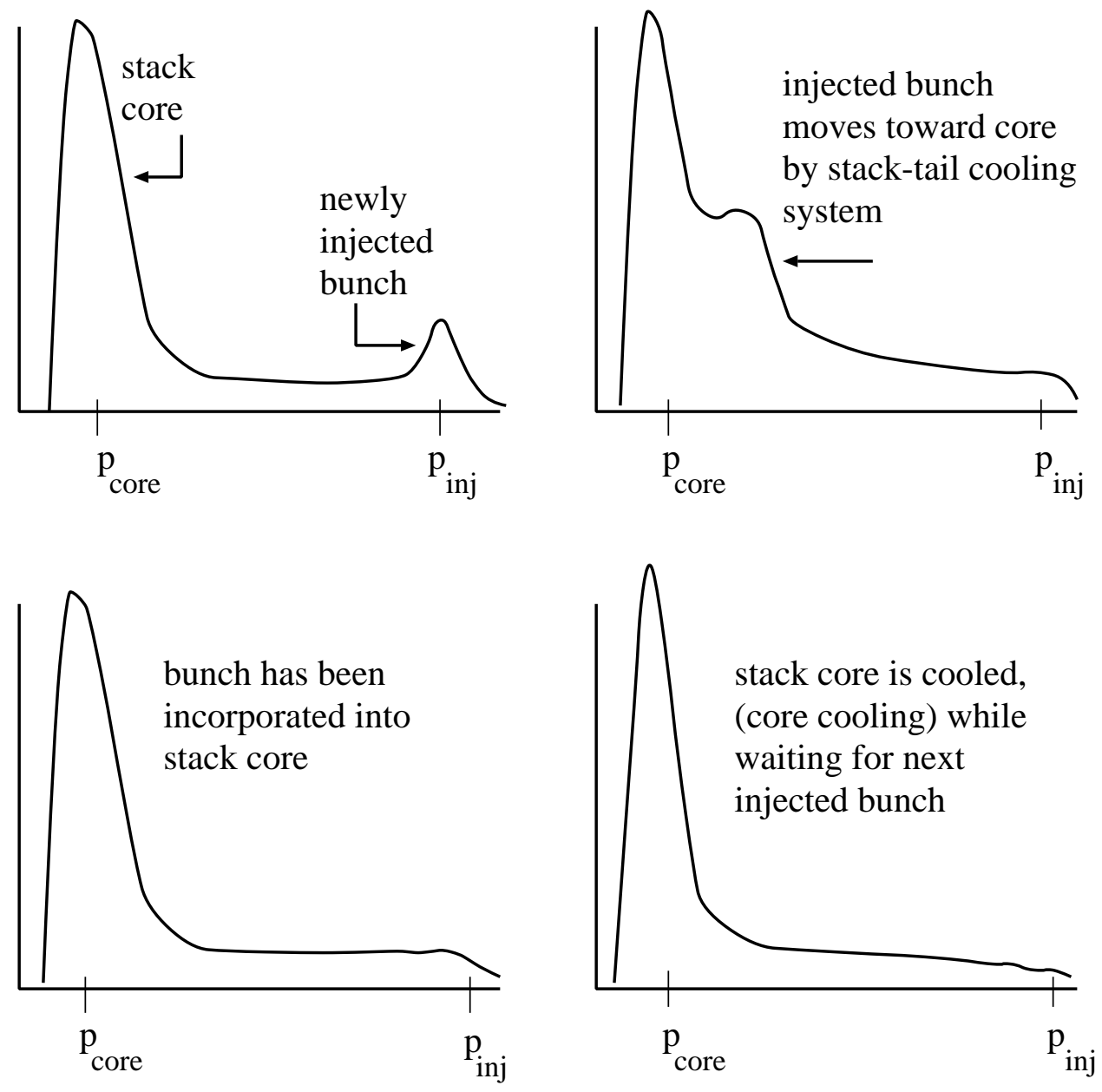

Figure 4.5: Schematic showing the cycle of $\bar{p}$ bunch injection and longitudinal cooling of the stack in the Accumulator. 
Table 4.1: Masses of charmonium states and the necessary $\bar{p}$ beam momenta. The mass given for $\eta_{c}^{\prime}$ corresponds to the center of the range searched in E835.

\begin{tabular}{|l|cc|}
\hline$c \bar{c}$ State & Mass $\left(\mathrm{GeV} / \mathrm{c}^{2}\right)$ & $p_{\text {beam }}(\mathrm{GeV} / \mathrm{c})$ \\
\hline$\eta_{c}$ & 2.9848 & 3.6919 \\
$J / \psi$ & 3.0969 & 4.0657 \\
$\chi_{0}$ & 3.4152 & 5.1931 \\
$\chi_{1}$ & 3.5105 & 5.5502 \\
${ }^{1} P_{1}$ & 3.5262 & 5.6099 \\
$\chi_{2}$ & 3.5562 & 5.7246 \\
$\eta_{c}^{\prime}$ & 3.6180 & 5.9639 \\
$\psi^{\prime}$ & 3.6861 & 6.2321 \\
\hline
\end{tabular}

In order to form charmonium states resonantly, it is necessary to decelerate the $\bar{p}$ beam from the stacking momentum of $\sim 8.9 \mathrm{GeV} / \mathrm{c}$ to an appropriate momentum for the state under study. (See Table 4.1) Deceleration of the beam is performed by a gradual ramping down of the voltage in the accumulator magnets. The minimum step size in the $\bar{p}$ beam momentum, $\sim 150 \mathrm{keV} / \mathrm{c}$, is determined by the least significant bit in the magnet power supplies. This corresponds to a minimum step size in $\sqrt{s}$ of $\sim 50 \mathrm{keV}$. For all but $J / \psi$, whose width is $\leq 100 \mathrm{keV}$, this step size is sufficiently small to perform a several-point scan across the resonance as depicted schematically in Figure 4.6.

A typical resonance scan was begun by decelerating the $\bar{p}$ beam so that the corresponding CM energy $\sqrt{s}=\sqrt{\left(2 m_{p}^{2}+2 E_{l a b} m_{p}\right)}$ was comfortably above the resonance mass plus a few half-widths. The gas jet target (see Section 4.3) is turned on, and the data was recorded for a certain time interval. This cycle was repeated by successively stepping the $\bar{p}$ beam energy down, so that the full excitation curve was scanned. The complete scan of a resonance typically required more than one 


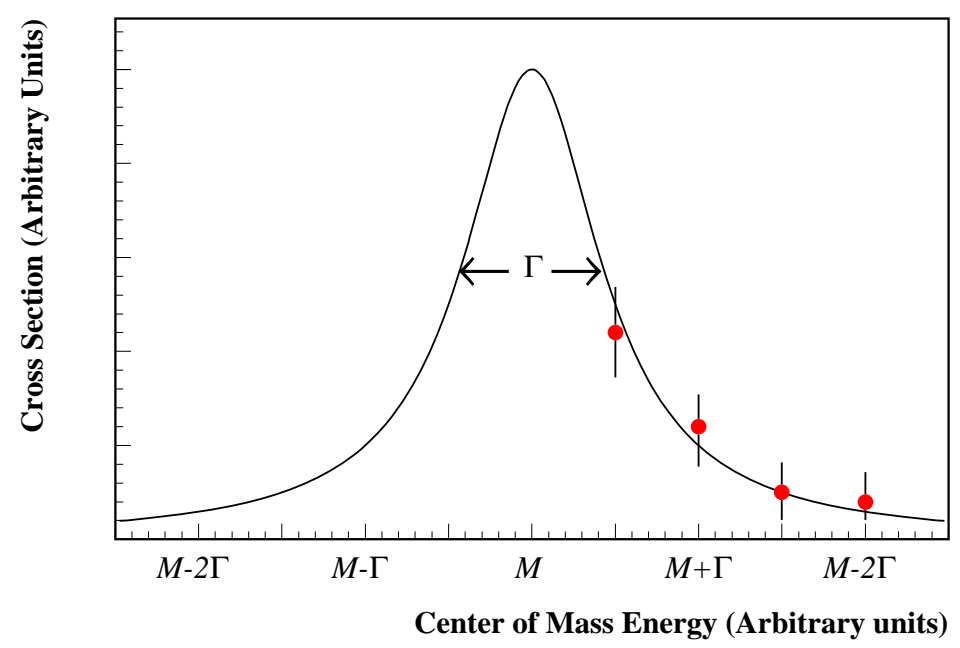

Figure 4.6: Hypothetical scan of a resonance, illustrating the procedure described in the text. Four data points have been taken in this "scan".

stack.

The resonance parameters of the charmonium state under investigation are then found by fitting the measured cross sections $\sigma$ (the number of events divided by the integrated luminosity $\int \mathcal{L}$, acceptance $\alpha$ and efficiency $\epsilon_{i}$ ) at each energy step to the convolution of a Breit-Wigner lineshape $\sigma_{B W}$ and the beam energy distribution $g\left(E-E_{0}\right)$, and the contribution from background processes $\sigma_{b k g}$ :

$$
\sigma(E)=\frac{N(E)}{\left[\int \mathcal{L}\right]_{i} \epsilon_{i} \alpha}=\left(\int_{0}^{\infty} g_{\text {beam }}\left(E-E_{0}\right) \sigma_{B W}(E) d E+\sigma_{b k g}\right) .
$$

The Breit-Wigner resonance parameters are determined from the best fit. 


\section{$4.2 \quad \bar{p}$ Beam Energy Measurement}

Since we form states of charmonium at resonance, and measure their widths and masses by scanning the beam energy across each resonance, precise measurements

of the $\bar{p}$ beam energy $(E)$ and the beam energy spread $\Gamma_{\text {beam }}(\mathrm{FWHM})$ are of critical importance, especially for states whose total widths are comparable to $\Gamma_{\text {beam }}$.

We determine the energy of the $\bar{p}$ beam by making measurements of its revolution frequency and of the orbit length $L$. The beam velocity $\beta$ in an orbit of length $L$ is determined by:

$$
\beta c=f L=f\left(L_{0}+\Delta L\right)
$$

where $f$ is the revolution frequency, $L_{0}$ is the reference orbit length and $\Delta L$ the deviation from $L_{0}$. The antiproton total energy is $m_{p} \gamma=m_{p} \sqrt{1 / 1-\beta^{2}}$, and the center of mass energy is

$$
\sqrt{s}=\left[2 m_{p}^{2}\left(1+\sqrt{\frac{1}{1-\beta^{2}}}\right)\right]^{1 / 2} .
$$

\section{Measuring Orbital Frequency: Schottky Noise}

The frequency $f$ is determined by measuring the Schottky noise spectrum of the beam current. Schottky noise can be defined qualitatively as the electromagnetic noise generated by microscopic (i.e. individual particle) fluctuations in a current of charged particles. From the spectral power density of the Schottky noise we can derive a frequency spectrum for the $\bar{p}$ beam, and thus the beam energy distribution.

Consider a single charged particle in a circular orbit, as depicted in Fig. 4.7. The "current" corresponding to this particle (of charge q), measured by an elec- 


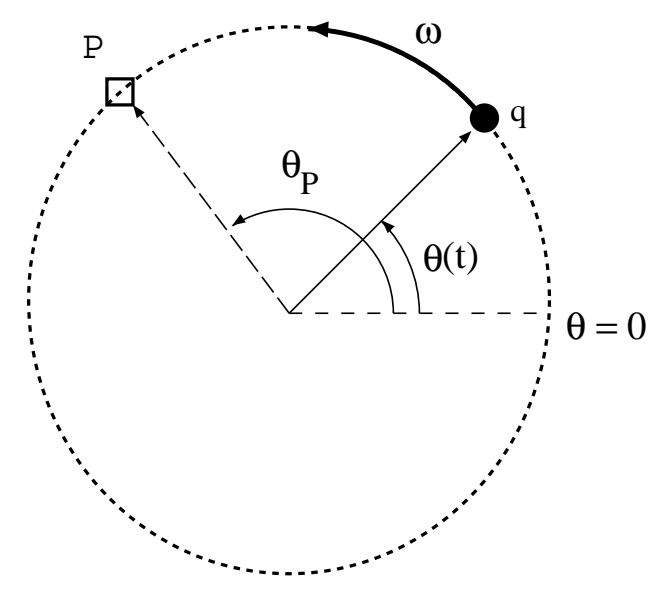

Figure 4.7: Schematic of a single particle carrying charge $q$, travelling in a circular orbit with angular frequency $\omega$, at position $\theta(t)$. At point $P$, (angle $\left.=\theta_{P}\right)$ there is an electromagnetic pickup circuit.

tromagnetic pickup $\mathrm{P}$ at angle $\theta_{P}$ (the phase angle in the orbit), may be written as

$$
I_{P}(t)=q \sum_{m=-\infty}^{+\infty} \delta\left[\theta(t)-\theta_{P}-2 \pi m\right] .
$$

The current $I_{P}(t)$ is the sum of an infinite number of delta functions corresponding to the times at which the particle passes the pickup at $\theta_{P}$.

We can express the delta functions as functions of time, and take the Fourier transform to examine the frequency domain. $\theta(t)$ is simply equal to $\omega t$, where we have defined $\theta=0$ as the position of the particle at time $t=0$. Similarly, $\theta_{P}$ is equal to $\omega$ times the time $t_{P}$ which it takes the particle to reach $\theta_{P}$.

Thus we can rewrite Eq. 4.4 as

$$
I_{P}(t)=q \sum_{m=-\infty}^{+\infty} \delta\left[t-t_{P}-m T\right] .
$$

If we take the Fourier transform of the above equation, we can derive the current in 
the frequency domain (with $\omega_{0}=2 \pi / T$ ):

$$
\begin{aligned}
I_{P}(\omega) & =\int_{-\infty}^{+\infty} I_{P}(t) e^{-i \omega t} d t \\
& =\frac{q \omega_{0}}{2 \pi} e^{-i \omega t_{p}} \sum_{m=-\infty}^{+\infty} \delta\left(\omega-m \omega_{0}\right) .
\end{aligned}
$$

If we extend our consideration to a beam of $\mathrm{N}$ particles, we find that the single particle delta functions are smeared into "Schottky bands" of frequencies $\Omega=m\left(\omega_{0} \pm \Delta \omega\right)$, (or, equivalently, $\left.m\left(f_{0} \pm \Delta f\right)\right)$ due to deviations in individual particle frequencies. In practice, we may observe Schottky band of the $m$ th harmonic, and by measuring the power density spectrum, determine both $f_{0}$ and $\Delta f$. From $f_{0}$, we can calculate the central CM energy for our interactions, and from $\Delta f$ the distribution of $\mathrm{CM}$ energies.

The Schottky spectrum in the Antiproton Accumulator is measured by a coaxial quarter-wavelength electromagnetic pickup which has a resonant frequency of 79.323 MHz. Depending on the beam energy, the pickup measures the 127th, 128th or 129th harmonic of the central orbit frequency. This spectrum is acquired from the pickups by a spectrum analyzer, and written to disk every 3 minutes.

A typical 3-minute Schottky spectrum is shown in Fig. 4.8. The mean frequency of the spectrum is determined to better than 1 part in $10^{7}$ - the spread $\Gamma(f)$ is taken to be the FWHM spread of the spectrum. These quantities are related to the mean energy $E$ and energy spread $\Gamma(E)$ of the $\bar{p}$ beam by equations 4.2 and 4.3 . 


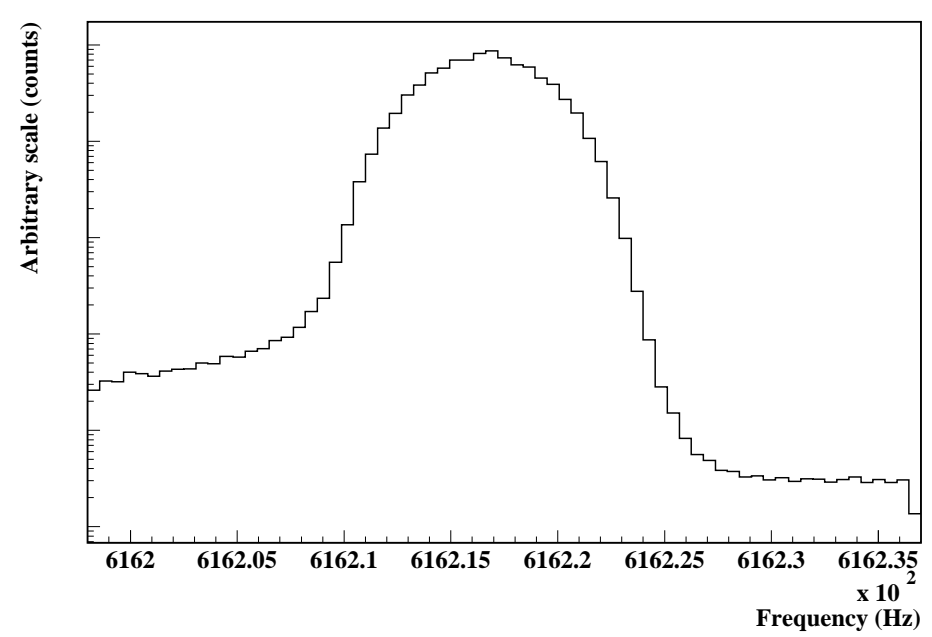

Figure 4.8: Semilog plot of the Schottky frequency distribution from a run at $\sqrt{s}=$ $3.101 \mathrm{GeV}$.

\section{Measuring the Orbit Length: $\psi^{\prime}$ Mass}

The orbit length $L$ can be determined in principle by physical survey, but not sufficiently accurately for our needs. However, there is an alternative. Since the $\psi^{\prime}$ mass has been measured by the resonance depolarization method at Novosibirsk to be $M\left(\psi^{\prime}\right)=3686 \pm 0.1 \mathrm{MeV},[30]$ a scan of the $\psi^{\prime}$ resonance may be used to determine the length of the orbit very precisely.

By scanning the $\psi^{\prime}$ resonance, we are able to determine the frequency $f_{0}$ which corresponds to the peak of the $\psi^{\prime}$ cross section. The antiproton momentum which corresponds to $\sqrt{s}=3.686 \mathrm{GeV}$ is $p_{\text {beam }}=6.2317 \mathrm{GeV}$. This corresponds to $\beta_{0}=$ 
0.98885. We can thus determine the orbit length:

$$
L_{0}=\frac{\beta_{0} c}{f_{0}} .
$$

The error $\delta L_{0}$ in the measured orbit length $L_{0}$ may be expressed in terms of the error $\delta M_{\psi^{\prime}}$ in the mass of the reference state, $\psi^{\prime}$.

$$
\delta L_{0}=\frac{c}{f_{0}} \delta \beta_{0}-\frac{\beta_{0} c}{f_{0}^{2}} \delta f_{0} .
$$

As mentioned earlier, $\delta f_{0} \approx 10^{-7}$, and therefore the second term can be safely neglected. Further,

$$
s_{0}=M_{\psi^{\prime}}^{2}=m_{p}^{2}+m_{\bar{p}}^{2}+2 m_{p} E_{\bar{p}}=2 m_{p}^{2}\left(1+\gamma_{0}\right) .
$$

Differentiating the above, we obtain

$$
2 M_{\psi^{\prime}} \delta M_{\psi^{\prime}}=2 m_{p}^{2} \beta_{0} \gamma_{0}^{3} \delta \beta_{0}
$$

or, from Eq. 4.9

$$
\delta L_{0}=\frac{c}{f_{0}} \frac{M_{\psi^{\prime}} \delta M_{\psi^{\prime}}}{m_{p}^{2} \beta_{0} \gamma_{0}^{3}}, \quad \text { or } \quad \frac{\delta L_{0}}{L_{0}}=\frac{M_{\psi^{\prime}} \delta M_{\psi^{\prime}}}{m_{p}^{2} \beta_{0}^{2} \gamma_{0}^{3}} .
$$

In E835 the reference orbit length was found by this method to be $L_{0}=474.0497 \mathrm{~m}$, with $\delta L_{0}= \pm 0.7 \mathrm{~mm}$.

\section{Energy Measurements}

During E835 operation, the $\bar{p}$ beam was rarely found to be exactly on the nominal reference orbit used for calibration. However, deviations from the reference orbit can be measured by a system of 48 Beam Position Monitors (BPMs), which measure 
horizontal displacements $\Delta x_{i}$ of the beam. The errors in measuring the $48 \Delta x_{i}$ translate to approximately an error in the overall length $\Delta L= \pm 1 \mathrm{~mm}$. Thus the overall error in determining the orbit length $L$ is $\sqrt{\delta L_{0}^{2}+\Delta L^{2}}=1.2 \mathrm{~mm}$.

As a result of this error in the orbit length $L$, the uncertainty in the energy in the center of mass $E_{c m}$ is given simply by Eq. 4.12 as:

$$
\partial E_{c m}=\frac{\partial L}{L} \frac{\gamma^{3} \beta^{2} m_{p}^{2}}{E_{c m}}=\left(2.532 \times 10^{-6}\right) \times\left(\frac{\left(E_{c m}^{2}-4 m_{p}^{2}\right)\left(E_{c m}^{2}-2 m_{p}^{2}\right)}{8 m_{p}^{4}}\right) \times E_{c m} .
$$

For example, the error in measuring the resonance mass of $2980.00 \mathrm{MeV}$ due to the above sources of error would be only $\delta M \approx 0.046 \mathrm{MeV}$.

\subsection{Hydrogen Gas Jet Target}

In E835 we form charmonium states via the annihilations of $p$ and $\bar{p}$ which occur at the intersection of the $\bar{p}$ beam in the Accumulator with a gas jet target of molecular hydrogen.

Typical gas targets require the passage of the incident beam through Mylar windows of a containment vessel, and are fine for extracted beam experiments. However, for internal gas targets in storage ring environments such as the Antiproton Accumulator, such a target is impractical. The loss of energy incurred in the repeated passage through even the thinnest Mylar windows would cause significant beam losses, and broadening of the beam energy distribution. Thus in a storage ring, an unconfined gas target, with high capacity vacuum pumping to minimize extraneous beam-gas interactions away from the interaction region, is required. In E760 and 
E835, we have utilized a hydrogen gas jet, which intersects the $\bar{p}$ beam at a right angle.

In our predecessor experiment, E760, the gas jet apparatus had only minimal capability for controlling the jet density, and the jet was kept at an approximately constant areal density of $\sim 5 \times 10^{13}$ atoms $/ \mathrm{cm}^{2}$. Typically, the $\bar{p}$ stack began with $\sim 40 \times 10^{10} \bar{p}$ circulating with an orbital frequency of $0.62 \mathrm{MHz}$, so that the initial instantaneous luminosity was approximately $5 \times 10^{13} \times 0.62 \times 10^{6} \times 40 \times 10^{10}=12 \times$ $10^{30} \mathrm{~cm}^{-2} \mathrm{sec}^{-1}$. As the antiproton beam was depleted by interactions, or by being scattered out of the accumulator acceptance, the luminosity steadily decreased.

In order to maximize the integrated luminosity for a given amount of $\bar{p}$ beam used, the gas jet target for E835 was modified to keep instantaneous luminosity nearly constant by automated adjustment of gas jet pressure and temperature. This is advantageous not only from a beam-usage standpoint, but it also minimizes rate-dependent corrections to detector efficiencies by keeping the interaction rate approximately constant.

The E760 gas jet was redesigned such that its density could be varied from about $1 \times 10^{13}$ to $3.2 \times 10^{14}$ atoms $/ \mathrm{cm}^{2}$. For beam currents of 10 to about 80 $\times 10^{10} \bar{p}$, this range of densities made it possible to maintain a constant luminosity of $\sim 2 \times 10^{31} \mathrm{~cm}^{-2} \mathrm{~s}^{-1}$. The new gas jet target, as modified for E835, is described in detail in Ref. [31]. A schematic of the modified E835 jet target is presented in Fig. 4.9.

The $\mathrm{H}_{2}$ gas is injected through a thin nozzle $(\sim 37 \mu \mathrm{m}$ at its narrowest point) at temperatures from $\sim 20-100 \mathrm{~K}$ and pressures from $\sim 20-100$ p.s.i. The jet 


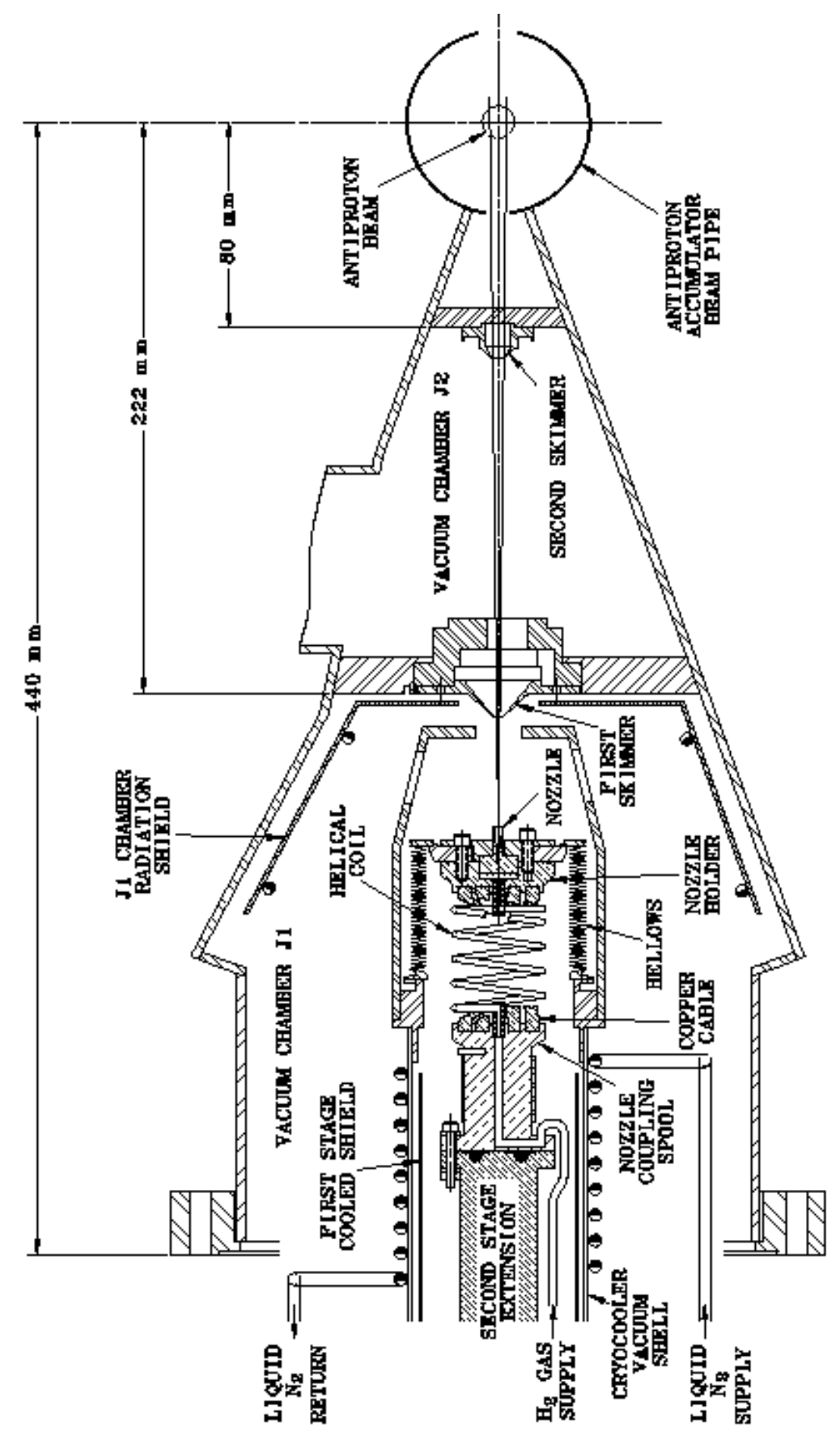

Figure 4.9: Schematic illustration of the E835 Gas Jet target. 


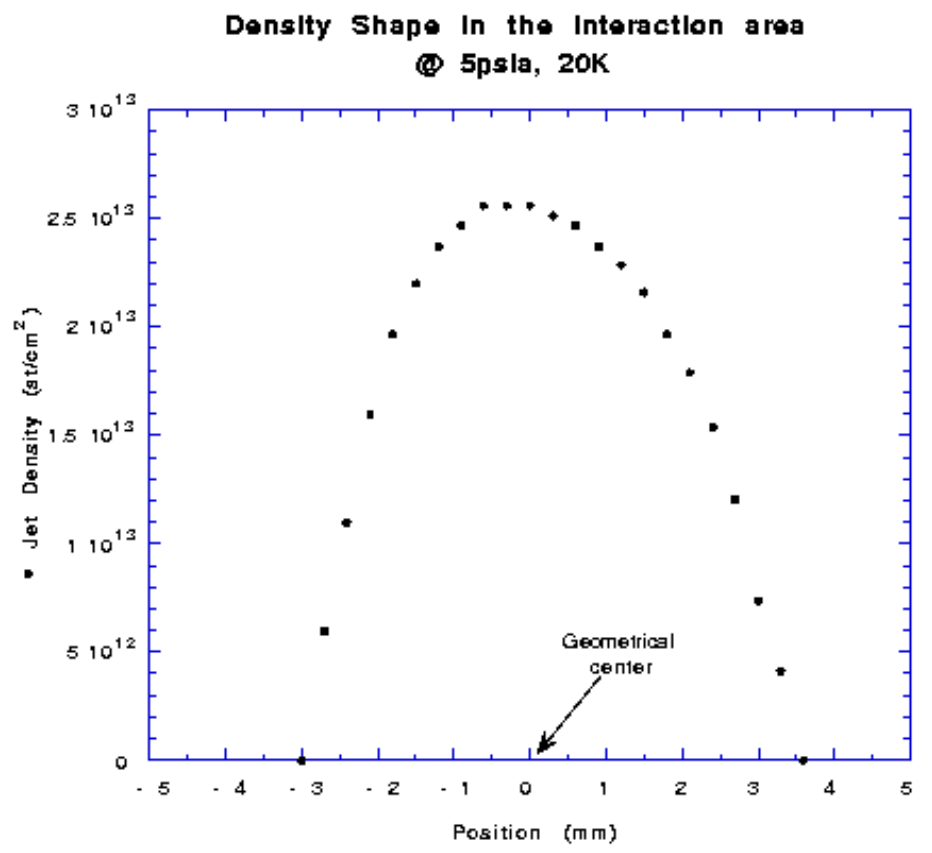

Figure 4.10: Measured gas jet density distribution (in the longitudinal direction, $z$, in the interaction region. The observed asymmetry in the distribution is not understood. [32]

of $\mathrm{H}_{2}$ travels at supersonic speeds through the convergent-divergent nozzle, forming a supersaturated jet of molecular clusters, each cluster containing up to $10^{7}-10^{8}$ $\mathrm{H}_{2}$ molecules. A series of collimators reduces the transverse size of the gas jet to approximately $6.5 \mathrm{~mm}$. Fig. 4.10 shows the measured gas jet density distribution in the $z$ (or beam) direction. 


\subsection{The E835 Detector}

The E835 detector is a non-magnetic spectrometer, azimuthally symmetric about the beam axis. It has a large acceptance $(\sim 50-60 \%$ for two-body final states at center of mass energies accessible to this experiment) and is specifically optimized for the identification of electromagnetic final states such as $e^{+} e^{-}, e^{+} e^{-} \gamma$ and $\gamma \gamma$.

The central region of the detector is composed of three major parts: an inner set of detectors used for charged particle tracking and triggering, a segmented threshold Čerenkov detector for the identification of electrons, and a large 1280-element lead glass calorimeter (CCAL). In addition, luminosity is measured by a set of solid state detectors placed at an angle of approximately $90^{\circ}$ with respect to the beam direction. In the forward direction, two different calorimeters (FCAL I and II) were used at different times during the running of E835. The first was a set of 144 lead-scintillator sandwich counters which was used in E760, while the second consisted of a new set of 144 lead glass counters. Neither FCAL was used in the analysis presented in this dissertation, and they will not be described here in detail. A schematic of the E835 detector system layout is presented in Fig. 4.11.

\subsubsection{Inner detectors}

There are 7 inner detectors in the E835 detector system. A vertical slice through these detectors perpendicular to the beam axis is shown in Fig. 4.12. From the innermost to the outermost, these are: a scintillator hodoscope $H 1$, a straw tube drift chamber $\mathrm{SC} 1$, a second scintillator hodoscope $\mathrm{H} 2^{\prime}$, a silicon pad detector SI, 


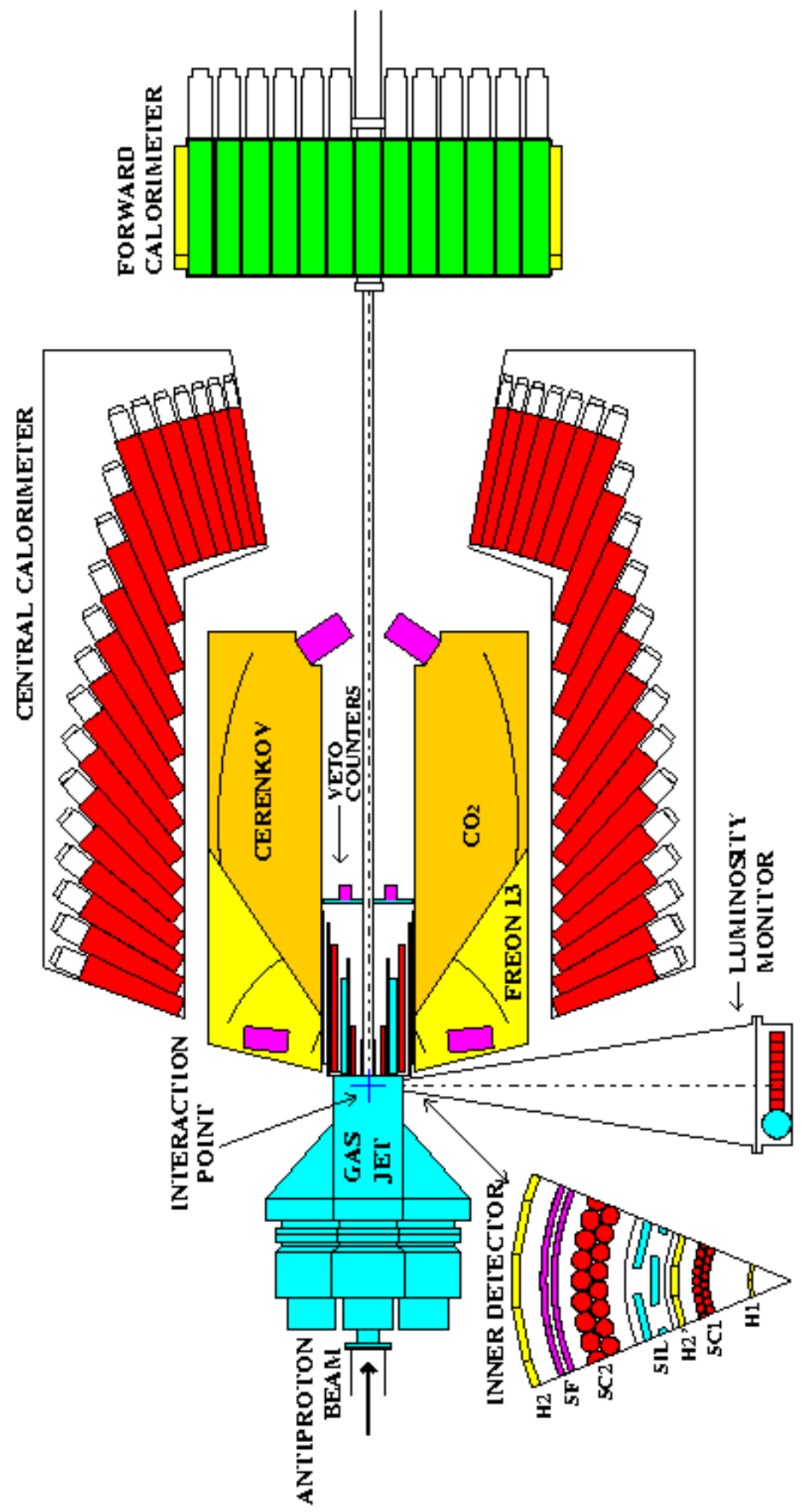

Figure 4.11: Schematic of the E835 detector system 


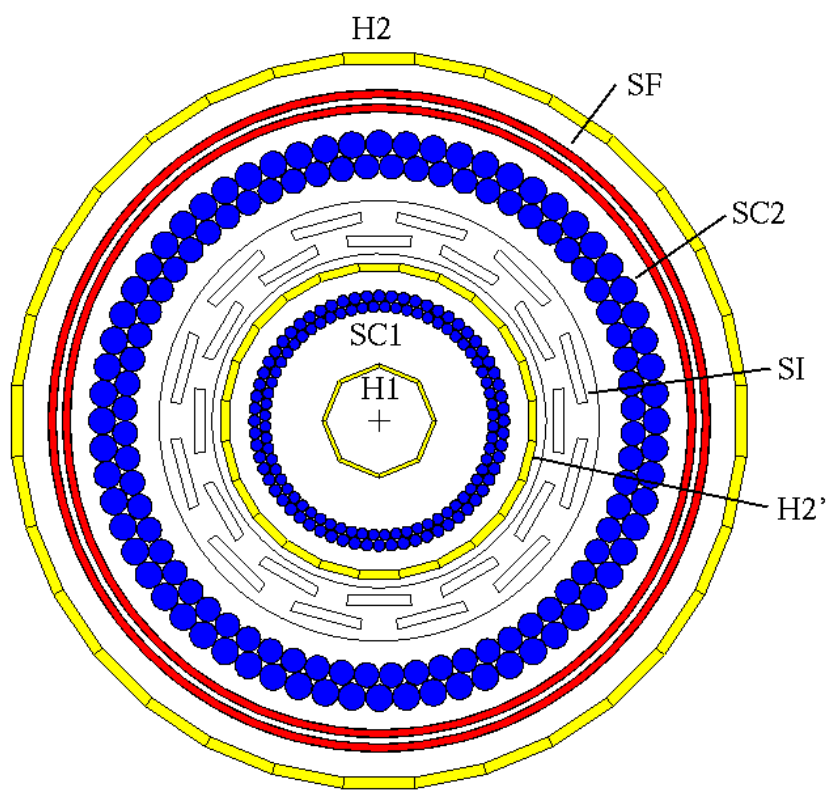

Figure 4.12: Schematic of the inner detectors, from the "beam's eye" perspective. From inside to outside, they are $H 1, \mathrm{SC} 1, \mathrm{H} 2{ }^{\prime}, \mathrm{SI}, \mathrm{SC} 2, \mathrm{SF}$ and $\mathrm{H} 2$, as designated in the text.

a second set of straws SC2, 2 layers of scintillating fibers SF, and finally a third scintillator hodoscope $H 2$. At the downstream end of this cylindrical array of inner detectors is a forward hodoscope FCH. Each of these subsystems is briefly described below.

\section{Scintillator Hodoscopes}

Each of the three scintillator hodoscopes, $H 1, H 2$ and $\mathrm{H}^{\prime}$, is composed of a number of rectangular Bicron 408 scintillators symmetrically arranged about the beam axis. Their primary function is to detect charged particles and produce fast trigger signals; secondarily they are used to reject events with unwanted charged tracks. 
In the following discussion, pulse heights are presented in terms of MIP units, i.e. they have been normalized in order to account for the difference in photoelectrons per minimum ionizing particle (MIP) among the different counters. The average number of photoelectrons per MIP is 50-75 for $H 1$, and 20-30 for both $H 2$ and $H 2^{\prime}$.

$H 1$ consists of eight $2 \mathrm{~mm}$ thick scintillator paddles covering the full azimuth and the polar angle $\theta$ from $9^{\circ}$ to $65^{\circ}$. The center of each paddle lies at a radius of $2.2 \mathrm{~cm}$ from the center of the beam pipe. Each paddle is individually light-tight and coupled to its own light guide and Phillips XP2982 PMT. The H1 signals are read out by both ADCs and TDCs, and discriminated signals are used in the first level charged trigger.

$H 2$ consists of thirty-two $4 \mathrm{~mm}$ thick scintillator paddles covering the full azimuth and, in $\theta$ from $15^{\circ}$ to $65^{\circ}$. The center of each $H 2$ element lies at a radius of $16.5 \mathrm{~cm}$ from the center of the beam pipe, and is coupled to light guides and Phillips XP2982 PMTs in the same manner as is $H 1$.

In E760 $H 1$ and $H 2$ were used in the charged trigger. They were oriented, however, in such a way that every fourth crack in $H 2$ was aligned in $\phi$ with a crack in H1. This resulted in about a $20 \%$ inefficiency for triggering in the immediate vicinity of the $H 1$ cracks. [33] It was decided, therefore, to add for E835 a third scintillator hodoscope with a different segmentation to avoid this problem. This detector, called $\mathrm{H}_{2}{ }^{\prime}$, consists of twenty-four $4 \mathrm{~mm}$ thick paddles which are positioned at a radius of $6.8 \mathrm{~cm}$, covering (like $H 1$ ) from $9^{\circ}$ to $65^{\circ}$ in $\theta$. It uses Hammamatsu R1398 PMTs, but in other respects it is identical to both $H 1$ and $H 2$.

The pulse height spectra from $H 1, H 2^{\prime}$ and $H 2$ are shown in Fig. 4.13. In 

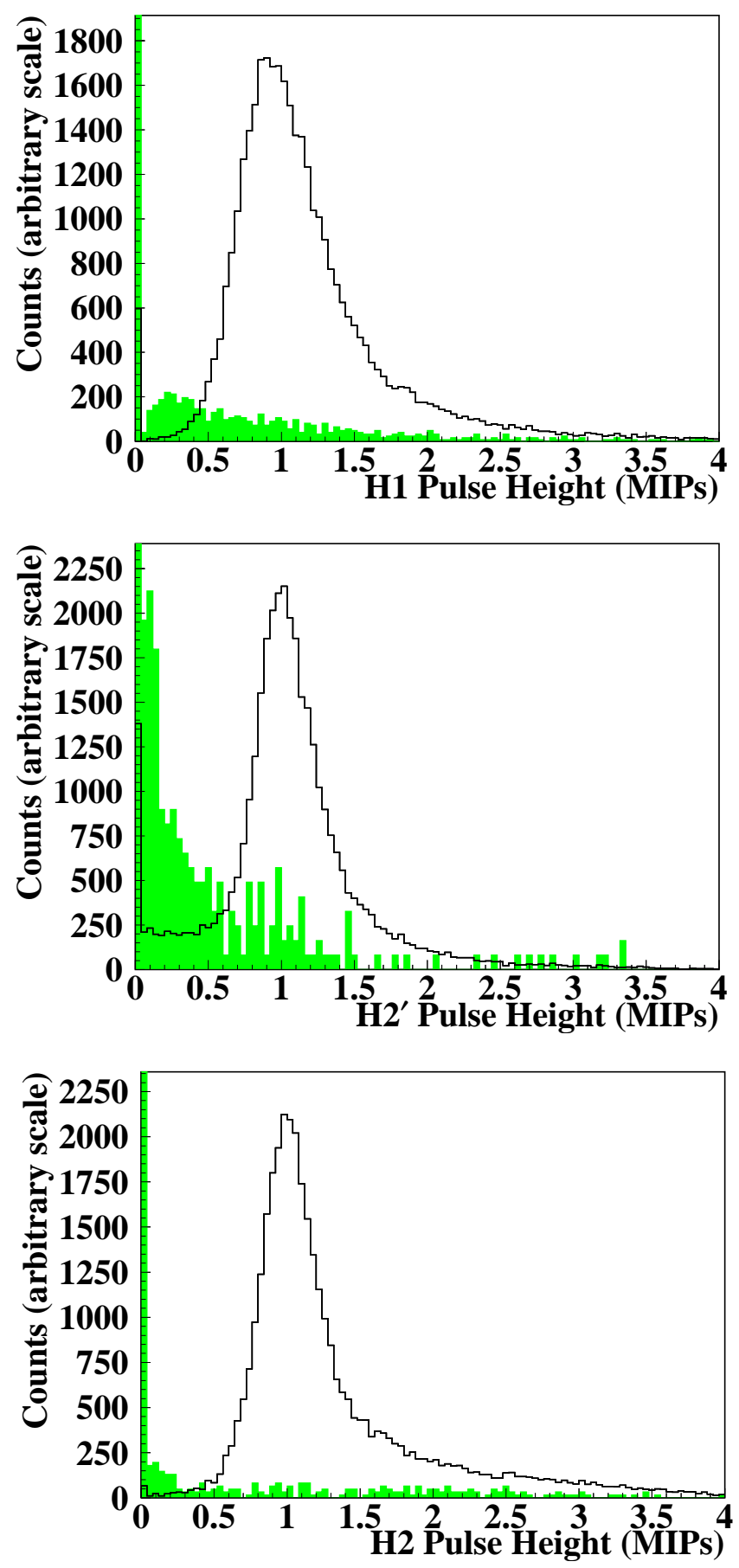

Figure 4.13: Pulse height spectra from (top) $H 1$, (middle) $H 2^{\prime}$ and (bottom) $H 2$ for electrons (unshaded histograms) and photons (shaded histograms). 
each plot the unshaded histogram shows the pulse height spectrum due to electrons, from kinematically-selected $J / \psi \rightarrow e^{+} e^{-}$events. The shaded histogram in each plot shows the pulse height spectrum of hodsocope paddles through which photons from $\pi^{0} \pi^{0} \rightarrow(\gamma \gamma)(\gamma \gamma)$ events pass (according to the angles $\phi$ for the photons, determined by the CCAL). The pulse height was plotted for an event only if the signal in the corresponding Čerenkov cell was negligible. Therefore, the shaded histograms truly show the noise level in each hodoscope, and not pulses due to $e^{ \pm}$or $e^{+} e^{-}$conversion pairs.

The Forward Charged Hodoscope (FCH) is a set of eight flat scintillator paddles which cover $\theta$ from approximately 2 to $10^{\circ}$. The purpose of the $\mathrm{FCH}$ is to veto events which have charged particles in the forward direction. The FCH may also be used to determine whether energy deposits observed in the forward calorimeter are due to charged particles or to photons.

The FCH paddles are each placed perpendicular to the beam pipe, at the end of the cylindrical arrangement of $H 1, H 2^{\prime}$ and $H 2$ hodoscopes. Each paddle covers approximately $50^{\circ}$ in azimuth, so that there is $\sim 5^{\circ}$ of overlap between adjacent paddles. For the triggers in which we expect to observe the $\gamma \gamma$ final state, the FCH is used in veto mode.

\section{Scintillating Fibers}

The scintillating fiber tracker is a detector intended primarily for high resolution measurement of the polar angle $\theta$ of charged particles. The 860 individual fibers (740 $\mu \mathrm{m}$ diameter Kuraray SCSF-3HF-1500) in the tracker are arranged in two 
barrel-like layers at radii of 14.40 and $15.06 \mathrm{~cm}$. Each fiber is laid as a circular loop on a cylindrical acrylic frame, with one end of the loop thermally spliced to a $4 \mathrm{~m}$ long clear fiber which carries the scintillation light to arrays of Visible Light Photon Counters (VLPCs). Each VLPC array consists of 8 VLPC pixels. 16 arrays are housed in a cassette, and 12 of these cassettes (total capacity $=8 \times 16 \times 12=1536$ pixels) were installed in a $6.5 \mathrm{~K}$ liquid-helium-cooled cryostat.

VLPCs, rather than PMTs, were chosen for their very high quantum efficiency, which is necessary to compensate for the low light output of the very thin scintillating fibers. An average number of $\sim 14$ photoelectrons per MIP was measured for each fiber. The signals (amplified by QPA02 cards) from the VLPC arrays were read out by custom-built NIM modules. Analog outputs from the NIM modules were sent to 11-bit LeCroy-LR4400 FERAs. More complete descriptions of this detector and its performance can be found in Refs [34]. The scintillating fiber tracker was not used in analysis of the data presented in this dissertation.

\section{Straw Tubes}

Two straw tube chambers, described in Ref. [35], lie at radii of 5.4 and $12.0 \mathrm{~cm}$. Each chamber consists of two cylindrical layers of straw tubes, each of which lie parallel to the beam axis. Their purpose is to provide both $\theta$ and $\phi$ measurements for charged particles. The azimuthal angle $\phi$ is determined by measuring drift time within the tubes, while $z$, the distance along the straw tube, is determined by charge division. Information from the straw tubes was not used in the analysis presented in this dissertation. 


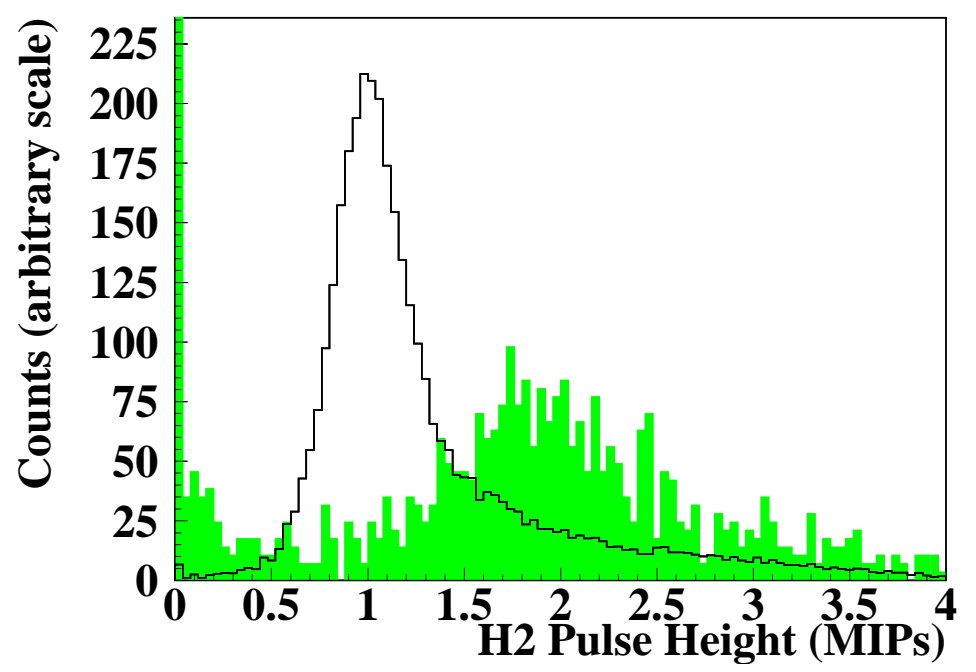

Figure 4.14: Pulse height spectrum for the hodoscope H2. The shaded histogram shows events in which there is no signal in the corresponding elements of hodscopes $H 1$ or $H 2^{\prime}$. The events centered around 2 MIPs show evidence of gamma conversions in the Silicon Barrel. For comparison, the unshaded histogram is the arbitrarily normalized $H 2$ spectrum for single electrons from $J / \psi \rightarrow e^{+} e^{-}$.

\section{Silicon Barrel}

A cylindrically symmetric array of 4608 silicon pads, [36] arranged on twenty-four printed boards at a mean radius of $9 \mathrm{~cm}$, was installed in E835. This detector failed to work properly, and therefore constituted $3.5 \%$ of a radiation length, on average, of dead material. The adverse effect of the silicon barrel was to provide undesirable conversions. If we examine the $H 2$ pulse height spectrum for CCAL clusters with no signal in $H 1$ or $H 2^{\prime}$, we clearly see a 2-MIP peak in the distribution. (See Fig. 4.14) We interpret these 2-MIP signals as evidence for photons which have converted in the silicon barrel. A further discussion of conversions, and their possible detrimental 
effect on $\gamma$ detection, is presented in Sec. 6.2.2.

\subsection{2 Čerenkov Detector}

The threshold Čerenkov detector [37] was built to identify electrons. It consists of eight wedge-shaped sections, which are aligned azimuthally with the eight paddles of $H 1$. Each Čerenkov section is divided into a backward cell $\left(\sim 34^{\circ}\right.$ to $\left.\sim 65^{\circ}\right)$ containing Freon-13 at atmospheric pressure $(n=1.000720)$, and a forward cell (from $\sim 15^{\circ}$ to $\left.\sim 38^{\circ}\right)$ with one atmosphere of $\mathrm{CO}_{2}(n=1.000410)$. (See Fig. 4.15)

The threshold energy for production of Čerenkov light by a $\pi^{ \pm}$in a medium with index of refraction $n$ is given by Since

$$
E_{t h r}\left(\pi^{ \pm}\right)=m_{\pi} \sqrt{\frac{n^{2}}{n^{2}-1}}
$$

Therefore, the pion energy threshold is $3.7 \mathrm{GeV}$ in the backward (Freon-13) cells, and $4.9 \mathrm{GeV}$ in the forward $\left(\mathrm{CO}_{2}\right)$ cells. The maximum pion energy expected in the $\bar{p} p \rightarrow \pi^{+} \pi^{-}$at the largest mass charmonium resonance investigated in $\mathrm{E} 835$, i.e., $\psi^{\prime}(3686)$ is $2.67 \mathrm{GeV}$ in the backward cells $\left(\right.$ at $\theta=38^{\circ}$ ) and $4.68 \mathrm{GeV}$ in the forward cells $\left(\right.$ at $\theta=15^{\circ}$ ). Thus only electrons are expected to produce Čerenkov light in either the forward or backward cells.

The Čerenkov light produced in the each forward cell is focused on to the PMT by a single ellipsoidal mirror, while in the backward cells light is focused onto the PMT by means of spherical and flat mirrors. The photoelectron yield in the forward cells is $\sim 14$ to 16 per incident electron, and in the backward cells is $\sim 7$ to 11 . The pulse height for the Čerenkov counter is shown in Fig. 4.16. It is expressed 

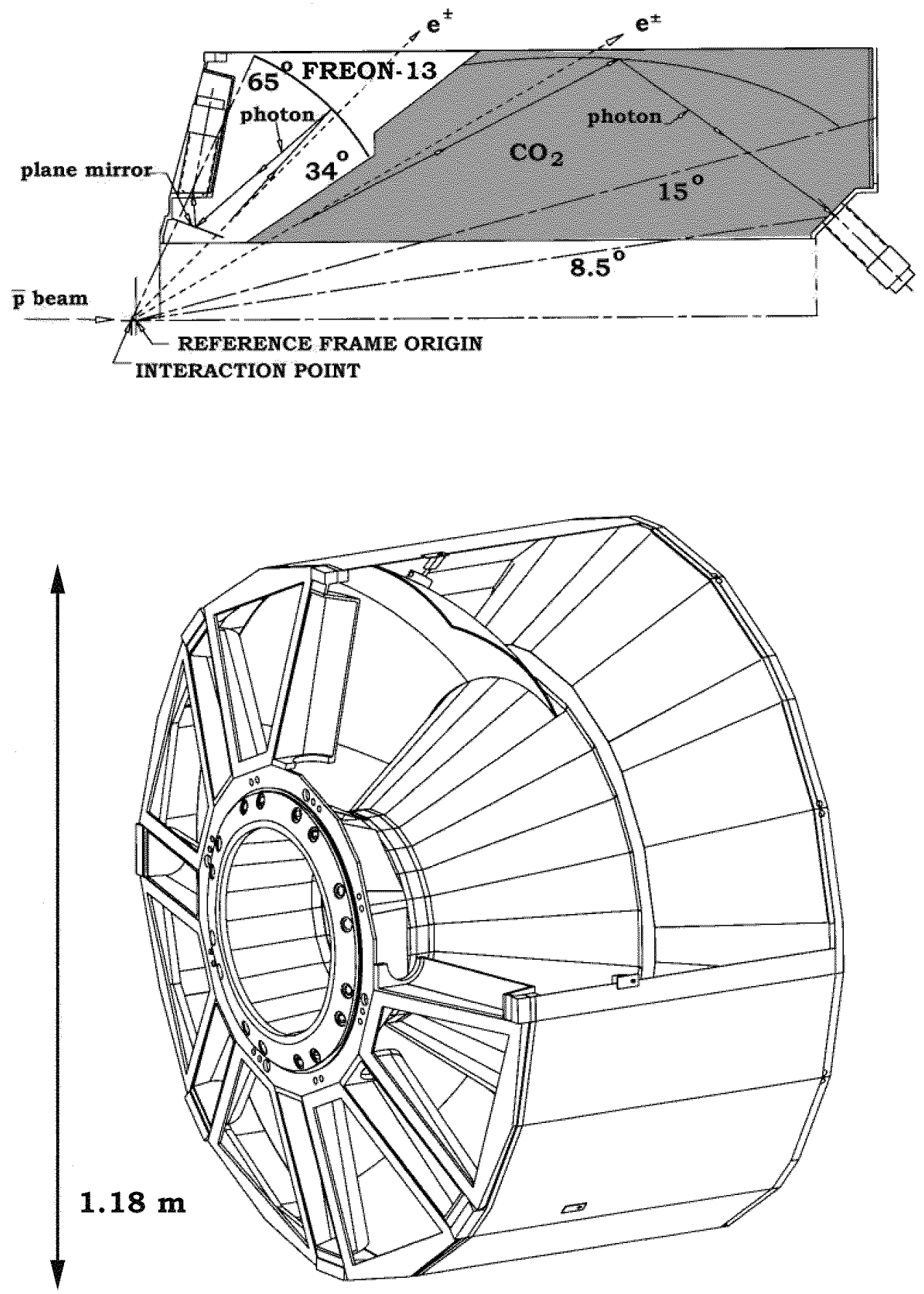

Figure 4.15: Schematics of the Cerenkov detector, (top) showing one octant and its mirrors and angles, and (bottom) an isometric view of the eight backward-angle cells. 


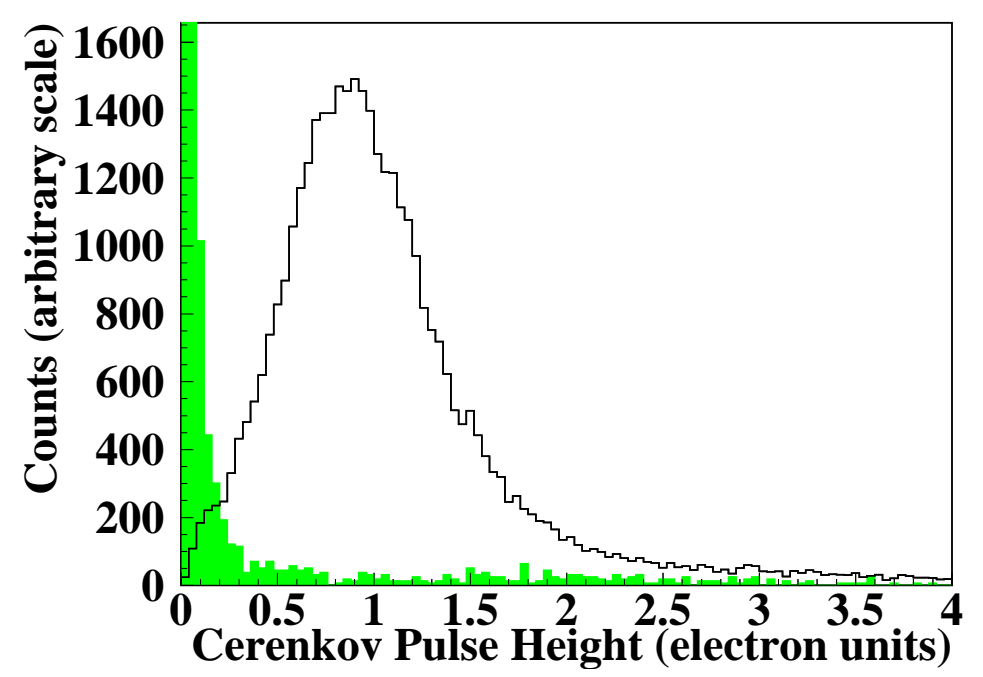

Figure 4.16: Normalized signal from the Čerenkov counter to electrons (unshaded histogram) and photons (shaded histogram). The pulse height is expressed in units in which a value of $\sim 1$ is expected for an electron.

in 'electron' units, in which a pulse height of 1 is expected for an electron. The unshaded histogram shows the pulse height in the Cerenkov counter due to single electron tracks (taken from a sample of $J / \psi \rightarrow e^{+} e^{-}$events selected kinematically). The shaded histogram in Fig. 4.16 shows the pulse height in the Čerenkov cells for photons from $\pi^{0} \pi^{0} \rightarrow(\gamma \gamma)(\gamma \gamma)$ events. In order to illustrate the level of electronic and other noise in the Čerenkov detector, we show only signals from photons which have not converted (as determined by the pulse height in the corresponding $\mathrm{H} 2$ paddle).

The septum dividing forward and backward cells at $38^{\circ}$ in the E760 Cerenkov was replaced in E835 with a split septum with part at $34^{\circ}$ and part at $38^{\circ}$. This replacement was made in order to ensure that both $e^{-}$and $e^{+}$from a $J / \psi \rightarrow e^{+} e^{-}$ 


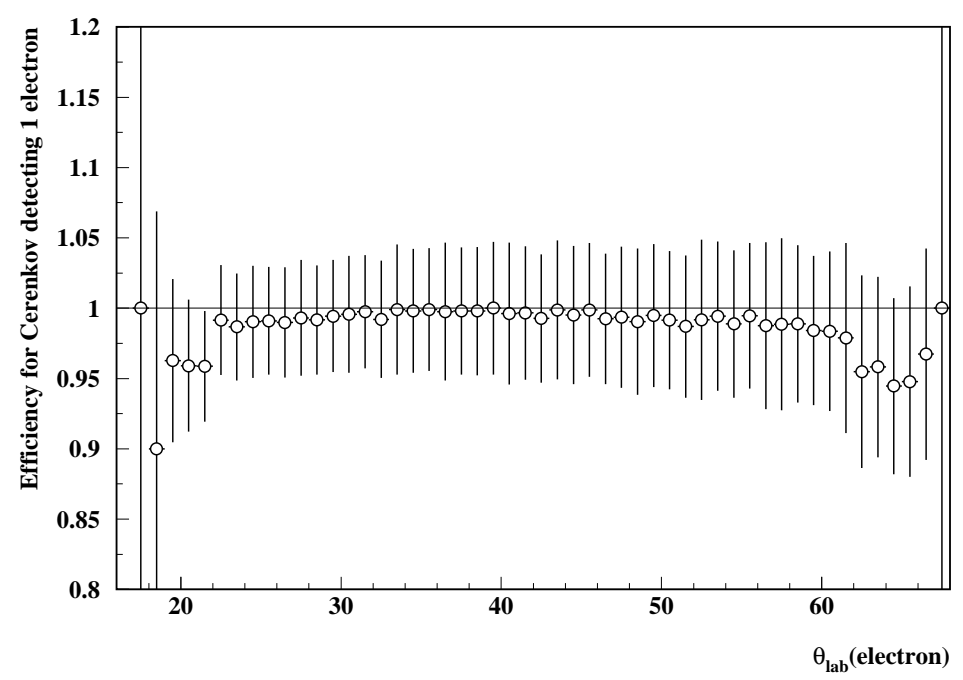

Figure 4.17: Efficiency for Čerenkov detection of at least 1 electron from $J / \psi \rightarrow e^{+} e^{-}$ events as a function of $\theta_{l a b}\left(e^{-}\right)$.

or $\psi^{\prime} \rightarrow e^{+} e^{-}$would never be simultaneously incident upon the septi. In E760 this problem gave rise to $\mathrm{a} \sim 30 \%$ inefficiency for detection of one electron at angles $\theta_{\text {lab }} \approx 38^{\circ}$, and $\sim 80 \%$ inefficiency for detection of both at $\approx 38^{\circ}$. [38] The design of the new split septum has corrected this inefficiency. (See Fig. 4.17) The single electron efficiency over the central part $\left(\sim 22^{\circ}\right.$ to $\left.60^{\circ}\right)$ of the polar angle subtended by the Čerenkov is $(98.1 \pm 0.5) \%$. [39]

\subsubsection{Central Calorimeter}

The Central Calorimeter (CCAL) is the workhorse of the E835 detector system. It is an azimuthally symmetric array of 1280 lead glass (Schott F2 type, $L_{R}=3.13 \mathrm{~cm}$ ) Čerenkov counters arranged in 20 "rings" (each ring has a common central polar 
angle $\theta$ ) and 64 "wedges" (each wedge has a common central azimuthal angle $\phi$ ). Complete coverage is achieved in $\phi$, while the $\theta$ range covered is $10.5^{\circ}$ to $70^{\circ}$. Each of the blocks in a wedge points directly back to the interaction point, and range in length from $38 \mathrm{~cm}\left(12 L_{R}\right)$ in ring 1 (largest $\left.\theta\right)$ to $50 \mathrm{~cm}\left(16 L_{R}\right)$ for rings 8 through 20 (smallest $\theta$ ). These lengths provided substantial energy containment (90-95\% of total).

Schematics of the CCAL showing its division into rings and wedges are presented in Figs. 4.18 and 4.19. Table 4.2 lists the angles $\theta$ corresponding to each ring. Each wedge subtends an angle $\Delta \phi=360^{\circ} / 64=5.625^{\circ}$. The granularity of the calorimeter segmentation was chosen so that photons from symmetric $\pi^{0}$ decays at the highest possible $\pi^{0}$ energy foreseen could be resolved as two separate photons. The resolution of such photon pairs will be discussed in detail in Sec. 6.1.

The 64 CCAL wedges are constructed as individual units: the 20 blocks which make up a wedge are contained in a thin stainless steel box. (See Fig. 4.20) The sides of each wedge box are $0.735 \mathrm{~mm}$ thick (so that wedges are separated by $2 \times 0.735=$ $1.470 \mathrm{~mm}$ of inactive material), and each block within the wedge is separated by a $0.245 \mathrm{~mm}$ thick stainless steel partition. While the existence of the inert interstitial material complicates reconstruction of energies and positions, it is necessary from a structural standpoint.

Both electrons and photons generate electromagnetic showers in lead glass. The shower composition is the same: electron-positron pairs created by pair production interactions, and photons from bremsstrahlung, and Čerenkov radiation. Ultimately, 


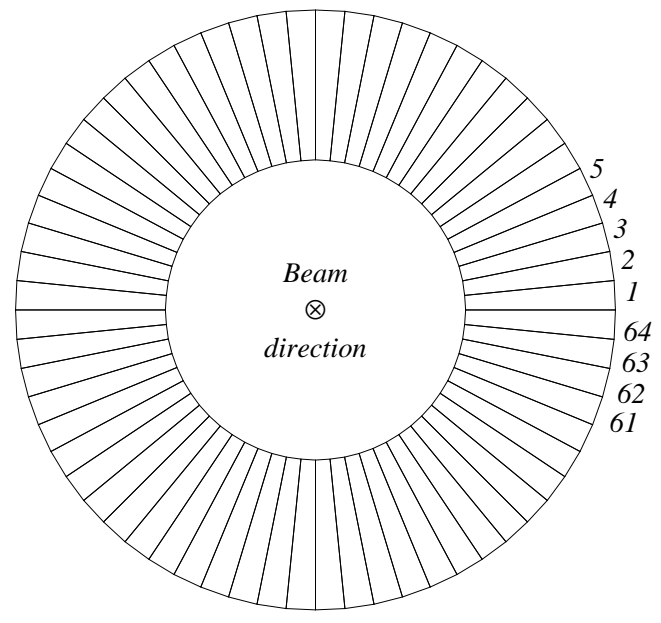

Figure 4.18: Schematic of the CCAL, azimuthal view.
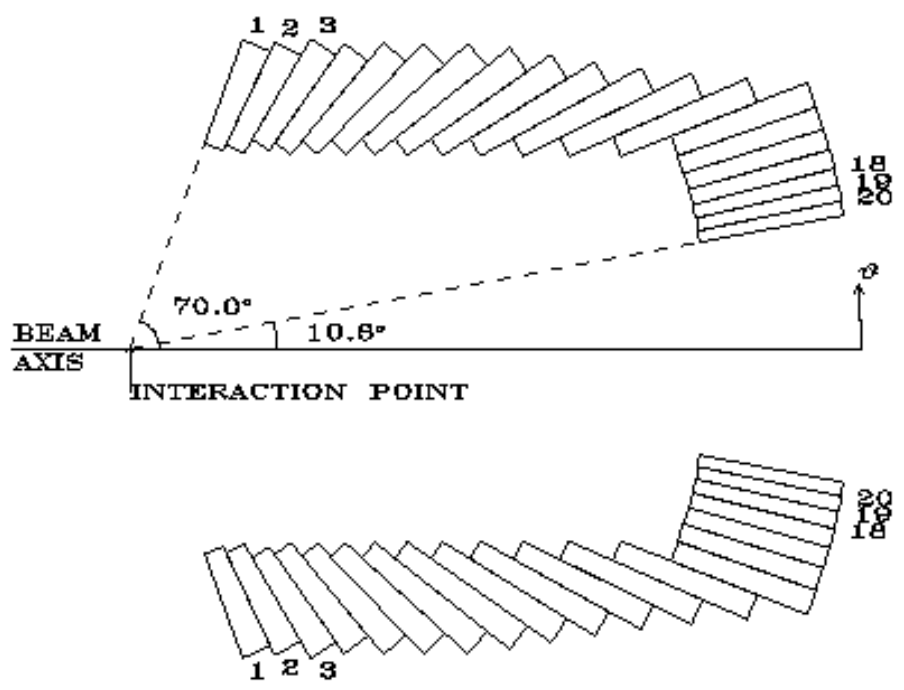

Figure 4.19: Schematic of the CCAL, lateral view. Two wedges are shown. 
Table 4.2: Characteristics of the 20 CCAL rings.

\begin{tabular}{l|clll}
\hline $\begin{array}{l}\text { Ring } \\
\text { Number }\end{array}$ & $\begin{array}{c}\text { Distance } \\
(\mathrm{mm})\end{array}$ & $\begin{array}{c}\text { Block Length } \\
(\mathrm{mm})\end{array}$ & $\begin{array}{l}\theta_{\max } \\
\text { degrees }\end{array}$ & $\begin{array}{l}\theta_{\min } \\
\text { degrees }\end{array}$ \\
\hline 1 & 724.35 & 378.01 & 70.00 & 64.77 \\
2 & 758.67 & 386.49 & 64.77 & 59.74 \\
3 & 800.67 & 398.78 & 59.74 & 54.94 \\
4 & 850.79 & 415.02 & 54.94 & 50.39 \\
5 & 909.64 & 435.44 & 50.39 & 46.10 \\
6 & 977.86 & 460.26 & 46.10 & 42.10 \\
7 & 1056.17 & 489.79 & 42.10 & 38.37 \\
8 & 1145.42 & 500.00 & 38.37 & 34.92 \\
9 & 1246.56 & 500.00 & 34.92 & 31.74 \\
10 & 1360.65 & 500.00 & 31.74 & 28.81 \\
11 & 1488.90 & 500.00 & 28.81 & 26.13 \\
12 & 1632.64 & 500.00 & 26.13 & 23.68 \\
13 & 1793.39 & 500.00 & 23.68 & 21.45 \\
14 & 1972.82 & 500.00 & 21.45 & 19.42 \\
15 & 1972.87 & 500.00 & 19.42 & 17.57 \\
16 & 1972.92 & 500.00 & 17.57 & 15.89 \\
17 & 1972.96 & 500.00 & 15.89 & 14.37 \\
18 & 1972.99 & 500.00 & 14.37 & 12.99 \\
19 & 1973.01 & 500.00 & 12.99 & 11.74 \\
20 & 1973.04 & 500.00 & 11.74 & 10.61 \\
\hline
\end{tabular}

photons from the shower are collected at the back of each block by optically coupled Hammamatsu PMTs.

The lead glass blocks in various CCAL rings are of different sizes. Several different PMT types are therefore used, in order to provide coverage of the maximum area of the back of each block by the PMT (and thus capture the maximum amount of Čerenkov light). The blocks in rings 1 through 14 have 3" PMTs (R3036-02) attached; those in rings 15 and 16 have 2.5" PMTs (R3345-02); 17 and 18, 2" PMTs 


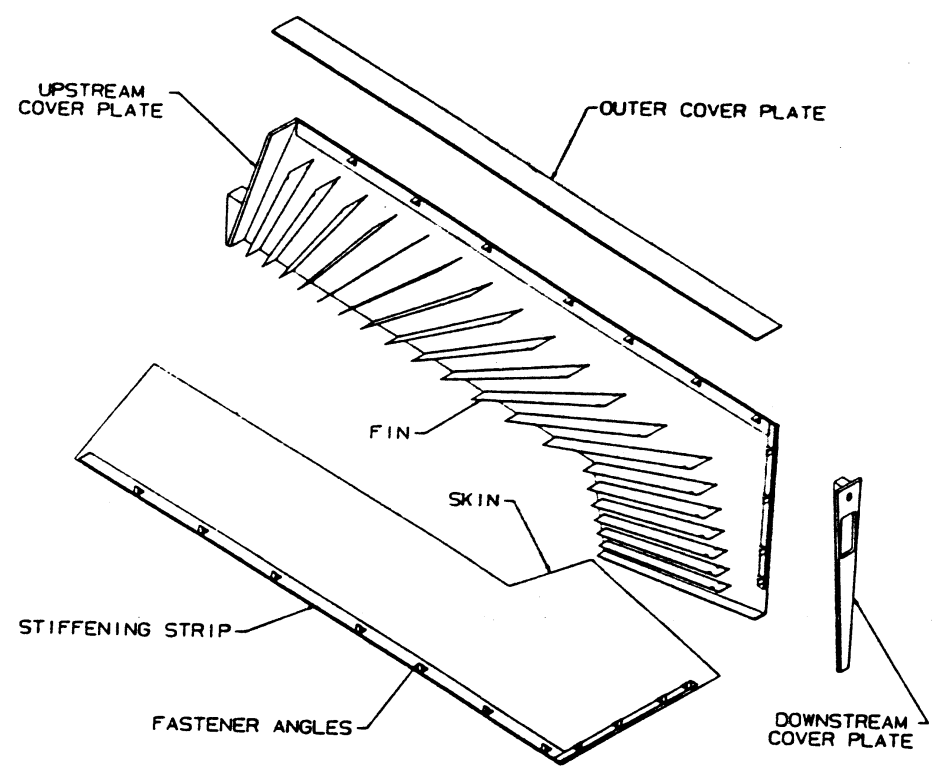

Figure 4.20: Schematic showing the internal construction of a CCAL wedge. The "fins", which separate blocks within a wedge, are .254 mm steel, while the "skins", are $.735 \mathrm{~mm}$ thick - so that blocks in a ring are separated by $2 \times .735=1.47 \mathrm{~mm}$.

(R2154-04); 19 and 20, 1.5" PMTs (R580-13). A clear fiber optic cable is also attached to the back of each block in order to distribute light to each block from a laser monitoring system. The RG-174 signal cable from each PMT is passed to the back of the wedge box, where there is a block connector to which an external cable harness (also RG-174) is attached.

\section{CCAL Signal Shaping}

With the increased instantaneous luminosity in E835 compared to E760, event rates in all detectors were expected to increase by up to a factor five over typical rates from E760. These event rates could not be handled by the CCAL readout, if the 
150 ns width of the FERA gates used in E760 was left unchanged.

It was decided that in E835 the CCAL signals should be shaped in a way such that we can use a FERA gate which is as short as possible (ultimately we decided upon $100 \mathrm{~ns}$ gates) in order to minimize the occurrence of multiple events within a single gate. This was done by means of the Splitter-Shaper-Discriminator Circuit (SSD), or simply, the shaper, whose schematic is presented in Figure 4.21. An example of an input and output pulse from the shaper circuit is shown in Fig. 4.22. More details regarding the shaper circuit, and its testing and performance, are given in Appendix A.

\section{Timing of CCAL Signals}

In E760 it was not possible or practical to instrument the CCAL with 1280 channels of TDC's so that timing information would be directly available for all CCAL signals. In E835, we were able to instrument the entire CCAL with TDC's. After the signal has been shaped by the SSD circuit, a small part of the signal is split off for the discriminator, whose output is sent to the TDC, while the rest is sent to the FERA. The discriminator had an individually adjustable threshold level, which was typically set to $6 \mathrm{mV}$, which corresponds to approximately $12 \mathrm{MeV}$ deposited in the CCAL block.

\section{CCAL Laser Monitor}

In order to monitor the status of individual CCAL blocks for sudden large gain shifts, a system using a Xenon flashlamp and fiber-optic coupling to each block was 

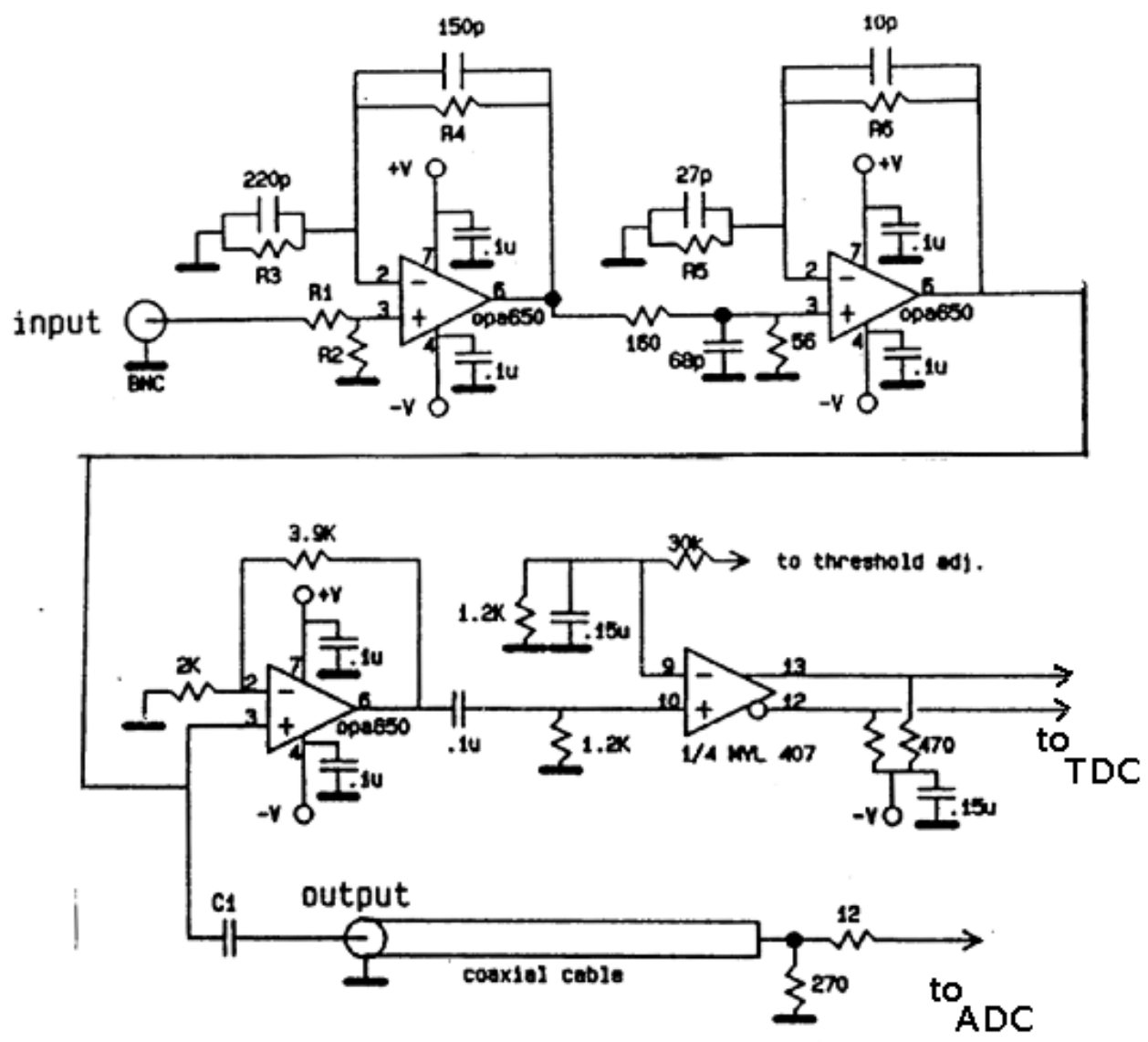

\begin{tabular}{|ccccccccl}
\hline Tube diameter & R1 & R2 & R3 & R4 & R5 & R6 & C1 \\
(3") & 1 & 24 & 27 & $1.2 \mathrm{~K}$ & $1.0 \mathrm{~K}$ & $1.3 \mathrm{~K}$ & $1.3 \mathrm{~K}$ & $22 \mathrm{nF}$ \\
(2") & 2 & 30 & 20 & $1.2 \mathrm{~K}$ & 680 & 820 & $1.5 \mathrm{~K}$ & $22 \mathrm{nF}$ \\
$\left(1.5^{\prime \prime}\right)$ & 3 & 30 & 18 & 910 & 680 & 680 & $1.5 \mathrm{~K}$ & $18 \mathrm{nF}$
\end{tabular}

Figure 4.21: Schematic diagram of an SSD channel for the shaping of the input signal from a CCAL block. 


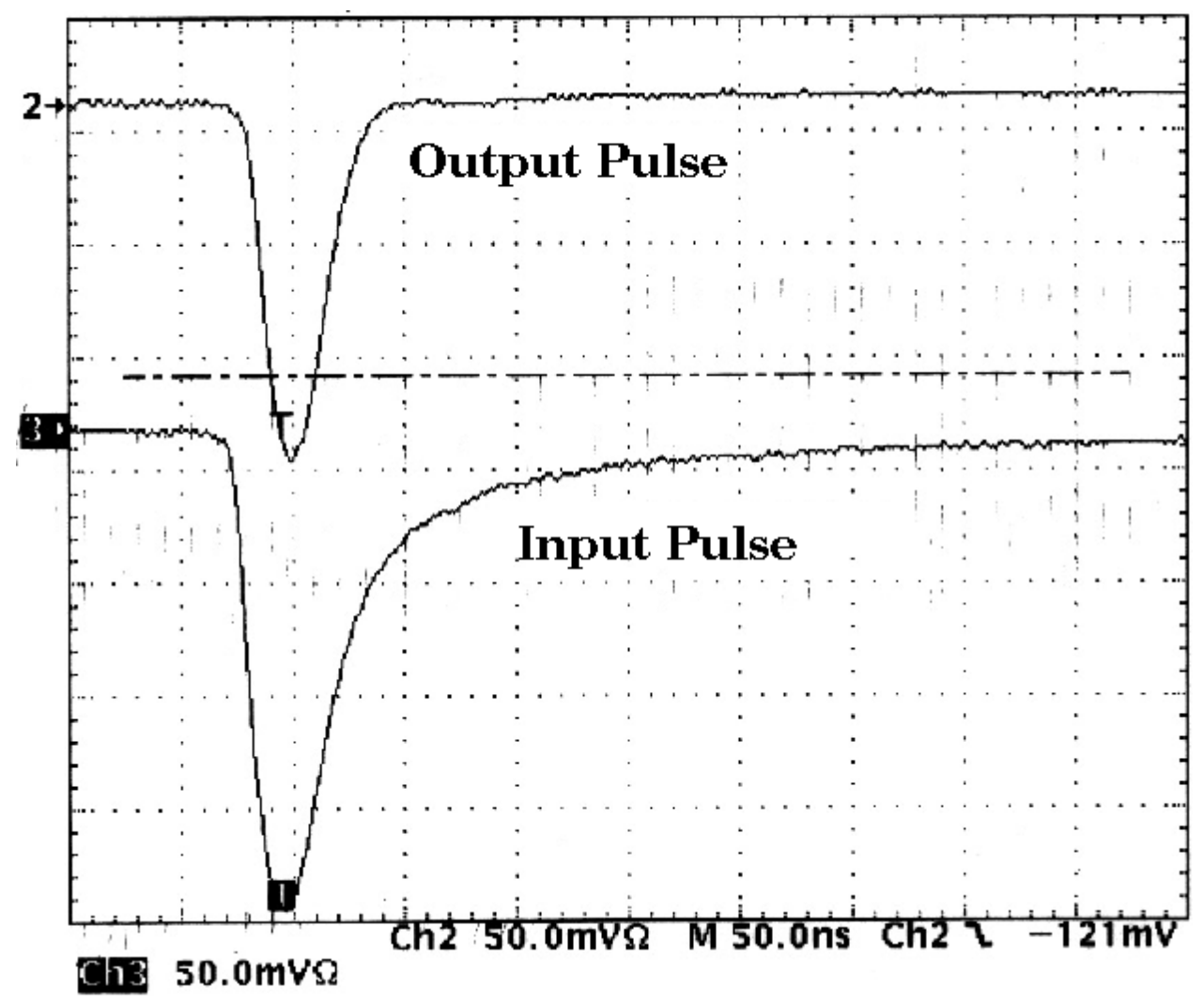

Figure 4.22: CCAL PMT pulses at the input and output of the signal shaper circuit. 
implemented in E760. [40] In E835, the flashlamp was replaced with a laser and scintillator system, which is illustrated in Fig. 4.23. A Laser Science, Inc. model \#VSL-337ND Nitrogen laser was contained in a light-tight enclosure with several separate chambers. (See Fig. 4.23) The UV light (peak wavelength of $337 \mathrm{~nm}$ ) from the laser was incident upon a piece of scintillator which produced visible light peaked in the blue part of the spectrum. In the chamber along with the scintillator were a pair of photodiodes which monitored the visible light output from the laser, for comparison to the response of the CCAL blocks.

The chamber also enclosed one end of a rectangular block of clear Lucite (the "mixing bar", an obvious misnomer) to which 64 fiber optic cables were glued. Blue light incident upon the mixing bar was transmitted through to the other end, where the fiber optic cables were attached. Approximately equal amounts of this light are transmitted via the fiber optic cables to each of the 64 CCAL wedges. Each of these 'external' fiber optic cables was connected at the back of the wedge enclosure to a short 'internal' fiber. Inside each wedge enclosure, the internal fiber was coupled to a smaller mixing bar which was used to divide the light transported to that particular wedge further into 20 parts, each of which is carried via a fiber optic cable to one of the 20 blocks in the wedge.

The laser system was preferred to the E760 flashlamp system for two reasons. First, the light pulse generated by the laser had favorable characteristics - a sharp risetime of less than one nanosecond, and an overall width of $\sim 3$ ns. This is to be compared to a width of $300 \mathrm{~ns}$ for the flashlamp pulse. Because of this large width, the use of the Xenon flashlamp system required a special gate in order to use the 

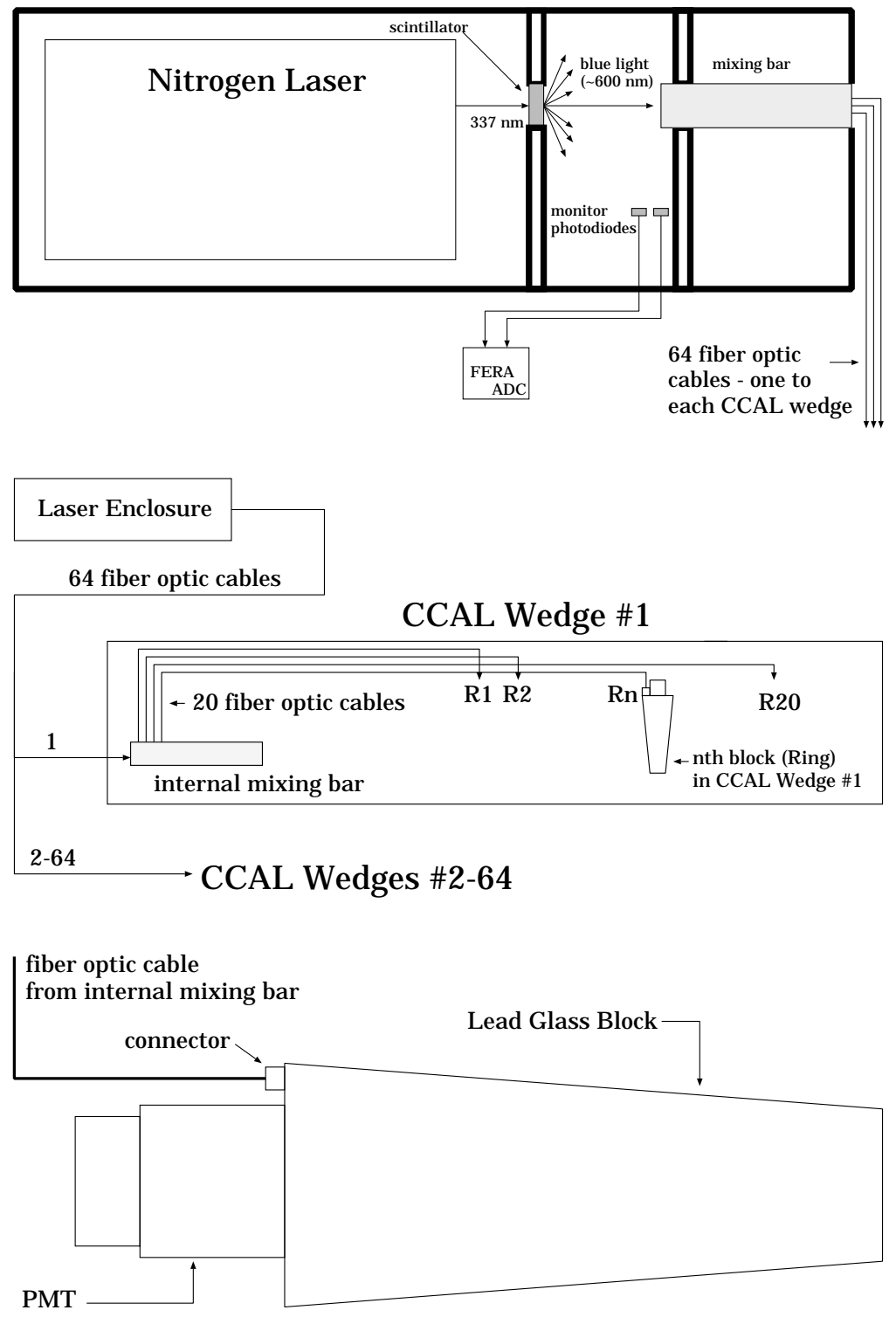

Figure 4.23: Schematics of (top) the CCAL laser enclosure, (middle) the CCAL laser gain monitoring system, showing the distribution of the light from the laser to the 64 CCAL wedges, and (bottom) a typical CCAL lead glass block, showing the attachment of the fiber optic cable, through which laser pulses are transmitted. 
signal for monitoring purposes. For E835, we wished to use the same gate as used for data taking, and this favors the laser option. The $\sim 3$ ns width of the laser pulses resulted in a $\sim 40 \mathrm{~ns}$ wide $(\mathrm{FWHM})$ pulse as measured at the back of the CCAL wedge, essentially the same as pulses due to electrons and photons from real events. (See Fig. 4.24) Also, the laser had good pulse-to-pulse consistency ( 4\%).

We tested the laser system fully before the run, and the 64 external fibers were matched to the 64 wedges so that the light transmitted to each wedge (estimated by measuring the pulse height produced in each block in a wedge, and then comparing wedge averages) was approximately equal, to within $\pm 5 \%$. A special trigger was set up for the laser monitor (see Sec. 5.6) which was pulsed continuously at $0.1 \mathrm{~Hz}$ for the duration of the E835 running.

The CCAL performance ( $\phi, \theta$, and $E$ resolution), the calibration of the CCAL, and the use of these laser monitor data are discussed in Appendix B.

\subsubsection{Luminosity Monitor}

In order to take full advantage of the excellent beam energy resolution afforded by the Antiproton Accumulator, it is necessary that luminosities be measured with an accuracy of a few percent. This is possible with the E835 Luminosity Monitor (LM), built and designed by our Northwestern research group. A full description of the E760 version of the LM is found in Ref. [41].

It was discovered during the course of E760 that the $\bar{p}$ beam had occasionally horizontal displacements which were much larger than those anticipated. In order to monitor such displacements continually, the E835 luminosity monitor was modified. 

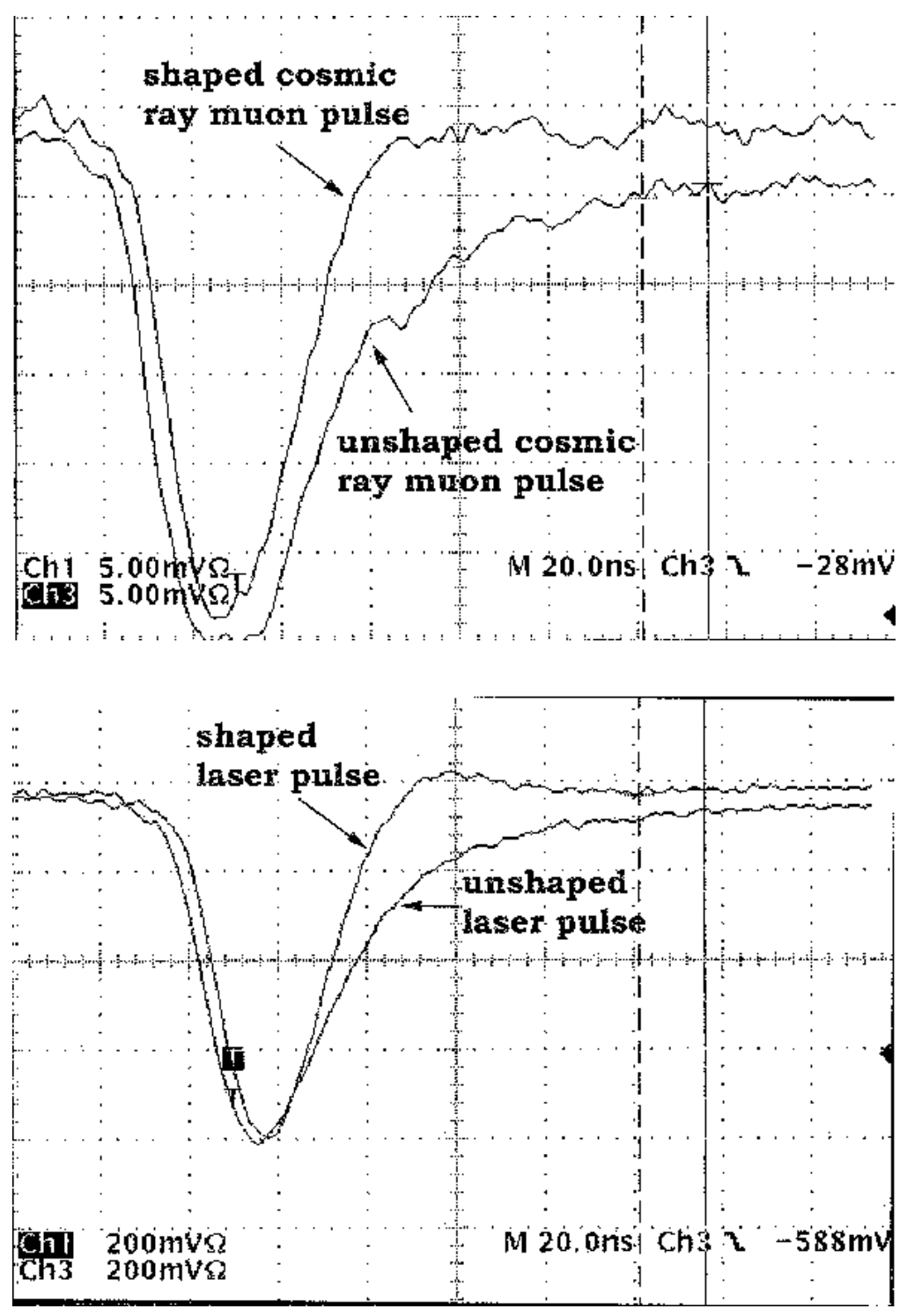

Figure 4.24: Comparison of the signal measured at the end of the CCAL cable harness for pulses due to (top) a cosmic ray muon and (bottom) a pulse from the laser system. The shape of the laser and cosmic signals are similar enough for the laser system to be used as a monitor for gross changes in CCAL channel gain. 
The E835 LM (see Figs. 4.25 and 4.26) therefore consists of three solid state detectors mounted in a steel vacuum vessel below the interaction point, at a polar angle of $\theta_{r} \approx 86.5^{\circ}$. One of these detectors lies directly beneath the beam axis, while the other two lie symmetrically on either side. By comparing relative rates in the "beamleft" and "beam-right" detectors, it is possible to determine whether the $\bar{p}$ beam is displaced with respect to the nominal axis.

The solid angles of the detectors were precisely defined by a machined tungsten mask, with rectangular openings, with each dimension known to \pm 0.0001 inch. The masks for the three detectors had dimensions

$$
\begin{aligned}
(\text { beam left }): 0.3886^{\prime \prime} & \times 0.7889^{\prime \prime} \\
(\text { central }): 0.2752^{\prime \prime} & \times 1.7713^{\prime \prime} \\
(\text { beam right }): 0.3884^{\prime \prime} & \times 0.7886^{\prime \prime}
\end{aligned}
$$

The detector areas were thus known to better than $0.04 \%$. The mask specifications were confirmed with measurements using an $\alpha$ source. The three detectors provided not only for threefold redundancy in luminosity monitoring to safeguard against detector failure, but they also allow precise and continuous monitoring of horizontal displacements of the $\bar{p}$ beam by a comparison of the event rates in the three detectors.

The LM detectors measure proton recoils from low momentum transfer $(t \approx$ 0.0077 to $\left.0.0106(\mathrm{GeV} / \mathrm{c})^{2}\right)$ elastic scattering events. The elastic scattering differential cross sections were measured with better than $\pm 2.5 \%$ precision in E760. [42, 43] 


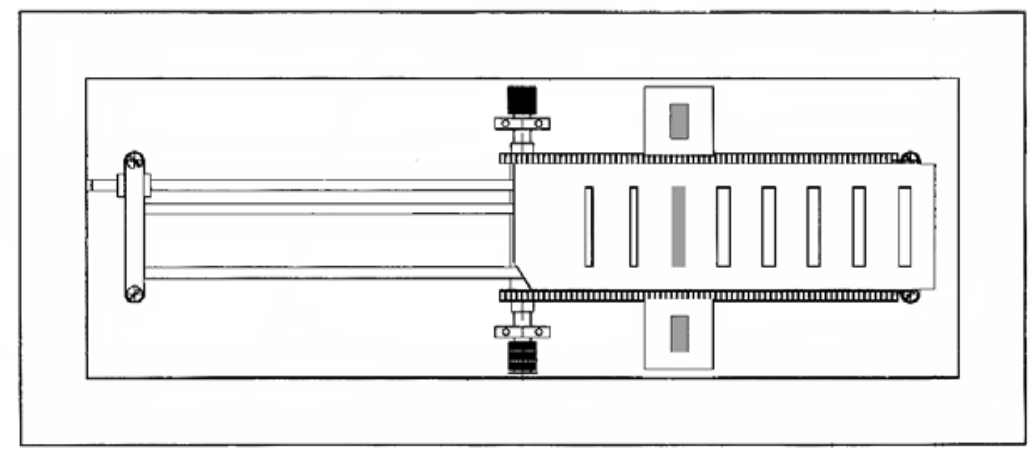

Figure 4.25: Schematic of the E835 Luminosity Monitor detector pan. The active detectors in E835 are indicated by the shading.

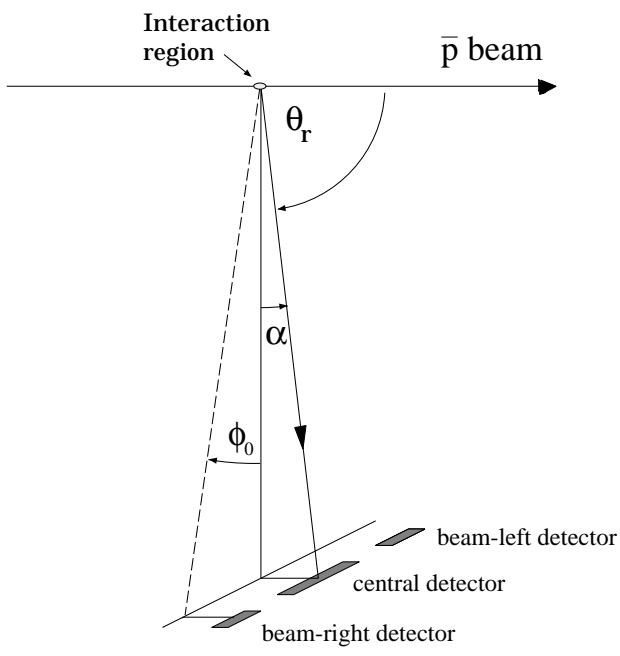

Figure 4.26: Schematic of the E835 Luminosity Monitor showing the three detectors used for monitoring. $\theta_{r}=90^{\circ}-\alpha$ is the proton recoil angle for all three detectors. $\phi$ is the azimuthal angle whose mean value for the three detectors is $-\phi_{0}, 0,+\phi_{0}$. 
By using these we obtain absolute luminosity,

$$
\mathcal{L}=\frac{N}{(d \sigma / d t)(d t / d \Omega)(d \Omega)}
$$

where $\mathrm{N}$ is the number of proton recoils counted, $(d \sigma / d t)$ is the known differential cross section $[42,43]$ and $d \Omega$ is the solid angle subtended by the detector. Examples of proton recoil spectra obtained by one of the LM detectors near the beginning, middle and end of the E835 run are shown in Fig. 4.27. It is to be noted that the detector response was highly stable throughout the run. The signal to noise ratio was typically 30:1. Statistical error in a typical luminosity measurement was very small - the dominant source of error was the error in the differential cross sections, which was estimated to be $\leq \pm 2.5 \%$. [43] 


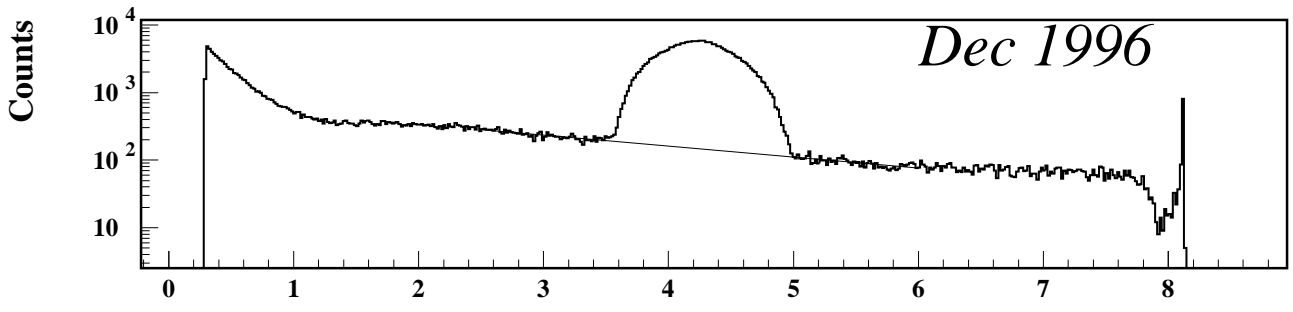

Energy (MeV)

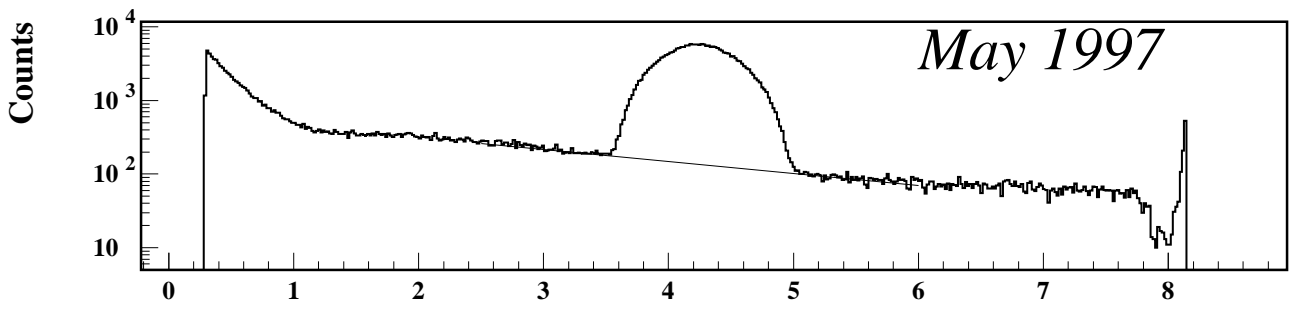

Energy (MeV)

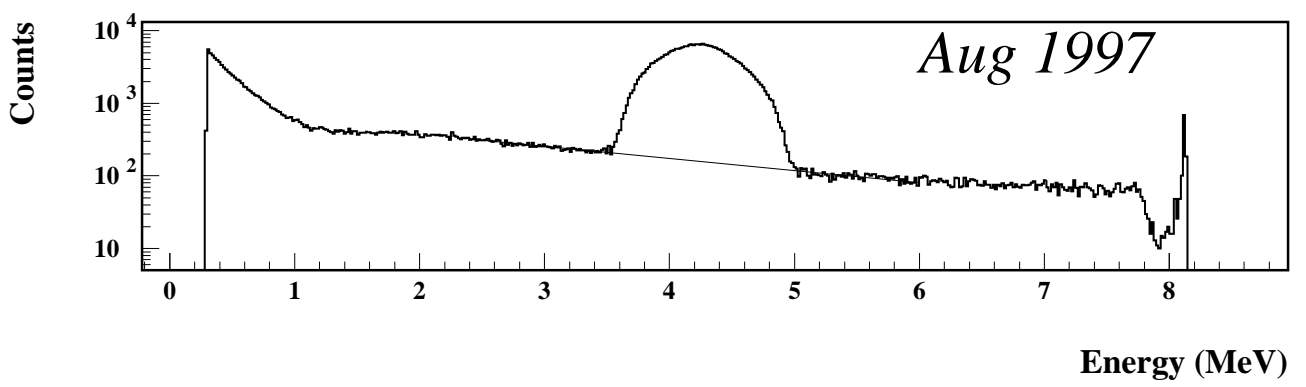

Figure 4.27: Examples of proton recoil spectra obtained with the central detector of the E835 Luminosity Monitor, for data taken in three different running periods. The stability of the detector throughout the E835 run is apparent. 


\section{Chapter 5}

\section{Data Acquisition}

In this chapter we describe acquisition of data in Experiment E835. We include in this discussion descriptions of the hardware with which data from the various detectors are collected, the hardware triggers which define data streams in which events are recorded, and the software triggers (online analysis) which allocate events in each stream into various subsets based on physics criteria. A schematic of the DAQ is shown in Fig. 5.1.

\subsection{DAQ Hardware}

Signals from all detectors are read out by CAMAC modules, both FERA ADCs (typically, LeCroy 4300) and TDCs (LeCroy 3377) which in total fill 19 CAMAC crates. The CAMAC crates are addressed by the run control computer, an SGI Indigo, through two SCSI Jorway Branches (interfaces). Analog signals from each 


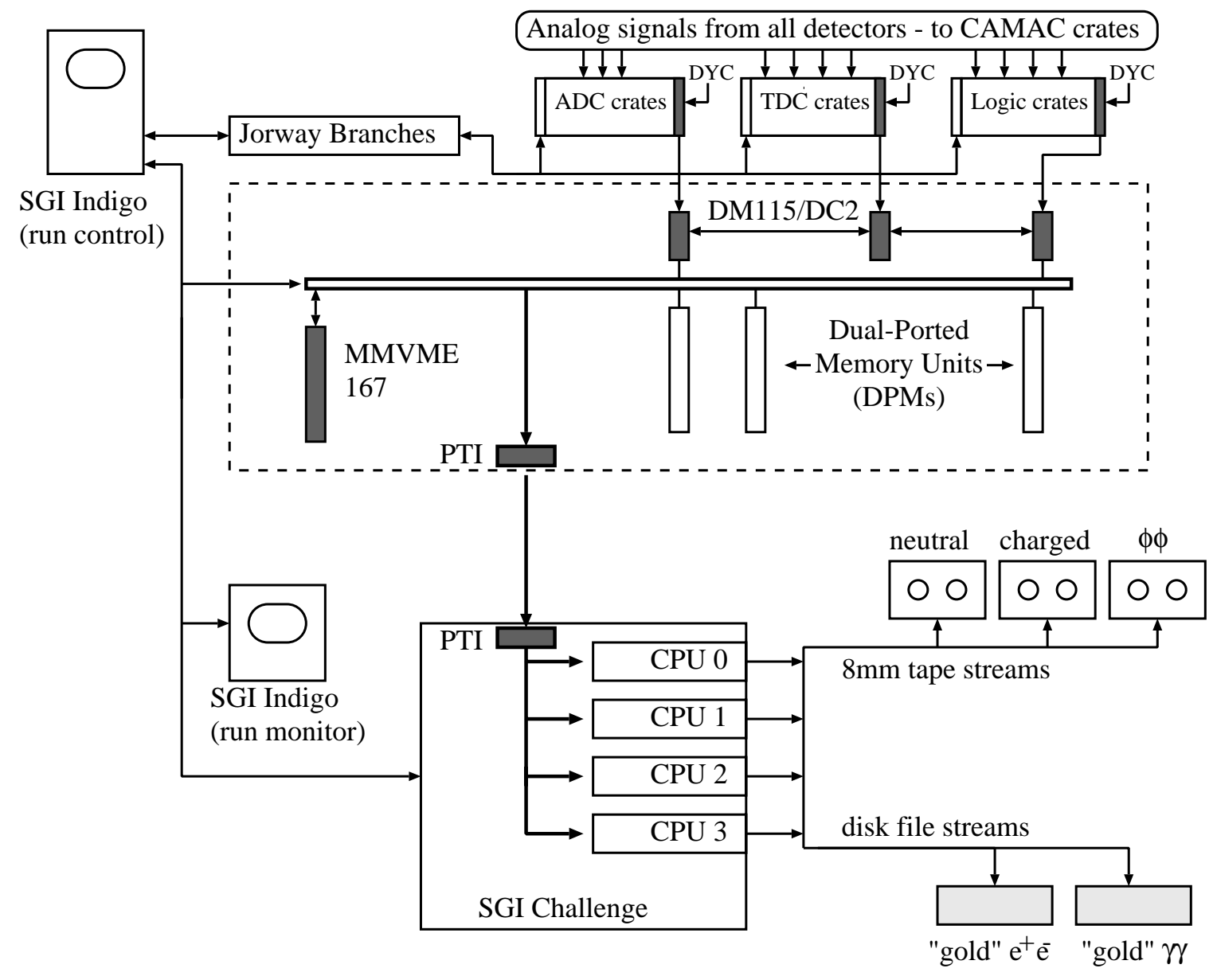

Figure 5.1: Schematic of the E835 Data Acquisition System. 
detector channel are sent to ADCs and TDCs as appropriate, as well as to the logic modules. The data from 14 of the CAMAC crates (those containing exclusively FERA ADCs and TDCs) are read out by custom-made controllers known as DYC+ (Damn Yankee Controllers). The data from the remaining five crates, which contain logic modules with which triggers are constructed, were transferred to the rest of the DAQ system by standard LeCroy CAMAC crate controllers.

The DYC+ crate controllers are able to buffer events in internal FIFOs (FirstIn-First-Out) before sending them via two pairs of Access Dynamics DC2/DM115 units, which transfer the events they receives to several Dual Ported Memory units (DPMs) housed in a VME crate along with a Motorola MMVME167 processor. The MMVME167 then builds events using the data read from the buffer DPMs according to the trigger information received from the Gatemaster (see Sec. 5.7).

Events are recorded by an SGI Challenge workstation with four $150 \mathrm{MHz}$ CPUs. It reads buffered events from the DPMs does some quick online analysis in order to assign events to the various trigger subsets, and sends them according to the encoded trigger information to one of three 8-mm Exabyte tapes and/or to disk. Three Exabyte-8500 tape units were used for data logging, one for each of the three data streams (neutral, charged, and $\phi \phi$ ).

\subsection{E835 Trigger Scheme}

In E835, three basic classes of final states were investigated: neutral, such as $\bar{p} p \rightarrow \gamma \gamma$, $\bar{p} p \rightarrow \pi^{0} \pi^{0}$ or $\bar{p} p \rightarrow \eta \eta \pi^{0}$; charged, such as $\bar{p} p \rightarrow J / \psi \rightarrow e^{+} e^{-}, \bar{p} p \rightarrow J / \psi \gamma \rightarrow e^{+} e^{-} \gamma$ or 
$\bar{p} p \rightarrow \bar{p} p$; and $\phi \phi: \quad \bar{p} p \rightarrow \phi \phi \rightarrow 4 K$. Each of these has a unique event topology and can be selected using a simple set of trigger requirements. The trigger scheme implemented in E835 involved both hardware and software triggers; both are described here. The first and second levels of trigger were hardware triggers, while the third trigger level used the second level hardware triggers and performed fast online event reconstruction to make final trigger assignments.

The hardware triggers are formed by four Memory Lookup Units (MLUs). Each of these has 16 input channels, and performs various logical operations on the inputs to form 8 outputs. The MLU's used in E835 are the charged MLU (CMLU), neutral MLU (NMLU), $\phi \phi$ MLU (PMLU) and the Master MLU (MMLU). The NMLU, CMLU and PMLU* form the Level 1 hardware triggers. Several of these serve as inputs to the MMLU, which constructs logical combinations of them to construct the Level 2 hardware triggers, which in turn are used by the PRUDE filtering software to form the final software triggers (Level 3). The inputs and outputs for the Charged, Neutral and Master MLUs are described below. A schematic illustrating the general trigger layout is presented in Fig. 5.2.

\subsection{The Charged Trigger}

Simple "hit maps" of each charged detector are the primary inputs to the CMLU. Charged Level 1 triggers are constructed by the CMLU using logical combinations of the various CMLU inputs.

${ }^{*}$ The PMLU is relevant only for the $\phi \phi$ and $\bar{p} p$ triggers, which are not used for the analysis presented in this dissertation. 


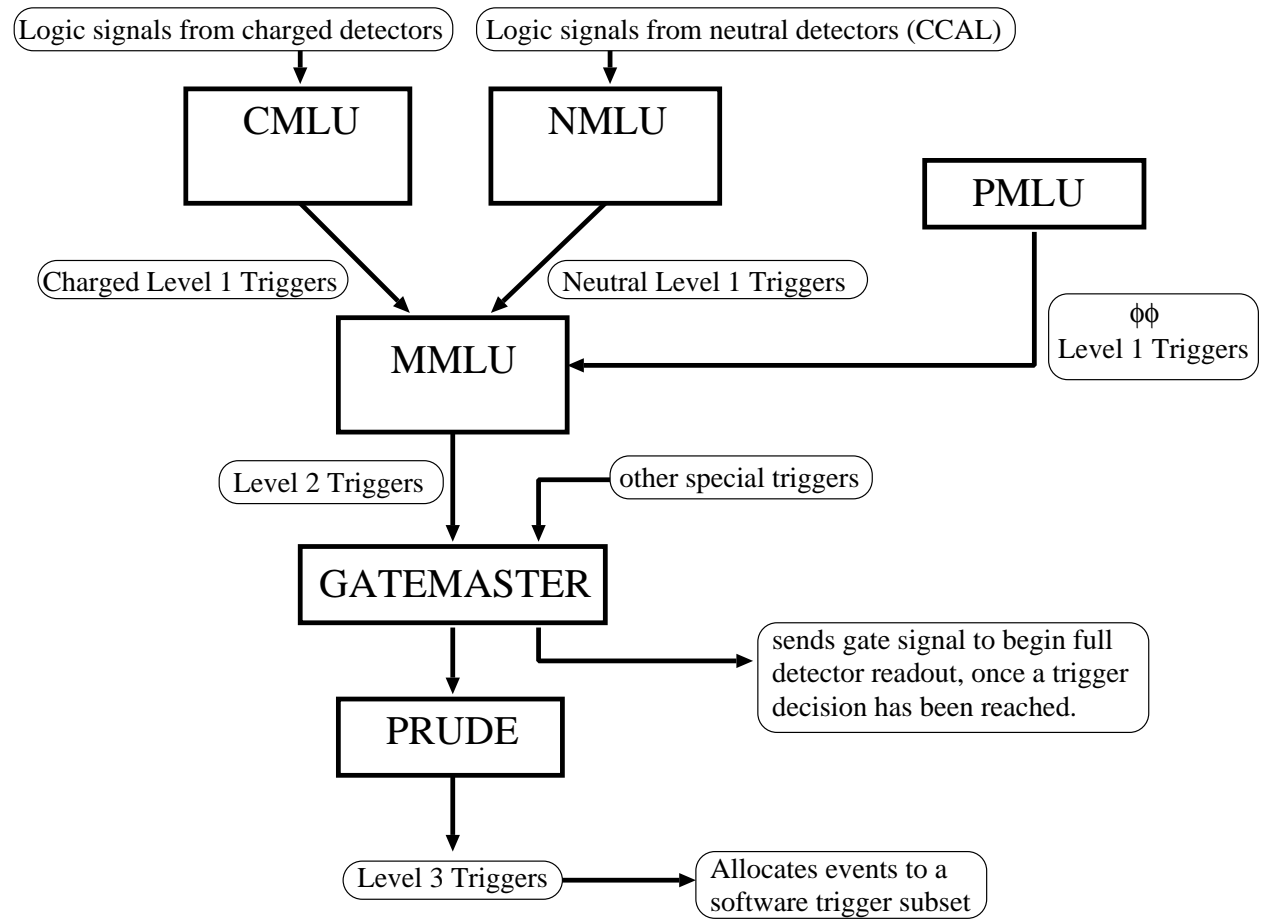

Figure 5.2: Schematic showing the process of E835 trigger construction. 
Table 5.1: Charged MLU inputs and outputs.

\begin{tabular}{ll|ll}
\hline $\begin{array}{l}\text { Input } \\
\text { Channel }\end{array}$ & Description & $\begin{array}{l}\text { Input } \\
\text { Channel }\end{array}$ & Description \\
\hline 1 & 1e: single electron & 9 & H1>4 \\
2 & 2e: double electron & 10 & COPL: H2 coplanarity \\
3 & 1h: single hadron & 11 & FCH-OR \\
4 & 2h: double hadron & 12 & FCAL-OR \\
5 & $H 2=2$ & 13 & H1-OR \\
6 & $H 2>2$ & 14 & H2-OR \\
7 & $H 2>4$ & 15 & empty \\
8 & $H 1>2$ & 16 & empty \\
\hline \hline Output & Description & Output & Description \\
Channel & \multicolumn{3}{l}{ Channel } \\
\hline 1 & $(2 \mathrm{e}) \oplus(\overline{H 2}>4)+(1 \mathrm{e}) \oplus(2 \mathrm{~h}) \oplus(H 2=2) \oplus(\mathrm{COPL})$ \\
2 & $(2 \mathrm{e}) \oplus(H 2=2) \oplus(\mathrm{COPL}) \oplus(\overline{F C H})$ \\
3 & $(2 \mathrm{~h}) \oplus(\mathrm{COPL}) \oplus(\overline{F C H}) \oplus(\overline{F C A L})$ \\
4 & $(2 \mathrm{~h}) \oplus(H 2=2) \oplus(\mathrm{COPL}) \oplus(\overline{F C H}) \oplus(\overline{F C A L})$ \\
5 & $(\overline{1 e}) \oplus(2 \mathrm{~h}) \oplus(H 2=2) \oplus(\mathrm{COPL})$ \\
$6-8$ & empty & \\
\hline
\end{tabular}

\subsubsection{Charged MLU}

The inputs and outputs of the CMLU are summarized in Fig. 5.3 and Table 5.1, and are briefly described below. 


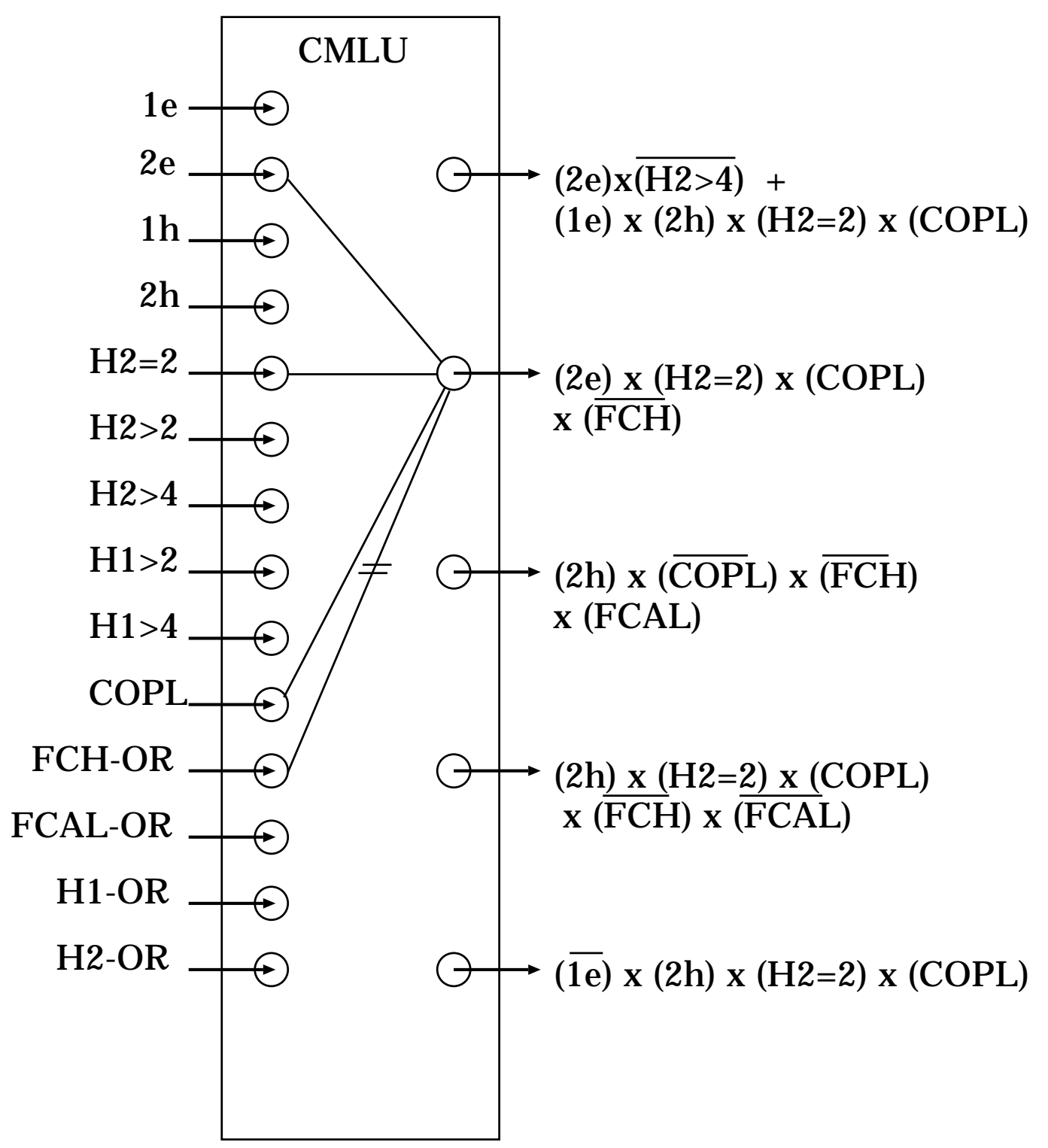

Figure 5.3: Charged MLU inputs and outputs. The lines indicate the logical construction of CMLU2 from CMLU inputs 2, 5, 10 and 11. 


\section{CMLU Inputs}

1. Single Electron Tag (1e): ${ }^{\dagger}$ Requires a single hadron tag and a hit in one or both of the corresponding Čerenkov cells (forward and backward).

2. Double Electron Tag (2e): Two single electron tags in coincidence.

3. Single Hadron Tag (1h): A coincidence between an $H 1$ element and one of the corresponding $6 \mathrm{H} 2$ elements.

4. Double Hadron Tag (2h): 2 hadron tags in coincidence.

5. Hodoscope multiplicity, $H 2=2$ : Exactly $2 H 2$ paddles hit.

6. Hodoscope multiplicity, $H 2>2$ : More than $2 H 2$ paddles hit.

7. Hodoscope multiplicity, $H 2>4$ : More than $4 H 2$ paddles hit.

8. Hodoscope multiplicity, $H 1>2$ : More than $2 H 1$ paddles hit.

9. Hodoscope multiplicity, $H 1>4$ : More than $4 H 1$ paddles hit.

10. Coplanarity (COPL): There is a $2 \mathrm{~h}$ tag (CMLU input \#2) or 2e tag (CMLU input \#4) involving $H 2$ elements which are roughly back-to-back in azimuth. (i.e., separated by 15, 16 or 17 elements, out of the total of 32)

11. Forward Charged OR (FCH-OR): There is a hit in any FCH element.

\footnotetext{
${ }^{\dagger}$ I disavow any responsibility for the choice of nomenclature in the charged trigger, evident here, wherein an electron begins its life as a hadron. Neither will I comment on the choice of placing the $1 \mathrm{e}$ and $2 \mathrm{e}$ tags in positions 1 and 2, while simpler trigger inputs ( $1 \mathrm{~h}$ and $2 \mathrm{~h}$ ) are in positions 3 and 4.
} 
12. Forward Calorimeter OR (FCAL-OR): There is a hit in any FCAL element.

13. $H 1 \mathrm{OR}(\mathrm{H} 1 \mathrm{OR})$ : There is a hit in any $H 1$ element.

14. H2 OR (H2OR): There is a hit in any H2 element.

15.-16. Empty.

\section{CMLU Outputs: Level 1 triggers}

Each of the CMLU outputs is a logical combination of several of the inputs; these are then used as input to the Master MLU, from which the Level 2 triggers are formed. CMLU outputs 1 and 2 are used in the formation of the $e^{+} e^{-}$trigger; CMLU 3 is part of the $\phi \phi$ trigger; and CMLU4 is used in the $\bar{p} p$ trigger. CMLU5 was ultimately not used.

\subsection{The Neutral Trigger}

The neutral trigger is constructed using signals from the CCAL alone, since it is the only detector sensitive to neutral particles. The hardware for the E835 neutral trigger is shown schematically in Fig. 5.4. The neutral MLU (NMLU) produces four Level 1 triggers: triggers for two-body events, called PBG1 and PBG3, and triggers

for multi-photon events $\left(\pi^{0} \pi^{0}, \pi^{0} \eta\right.$, etc), called ETOT-HI and ETOT-LO. A full description of the neutral trigger used in E760 is found in Ref. [44], and updates for E835 are discussed in Ref. [45]. 


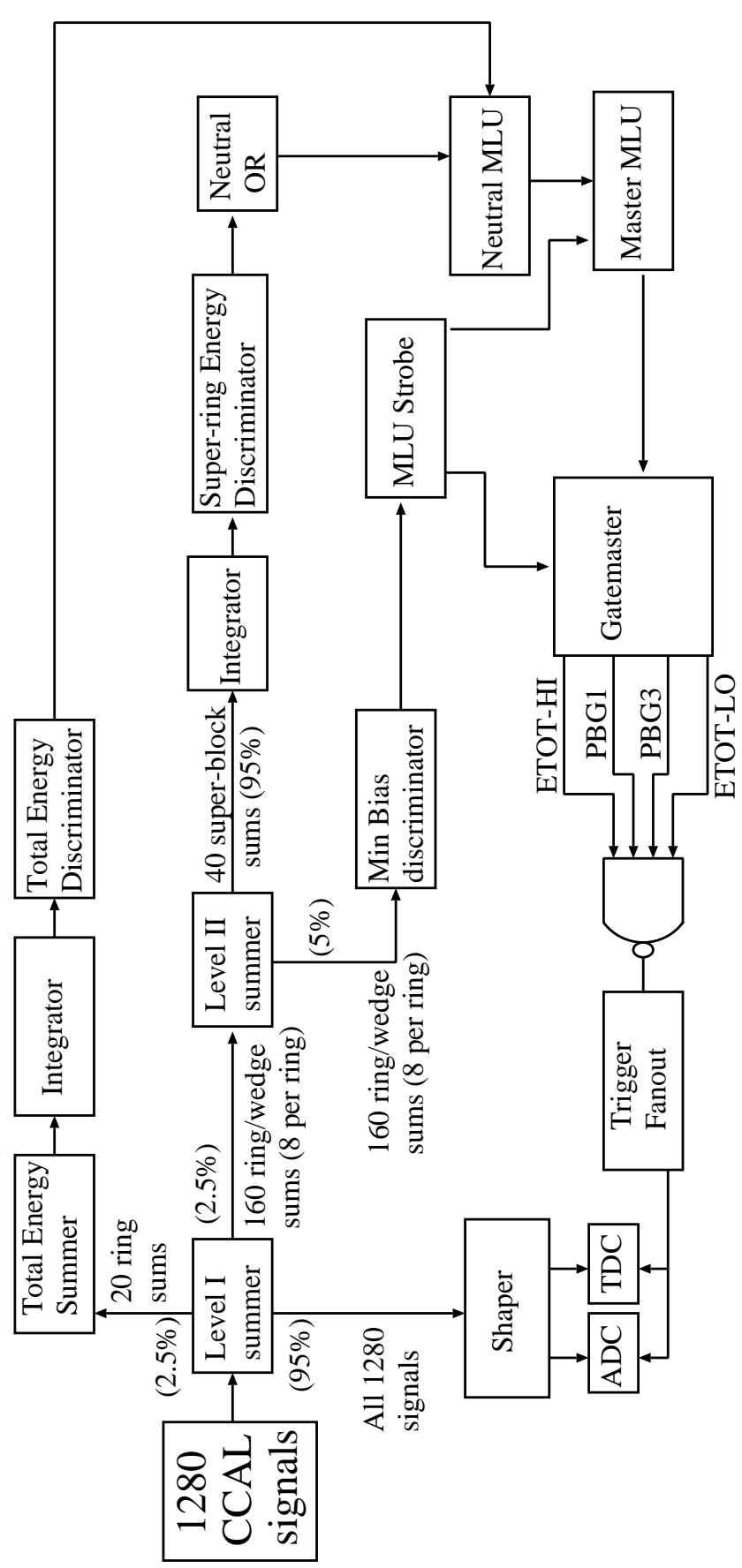

Figure 5.4: Schematic of the E835 Neutral Trigger. The numbers in parentheses denote the fraction of the signal input to each summer which is used for the indicated operation. For example, 2.5\% of the output from the Level I summers is used as input (after amplification by a factor 20) to the Level II summers. 
Table 5.2: Super-blocks and their constituents.

\begin{tabular}{|l|l|}
\hline Super-wedge & CCAL wedges \\
\hline 1 & $1-9$ \\
2 & $9-17$ \\
3 & $17-25$ \\
4 & $25-33$ \\
5 & $33-41$ \\
6 & $41-49$ \\
7 & $49-57$ \\
8 & $57-1$ \\
\hline
\end{tabular}

\begin{tabular}{|l|l|}
\hline Super-ring & CCAL ring \\
\hline 1 & $1-4$ \\
2 & $4-8$ \\
3 & $8-12$ \\
4 & $12-16$ \\
5 & $16-20$ \\
\hline
\end{tabular}

In order to reduce the number of inputs to the NMLU, the signals from the 1280 blocks of the CCAL are summed to produce 40 signals which can be used to create triggers based on event topology. The 20 rings of the CCAL are summed to create 5 "super-rings", and the 64 wedges are summed to create 8 "super-wedges" the resulting 40 super-ring/super-wedge combinations are known as "super-blocks". The pattern of hits in these super-blocks determines which, if any, neutral trigger an event satisfies.

A "roll-out" diagram of the CCAL, showing the allocation of rings and wedges to the super-rings and super-wedges discussed in this section, is presented in Fig. 5.5. In Table 5.2 we list the rings and wedges which contribute to each super-ring and superwedge sum. Each super-ring overlaps the adjacent super-ring by one ring; similarly, each super-wedge overlaps the adjacent super-wedge by one wedge. (See Fig. 5.5) The overlaps are necessary to avoid trigger inefficiencies due to a particle dividing its energy between two neighboring super-blocks. 


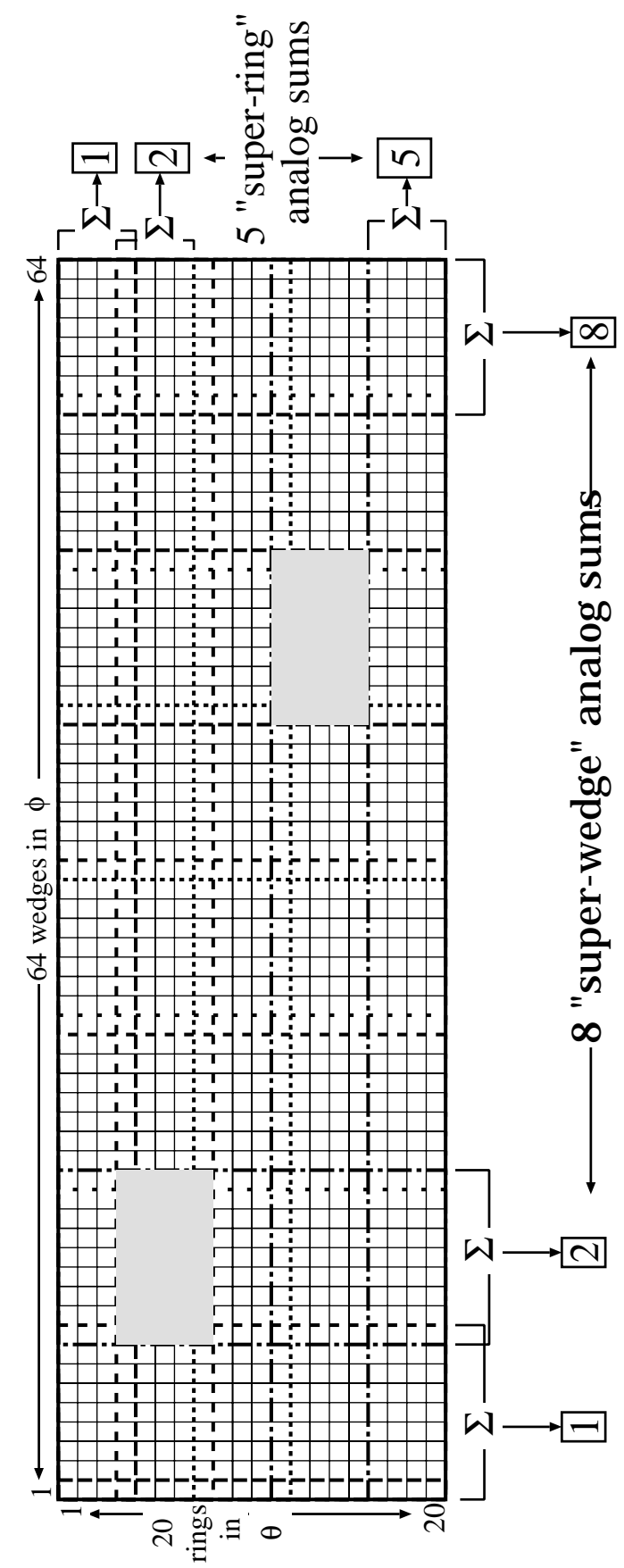

Figure 5.5: Rollout diagram of the 1280 CCAL elements. Super-ring and Superwedge boundaries are indicated by the heavy dashed lines. Indicated are hypothetical hits in SR,SW $=2,2$ and 4,6. 


\subsubsection{Level I Summers}

There are 20 identical Level I summer modules, which each make analog sums of the 64 blocks in a CCAL ring. $5 \%$ of the signal from each CCAL block is split off and used for summing. The other $95 \%$ is sent from the Level I summer (in the pit) up to the Shaper circuit boards (see Sec. 4.4.3) in the counting room.

Within each Level I summer module, two summing operations take place. First, $2.5 \%$ of the CCAL signal is used to make a sum over all 64 blocks in the ring. Then the ring sum is transported upstairs to a Total Energy summer, for use in the ETOT triggers. Second, eight analog sums (each of which represents part of a super-wedge sum) are formed using the remaining $2.5 \%$ of the signal. Each of these sums is formed from the signals from nine adjacent blocks in the ring, with one block overlap.

The 160 Level I summer signals $(20$ summers $\times$ eight super-wedge sums from each) are transported upstairs from the pit to the eight Level II summer modules in the counting room. At the input to the Level II summer module, the signals are amplified by a factor of 20 .

\subsubsection{Level II Summers}

Each of the eight Level II summer modules, which corresponds to a particular superwedge, takes as input from each Level I summer one of the eight "partial superwedge sums" described above. At the input of the Level II module the signals from the Level I summers are amplified by a factor of $20.5 \%$ of each of these 20 input signals is passed directly through the Level II summer for use in the MLU strobe. 
Table 5.3: Weights for each input to the Level II summers, according to CCAL ring. Rings 4, 8, 12 and 16 have two entries, as the signals from each are sent to two super-rings.

\begin{tabular}{cc|ll|lll|ll|lc}
\hline \multicolumn{2}{c}{ Super-ring 1 } & \multicolumn{2}{c}{ Super-ring 2 } & \multicolumn{2}{c}{ Super-ring 3 } & \multicolumn{2}{c}{ Super-ring 4 } & \multicolumn{2}{c}{ Super-ring 5 } \\
\hline \multicolumn{2}{c}{ Ring } & weight & Ring & weight & Ring & weight & Ring & weight & Ring & weight \\
\hline & & 4 & 1.21 & 8 & 1.19 & 12 & 1.10 & 16 & 1.05 \\
2 & 1.10 & 5 & 1.10 & 9 & 1.07 & 13 & 1.05 & 17 & 1.02 \\
3 & 1.00 & 6 & 1.00 & 10 & 1.00 & 14 & 1.00 & 18 & 1.00 \\
4 & 0.91 & 7 & 0.91 & 11 & 0.95 & 15 & 0.95 & 19 & 0.98 \\
\hline
\end{tabular}

(see Sec. 5.7) The other $95 \%$ is weighted (see Table 5.3) and summed to form five "super-rings", with one ring overlapping the adjacent super-ring.

The weighting of the ring sums allows for a more efficient trigger based on twobody thresholds. This is illustrated in Fig. 5.6, which shows the results of a Monte Carlo simulation of $J / \psi \rightarrow e^{+} e^{-}$events with and without weighting of ring sums. [44] Because of the weighting, a clear energy threshold for each super-ring may be set for two-body kinematics.

The output from each of the eight Level II summers consists of 5 "super-block" signals, one for each super-ring within the superwedge to which that Level II summer corresponds. Each of the 40 super-block signals, weighted as mentioned above, are passed to a discriminator, whose level is set at $\sim 60 \%$ of the energy expected for one of the daughters of a two-body decay which strikes that super-block. The resulting 40 logic signals are used to build the PBG1 and PBG3 triggers as discussed in the next section. 

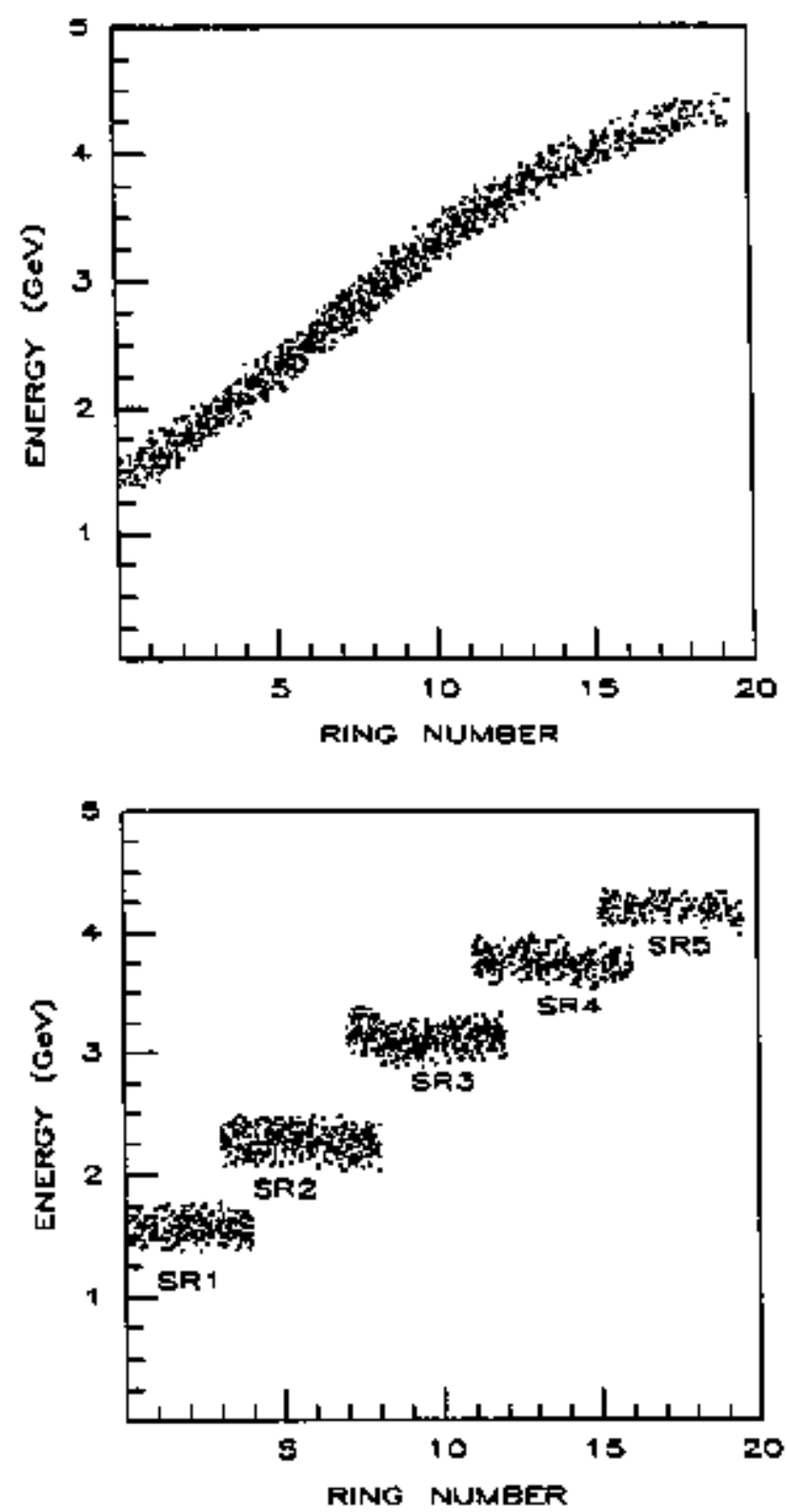

Figure 5.6: Results of an E760 Monte Carlo simulation of the reaction $\bar{p} p \rightarrow J / \psi \rightarrow e^{+} e^{-}$. The energy of both electrons in each event is plotted, as a function of its polar angle (in units of CCAL ring). In the top plot, the raw energies are shown, and in the bottom plot, these energies have been multiplied by the weighting factor for each ring, to show the super-ring separation which is achieved in the Level II summers. Two-body thresholds are thus easily set. 
Table 5.4: Neutral MLU inputs and outputs.

\begin{tabular}{ll|ll||ll}
\hline $\begin{array}{l}\text { Input } \\
\text { Channel }\end{array}$ & Description & $\begin{array}{l}\text { Input } \\
\text { Channel }\end{array}$ & Description & $\begin{array}{l}\text { Output } \\
\text { Channel }\end{array}$ & Description \\
\hline 1 & Superwedge 1 & 9 & ETOT-HI & 1 & PBG1 \\
2 & Superwedge 2 & 10 & ETOT-LO & 2 & PBG3 \\
3 & Superwedge 3 & 11 & Empty & 3 & ETOT-HI \\
4 & Superwedge 4 & 12 & Empty & 4 & ETOT-LO \\
5 & Superwedge 5 & 13 & Empty & 5 & Empty \\
6 & Superwedge 6 & 14 & Empty & 6 & Empty \\
7 & Superwedge 7 & 15 & Empty & 7 & Empty \\
8 & Superwedge 8 & 16 & Empty & 8 & Empty \\
\hline
\end{tabular}

\subsubsection{Neutral MLU}

The neutral MLU inputs and outputs are summarized in Fig. 5.7 and Table 5.4, and we describe them below.

\section{NMLU Inputs}

As shown in Table 5.4 ach of the inputs $1-8$ to the NMLU is a logical OR of the five discriminated super-ring signals in each super-wedge formed by the appropriate Level II summer (1-8). A hit above energy threshold in any of the five super-rings results in the corresponding superwedge NMLU input being "on". The 9th and 10th NMLU inputs are based on the Total Energy sum which is formed from the ring sum outputs from each Level I summer. Input channels 11-16 are presently empty. 


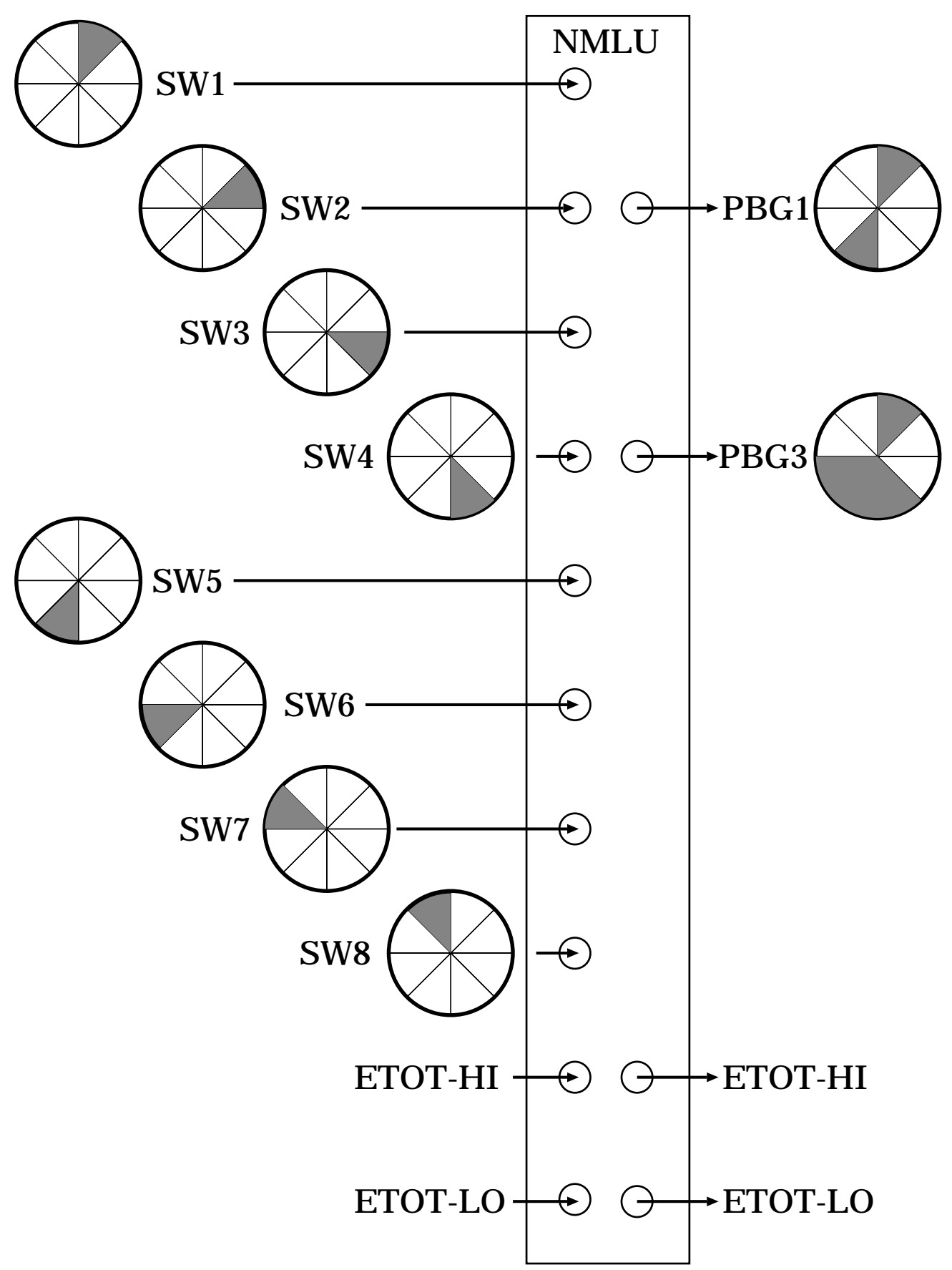

Figure 5.7: Neutral MLU inputs and outputs. The small diagrams illustrate the CCAL requirements for each input and output. For instance, the NMLU input \#1 (SW1) requires a hit above two-body threshold in Superwedge 1 (wedges 1-9). 


\section{NMLU Outputs}

1. PBG1 (CCAL tight 2-body): This requires that the inputs corresponding to two back-to-back superwedges be "on"; i.e., this requires hits in two superblocks consistent with two-body kinematics.

2. PBG3 (CCAL loose 2-body): Similar to PBG1, except that PBG3 makes a looser "back-to-back" requirement - a hit in one superwedge must be accompanied by one in the opposing three. This trigger is designed for the $e^{+} e^{-} \gamma$ final state, in which the $e^{+}$and $e^{-}$may be significantly out of coplanarity due to the large recoil $\gamma$.

3. Total Energy HI (ETOT-HI): The sum of all blocks in CCAL was greater than $80 \%$ of the total available energy.

4. Total Energy LO (ETOT-LO): The sum of all blocks in CCAL was greater than $70 \%$ of the total available energy.

\subsection{Master MLU}

As stated in Sec. 5.2, the outputs from the charged, neutral and $\phi \phi$ MLUs form the first level hardware triggers. The Master MLU takes these Level 1 triggers as inputs and constructs Level 2 triggers as described below. 


\section{MMLU Inputs}

The master MLU takes 16 inputs: NMLU outputs 1-4 (constituting inputs 1-4), four logical detector combinations used to construct vetoes of the neutral trigger (inputs 5-8, three of which are the same as CMLU inputs 6, 11 and 12), five outputs from the CMLU (inputs 9-13) and three outputs from the PMLU (inputs 14-16). As the name suggests, the MMLU is the source of the Master trigger. Its inputs and outputs are summarized in Table 5.5.

Input 5 is the only MMLU input which is not simply an input or output from the other MLUs. It is ON if there is any hit in $\mathrm{H} 1$ and a hit in one of the four corresponding elements of $H 2^{\prime}$. The Neutral Veto trigger bit is composed of this $\left(H 1 \cdot H 2^{\prime}-O R\right)$ and Input 8 (FCH-OR).

\section{MMLU Outputs}

The outputs from the MMLU constitute the Level 2 triggers.

- MMLU1 is the logical OR of three MMLU inputs, which together form the $e^{+} e^{-}$trigger. The first, $(2 \mathrm{e}) \oplus(\overline{H 2>4}) \oplus \mathrm{PBG} 3$ is known as the "2CัC" trigger, since it requires the most stringent conditions - 2 electrons, back to back. The second, $(1 \mathrm{e}) \oplus(2 \mathrm{~h}) \oplus(H 2=2) \oplus(\mathrm{COPL}) \oplus \mathrm{PBG} 3$, is called the "1C̆" trigger and allows for inefficiencies in the Čerenkov by requiring only one "electron" track, while requiring specifically 2 tracks through the hodoscopes $H 1$ and H2. The third is the "NO-CCAL" trigger. It requires the tightest cuts on the Cerenkov and $H 2$, but makes no requirement on the CCAL. It is a test trigger used to evaluate the efficiencies of PBG1 and PBG3. 
Table 5.5: Master MLU inputs and outputs.

\begin{tabular}{|c|c|c|}
\hline $\begin{array}{l}\text { Input } \\
\text { Channel }\end{array}$ & Description & $\begin{array}{ll}\text { Input } & \text { Description } \\
\text { Channel } & \\
\end{array}$ \\
\hline 1 & PBG1 & CMLU 1 \\
\hline 2 & PBG3 & CMLU 2 \\
\hline 3 & ETOT-HI & CMLU 3 \\
\hline 4 & ETOT-LO & CMLU 4 \\
\hline 5 & $\left(H 1 \cdot H 2^{\prime}\right)-\mathrm{OR}$ & CMLU 5 \\
\hline 6 & FCAL-OR & PMLU 1 \\
\hline 7 & $H 2>2$ & PMLU 2 \\
\hline 8 & FCH-OR & PMLU 3 \\
\hline $\begin{array}{l}\text { Output } \\
\text { Channel }\end{array}$ & Description & \\
\hline 1 & \multicolumn{2}{|c|}{$\begin{array}{l}e^{+} e^{-}:(2 \mathrm{e}) \oplus(\overline{H 2>4}) \oplus \mathrm{PBG} 3 \\
+(1 \mathrm{e}) \oplus(2 \mathrm{~h}) \oplus(H 2=2) \oplus(\mathrm{COPL}) \oplus \mathrm{PBG} 3 \\
+(2 \mathrm{e}) \oplus(H 2=2) \oplus(\mathrm{COPL}) \oplus(\overline{F C H})\end{array}$} \\
\hline 2 & \multicolumn{2}{|l|}{$\bar{p} p 90^{\circ}$} \\
\hline 3 & \multicolumn{2}{|l|}{$\phi \phi$} \\
\hline 4 & \multicolumn{2}{|c|}{$\gamma \gamma: \mathrm{PBG} 1 \oplus\left(\overline{H 1 \cdot H 2^{\prime}}\right) \oplus(\overline{F C H})$} \\
\hline 5 & \multicolumn{2}{|c|}{$($ ETOT-HI $) \oplus\left(\overline{H 1 \cdot H 2^{\prime}}\right) \oplus(\overline{F C H})$} \\
\hline 6 & \multicolumn{2}{|l|}{$\bar{p} p 55^{\circ}$} \\
\hline 7 & \multicolumn{2}{|c|}{$($ ETOT-HI $) \oplus(\overline{H 2=2})$} \\
\hline 8 & \multicolumn{2}{|c|}{$($ ETOT-LO $) \oplus(\overline{H 2=2}) \oplus(\overline{F C H})$} \\
\hline
\end{tabular}


- MMLU2 is a trigger which uses information from the Scintillating Fiber tracker in order to select $\bar{p} p$ elastic scattering events at $90^{\circ}$ in the $\mathrm{CM}$ frame. This trigger requires the $2 \mathrm{~h}$ tag with $\mathrm{H} 2=2$, COPL, and no FCH or FCAL hits (CMLU output 4) In addition, depending on the CM energy, hits in appropriate scintillating fiber bundles (those which corresponded to $90^{\circ}$ for that particular CM energy) are required.

- MMLU3 is the trigger for $\phi \phi$. Several different configurations were used during the run - all of them attempted to use combinations of hits in the Scintillating Fibers and the Hodoscopes corresponding to four charged tracks, loosely consistent with $\phi \phi \rightarrow 2\left(\mathrm{~K}^{+} \mathrm{K}^{-}\right)$.

- MMLU4 is the $\gamma \gamma$ trigger, which is the most important trigger as far as we are concerned in this dissertation. It makes a hard requirement on the CCAL (PBG1) - two back-to-back super-blocks, and requires the Neutral Veto bit to be OFF.

- MMLU5 is the ETOT-HI w/Neutral Veto trigger. This contains all multi- $\gamma$ final states (such as $\eta \eta \pi^{0}, \eta \pi^{0} \pi^{0}$, etc) which do not satisfy MMLU4.

- MMLU6 is a control trigger for $\bar{p} p \rightarrow \bar{p} p$ at $55^{\circ}$, used to evaluate the $\bar{p} p$ elastic trigger, MMLU2.

- MMLU7 and MMLU8 are triggers used to study efficiencies of various other triggers. 


\subsection{Other Triggers}

There are three additional triggers which are not formed by the MMLU. These are the minimum bias trigger, the laser monitor trigger, and the random gate trigger.

\subsubsection{Minimum Bias}

The minimum bias trigger is used to check various systematics for the experiment. It is formed using the logical OR of the 160 Level I summer outputs from the CCAL; so that if there is energy in the CCAL, undefined as to the pattern, this trigger bit is $\mathrm{ON}$.

\subsubsection{Laser Monitor}

The trigger for the laser monitor is provided by a $0.1 \mathrm{~Hz}$ pulser, which not only gives the trigger but also pulses the laser which in turn illuminates all 1280 blocks of the CCAL. The use of the Laser Monitor data for evaluation of the stability of CCAL PMT gains is described in Appendix B.

\subsubsection{Random Gate}

A very important trigger for the determination of various inefficiencies of other triggers and/or analysis cuts is the random gate trigger. It triggers readout of all the detector elements by means of a $10 \mathrm{kHz}$ pulser. Because the random gate trigger does not depend on the signal in any detector element, unlike the minimum bias 
trigger, it is essentially the no-bias trigger.

\subsection{The Gatemaster}

The CAMAC module which enables readout of the data from the detectors is called the Gatemaster. It provides the gate signal to all the experiment's DYC+ modules (i.e. all the readout electronics). In order for the gate signal to be given, the Gatemaster must receive a strobe. This strobe is provided by the minimum bias trigger so that gates can be started whenever there is energy detected in the CCAL. When a strobe is received by the Gatemaster the gate signal is sent to all the DYC+'s. After each gate signal is sent, the Gatemaster goes into INHIBIT mode for $\sim 10 \mu \mathrm{s}$, in order to allow time for all the detector CAMAC modules to be cleared and be ready for the next event. If a strobe is received by the Gatemaster while in this INHIBIT mode, it is ignored.

The Gatemaster takes 16 inputs, which represent 16 trigger conditions. (See Table 5.6) Eight of these (input channels 1-8) are simply outputs from the Master MLU. The others are additional efficiency-checking and monitoring triggers which were described in the last section. When the Gatemaster receives a strobe, it polls its inputs to see if any of them is ON, i.e. if any hardware trigger conditions have been satisfied. If there is at least one trigger bit ON, then the gates are sent out to the various CAMAC crates, and the software trigger program, PRUDE takes over for the purpose of assigning a more specific software trigger to the event. Each time a strobe is received and a particular hadware trigger is ON, a CAMAC scaler 
Table 5.6: Gatemaster Trigger Inputs: priority list

\begin{tabular}{ll|ll}
\hline GM \# & Trigger description & GM \# & Trigger description \\
\hline 1 & $e^{+} e^{-}$ & 9 & Laser Monitor \\
2 & $\bar{p} p 90^{\circ}$ & 10 & Silicon Strobe \\
3 & $\phi \phi$ & 11 & Empty \\
4 & Neutral PBG1 & 12 & Minimum Bias \\
5 & Neutral ETOT & 13 & Random Gate \\
6 & $\bar{p} p$ control $55^{\circ}$ & 14 & FCAL Cosmic Ray \\
7 & ETOT-HI No Veto & 15 & High Rate Min Bias \\
8 & ETOT-LO Neutral & 16 & Empty \\
\hline
\end{tabular}

channel dedicated to that GM\# is incremented.

Each of the 16 GM inputs also has what is called an autopass number. That is, when the scaler for a particular GM\# reaches its autopass value, PRUDE (the software trigger algorithm) is instructed to pass the event, regardless of any other information, to the appropriate tape file, with an entry made in the data record to indicate that it passed as an autopass event. These autopass-triggered events are used to check efficiencies of the PRUDE filtering.

\subsection{Software Trigger Level: PRUDE}

The software trigger for a given event is determined by an online program called PRUDE - Program for Rejecting Unwanted Data Events. Using the Level 2 trigger information provided by the Gatemaster, PRUDE does some simple online event reconstruction in order to determine whether the event conforms to certain minimum requirements, and should be kept. If the event is to be kept, PRUDE assigns it to 
a more specific trigger subset.

The PRUDE software does fast online event reconstruction using CCAL information. At this stage, PRUDE finds local energy maxima in the CCAL, (blocks containing at least $50 \mathrm{MeV}$, and larger in energy than any of their 8 nearest neighbors) and considers the 8 surrounding blocks around maxima to make up a "cluster". Energy for the 9 blocks is summed, and the angles $\theta$ and $\phi$ are determined by a simple energy-weighted average over the 9 blocks in the cluster. An invariant mass (called the "cluster mass") is also calculated for the cluster. This is a very coarse approximation of the offline clustering analysis which is described later in Sec. 6.1.

This procedure allows for a very fast calculation which can be used on-line to label an event as one of many different PRUDE trigger types. These clusters are used to calculate invariant masses of large energy pairs, and used to find events which include $\pi^{0}$ 's and/or $\eta$ 's. In this section (and this section only) when we speak of "clusters" we are speaking of these on-line PRUDE clusters.

A given event may satisfy several PRUDE trigger conditions. In such a case the event is regarded as satisfying the trigger condition which has the highest priority. The trigger priorities are listed in Table 5.7. The highest priority is assigned to the fifteen autopass triggers, in the order presented in Table 5.7. After these, the priorities are as follows: (in the list below, $\mathcal{M}$ denotes the largest invariant mass combination of two clusters in the event)

\section{Priority Number:}

16. "gold ee" - GM1 events for which $\mathcal{M} \geq 2.0(2.2) \mathrm{GeV}$ below (above) the Accumulator transition energy (approximately $\sqrt{s}=3.4 \mathrm{GeV}$ ). 
Table 5.7: PRUDE trigger priorities.

\begin{tabular}{ll|ll}
\hline Priority & Name & Priority & Name \\
\hline 1 & GM9 autopass & 17 & good ee \\
2 & GM12 autopass & 18 & elec \\
3 & GM13 autopass & 19 & phi-phi \\
4 & GM7 autopass & 20 & gold $\boldsymbol{g g}$ \\
5 & GM14 autopass & 21 & good gg \\
6 & GM8 autopass & 22 & eta invm \\
7 & GM15 autopass & 23 & pi invm \\
8 & GM1 autopass & 24 & cma invm \\
9 & GM4 autopass & 25 & cmb invm \\
10 & GM10 autopass & 26 & invmass \\
11 & GM3 autopass & 27 & eta etot \\
12 & GM2 autopass & 28 & pi etot \\
13 & GM6 autopass & 29 & cma etot \\
14 & GM5 autopass & 30 & cmb etot \\
15 & GM11 autopass & 31 & etot \\
16 & gold ee & 32 & neut \\
\hline
\end{tabular}


17. "good ee" - GM1 events for which $2.2 \geq \mathcal{M} \geq 2.0 \mathrm{GeV}$. Below transition, this trigger is not active.

18. "elec" - GM1 events for which $\mathcal{M}<2.0 \mathrm{GeV}$.

19. "phi-phi" - GM3 events with further cuts on kinematics and scintillating fiber combinations.

20. "gold gg" - GM4 or GM5 events for which $\mathcal{M} \geq 2.5(2.7) \mathrm{GeV}$ below (above) transition.

21. "good gg" - GM4 or GM5 events for which $2.7 \geq \mathcal{M} \geq 2.5 \mathrm{GeV}$. Below transition, as for "good ee", this trigger was inactive.

22. "eta invm" - GM4 or GM5 events which include at least one exclusive $\eta,<6$ CCAL clusters, and $\mathcal{M} \geq 2.0 \mathrm{GeV}$.

23. "pi invm" - GM4 or GM5 events which include at least one exclusive $\pi^{0},<6$ CCAL clusters, and $\mathcal{M} \geq 2.0 \mathrm{GeV}$.

24. "cma-invm" - GM4 or GM5 events, for which the largest energy cluster had a cluster mass greater than $100 \mathrm{MeV}$. Further, there were < 5 CCAL clusters, and $\mathcal{M} \geq 2.0 \mathrm{GeV}$.

25. "cmb-invm" - GM4 or GM5 events, for which the second largest energy cluster had a greater than $100 \mathrm{MeV}$ cluster mass. Also, there were < 5 CCAL clusters, and $\mathcal{M} \geq 2.0 \mathrm{GeV}$. 
26. "invmass" - GM4 or GM5 events for which $\mathcal{M} \geq 2.0 \mathrm{GeV}$, but no cluster masses > $100 \mathrm{MeV}$, and which do not satisfy either the "gold gg" or "good gg" triggers.

27. "eta etot" - GM5 events which have at least one exclusive $\eta,<6$ CCAL clusters, and $\mathcal{M}<2.0 \mathrm{GeV}$.

28. "pi etot" - GM5 events which have at least one exclusive $\pi^{0},<6$ CCAL clusters, and $\mathcal{M}<2.0 \mathrm{GeV}$.

29. "cma-etot" - GM5 events for which the largest energy cluster had a cluster mass greater than $100 \mathrm{MeV},<5 \mathrm{CCAL}$ clusters, and $\mathcal{M}<2.0 \mathrm{GeV}$.

30. "cmb-etot" - GM5 events for which the second largest energy cluster was split into two daughters, $<5$ CCAL clusters, and $\mathcal{M}<2.0 \mathrm{GeV}$.

31. "etot-soft" - GM5 events for which $\mathcal{M}<2.0 \mathrm{GeV}$, in which no cluster had a cluster mass $>100 \mathrm{MeV}$, and no exclusive $\pi^{0}$ or $\eta$ was found.

32. "neut" - Any neutral event not tagged by the other triggers."

Events which PRUDE analyzes are each assigned a 16 bit word which is written into the event record to identify the PRUDE trigger. In the offline analysis, it is then possible to divide the data into subsets simply by cutting on this 16-bit PRUDE ID. Offline selection of events with a particular PRUDE ID is a very fast method of accumulating a large set of events satisfying that trigger.

PRUDE triggers 16-23 and 27-28 are "physics" triggers. The others are primarily used for checking efficiencies of offline reconstruction. For example, a selection of 
events with the "cma" and "cmb" triggers (24-25 and 29-30) may be used to check the efficiency of the offline algorithm for allocating energy in the CCAL to clusters. (see Sec. 6.1.4) The other "non-physics" triggers are used in a similar manner. For this dissertation, all the data came from the "gold gg" PRUDE trigger (entry \#20 in Table 5.7).

\subsection{Luminosity DAQ}

The data acquisition for the luminosity monitor is separate from that for the rest of the experiment, and we describe it briefly here. Signals from each of the three detectors are input into an ORTEC AD413A ADC. An ORTEC HM413 histogramming memory module is employed as a readout controller for the ADC. It is effectively equivalent to four individual multichannel analyzers. Each channel has 24-bit capacity $(16,777,215$ counts). Each ADC output operation passes the singles spectrum from one of the LM detectors to the HM413, which accumulates a 512-bin spectrum for each.

The HM413 communicates with the run control computer via CAMAC and the Jorway branch as described in Sec. 5.1. Every two minutes, and also at the start and finish of a run, the HM413 is polled for its contents and then cleared. The three 512-bin spectra are written to disk. At the same time, the experimental dead-time is written to the same file. The dead-time percentage is defined as the ratio between the number of Gatemaster triggers written to tape or disk and the total Gatemaster strobes received. The luminosity can be normalized to reflect the experiment's 


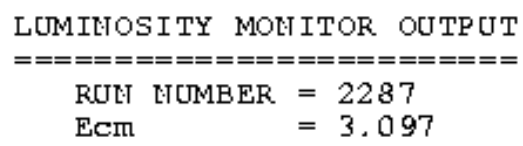

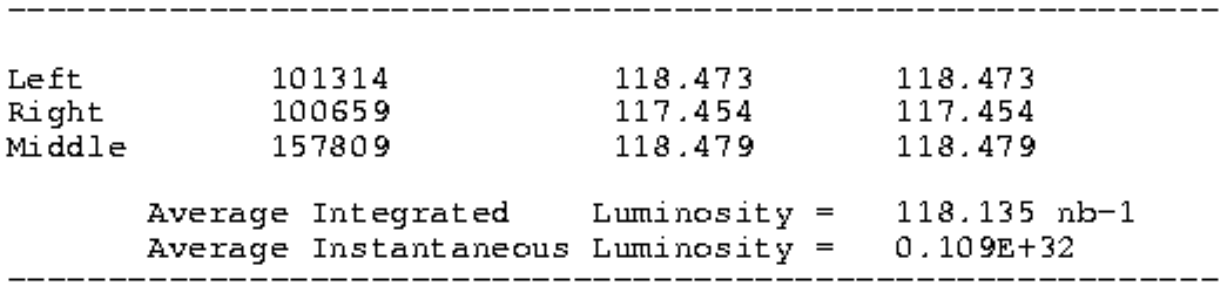

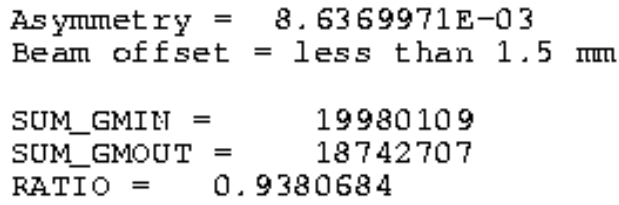

Figure 5.8: Output from the luminosity program from one of the E835 $\mathrm{J} / \psi$ runs.

"effective" luminosity using this ratio.

At the end of a run a simple program was used to determine the integrated and average instantaneous luminosity for the run, by summing all the relevant histograms, fitting the observed spectrum, and subtracting the fit background to determine the number of recoil protons for the run, and consequently the luminosity. The output from this program for a typical E835 run is shown in Fig. 5.8. 


\section{Chapter 6}

\section{Identification of Photons}

The charmonium states for which we present results in this dissertation $\left(\eta_{c}, \eta_{c}^{\prime}, \chi_{0}\right.$

and $\chi_{2}$ ) are detected by their decay into two photons. We discuss in this chapter the identification of photons using the E835 detector and data acquisition systems. We describe the means by which we determine the energies and positions of particles which deposit energy in the CCAL, and then address the criteria by which we identify a particular energy deposit as being due to a photon.

\subsection{CCAL Clustering}

Electromagnetic showers initiated by individual electrons or photons striking the CCAL are contained in a small region of the calorimeter, which includes a central block and its eight nearest neighbors. These regions are called clusters. When clusters are isolated from each other (i.e. with centers separated by at least two 
rings and two wedges) the reconstruction of their position and energy is simple. However, complications arise in instances in which two photons or electrons strike the CCAL in adjacent blocks. In such cases the energy in blocks common to the two clusters must be shared in order that proper energy and position determinations can be made for each incident particle. Further, as $\sqrt{s}$ is increased, it becomes increasingly probable that symmetric decays of $\pi^{0}$ s produced in the $\bar{p} p$ interactions result in two photons so close to one another that they are not simply resolvable (i.e. they appear to form a single cluster). It is therefore necessary to have an algorithm which can identify such problem clusters, and which is able to separate, or "split" such clusters appropriately.

In each of the above cases, assignment of position and energy to a cluster is not a simple matter of calculating a center of gravity and a sum over all the blocks in the cluster. The steel partitions among the CCAL blocks form a dead layer which absorbs energy, which must also be accounted for. We will describe in this section the primary algorithm by which clusters in the CCAL are identified and assigned energy and position, and the secondary algorithms which were developed to deal with isolated clusters, clusters which share common blocks, and clusters which must be split into two.

\subsubsection{Main Clustering Algorithm}

The main clustering algorithm begins identifying all CCAL blocks which contain more energy than their eight nearest neighbors. These blocks, if they contain more than $5 \mathrm{MeV}$, are identified as "cluster seeds". This seed threshold of $5 \mathrm{MeV}$ is 
associated with a cluster threshold of $20 \mathrm{MeV}$. That is, a seed will not be treated as the center of a cluster unless its eight nearest neighbors contain an additional 15 $\mathrm{MeV}$. These thresholds reduce the likelihood that random noise in the calorimeter is incorrectly classified as a cluster.

As the next step, the distance is calculated in block units between each cluster seed in relation to every other cluster seed. The distance D is defined as $\sqrt{\Delta R^{2}+\Delta W^{2}}$, where $\Delta R$ is the distance between cluster seeds in ring units, and $\Delta W$ the distance in wedge units. This is illustrated in Fig. 6.1. Clusters whose nearest neighbor is farther away than $D_{\min }=\sqrt{18}$ are designated as "isolated". Their analysis then proceeds via the isolated cluster routine, which is described in Sec. 6.1.2. If a cluster has one or more neighbors which are as close or closer than $D_{m i n}$, it is marked as "non-isolated". The analysis of these clusters proceeds with the cluster energy sharing procedure which is described in Sec. 6.1.3.

Once each cluster has been identified as isolated or non-isolated, a check is performed on all isolated clusters to determine whether they are due to two incident photons from a symmetric $\pi^{0}$ decay, rather than to a single photon. In such cases, as described earlier, the two photons strike within the same $3 \times 3$ grid of blocks, and the distribution of energy is such that there is only one discernible local maximum. However, most of these "merged" photon clusters are resolvable because energy is spread over a region larger than the $3 \times 3$ square of blocks which surround the single maximum. In order to separate clusters due to single $\gamma$ or $e$ from these due to "merged" photons, an approximation to the invariant mass may be calculated for a cluster. This "cluster mass" is calculated over the 5x5 square of blocks for all 


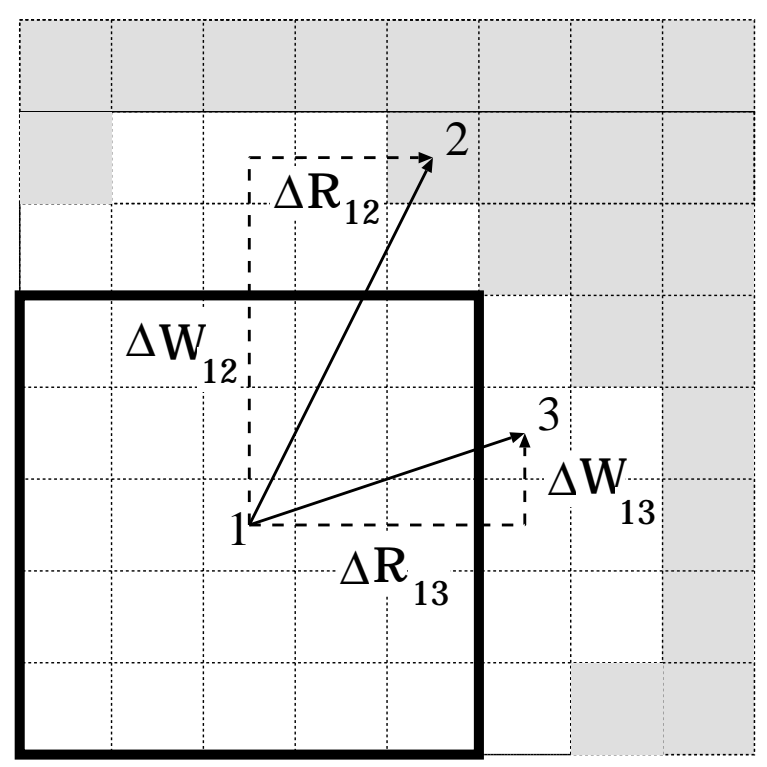

Figure 6.1: Depiction of isolated and non-isolated cluster seeds. Shown is the 5x5 grid around seed \#1, and two nearby seeds \#2 and \#3. Using the definitions in the text, seed \#2 has $D_{12}=\sqrt{2^{2}+4^{2}}=\sqrt{18}$. It, as well as other seeds in shaded blocks, is considered 'isolated' from \#1. Seed \#3 has $D_{13}=\sqrt{3^{2}+1^{2}}=\sqrt{10}$, and along with possible seeds in other unshaded blocks, it is not considered 'isolated'.

isolated cluster seeds in the CCAL.

For each block in the $5 \times 5$ region around a cluster seed, there are two angles, $\phi(r, w)$ and $\theta(r, w)$, which are measured relative to the $\bar{p} p$ interaction point. We can then calculate an invariant mass for the cluster, using the energy in each block as the fourth component of a four vector directed from the origin to the block center, thus:

$$
\begin{aligned}
E_{25} & =\Sigma_{5 x 5}[E(r, w)] \\
P_{x} & =\Sigma_{5 x 5}[E(r, w) \times \sin \theta(r, w) \cos \phi(r, w)]
\end{aligned}
$$




$$
\begin{aligned}
P_{y} & =\Sigma_{5 x 5}[E(r, w) \times \sin \theta(r, w) \sin \phi(r, w)] \\
P_{z} & =\Sigma_{5 x 5}[E(r, w) \times \cos \theta(r, w)] \\
M_{\text {cluster }} & =\sqrt{E_{25}^{2}-P_{x}^{2}-P_{y}^{2}-P_{z}^{2}} .
\end{aligned}
$$

If this "cluster mass" is greater than $100 \mathrm{MeV}$, it is possible that the cluster is due to merged photons from a $\pi^{0} \rightarrow \gamma \gamma$ decay. For such clusters, a new second cluster seed is created, in the block with the next highest energy in the cluster. It has been found empirically, by studying clusters due to $J / \psi \rightarrow e^{+} e^{-}$events, that a cluster due to a single incident electron should have cluster mass less than about $100 \mathrm{MeV}$. In principle, single photons should satisfy the same criterion.

The distribution of cluster masses for neutral clusters in typical run at $\eta_{c}$ is shown in Fig. 6.2, where it is compared to the cluster mass distribution for electrons from $J / \psi \rightarrow e^{+} e^{-}$. Clusters whose mass is found to be greater than $100 \mathrm{MeV}$ are analyzed in the "cluster splitting algorithm" which is described in Sec. 6.1.4.

\subsubsection{Isolated Cluster Analysis}

The basic idea behind the isolated cluster energy and position evaluation is common to all experiments which use segmented calorimeters. To first order, the energy $E_{0}$ of a cluster is determined by summing the energies in each of the $3 \times 3$ set of blocks, and the positions $\left(R_{0}, W_{0}\right)$ in block units are calculated using simple weighted averages:

$$
E_{0}=\sum_{i=1}^{9} E_{i}, \quad R_{0}=\frac{\sum_{i=1}^{9} E_{i} \Delta R_{i}}{E_{0}}, \quad W_{0}=\frac{\sum_{i=1}^{9} E_{i} \Delta W_{i}}{E_{0}} .
$$

Here, the $\Delta R_{i}$ and $\Delta W_{i}$ are the positions of each block in the $3 \times 3$ grid relative to the central block, i.e. $\Delta R_{i}, \Delta W_{i} \in(-1,0,1)$. Thus, $R_{0}$ and $W_{0}$ range from -.5 to 


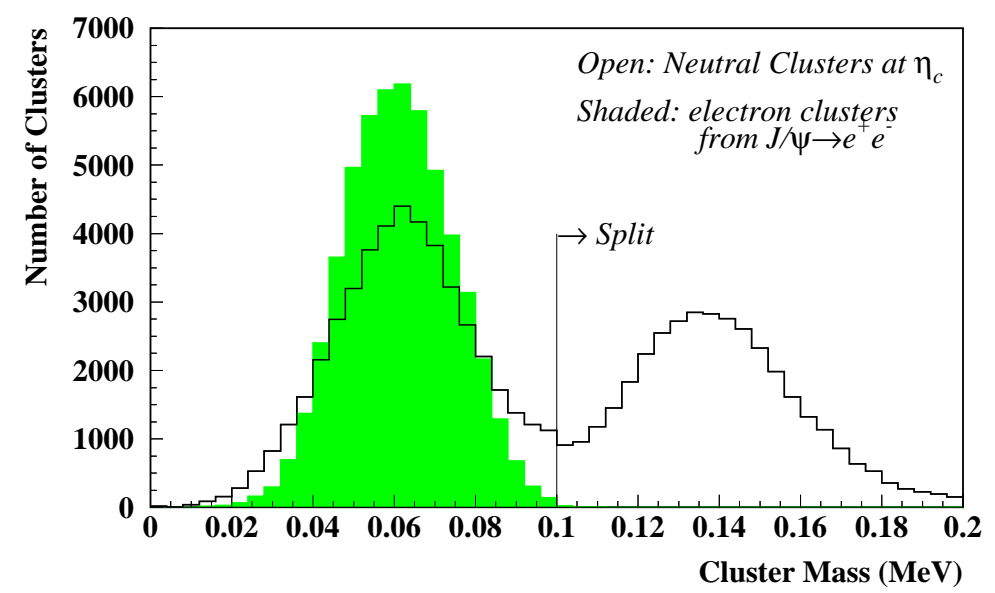

Figure 6.2: Comparison of cluster masses for electrons (shaded) from $J / \psi \rightarrow e^{+} e^{-}$ and for neutral clusters at $\eta_{c}$ (open). The neutral cluster sample shows evidence for "merged" $\gamma$ 's due to symmetric $\pi^{0}$ decay.

+.5 , with $\left(R_{0}, W_{0}\right)=(0,0)$ corresponding to the center for the central block.

Electromagnetic showers in typical calorimeter materials such as lead glass are characterized by a narrow shower deposit and long exponential tails. Thus the first order centroids $R_{0}$ and $W_{0}$ are dominated by the large energy deposit in the central block. The true position of the shower, then, must be obtained through corrections to the centroid which depend on a formula for the transverse shower development in the lead glass, whose parameters must be determined empirically for any particular calorimeter. In terms of the first order cluster centroids $R_{0}$ and $W_{0}$, the formula for the true position $R$ and $W$ can be written in terms of four parameters, $\alpha_{R}, \lambda_{A R}, \beta_{R}$ and $\lambda_{B R}$ :

$$
R=\left(\alpha_{R} \times\left(1-e^{\left|R_{0}\right| / \lambda_{A R}}\right)+\beta_{R} \times\left(1-e^{\left|R_{0}\right| / \lambda_{B R}}\right)\right)
$$




$$
W=\left(\alpha_{W} \times\left(1-e^{\left|W_{0}\right| / \lambda_{A W}}\right)+\beta_{W} \times\left(1-e^{\left|W_{0}\right| / \lambda_{B W}}\right)\right)
$$

In preparation for E760, three CCAL wedges were taken to the Brookhaven National Laboratory to be tested using a $5 \mathrm{GeV}$ electron beam. [46] The actual position of electrons incident upon the blocks in this test setup was recorded by wire chambers. Parameters for Eqs. 6.8 were determined empirically by comparison of the actual beam particle position to the first order calculation of cluster centroids.

As stated earlier, the presence of steel in the interstitial spaces must be corrected for in order to obtain the correct energy for a cluster. This correction has been parameterized in terms of the positions $R^{\prime}$ and $W^{\prime}$, which are measured from the edges of the block, i.e. $R^{\prime}=R-R_{\text {center }}$ and $W^{\prime}=W-W_{\text {center }}$ :

$$
E=E_{0} /\left[\left(1-\zeta_{R} * e^{-\left|R^{\prime}\right| / \xi_{R}}\right)\left(1-\zeta_{A W} * e^{-\left|W^{\prime}\right| / \xi_{A W}}-\zeta_{B W} * e^{-\left|W^{\prime}\right| / \xi_{B W}}\right)\right] .
$$

The parameterization in the ring direction is different from that in the wedge direction because of the different amount of steel separating rings and wedges (see Sec. 4.4.3). Furthermore, correction due to inter-ring material, because of the staggering of blocks (see Fig. 4.18), is parameterized differently depending upon whether the position $R$ lies in the upstream or downstream half of the ring. $\left(\zeta_{R}=\zeta_{d R}\right.$ or $\zeta_{u R} ; \xi_{R}=\xi_{d R}$ or $\left.\xi_{u R}\right)$

All the parameters discussed in this section were determined during the electron beam tests at BNL. Their values appear in Table 6.1. 
Table 6.1: Parameters for correction of initial ring and wedge position and energy estimates.

\begin{tabular}{|cc|cc||cc|cc|}
\hline Parameter & Value & Parameter & Value & Parameter & Value & Parameter & Value \\
\hline$\alpha_{R}$ & 0.260 & $\alpha_{W}$ & 0.314 & $\zeta_{d R}$ & 0.061 & $\zeta_{A W}$ & 0.147 \\
$\lambda_{A R}$ & 0.032 & $\lambda_{A W}$ & 0.040 & $\xi_{d R}$ & 0.136 & $\xi_{A W}$ & 0.020 \\
$\beta_{R}$ & 0.257 & $\beta_{W}$ & 0.197 & $\zeta_{u R}$ & 0.086 & $\zeta_{B W}$ & 0.159 \\
$\lambda_{B R}$ & 0.186 & $\lambda_{B W}$ & 0.172 & $\xi_{u R}$ & 0.051 & $\xi_{B W}$ & 0.078 \\
\hline
\end{tabular}

\subsubsection{Non-Isolated Cluster Analysis}

In the case of two clusters $(j=1,2)$ which are close enough so that sharing energy between clusters needs to be considered, initial cluster energies and positions are calculated using weighted energies in each block of the cluster. Thus, Eqs. 6.6 are replaced by:

$$
E_{0 j}=\sum_{i=1}^{9} \eta_{i j} E_{i}, \quad R_{0 j}=\frac{\sum_{i=1}^{9} \eta_{i j} E_{i} \Delta R_{i j}}{E_{0 j}}, \quad W_{0 j}=\frac{\sum_{i=1}^{9} \eta_{i j} E_{i} \Delta W_{i j}}{E_{0 j}} .
$$

i.e. the formulae used are the same as if an energy $\eta_{i j} E_{i}$ was deposited in the $i$ th block, rather than the measured energy $E_{i}$. The weights $\eta_{i j}$ are dependent upon the position of the $i$ th block from the centroid of the $j$ th cluster, ( $j=$ only 1 or 2$)$ and are normalized so that $\eta_{i 1}+\eta_{i 2}=1$. They are calculated according to the following iterative procedure.

Initially, the weights $\eta_{i j}$ are set to 1 for each block shared between the two clusters. (so the normalization rule does not hold in the first iteration) Shared blocks are those which lie within the $5 \times 5$ region of both clusters. For other blocks, the $\eta_{i j}$ is set to 1 for the cluster which is closer. $E_{0 j}, R_{0 j}$ and $W_{0 j}$ are then calculated according to these weights, as a first approximation. 
After defining the distance $D_{i j} \equiv \sqrt{\left(\Delta R_{i j}\right)^{2}+\left(\Delta W_{i j}\right)^{2}}$ between the $i$ th block and the centroid of the $j$ th cluster in block units, the $\eta_{i j}$ are defined:

1. If both $D_{i 1}$ and $D_{i 2}$ are $\leq \sqrt{3.5}$ blocks, the weights $\eta_{i j}$ take the following form:

$$
\begin{aligned}
\eta_{i 1} & =\frac{E_{1} e^{-\left(\Delta_{1} / 0.17\right.}}{E_{1} e^{-\Delta_{1} / 0.17}+E_{2} e^{-\Delta_{2} / 0.17}} \\
\eta_{i 2} & =\frac{E_{2} e^{-\Delta_{2} / 0.17}}{E_{1} e^{-\Delta_{1} / 0.17}+E_{2} e^{-\Delta_{2} / 0.17}}
\end{aligned}
$$

with $\Delta_{1} \equiv\left|\Delta R_{i 1}\right|+\left|\Delta W_{i 1}\right|$ and $\Delta_{2} \equiv\left|\Delta R_{i 2}\right|+\left|\Delta W_{i 2}\right|$. The 0.17 in the exponents is the characteristic transverse shower decay length in block units, which was empirically determined.

2. If, however, $D_{i 1} \leq D_{i 2}$ and $D_{i 1} \leq \sqrt{4.5}$ blocks, then

$$
\eta_{i 1}=1 \quad \text { and } \quad \eta_{i 2}=0
$$

and vice versa.

3. Finally, if $D_{i 1} \geq \sqrt{4.5}$ blocks and $D_{i 2} \geq \sqrt{4.5}$ blocks, then

$$
\eta_{i 1}=0 \quad \text { and } \quad \eta_{i 2}=0
$$

Given these weights $\eta_{i j}$, new cluster centroid positions and energy estimates are calculated according to Eq. 6.10. If the change in either cluster's energy (from the previous iteration) is greater than $30 \mathrm{MeV}$, or if the change in either cluster's position is greater than .005 blocks in either dimension, another iteration is performed. This procedure usually converges after 2 or 3 iterations. 


\subsubsection{Cluster Splitting Analysis}

In order to split clusters whose cluster mass is over $100 \mathrm{MeV}$, a choice must be made as to the locations of the "daughter" cluster seeds. In our algorithm the initial position of one seed is the center of the block which represents the "parent" cluster maximum. The second seed is placed at the center of the next highest energy

block in the "parent" cluster - which must be one of the eight nearest neighbors of the first seed.

Once the seeds have been chosen, energy and position corrections for the two "daughter" clusters uses an iterative algorithm nearly identical to that for the energy sharing procedure (Sec. 6.1.3). The only differences between the two routines is that:

- The central maximum is not used in determining initial positions and energies.

- The limit of $\sqrt{4.5}$ blocks presented in items 2 and 3 of the energy sharing procedure (Eq. 6.13 and 6.14 ) is changed to $\sqrt{8.5}$ blocks.

\subsection{Photon Identification}

The identification of a CCAL cluster as a photon is based on two simple criteria:

1. The cluster must not be associated with a charged track; that is, the cluster must have no corresponding "track" in the scintillator hodoscopes. Specifically, there can be no coincidence in $H 1 \cdot H 2^{\prime}$. Recall that an $H 1 \cdot H 2^{\prime}$ coincidence removes an event at the trigger level through the Neutral Veto.

2. It must have a cluster mass of less than $100 \mathrm{MeV}$. 


\subsubsection{Possible Misidentification of $\gamma^{\text {'s }}$}

Given the above conditions for calling a cluster a photon, there are two possible phenomena which in principle can give rise to a false identification.

1. An electron which did not fire the Neutral Veto This phenomenon should be rare, but must be considered. The CCAL responds the same way to a single photon as it does a single electron. Shower shapes in lead glass are known to be very similar whether the incident particle is a photon or an electron. Without information from the charged tracking, there is no means of distinguishing between an electron cluster and a photon cluster. In an $e^{+} e^{-}$event, though, the identification of the second electron can help remove this ambiguity.

2. A $\pi^{0}$ which decays symmetrically, such that the showers of its two photons form a single cluster in the CCAL. This background is taken care of in large part by an evaluation of the cluster mass, which was introduced in the previous section. If $M_{c l} \geq 100 \mathrm{MeV}$, (see Fig. 6.2) the cluster is split using the algorithm described in Sec. 6.1.4. If an event contains a cluster which has been split in this manner, it is removed from the $\gamma \gamma$ data sample.

It should be noted that there is a small chance that some of the $\gamma$ candidate clusters arise from a $\pi^{0}$ whose photons have merged, but which gave a cluster mass $M_{c l} \leq 100 \mathrm{MeV}$ (and was therefore not split). This might suggest lowering the cut below $100 \mathrm{MeV}$. It was found, however, that changing the value of the splitting threshold from $M_{c l} \leq 100 \mathrm{MeV}$ does not improve the signal-to-background ratio at $\eta_{c}$. 
Table 6.2: Estimated thicknesses (in radiation lengths) of the E835 inner detectors.

\begin{tabular}{|lc||l|l|l||l|}
\hline Detector & & Material & $\begin{array}{l}\text { thickness }(\mathrm{mm}) \\
\text { at normal } \\
\text { incidence }\end{array}$ & $\begin{array}{l}\text { Radiation } \\
\text { Lengths }\end{array}$ & Total \\
\hline Hodoscope & $H 1$ & scintillator & 2 & $0.47 \%$ & $0.47 \%$ \\
Straws & $S C 1$ & Mylar & 0.3 & $0.1 \%$ & $0.57 \%$ \\
Hodoscope & $H 2^{\prime}$ & scintillator & 4 & $0.94 \%$ & $1.51 \%$ \\
Silicon & $S I$ & various & - & $2.0-4.5 \%$ & $3.5-6.0 \%$ \\
Straws & $S C 2$ & Mylar & 0.3 & $0.1 \%$ & $3.6-6.1 \%$ \\
Fiber & $S F$ & various & - & $\sim 1 \%$ & $\sim 4.6-6.1 \%$ \\
Hodoscope & $H 2$ & scintillator & 4 & $0.94 \%$ & $\sim 5.6-7.1 \%$ \\
Cerenkov & ${ }^{2} C$ & various & - & $\sim 2.5 \%$ & $\sim 8.1-9.6 \%$ \\
\hline
\end{tabular}

\subsubsection{Inefficiency due to $\gamma$ conversions}

Real $\gamma \gamma$ events may not be identified as such if one or the other photon converts in material between the interaction region and the CCAL. An appropriate set of cuts can be made, however, so that these events are retained. This requires a study of such conversion events. We can then estimate the inefficiency, if any, due to photon conversions. For reference, we present in Table 6.2 the estimated thickness (for normal incidence) for all inner detectors.

\section{Beampipe conversions and in $H 1$}

If photon in a $\gamma \gamma$ event converts in the beampipe, (rad length $\lesssim 1 \%$ ) the event will always be lost. Since the beam pipe lies inside the radius of $H 1$, a beampipe conversion will result in a charged track in the hodoscopes, and thus the neutral veto will be fired. It is also possible that a $\gamma$ conversion which occurs in $H 1$ itself can fire the neutral veto. If the conversion occurs early enough in the scintillator 
thickness, it is possible that the resulting "double track" will give a sufficiently large pulse height in $H 1$ that, with the corresponding signal in $H 2^{\prime}$, will fire the neutral veto. These events will be lost as well, and being indistinguishable from beam pipe conversions.

The probability of a these two types of conversions was measured in E760 to be $\sim 1.1 \%$ per photon, and this value has been confirmed in E835 data. [47]

\section{Conversions after $H 1$}

Conversions which occur after $H 1$ will not fire the neutral veto, and thus make up part of the neutral trigger data set. These conversions may occur in any of the inner detectors which lie outside of $H 1$ : SC1, H2', SIL, SC2, SF or H2 (See Fig. 4.12). The Straws (SC1 and $S C 2)$ and the Scintillating Fiber Tracker $(S F)$ do not comprise much material at all, $(\sim 1.2 \%$ of a rad length; see Table 6.2$)$ and therefore are not expected to contribute much in the way of conversions, and are not considered here. However, the Hodoscopes (each $\sim 1 \%$ ) and Silicon barrel $(\sim 2.0-4.5 \%)$ can be expected to cause conversions, and this possibility has been examined.

Photons which convert after H1 can be identified by their signal in the hodoscopes, or in the Čerenkov detector. The pulse height spectra (in equivalent MIP units) for hodoscopes $H 1, H 2^{\prime}$ and $H 2$, and for the Čerenkov detector, are shown in Fig. 6.3-6.6, for events recorded in the $J / \psi$ and ${ }^{1} P_{1}$ regions. In these figures, the shaded histogram shows the pulse height for events in the neutral set - i.e. "gold gg" events taken at the ${ }^{1} P_{1}$. The unshaded histogram shows the same for events 


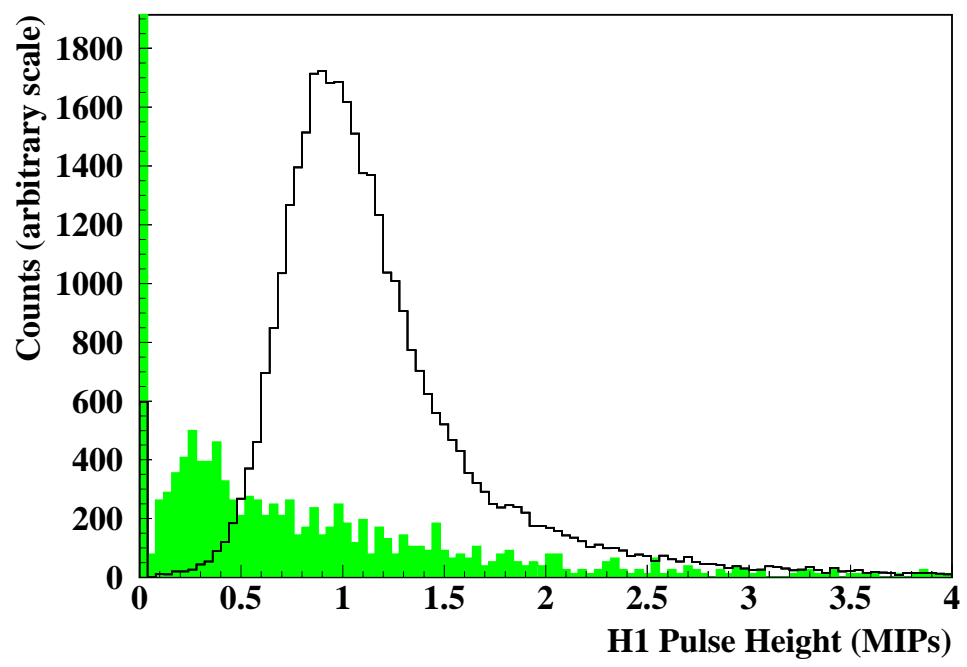

Figure 6.3: Pulse height spectra (in MIP units) from $H 1$ for electrons (open histogram) and photons (shaded histogram).

from the charged trigger $-e^{+} e^{-}$events taken at $J / \psi$. The shaded and open pulse height spectra have been arbitrarily normalized, in order to make comparison of the shapes of the two distributions easier.

In the $H 1$ spectrum (Fig. 6.3) there is no identifiable enhancement for neutral events in the 1.5 - 3.0 MIP region. This indicates that the fraction of events representing $\gamma^{\prime}$ s which converted prior to incidence on $H 1$ is essentially nil as expected. A clear, though small, enhancement in the $H 2^{\prime}$ pulse height spectrum in the 1.5-3.0 MIP region is, however, seen after we have greatly magnified the count scale. (See Fig. 6.4) This indicates that the fraction of possible conversions which occur inside the radius of $H 2^{\prime}$ is quite small. In contrast, "double" tracks (pulse heights in the range 1.5-3.0 units) are prominent in the $H 2$ and Čerenkov plots. (Figs. 6.5 and 


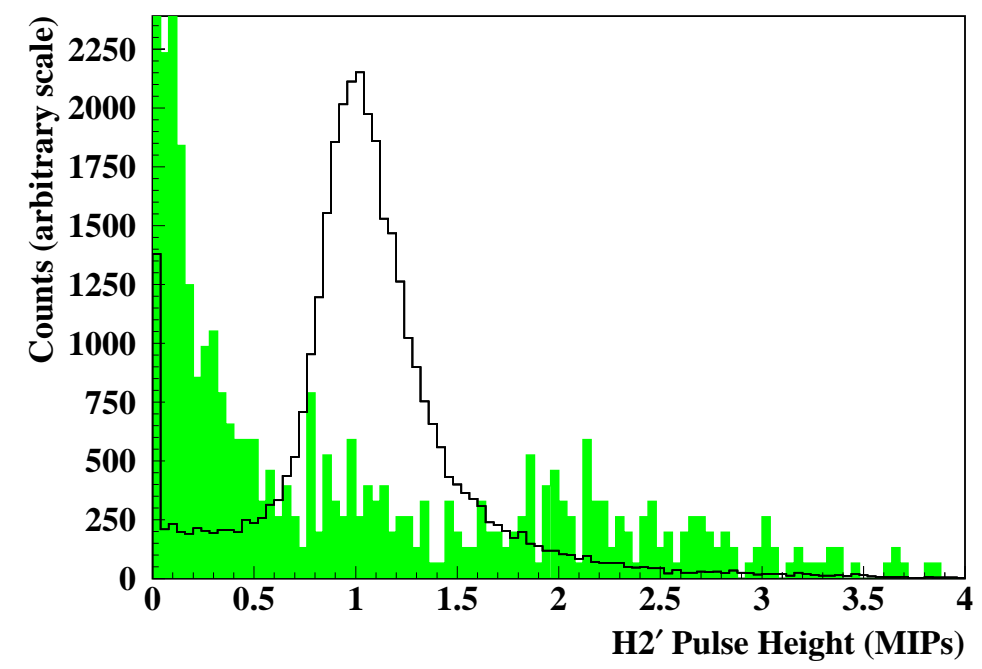

Figure 6.4: Pulse height spectra (in MIP units) from $H 2^{\prime}$ for electrons (open histogram) and photons (shaded histogram).

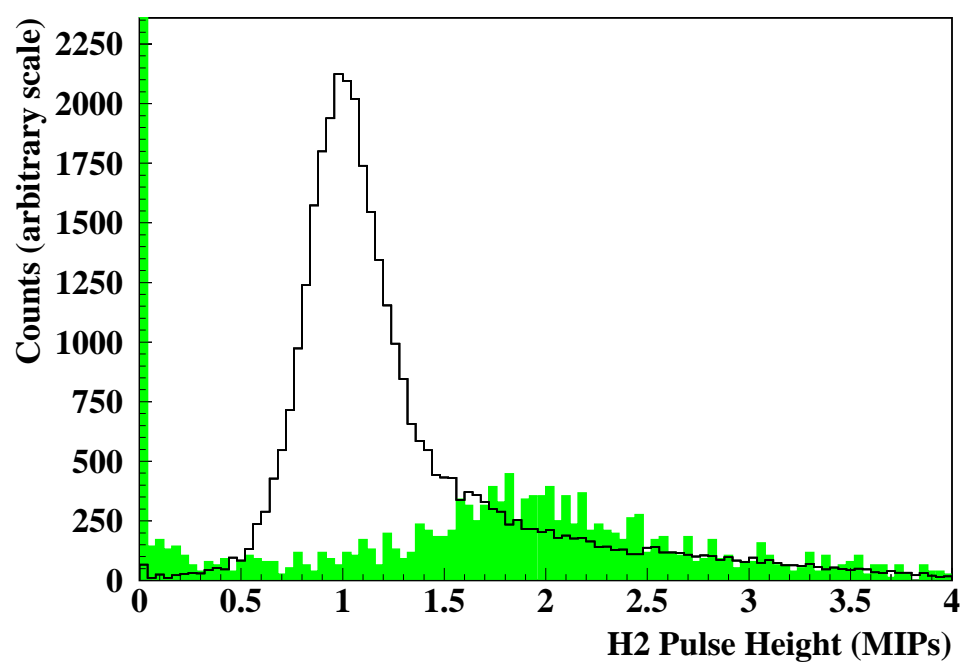

Figure 6.5: Pulse height spectra (in MIP units) from $H 2$ for electrons (open histogram) and photons (shaded histogram). 
6.6) This observation is consistent with the hypothesis (see Sec. 4.4.1) that most of the conversions we detect occur in the silicon barrel, which lies just inside $H 2$.

\section{Do we lose these events?}

The primary concern we have regarding conversions is whether or not we unwittingly remove them from our event sample, due to the cuts we make. As outlined in the next chapter, the only cut which we use to remove charged final state events is a cut on the pulse heights in $H 1$ and $H 2^{\prime}$. We have shown that most conversions occur beyond $H 2^{\prime}$. Thus most events containing $\gamma$ conversions should pass cuts on $H 1$ and $H 2^{\prime}$ pulse heights. In order to show that this is indeed the case, we have studied the effect of applying the $H 1$ and $H 2^{\prime}$ cuts to events containing conversions.

For the purpose of this study, we have defined a converted photon as a CCAL cluster having pulse heights in corresponding elements of both $H 2$ and $\breve{C}$ between 1.5 and 3 MIP units. We have examined events in the $\eta_{c},{ }^{1} P_{1}$ and $\eta_{c}^{\prime}$ regions, comparing the total number of 2-cluster events in the "gold gg" trigger sample to the number of conversion candidates as defined above. We also list the number of these candidates which are removed due to the cuts on the pulse heights in $H 1$ and $H 2^{\prime}$. The number of conversions, $\sim 8 \%$ on average, is somewhat large, but it is of no consequence, since the number events which we remove is a very small proportion of the total number of $\gamma \gamma$ candidate events $(\leq 0.6 \%)$. 


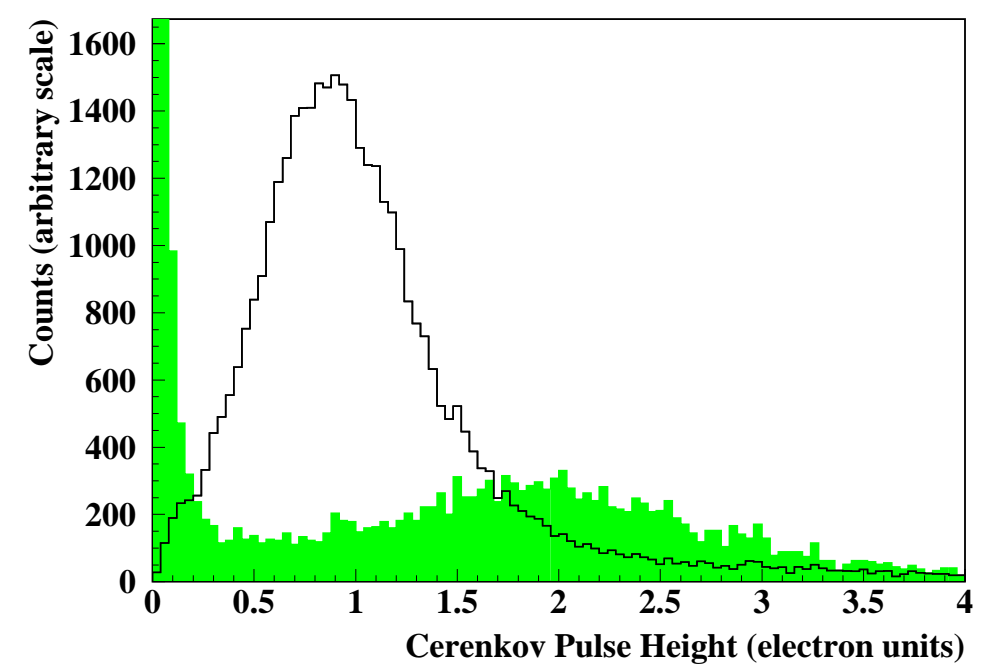

Figure 6.6: Pulse height spectra (in electron units) from the Čerenkov counter for electrons (open histogram) and photons (shaded histogram).

Table 6.3: Fraction of $\gamma$ candidates which appear as "conversions", having pulse heights of 1.5 to 3.0 MIPS in both $H 2$ and Č.

\begin{tabular}{|l|c|cc|cc|}
\hline $\begin{array}{l}\text { Resonance } \\
\text { region }\end{array}$ & $\begin{array}{c}\gamma \\
\text { candidates }\end{array}$ & $\begin{array}{c}\text { "Conv." } \\
(\text { all })\end{array}$ & $\begin{array}{c}\text { "Conv." } \\
\text { as } \% \\
\text { of total }\end{array}$ & $\begin{array}{c}\text { "Conv." } \\
\text { retained after } \\
H 1 \cdot H 2^{\prime} \text { cut }\end{array}$ & $\begin{array}{c}\text { Conv. lost } \\
\text { as } \% \\
\text { of total }\end{array}$ \\
\hline \hline$\eta_{c}$ & 5086 & 380 & $7.5 \pm 1.3$ & 370 & $0.20 \pm 0.06$ \\
${ }^{1} P_{1}$ & 26238 & 2004 & $7.6 \pm 0.6$ & 1870 & $0.51 \pm 0.04$ \\
$\eta_{c}^{\prime}$ & 26940 & 2250 & $8.4 \pm 0.5$ & 2093 & $0.58 \pm 0.05$ \\
\hline
\end{tabular}




\section{Chapter 7}

\section{Selection and Analysis of $\gamma \gamma$ Events}

\subsection{Initial Data Selection}

The $\gamma \gamma$ events analyzed for this dissertation are collected in the "gold gg" data stream. The requirements for this trigger subset are loose - so that $\gamma \gamma$ events are accepted with $100 \%$ efficiency. As a result, the vast majority of the initial "gold gg" data sample are $\pi^{0} \pi^{0}, \pi \eta$ and $\eta \eta$ events, which constitute the main sources of background to the $\gamma \gamma$ final state. This is illustrated in Fig. 7.1, in which we have plotted for each "gold gg" event the invariant mass combinations between each of the two largest energy clusters $\left(\gamma_{1}\right.$ and $\left.\gamma_{2}\right)$ and additional clusters in the calorimeter. $\pi^{0} \pi^{0}, \pi^{0} \eta$ and $\eta \eta$ events are all readily apparent. We describe below the cuts which we use to extract a final selection of $\gamma \gamma$ candidate events from this background. 

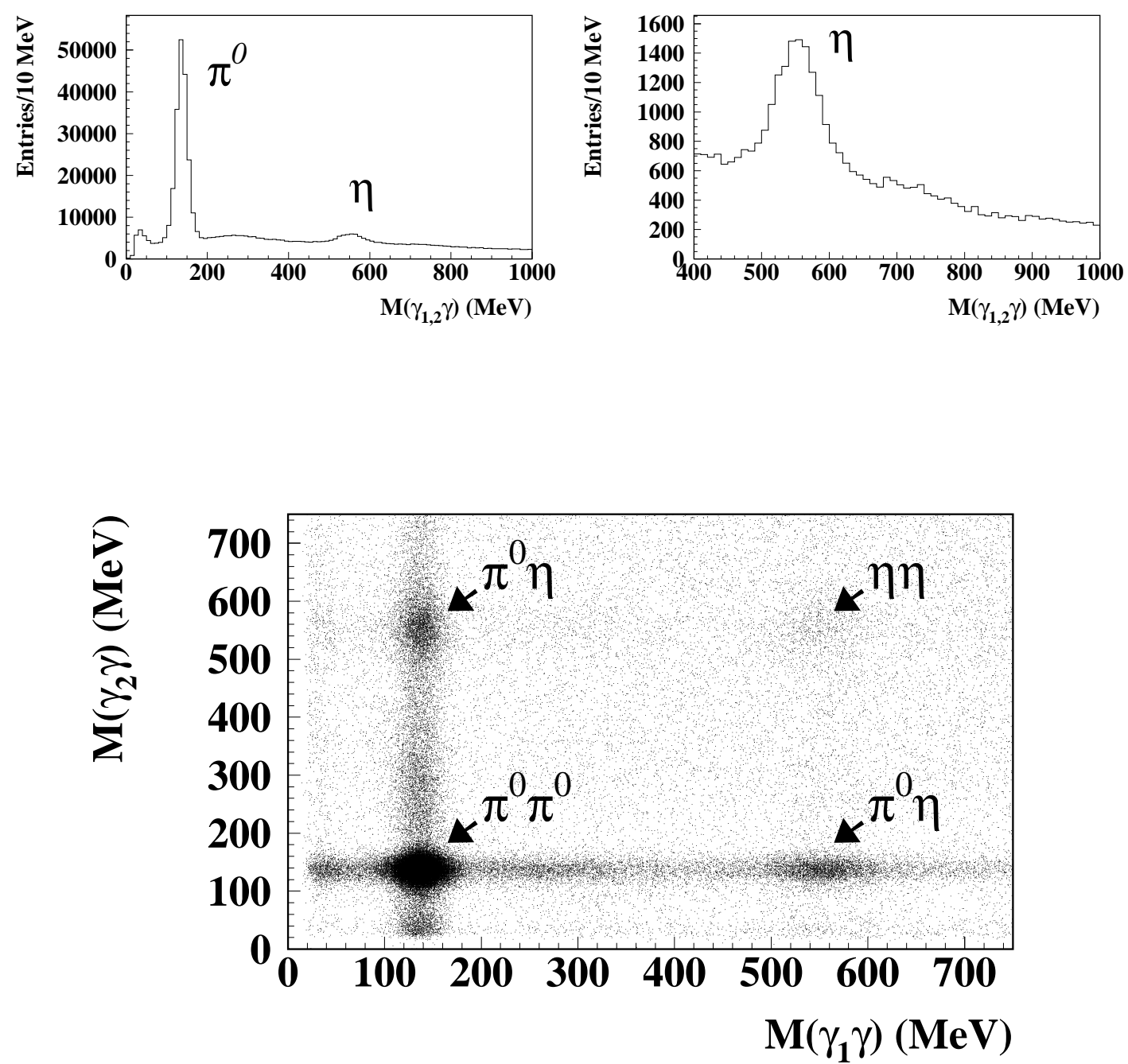

Figure 7.1: Invariant mass combinations calculated with each $\gamma$ candidate and untimed extra clusters in the CCAL, showing $\pi^{0}$ (upper left), $\eta$ (upper right), and (bottom) the dominance of $\pi^{0} \pi^{0}$ events in the "gold gg" trigger sample. 


\section{2 $\gamma \gamma$ Event Selection Criteria}

Cuts are made using the following information:

- Cluster time

- Invariant mass between $\gamma$ candidate cluster and "extra" CCAL clusters

- Event kinematics $\left(\chi^{2}\right.$ probability for $4 \mathrm{C}$ kinematic fit or $\Delta \phi$ and $\Delta \theta$ )

- Polar angle $\theta$ of $\gamma$ candidates

- Charged Hodoscope $\left(H 1, H 2^{\prime}\right)$ pulse height

\subsubsection{Cluster Time Cut}

In E835 the instantaneous luminosity was significantly increased compared to E760, and in order to be able to retain real events which are contaminated with additional interactions in the same CCAL FERA gate, each CCAL block was outfitted with TDCs (see Sec. 4.4.3). The effect of this timing capability is two-fold. First, it

allows us to remove a large part of the $\pi^{0} \pi^{0}$, etc. events from the trigger sample very efficiently by cutting on the number of in-time clusters. Second, it allows us to retain the $\gamma \gamma$ events which contain "extra" CCAL clusters associated with "out-of-time" interactions.

\section{Definition of Cluster Time}

The "time" of a cluster is defined as the TDC value of the largest energy block in the cluster which has a TDC hit. Most of the time, this is the central block (i.e. the 


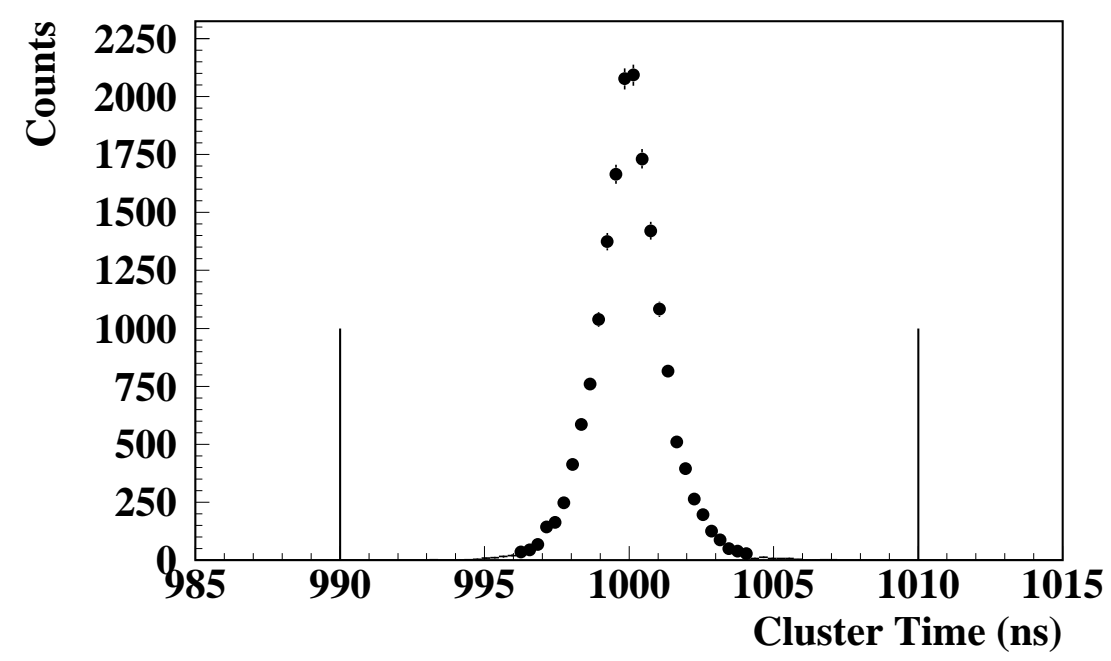

Figure 7.2: Cluster times for the two large-energy clusters in a sample of clean $\pi^{0} \pi^{0}$ events, taken at $\sqrt{s}=3576 \mathrm{MeV}$. The FWHM of this distribution is $\sim 5.8 \mathrm{~ns}$. The "in-time" window is indicated by the vertical lines.

largest energy block), but occasionally that block will have no TDC hit. In these cases, the cluster time comes from the second-largest energy block in the cluster. If no block in the cluster has a TDC hit, then its time is recorded as 0 , and the cluster is referred to as an "untimed" cluster. We define a cluster as "in-time" if its TDC signal lies within $\pm 10 \mathrm{~ns}$ of the mean event time of $1000 \mathrm{~ns}$. If a cluster's time is non-zero, but outside this 20 ns window, it is called "out-of-time".

The time for each of the two large-energy clusters in a set of clean $\pi^{0} \pi^{0}$ events (selected by requiring 4 CCAL clusters exactly, and two reconstructed $\pi^{0}$ masses 100 to $170 \mathrm{MeV}$ ) is shown in Fig. 7.2. The vertical lines in the figure define the 20ns "in-time" window. 


\section{Effect of Timing}

As mentioned earlier, the capability of timing CCAL clusters is helpful in two ways: we are able to reject events with more than two in-time clusters, and we are able to retain true $\gamma \gamma$ events which contain additional CCAL clusters which arise from noise or out-of-time interactions. Figs. 7.3 and 7.4 illustrate the effect of having this timing capability.

In Fig. 7.3 we show the number of CCAL clusters for events which pass all cuts discussed in this chapter, except the timing cut. As expected, most events have just two clusters (334/661). Fig. 7.4 shows the same for events which pass all cuts, including the timing (334/434 have just two clusters). If we had no timing capability, we would have the choice in Fig. 7.3 of accepting events which have only 2 CCAL clusters (334), or accepting events irrespective of the number of CCAL clusters they contain (661). In the former case, we would lose many good $\gamma \gamma$ events $(434-334=$ 100) due to the presence of extra clusters in the CCAL. In the latter case, we would be accepting accepting a larger amount of background $(661-434=227)$

\section{Description of the cut}

We accept only events which contain only two in-time clusters in the CCAL. These two must be the two largest energy clusters (i.e., the photon candidates). Any number of additional clusters may be present in the CCAL, but no cluster besides the $\gamma$ candidates may have a time which is in the 20 ns window from 990 ns to 1010 ns. (See Fig. 7.2)

Since we only allow two on-time clusters, the timing cut removes a large per- 


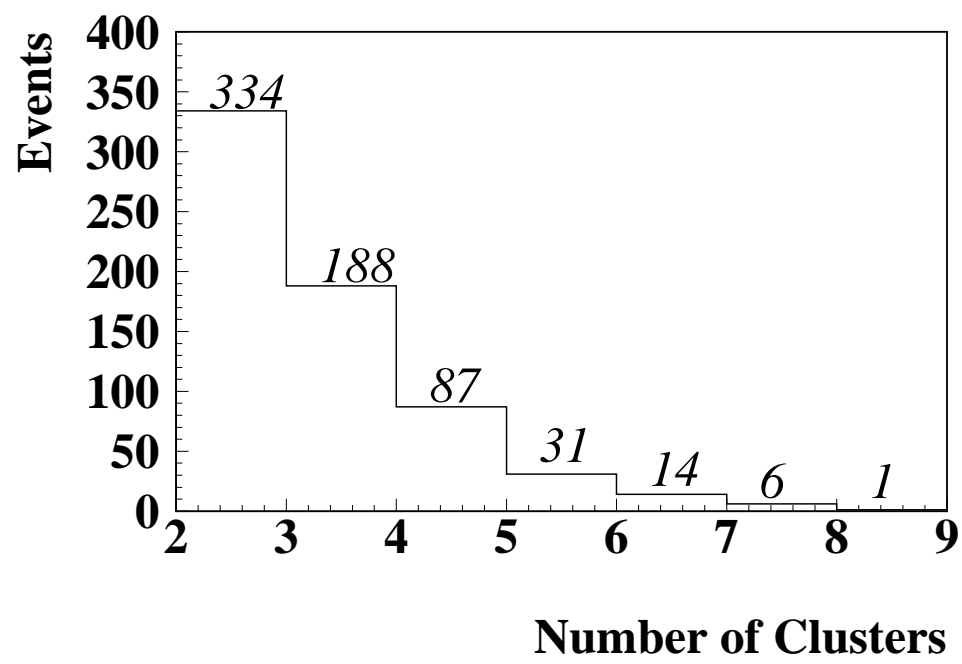

Figure 7.3: Number of CCAL clusters for events (taken from four runs near the $\eta_{c}$ peak) which pass all the cuts discussed in this chapter, except the timing cut.

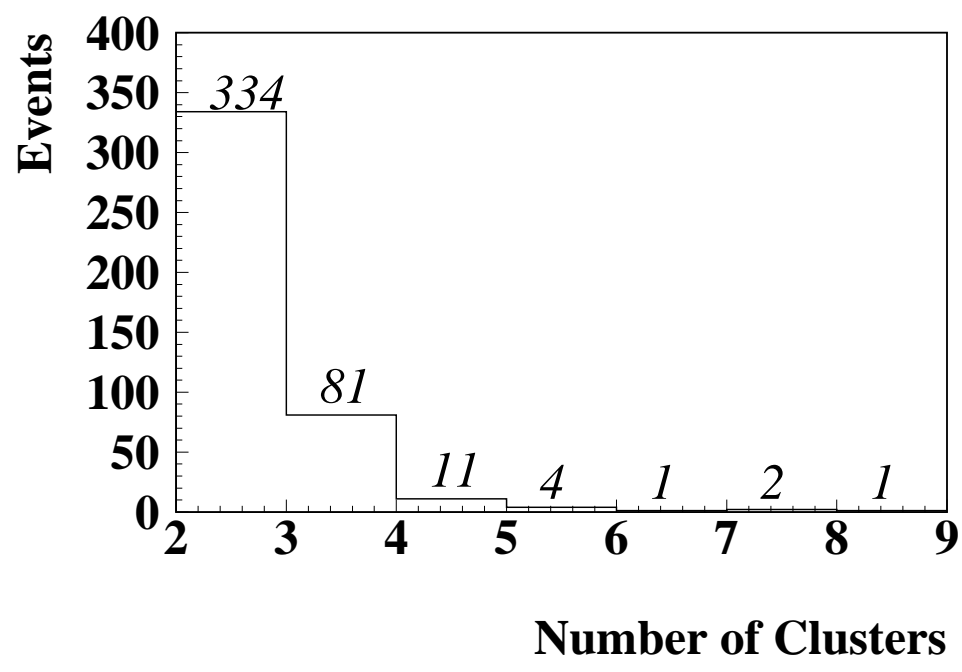

Figure 7.4: Number of CCAL clusters for events (taken from four runs near the $\eta_{c}$ peak) which pass all cuts discussed in this chapter. 
Table 7.1: $\pi^{0} \pi^{0}\left(\pi^{0} \gamma\right)$ events with exactly 4(3) CCAL clusters, with $N_{\text {on-time }}$ having an in-time TDC signal. Note the high rejection factor for $\pi^{0} \pi^{0}$ and $\pi^{0} \gamma$ events if $N_{\text {in-time }}=2$ is used.

\begin{tabular}{|c|c|c|c|c|c|c|c|c|c|}
\hline \multirow[b]{2}{*}{ Region } & \multirow{2}{*}{$\begin{array}{r}\pi^{0} \pi^{0} \\
\text { Events }\end{array}$} & \multicolumn{3}{|c|}{$\mathrm{N}_{\text {in-time }}$} & \multirow{2}{*}{$\begin{array}{r}\text { Rejection } \\
\text { Factor }\end{array}$} & \multirow{2}{*}{$\begin{array}{r}\pi^{0} \gamma \\
\text { Events }\end{array}$} & \multicolumn{2}{|c|}{$\mathrm{N}_{\text {in-time }}$} & \multirow{2}{*}{$\begin{array}{r}\text { Rejection } \\
\text { Factor }\end{array}$} \\
\hline & & 4 & 3 & 2 & & & 3 & 2 & \\
\hline$\eta_{c}$ & 12538 & 11846 & 683 & 9 & 0.9993 & 16470 & 16127 & 343 & 0.9792 \\
\hline${ }^{1} P_{1}$ & 5076 & 4815 & 260 & 1 & 0.9998 & 6509 & 6346 & 163 & 0.9750 \\
\hline$\eta_{c}^{\prime}$ & 2563 & 2435 & 127 & 1 & 0.9996 & 3120 & 3029 & 91 & 0.9708 \\
\hline
\end{tabular}

centage of fully-accepted $\pi^{0} \gamma$ and $\pi^{0} \pi^{0}$ events. An examination of the number of on-time clusters for a clean selection of $\pi^{0} \gamma$ and $\pi^{0} \pi^{0}$ events demonstrates this fact.

For this study, $\pi^{0} \pi^{0}$ events were chosen by requiring that there be precisely four clusters in CCAL and that two invariant masses $M\left(\gamma_{1,2} \gamma\right)$ in the range 115-155 $\mathrm{MeV}$ be found. $\pi^{0} \gamma$ events were selected in a similar way, requiring one $M\left(\gamma_{1,2} \gamma\right)$ combination in the same range, and exactly three CCAL clusters. The number of events having $N_{\text {in-time }}$ in-time clusters for each of these cases can be found in Table 7.1, for data taken in the vicinity of $\eta_{c}$ and $\chi_{2}$, and in the search region for $\eta_{c}^{\prime}$. We note that when the $N_{\text {in-time }}=2$ cut alone is applied, less than $\sim 0.07 \%$ of the $\pi^{0} \pi^{0}$ events, and less than $\sim 2.5 \%$ of the $\pi^{0} \gamma$ events, are retained. As an aside, we note that $\sim 5 \%$ of the $\pi^{0} \pi^{0}$ events have only 3 in-time clusters and that $\sim 2.5 \%$ of the $\pi^{0} \gamma$ events have only two in-time clusters.

The $N_{\text {in-time }}=2$ cut will be inefficient for selecting $\gamma \gamma$ final states only if additional interactions occurring within \pm 10 ns of the trigger interaction cause extra in-time hits, or if random noise produces a cluster with an in-time TDC hit. The inefficiency induced by each of these situations may be dependent on the instanta- 
neous luminosity, and therefore the efficiency of the timing cut has been studied in conjunction with other cuts which are expected to be luminosity dependent. The study of the combined efficiency of such cuts is described in Sec. 7.2.4.

\subsubsection{Invariant mass between $\gamma$ candidate cluster and "ex- tra" clusters}

After applying the $N_{\text {in-time }}=2$ cut, remaining events contain either the 2 in-time clusters alone, or in addition, some number of out-of-time or untimed clusters. Lower energy clusters (below $50 \mathrm{MeV}$ or so) have a $\gtrsim 20 \%$ chance of being untimed, as illustrated in Fig. 7.5. Because of this drop in timing efficiency with decreasing cluster energy, it is possible that a real $\pi^{0} \gamma$ event, for instance, may be observed in the CCAL as a pair of $\gamma$ candidates accompanied by a third cluster which is either untimed or out-of-time. Such events are removed as described below.

We calculate invariant masses $M\left(\gamma_{1,2} \gamma\right)$ between all untimed or out-of-time clusters and each of the $\gamma$ candidates, $\gamma_{1}$ and $\gamma_{2}$. We reject events which have one or more invariant mass combinations in the $\pi^{0}$ mass range $100 \leq M\left(\gamma_{1,2} \gamma\right)(\mathrm{MeV}) \leq 170$. The invariant mass spectrum of the pairs $\left(\gamma_{1}, \gamma\right.$ and $\left.\gamma_{2}, \gamma\right)$ is shown in Fig. 7.6, and the cut region is indicated.

In the E760 analyses of the $\gamma \gamma$ final state, $[48,49]$ events having a combination of $M\left(\gamma_{1,2} \gamma\right)$ near the $\eta$ mass $(549 \mathrm{MeV})$ were also removed. We do not make such a cut in E835, however, because the CCAL timing capability makes the cut unnecessary. While the lower energy photon from $\pi^{0}$ decay (in a $\pi^{0} \pi^{0}$ or $\pi^{0} \gamma$ event) may sometimes have a wrongly assigned time, resulting in an untimed or out-of-time cluster, in the

case of $\eta$ 's this happens rarely, if ever. The absence of a discernible excess in the 


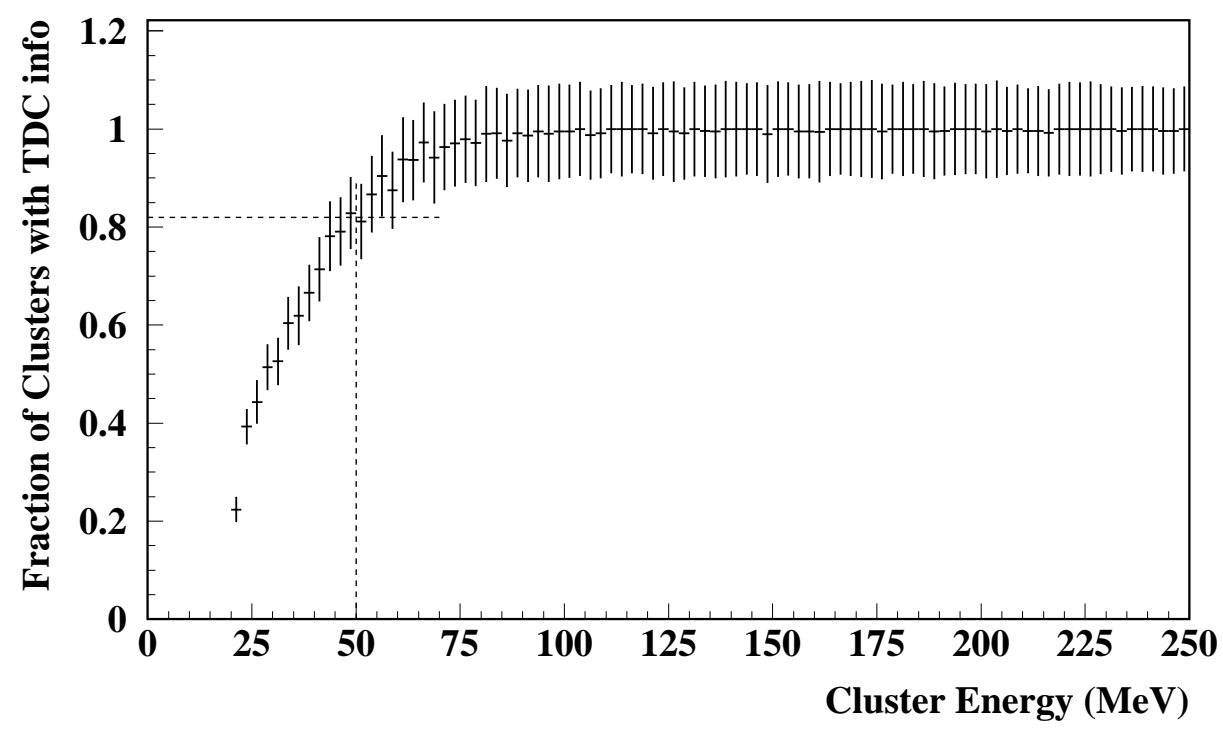

Figure 7.5: Fraction of clusters which have timing information. These clusters were chosen from $\pi^{0} \pi^{0}$ events, in which all four clusters are reconstructed in the CCAL, and therefore should in principle have in-time signals. The dashed lines indicate, e.g., that $\sim 82 \%$ of $50 \mathrm{MeV}$ clusters have correct timing information.

$M\left(\gamma_{1,2} \gamma\right)$ spectrum near $550 \mathrm{MeV}$ in Fig. 7.6, is testimony to this fact.

The efficiency of this cut has been studied in combination with other cuts which have a possible luminosity dependence, and the results of that study are reported in Sec. 7.2.4.

\subsubsection{Kinematic cuts}

Next, the $\gamma \gamma$ candidate events are evaluated for consistency with the kinematics of $\bar{p} p \rightarrow \gamma \gamma$. We have investigated two ways of doing this. First, a $4 \mathrm{C}$ kinematic fit may 


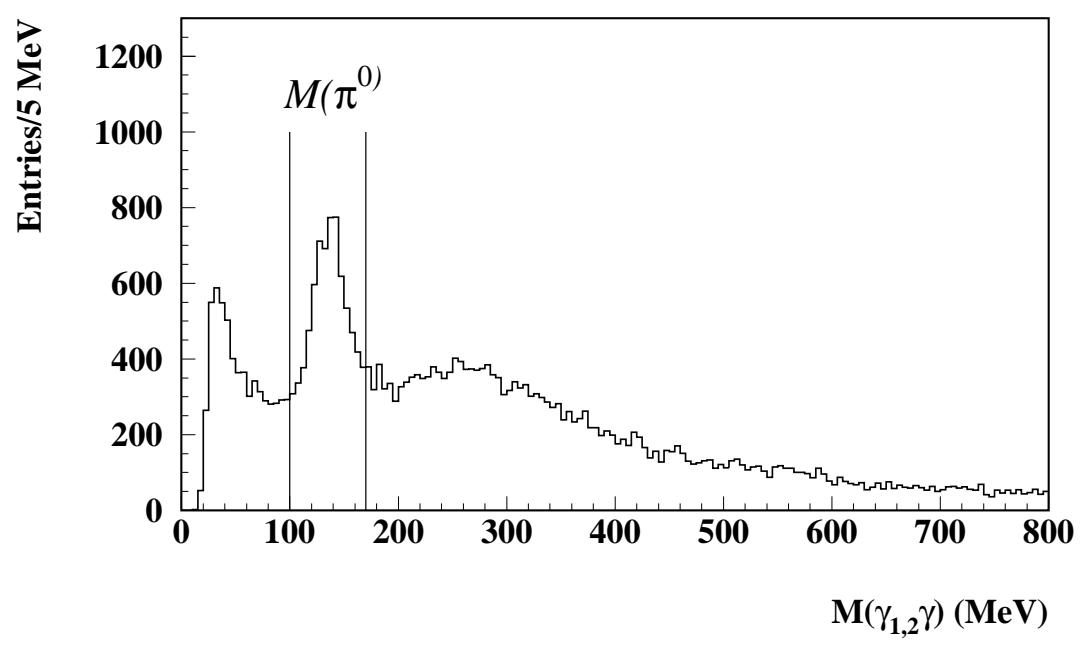

Figure 7.6: Invariant mass spectrum: $M\left(\gamma_{1,2} \gamma\right)$ between $\gamma$ candidates and any untimed or out-of-time clusters in the CCAL. This spectrum was produced with data taken from runs in the $\eta_{c}^{\prime}$ region.

be performed on the two $\gamma$ candidates, from which a $\chi^{2}$ probability may be calculated and cut on. Second, separate cuts on the variables $\Delta \theta=\left(\theta_{\text {measured }}-\theta_{\text {expected }}\right)$ and $\Delta \phi=\left(\left|\phi_{1}-\phi_{2}\right|-\pi\right)$ may be made. In the analysis presented in this dissertation, we have chosen to use the first method, though the second method is an entirely proper alternative. We explain our choice in the following.

Cuts on $\chi^{2}$ Probability: The $\chi^{2}$ probability for a clean selection of $J / \psi \rightarrow e^{+} e^{-}$ events (chosen from the "gold ee" trigger set, requiring only 2 clusters in the CCAL, and pulse heights in the Cerenkov of 0.5-1.5 units for each electron candidate) is shown in Fig. 7.7. Our chosen $\chi^{2}$ probability cut of $5 \%$ is indicated in the figure 


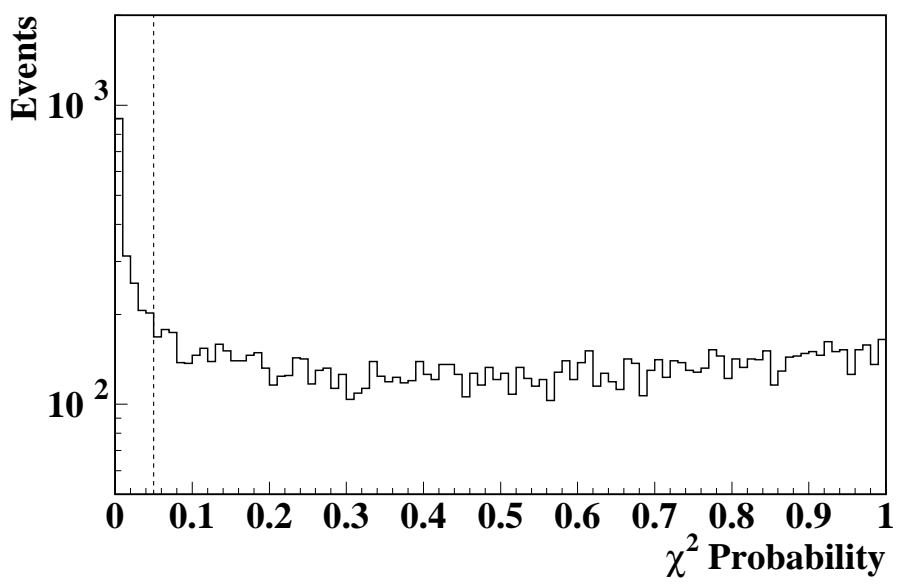

Figure 7.7: $\chi^{2}$ probability for clean $J / \psi \rightarrow e^{+} e^{-}$events. The dashed line indicates our chosen cut value of $5 \%$.

by the dashed line. We have estimated the efficiency of this cut using the clean sample of $J / \psi \rightarrow e^{+} e^{-}$events from our data. The efficiency for various values of the $\chi^{2}$ probability cut is shown in Table 7.2.

In an effort to check the stability of this cut throughout the E835 run, we have calculated the efficiency of the cut for individual $J / \psi$ stacks, and the result is shown in Table 7.2 as well. This efficiency appears to be quite stable throughout the run, though the last set of runs (3078-3079) indicates a possible problem. There are, however, no known problems with the CCAL or instantaneous luminosity for this set of runs. Other consistency checks of the CCAL do not exhibit this problem; so we conservatively estimate a systematic error of $1 \%$, so that the efficiency of the cut at $\chi^{2}$ probability $\leq 5 \%$ as determined by the $J / \psi$ data sample is $87.30 \pm 0.25 \pm 1.00 \%=$ $87.3 \pm 1.03 \%$. 
Table 7.2: Efficiencies of various $\chi^{2}$ probability cuts for $J / \psi \rightarrow e^{+} e^{-}$events.

\begin{tabular}{|l|l|}
\hline $\begin{array}{l}\text { Probability } \\
\text { Cut level }\end{array}$ & $\begin{array}{l}\text { Efficiency }(\%) \\
\text { at } \mathrm{J} / \psi\end{array}$ \\
\hline $0.5 \%$ & $95.20 \pm 0.16$ \\
$1 \%$ & $93.84 \pm 0.18$ \\
$5 \%$ & $87.30 \pm 0.25$ \\
$10 \%$ & $81.92 \pm 0.29$ \\
\hline
\end{tabular}

\begin{tabular}{|l|l|}
\hline \multicolumn{2}{|c|}{ Probability cut at 5\% } \\
\hline Runs & Efficiency (\%) \\
\hline $824-831$ & $87.49 \pm 0.49$ \\
$908-909$ & $87.81 \pm 0.49$ \\
$1331-1336$ & $87.20 \pm 0.52$ \\
$2285-2286$ & $88.02 \pm 0.56$ \\
$3078-3079$ & $83.82 \pm 0.96$ \\
\hline
\end{tabular}
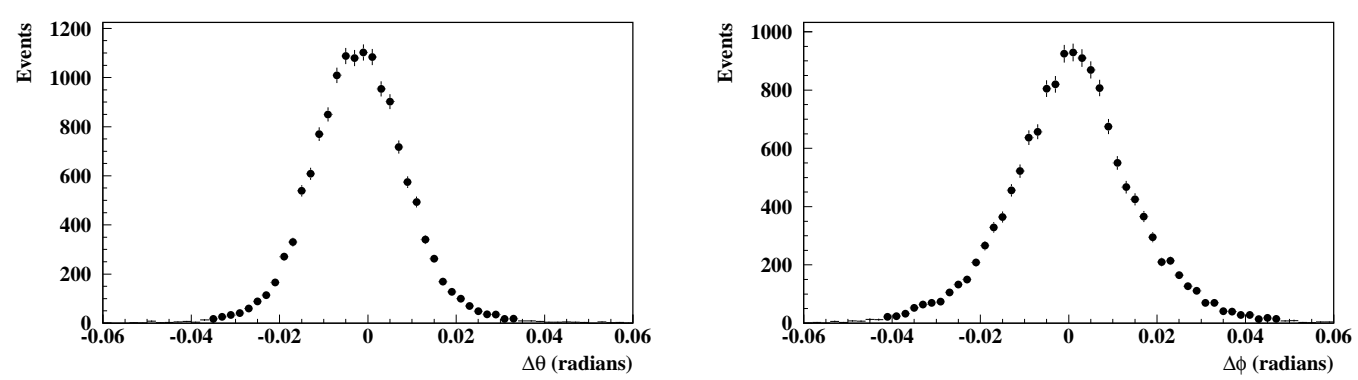

Figure 7.8: $\Delta \theta$ (left) and $\Delta \phi$ (right) distributions for clean $J / \psi \rightarrow e^{+} e^{-}$events..

Cuts on $\Delta \theta$ and $\Delta \phi:$ In an effort to determine whether a different set of cuts on two-body kinematics of the $\gamma$ candidates are useful, we have studied the efficiency of cuts on $\Delta \theta$ and $\Delta \phi$. The $\Delta \theta$ and $\Delta \phi$ distributions for the set of clean $J / \psi$ events described earlier are shown in Fig. 7.8.

The mean values of $\Delta \theta$ and $\Delta \phi$ are not zero, but change somewhat from stack to stack, or even from run to run. We therefore consider cuts which are dependent on the difference between $\Delta \theta(\Delta \phi)$ and the mean value, $\overline{\Delta \theta}(\overline{\Delta \phi})$. We refer to these cuts as cuts on the 'rescaled' variables $\Delta_{R}(\theta)=|\Delta \theta-\overline{\Delta \theta}|$ and $\Delta_{R}(\phi)=|\Delta \phi-\overline{\Delta \phi}|$. The efficiencies for various cut values of rescaled $\Delta_{R}(\theta)$ and $\Delta_{R}(\phi)$ are given in 
Table 7.3: Efficiencies of $\Delta_{R}(\theta)$ and $\Delta_{R}(\phi)$ cuts. (expressed in \%)

\begin{tabular}{|l||l|l|l|}
\hline \multicolumn{1}{|c||}{} & \multicolumn{3}{c|}{$\Delta_{R} \phi$} \\
\hline$\Delta_{R} \theta$ & 0.015 & 0.020 & 0.025 \\
\hline 0.015 & $62.36 \pm 0.36$ & $71.86 \pm 0.34$ & $77.33 \pm 0.31$ \\
0.020 & $68.45 \pm 0.35$ & $78.92 \pm 0.30$ & $85.00 \pm 0.27$ \\
0.025 & $71.00 \pm 0.34$ & $81.87 \pm 0.29$ & $88.20 \pm 0.24$ \\
0.030 & $72.15 \pm 0.33$ & $83.23 \pm 0.29$ & $89.68 \pm 0.23$ \\
\hline & \multicolumn{3}{|c|}{$\Delta_{R} \phi$} \\
\hline \hline$\Delta_{R} \theta$ & 0.027 & 0.030 & 0.035 \\
\hline 0.015 & $78.87 \pm 0.30$ & $80.63 \pm 0.29$ & $82.39 \pm 0.28$ \\
0.020 & $86.64 \pm 0.25$ & $88.63 \pm 0.24$ & $90.61 \pm 0.22$ \\
0.025 & $89.96 \pm 0.22$ & $92.05 \pm 0.20$ & $94.10 \pm 0.18$ \\
0.030 & $91.46 \pm 0.21$ & $93.59 \pm 0.18$ & $95.67 \pm 0.15$ \\
\hline
\end{tabular}

\section{Table 7.3}

As a test for the stack-to-stack stability of this cut (to be compared with that of the $\chi^{2}$ cut discussed previously), we chose values of $\Delta_{R}(\theta)$ and $\Delta_{R}(\phi)$ for which the cut efficiency is approximately the same $(\sim 87 \%)$ as for a $\chi^{2}$ cut at $5 \%$. One set of these values is $\Delta_{R}(\theta)=0.020$ and $\Delta_{R}(\phi)=0.027$. The efficiencies of this cut for each of the four $J / \psi$ stacks considered earlier are given in Table 7.4. As is apparent from the table, this cut may be more stable from stack to stack, and its use therefore would lead to a somewhat smaller systematic error $(\sim 0.5 \%)$.

However, while the probability cut and the $\Delta_{R}(\theta), \Delta_{R}(\phi)$ cuts are nearly equally efficient for $\bar{p} p \rightarrow J / \psi \rightarrow e^{+} e^{-}$, their ability to reject background is not equal. We have studied the background level accepted by each cut, and find that the cut on $\chi^{2}$ probability is more efficient at removing background. For example, for a set of ten ${ }^{1} P_{1}$ stacks, where we expect no $\gamma \gamma$ signal, we have applied the two kinematic cuts to events which pass all other cuts mentioned in this chapter. Of these 'background' 
Table 7.4: Efficiencies of $\Delta_{R}(\theta)$ cut at 0.020 and $\Delta_{R}(\phi)$ at 0.027 for various $J / \psi$ runs.

\begin{tabular}{|l|l|}
\hline \hline Runs & Efficiency (\%) \\
\hline \hline $824-831$ & $86.43 \pm 0.51$ \\
$908-909$ & $87.22 \pm 0.50$ \\
$1331-1336$ & $86.58 \pm 0.53$ \\
$2285-2288$ & $86.36 \pm 0.59$ \\
$3078-3079$ & $86.39 \pm 0.89$ \\
\hline
\end{tabular}

events, the $\Delta_{R}(\theta)$ and $\Delta_{R}(\phi)$ cuts reject $\sim 60 \%$ while the $\chi^{2}$ probability cut rejects $\sim 70 \%$.

We have also considered six other combinations of $\Delta_{R}(\theta)$ and $\Delta_{R}(\phi)$ which have approximately the same efficiency as the choice of $\Delta_{R}(\theta)=0.02$ and $\Delta_{R}(\phi)=0.027$ which we studied above. They all are found to have nearly the same background rejection efficiency. We have therefore decided to use the $\chi^{2}$ probability cut.

\subsubsection{Effect of "Noise" and Extraneous Interactions on Ef- ficiency}

As instantaneous luminosity varies, the efficiencies of several cuts mentioned above may change. The rate of interactions is proportional to instantaneous luminosity - and therefore, the occurrence of clusters in the CCAL due to interactions other than a triggered event increases as the instantaneous luminosity is increased. The rate of false neutral vetoes increases in a similar manner. Further, random noise in the CCAL and other detectors may also be stack-dependent.

The occurrence of each of these problems is best studied by using Monte Carlo $\gamma \gamma$ events superimposed with events from the random gate trigger. For each E835 
stack, a Monte Carlo set of 100,000 $\gamma \gamma$ events is generated. Then, for each Monte Carlo event, CCAL and hodoscope information from a random gate event is added to the information which has been simulated by Monte Carlo. In this way, the activity of the detector, due either to random noise or additional interactions during the same FERA gate, are simulated.

By this technique it is possible to estimate the fraction of real $\gamma \gamma$ events which are removed from the data set due to

1. random noise which generates extra in-time clusters,

2. $m\left(\pi^{0}\right)$ combinations between the $\gamma$ candidates and clusters due to random noise or out-of-time interactions, or

3. a false Neutral Veto due to noise, or a coincident charged interaction.

This study was performed for every E835 stack. The efficiencies calculated for the combination of the cuts on timing, $M\left(\gamma_{1,2} \gamma\right)$ and the $\chi^{2}$ probability, and the inefficiency for the neutral veto, are shown in Fig. 7.9 as a function of the instantaneous luminosity. These data have been fitted to a line:

$$
\epsilon_{L D}=(0.947 \pm 0.002)-(0.109 \pm 0.001) \times \mathcal{L}
$$

where the instantaneous luminosity $\mathcal{L}$ is expressed in units of $10^{31} \mathrm{~cm}^{-2} \mathrm{sec}^{-1}$. However, in the analysis of the $\gamma \gamma$ data, we have chosen to use the individual stack-bystack calculated efficiencies, because the fit has a large $\chi^{2} /$ d.o.f $=9.3$.

In the course this study, it was coincidentally discovered that the efficiency of the cut on $\chi^{2}$ probability has a significant energy dependence. Therefore, using a 


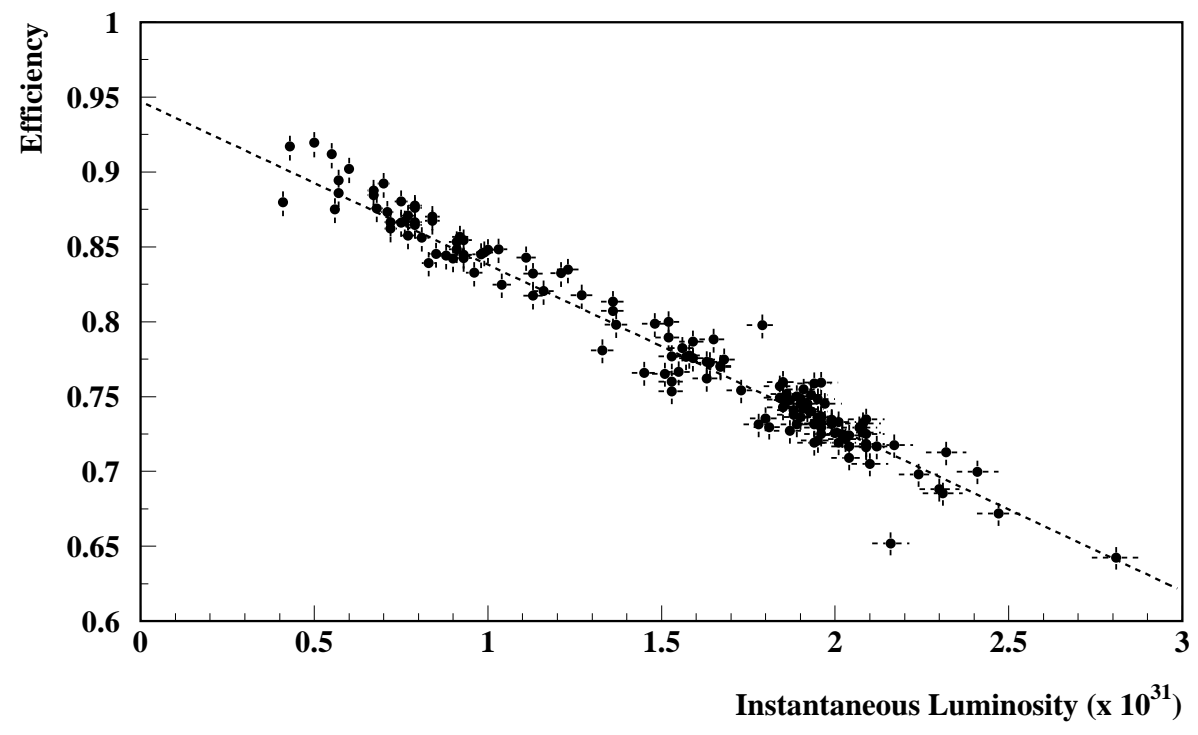

Figure 7.9: The combined efficiency of timing and $m\left(\pi^{0}\right)$ cuts, and of the neutral veto, plotted versus instantaneous luminosity. The horizontal error bars represent the $\pm 2.5 \%$ error on the luminosity measurements. The dashed line represents the results of a fit to these data.

single value for all stacks for the efficiency of cut would be incorrect. Therefore, the efficiency of this cut has also been calculated on a stack-by-stack basis. In Fig 7.10 we show the efficiency $\epsilon\left(\chi^{2}\right)$ of the $5 \% \chi^{2}$ probability cut as a function of $\sqrt{s}$. Again, these efficiencies are applied on a stack-by-stack basis in the analysis of $\gamma \gamma$ data, because the fit has a poor $\chi^{2} /$ d.o.f of 6.6 . 


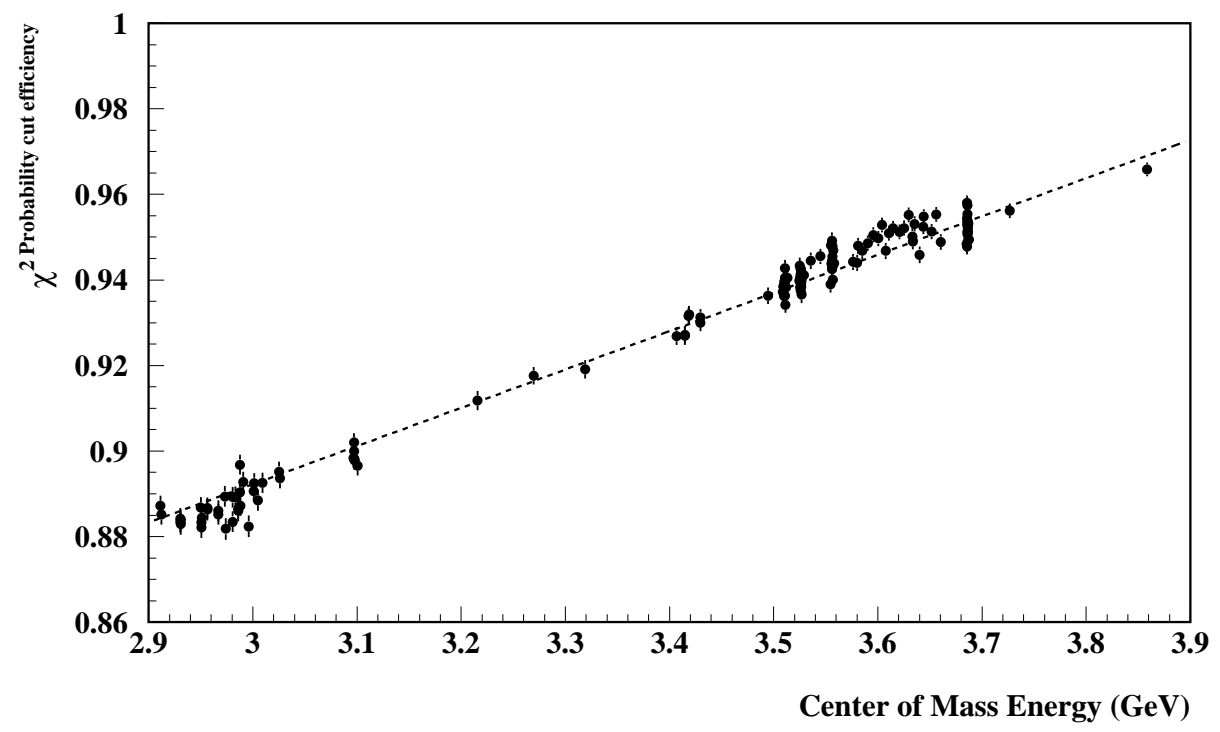

Figure 7.10: The calculated efficiency of the $5 \% \chi^{2}$ probability cut, as a function of center of mass energy $\sqrt{s}$, for events with $\left|\cos \theta^{*}\right| \leq 0.2$. The dashed line represents the results of a fit to these data.

\subsubsection{Polar Angle $\theta$ of the $\gamma$ candiates.}

As was the case in E760, the background of $\pi^{0} \pi^{0}$ and $\pi^{0} \gamma$ events which feed-down into the $\gamma \gamma$ final state is large compared to the signal. [50], [51] As a result, even after the above cuts have been made, the majority of events which remain are background events.

In order to "fake" a $\gamma \gamma$ event, two of the three photons from a $\pi^{0} \gamma$ event must take nearly all of the available energy, leaving one low energy photon which is undetected by the CCAL. Similarly from a $\pi^{0} \pi^{0}$ event which "fakes" a $\gamma \gamma$ event, two low energy photons are lost. In both cases, the two large energy photons which remain have 
essentially taken most of the energy and momentum of the parent pions; these feeddown events should therefore have an angular distribution which is essentially the same as that of the parent process.

The signal process, however, is expected to have a different angular distribution. Thus a cut on the polar angle $\theta$ of the $\gamma$ candidates can help to maximize the signal-to-background ratio.

The $\pi^{0} \pi^{0}$ and $\pi^{0} \gamma$ cross sections and the feed-down backgrounds associated with them are sharply forward peaked. In Figs. 7.11 and 7.12 we show the $\pi^{0} \pi^{0}$ and $\pi^{0} \gamma$ cross sections in the $\eta_{c}$ and $\eta_{c}^{\prime}$ regions, which demonstrate the forward peaking. In the figures we also present the $\gamma \gamma$ candidate events which have passed all cuts which we have discussed thus far. The angular distribution of those events roughly follows that of the $\pi^{0} \pi^{0}$ events as expected. We have chosen cut values of $\left|\cos \left(\theta^{*}\right)\right| \leq 0.2$ in the $\eta_{c}$ region, and $\left|\cos \left(\theta^{*}\right)\right| \leq 0.4$ at higher energies $\left(\chi_{J}, \eta_{c}^{\prime}\right)$, where the forward peaking of the feed-down background begins. These cut values are indicated in Fig. 7.11 and 7.12 .

The efficiency of this cut depends on our assumptions of the angular distribution of the process $\bar{p} p \rightarrow(c \bar{c})_{R} \rightarrow \gamma \gamma$. The pseudoscalar $\eta_{c}$ must have an angular distribution which is isotropic, thus the acceptance of the cut in the $\eta_{c}$ region is 0.2 , for an ideal detector. Similarly, for a cut at $\left|\cos \left(\theta^{*}\right)\right| \leq 0.4$, which we make for $\eta_{c}^{\prime}$, (as well as the scalar $\chi_{0}$ ) the acceptance is 0.4 . As for the $\chi_{2}$, since the $\bar{p} p \rightarrow \chi_{2} \rightarrow \gamma \gamma$ process does not have an isotropic angular distribution, the acceptance of the cut at $\left|\cos \left(\theta^{*}\right)\right| \leq 0.4$ must be calculated carefully.

The analysis of the $\bar{p} p \rightarrow \chi_{2} \rightarrow \gamma J / \psi$ reaction in E835 has shown that the $\chi_{2}$ for- 

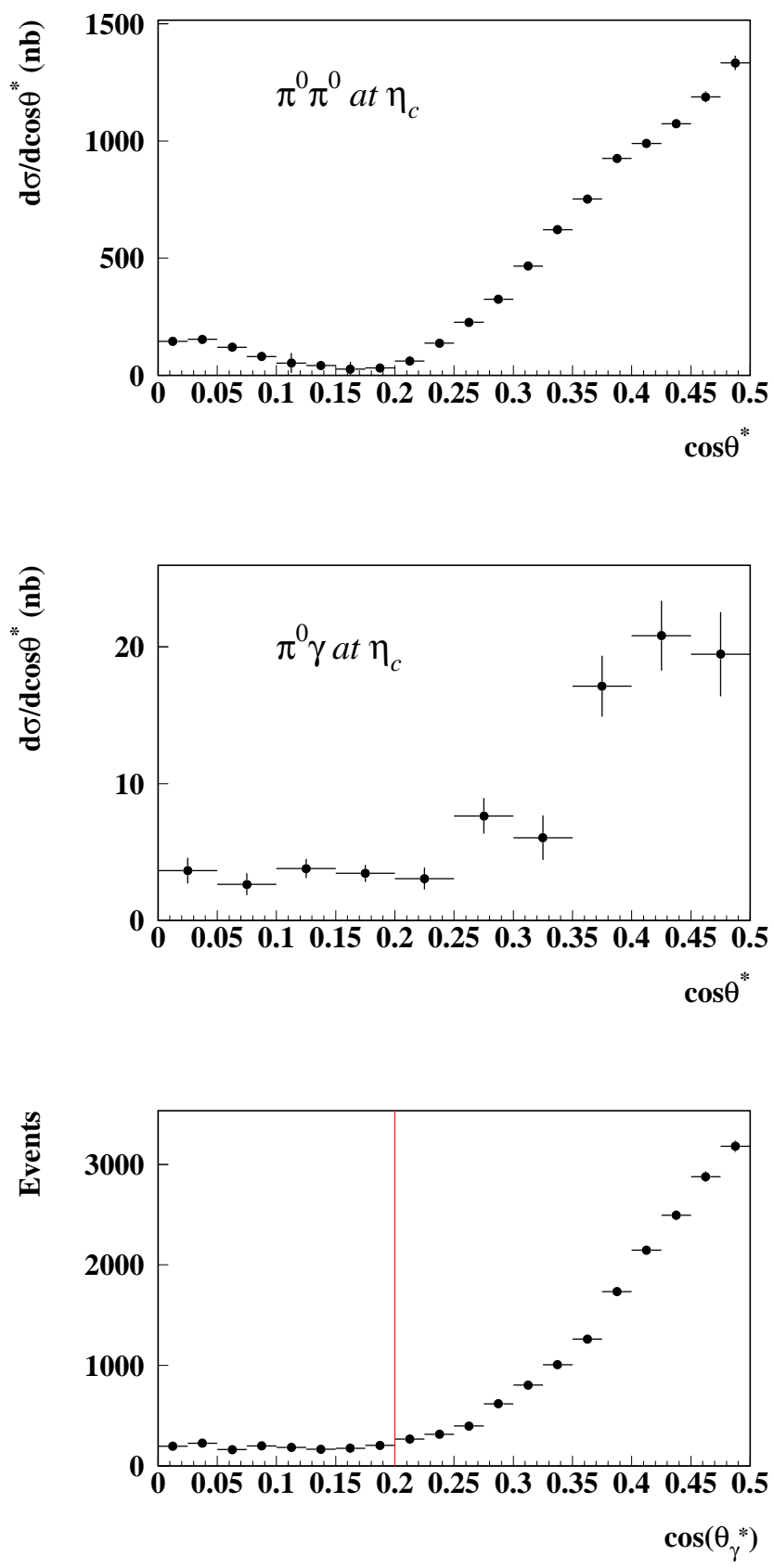

Figure 7.11: The top and middle panels show the measured $\pi^{0} \pi^{0}$ and $\pi^{0} \gamma$ angular distributions from E760 [51] at $\sqrt{s}=2985 \mathrm{MeV}$ The bottom panel shows $\gamma \gamma$ candidate events from E835 (from the whole $\eta_{c}$ region, 2911-3026 MeV) which satisfy all cuts discussed in this chapter. 

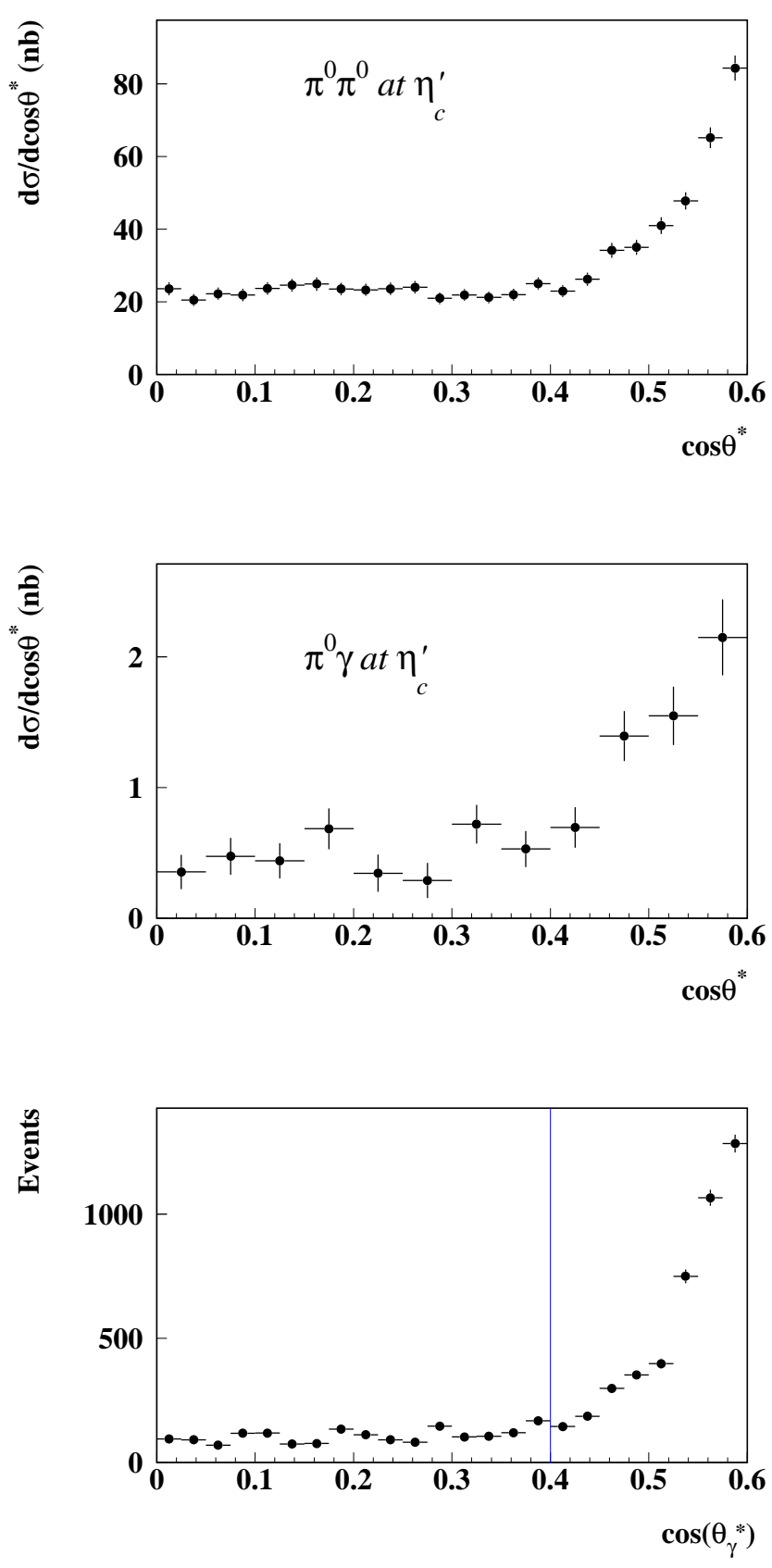

Figure 7.12: The top and middle panels show the measured $\pi^{0} \pi^{0}$ and $\pi^{0} \gamma$ angular distributions from E760 [51] at $\sqrt{s}=3619 \mathrm{MeV}$ The bottom panel shows $\gamma \gamma$ candidate events from E835 (from the ${ }^{1} P_{1}$ and $\eta_{c}^{\prime}$ regions, 3500 to $3660 \mathrm{MeV}$ ) which satisfy all cuts discussed in this chapter. 
mation is dominated by the helicity 1 state of $\bar{p} p$. An upper limit (90\% CL) of $26 \%$ was obtained for a possible helicity 0 component. [52] Further, it is theoretically predicted that in the $\chi_{2} \rightarrow \gamma \gamma$ decay the helicity 2 component should dominate. The helicity 0 component of the $\chi_{2} \rightarrow \gamma \gamma$ decay is predicted by models which take relativistic effects into account to be $\leq 5 \%$ of the partial width. [53]

Pure helicity 1 formation followed by pure helicity 2 decay gives rise to an angular distribution of the form (see Ref. [48] for a derivation)

$$
W\left(\theta^{*}\right) d \cos \theta^{*}=\frac{5}{4}\left[1-\cos ^{4} \theta^{*}\right] d \cos \theta^{*}
$$

Integration of the above function over our range of $\cos \left(\theta^{*}\right)$ from -0.4 to 0.4 gives an efficiency for $\chi_{2} \rightarrow \gamma \gamma$ of 0.497 , for an ideal detector.

Because of the fact that the efficiency of $\gamma$ detection by the CCAL is not $100 \%$, and may vary with time, we do not expect that the efficiencies for the polar angle cuts discussed above are exactly what we expect from geometrical considerations alone. We have therefore decided to evaluate the efficiency of the polar angle cuts by the Monte Carlo method described in the previous section.

\subsubsection{Charged Track Contamination at $J / \psi$ and $\psi^{\prime}$}

The kinematics of the reactions $\bar{p} p \rightarrow J / \psi \rightarrow e^{+} e^{-}$and $\bar{p} p \rightarrow \gamma \gamma$ at $\sqrt{s}=M(J / \psi)$ are indistinguishable. The same is true for the two reactions at $\psi^{\prime}$. Consequently, the events from $\bar{p} p \rightarrow\left(J / \psi, \psi^{\prime}\right) \rightarrow e^{+} e^{-}$will pass all the cuts discussed in this chapter, unless the $\gamma \gamma$ trigger used removes them effectively.

We have analyzed events from the "gold gg" trigger set, and may therefore expect 
that the number of $e^{+} e^{-}$events in the initial data sample is very small. However, if the Neutral Veto were somehow inefficient at detecting (and rejecting) $e^{+} e^{-}$events, we would expect to find an excess of events which pass our cuts at the $J / \psi$ and $\psi^{\prime}$ data points. Such a problem was discovered during the 1996 run, and as a result it is necessary to make an additional cut on charged tracks for the $\gamma \gamma$ trigger set for at least the $J / \psi$ and $\psi^{\prime}$ stacks. For consistency, we have also decided to make the same cut for data taken at all energies.

The problem involves the neutral veto logic. "Holes" were found at azimuthal $(\phi)$ angles from $(23 / 24) \pi$ to $\pi$ radians, and from $(47 / 24) \pi$ to $2 \pi$ radians. (See Fig. 7.13 ) The detector elements involved were working correctly. However, the signal cables from the $H 2^{\prime}$ paddles in the two angular regions were not correctly connected to the neutral veto logic unit. As a result, $\bar{p} p \rightarrow e^{+} e^{-}$events could pass the neutral two-body trigger in the regions of these holes. The unshaded histogram in Fig. 7.13 represents the azimuthal angle $\phi$ of each $\gamma$ candidate in events which satisfy all the cuts mentioned above. The large excess of events near $\phi=\pi$ and $\phi=2 \pi$ indicates the above-mentioned "holes" in the Neutral Veto, through which $J / \psi \rightarrow e^{+} e^{-}$events pass into the neutral trigger. In order to remove these events, we have made cuts on the pulse heights in hodoscopes $H 1$ and $H 2^{\prime}$.

It is not unexpected that, $\delta$-rays or random noise in the hodoscopes might cause a $\gamma \gamma$ event to register an pulse height of more than 0.5 MIPs in one of the four $H 1$ and $H 2^{\prime}$ paddles through which the two photons pass. On the other hand, $e^{+} e^{-}$ events can be expected to nearly always produce pulse heights of greater than 0.5 MIPs in at least three of the four paddles through which the electrons pass. For 
this reason, we remove events which have a pulse height of greater than 0.5 MIPs. in more than one of the four corresponding $H 1$ or $H 2^{\prime}$ paddles.

The shaded histogram in Fig. 7.13 shows the events which are retained after the application of this cut on $H 1$ and $H 2^{\prime}$. The excess near $\phi=0$ and $\phi=\pi$ has beeen removed.

We have tested this cut for its ability to remove $e^{+} e^{-}$events from a clean $J / \psi$ sample, and for its ability to retain clean $\pi^{0} \pi^{0}$ events, which is a test of its efficiency for our $\gamma \gamma$ event sample. From a clean sample of $5998 \mathrm{~J} / \psi \rightarrow e^{+} e^{-}$events taken from the $J / \psi$ stacks used in the $\eta_{c}$ analysis, a total of only 2 events remain after the application of the charged hodoscope cut - a $99.97 \%$ rejection factor. We have similarly studied $5.44 \mathrm{pb}^{-1}$ of data taken in the $\psi^{\prime}$ region. There, we found no $e^{+} e^{-}$ events which pass the analysis cuts, including the cut on $H 1$ and $H 2^{\prime}$ described in this section.

The Poisson 90\% confidence upper limit on 2 observed events is 5.91, and on 0 observed events, is 2.44 . Therefore, at $90 \%$ confidence we expect fewer than $5.91 \times$ $(1 / 24)=0.25 e^{+} e^{-}$events in our sample of $22 \gamma \gamma$ events at the $J / \psi$ energy points due to the presence of the holes in the trigger. Similarly, we expect $(8.98 / 5.44) \times$ $2.44 \times 1 / 24=0.17$ events in our sample of $78 \gamma \gamma$ events at the $\psi^{\prime}$ energy points. These contributions are completely negligible.

We have calculated the efficiency for $\gamma \gamma$ events using a clean sample of $9873 \pi^{0} \pi^{0}$ events at $\eta_{c}^{\prime}$. Of those, 9426 remain after the application of the $H 1$ and $H 2^{\prime}$ cuts, leading to an efficiency of $95.47 \pm 0.21 \%$. 


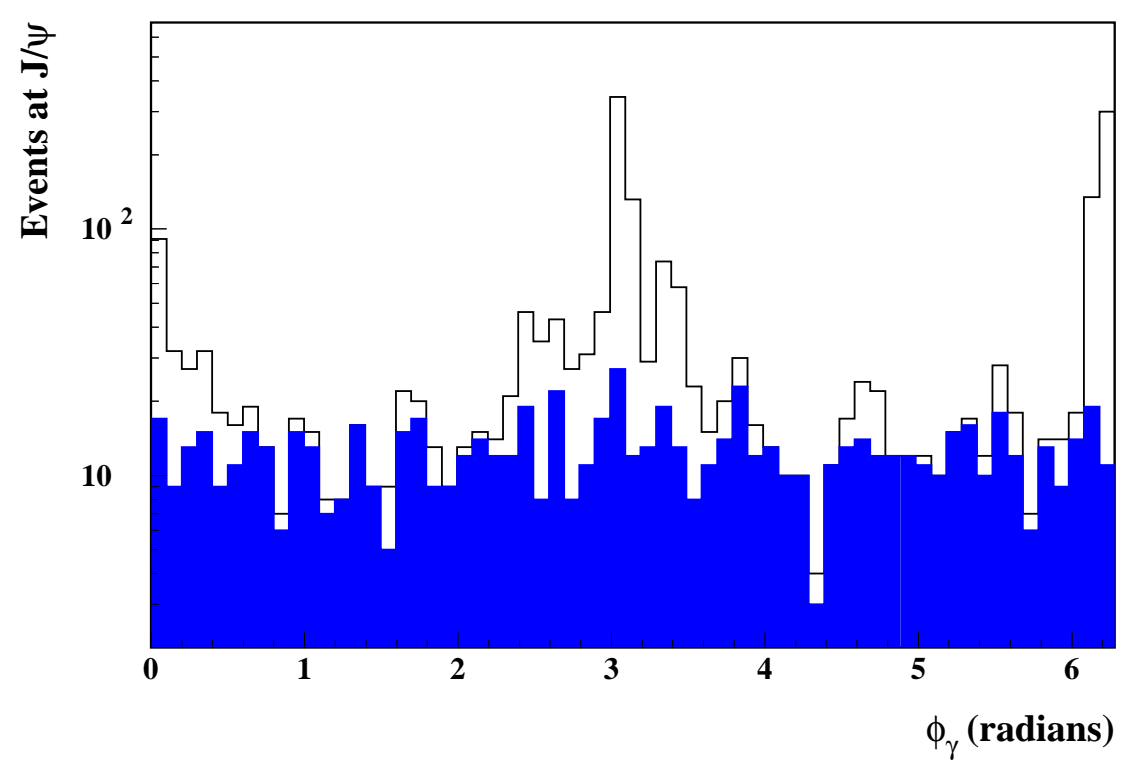

Figure 7.13: Azimuthal angle $\phi_{1,2}$ for neutral trigger events in the $J / \psi$ stacks which satisfy all cuts described in the text. The unshaded histogram shows all such events; the peaks in the distribution at $\phi=0$ and $2 \pi$ show the effect of the trigger "holes". The shaded histogram represents the events which remain after application of the $H 1$ and $H 2^{\prime}$ cuts described in the text.

\subsection{Overall Efficiency of Cuts}

The overall efficiency is determined by taking the product of the various efficiencies noted above, assuming their independence, and the $97.8 \%=1-2 \times 1.1 \%$ beampipe conversion correction derived from Ref. [47]. With the stack-by-stack efficiency described in Sec. 7.2.2 denoted as $\epsilon_{M C}$, the overall efficiency is $\epsilon=\epsilon_{M C} \times \alpha \times$ $0.934 \pm 0.020$. As mentioned above, this efficiency is calculated separately for each data point, and appears along with the summary of $\gamma \gamma$ data taken at all energy points in Table E. 


\section{Chapter 8}

\section{Results and Discussion}

In this chapter we present results for the resonance parameters of the $\eta_{c}$ and $\chi_{2}$ resonances, as well as the results of searches for $\eta_{c}^{\prime}$ and $\chi_{0}$. The data, which were collected at 146 energy points, spanning $\sqrt{s}=2911 \mathrm{MeV}$ to $4289 \mathrm{MeV}$, have been selected according to the criteria presented in Chapter 7 .

We present the cross sections for the reaction $\bar{p} p \rightarrow \gamma \gamma$ for both $\left|\cos \theta^{*}\right| \leq 0.2$ and $\left|\cos \theta^{*}\right| \leq 0.4$ in Figs. 8.1- 8.3. For clarity, data points taken at similar energies have been summed. All analyses are performed on the raw, unsummed data, however. The number of $\gamma \gamma$ candidates events, luminosity, total efficiency and cross section for each data point are tabulated in Appendix E, for each of the two choices of $\left|\cos \theta^{*}\right|$ cut.

These data show clear enhancements at $\sim 2985$ and $\sim 3556 \mathrm{MeV}$, corresponding to $\eta_{c}$ and $\chi_{2}$, respectively, and a suggestion of enhancement at $\sim 3415 \mathrm{MeV}$, corresponding to $\chi_{0}$. No clear enhancement is discernible in the region in which $\eta_{c}^{\prime}$ 


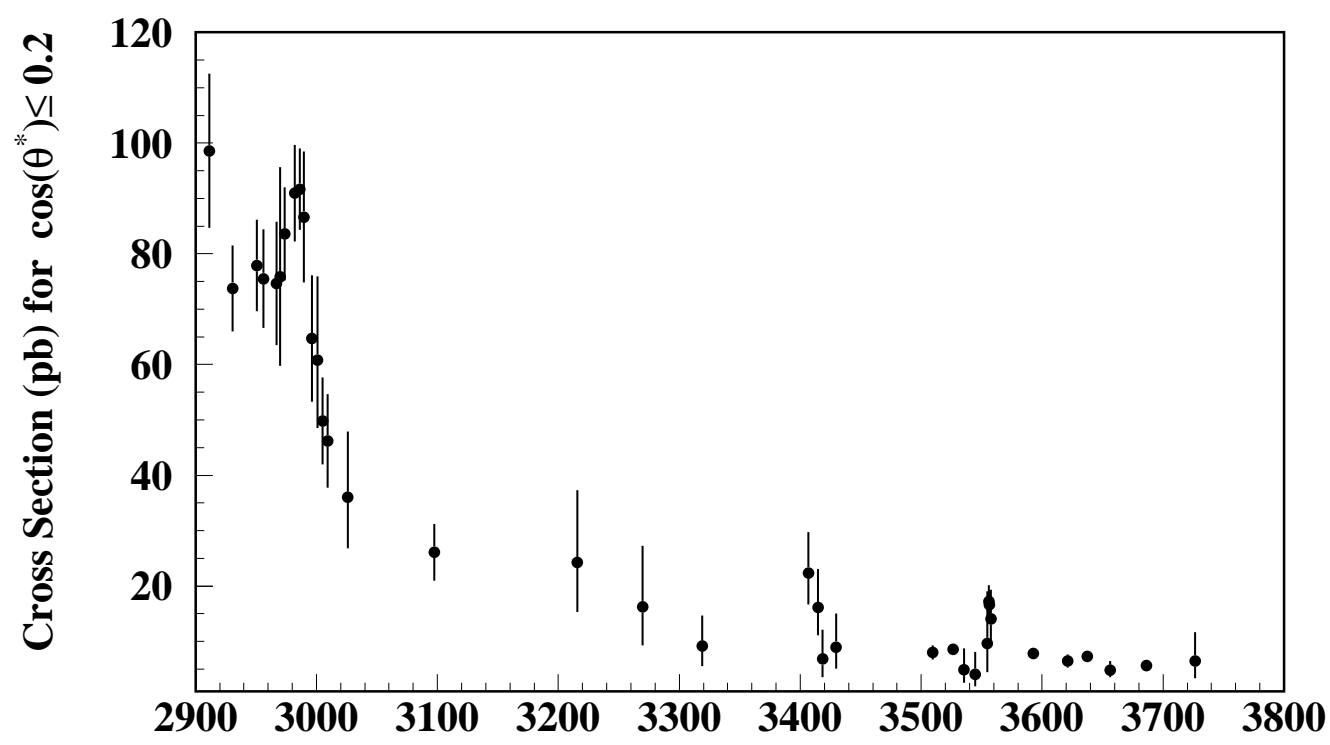

$\bigvee_{\mathrm{s}}$, Center of Mass Energy $(\mathrm{MeV})$

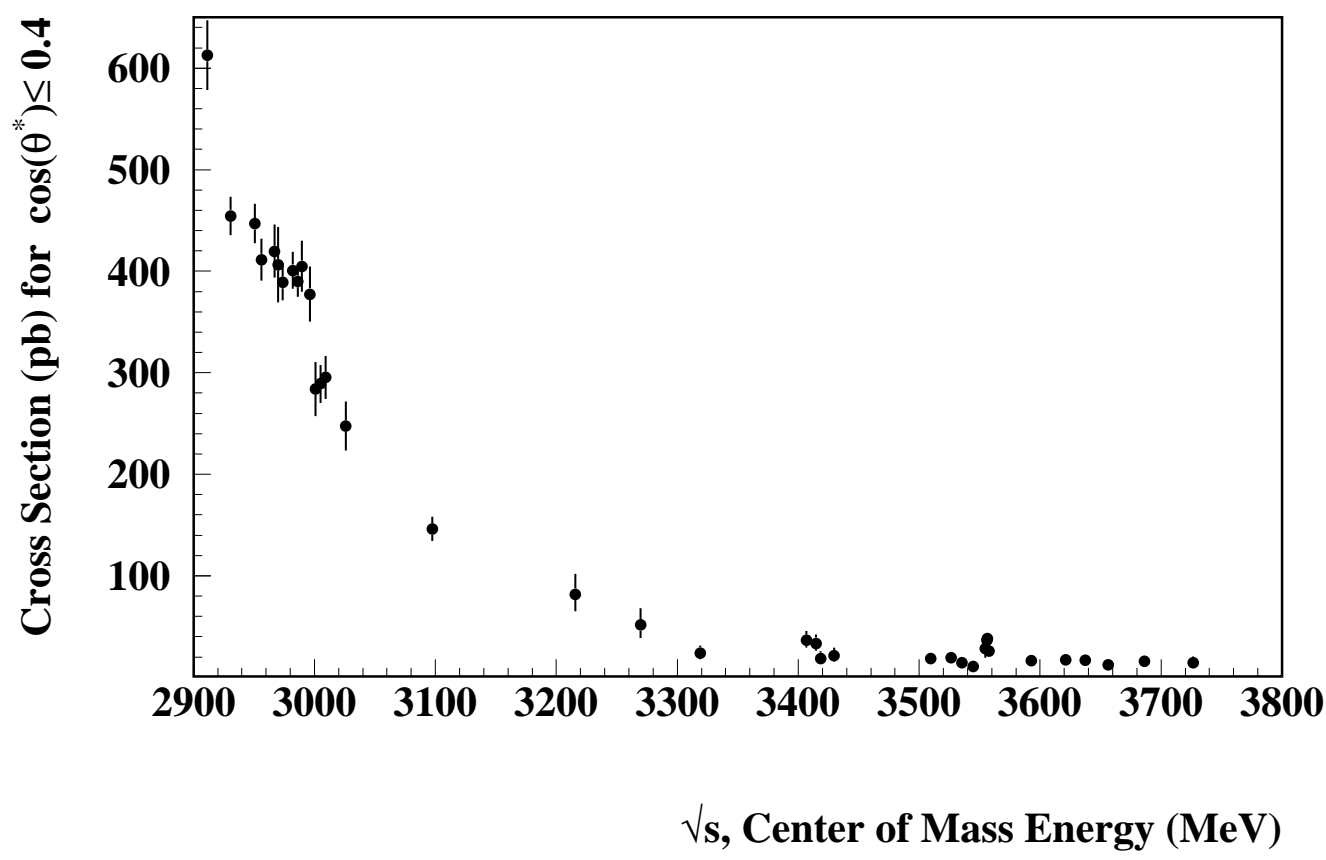

Figure 8.1: $\gamma \gamma$ Cross Sections for (top) $\left|\cos \theta^{*}\right| \leq 0.2$ and (bottom) $\left|\cos \theta^{*}\right| \leq 0.4$. 


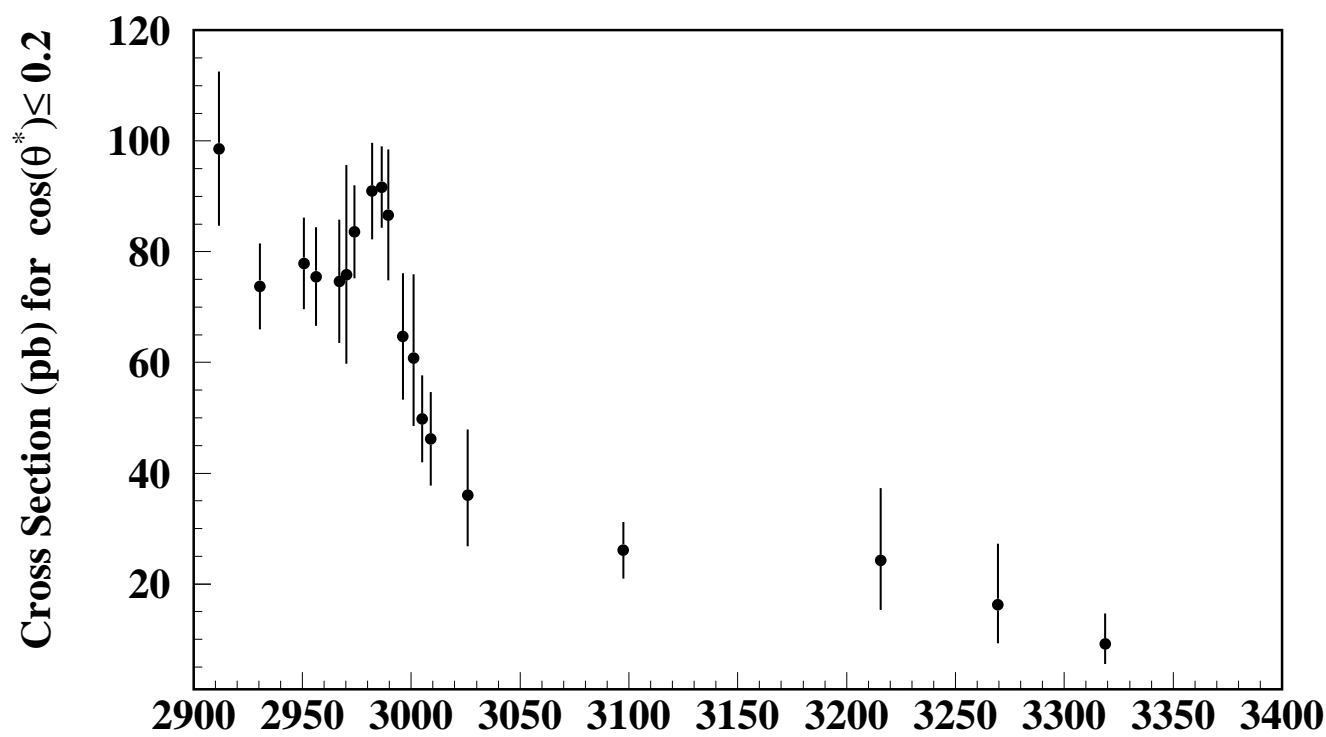

$\sqrt{s}_{\mathrm{s}}$, Center of Mass Energy $(\mathrm{MeV})$

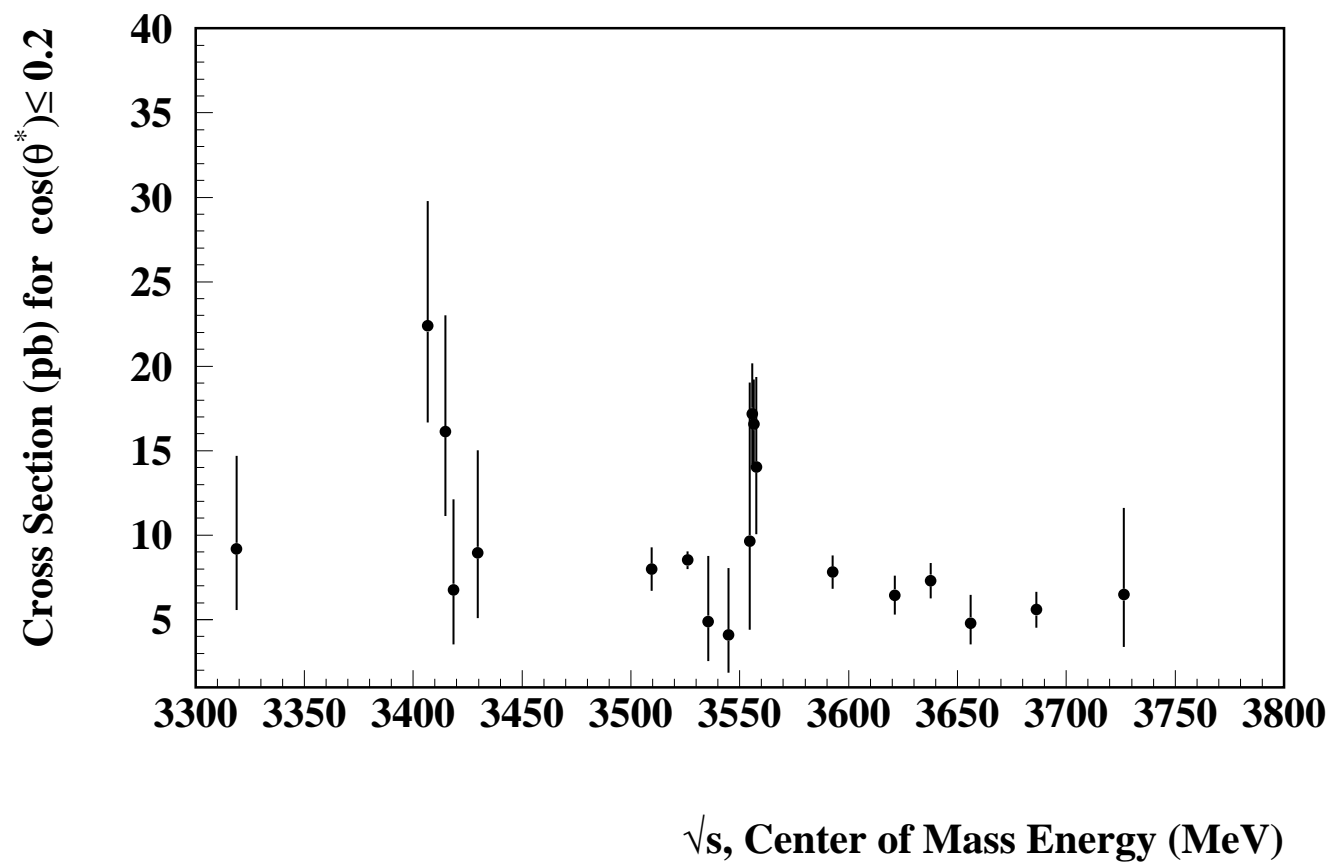

Figure 8.2: $\gamma \gamma$ Cross Sections for $\left|\cos \theta^{*}\right| \leq 0.2$, for CM energies (top) 2900-3400 $\mathrm{MeV}$ and (bottom) 3300-3800 MeV. 


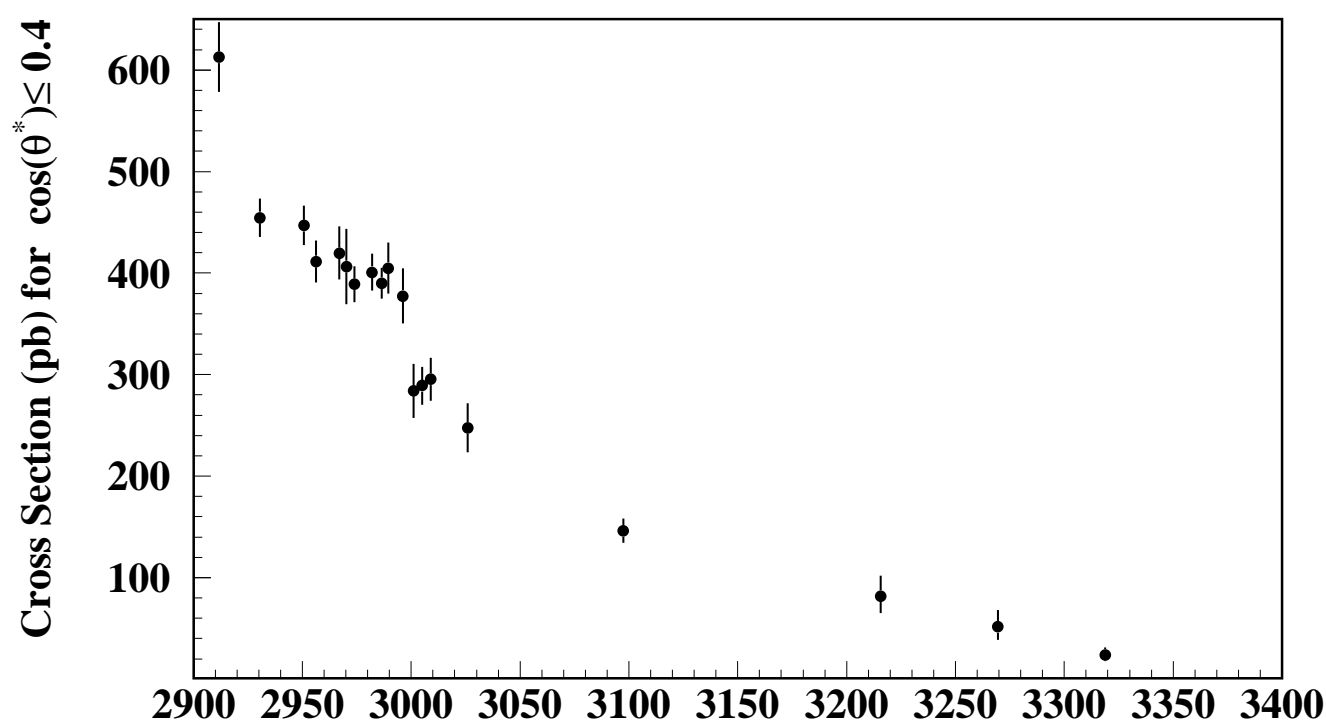

$\sqrt{ }$, Center of Mass Energy (MeV)

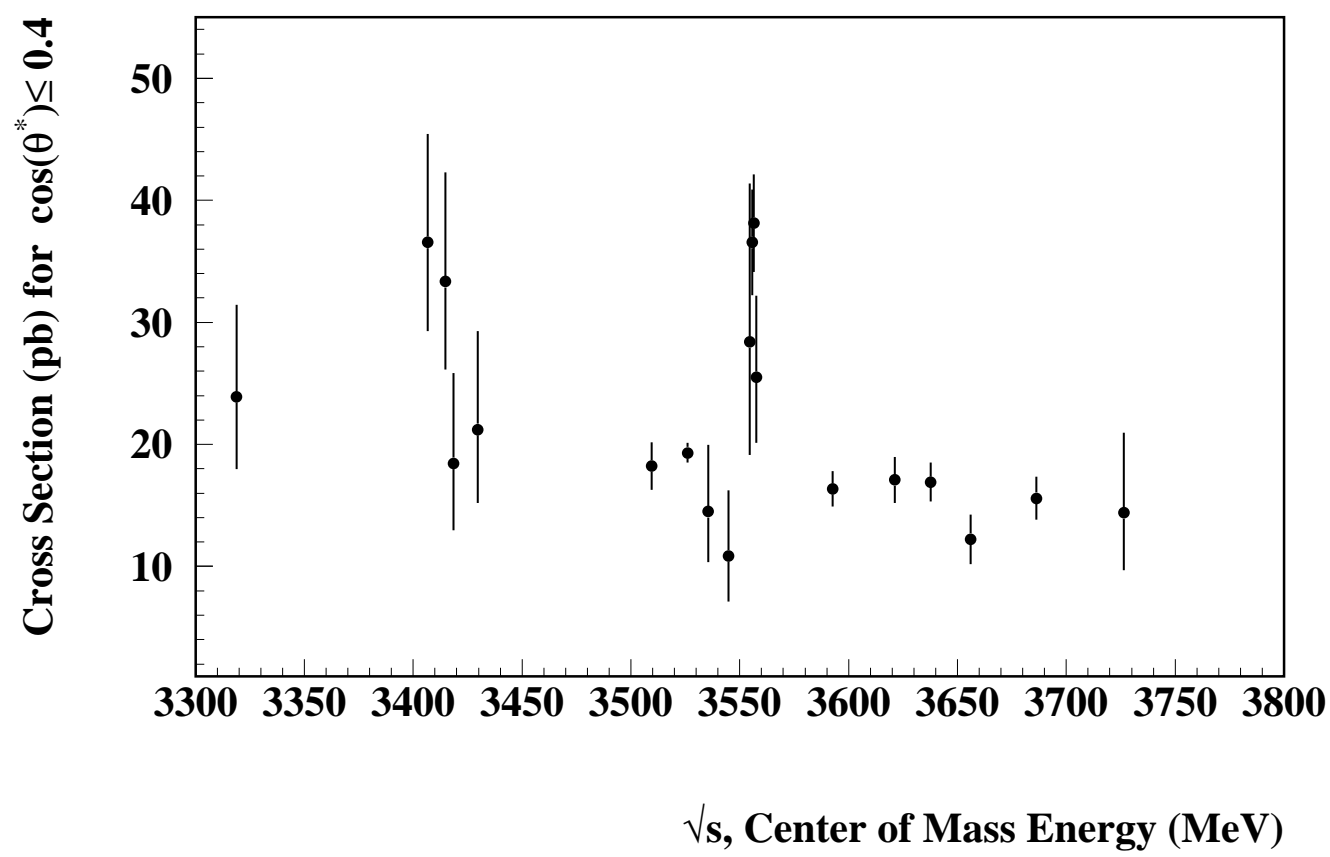

Figure 8.3: $\gamma \gamma$ Cross Sections for $\left|\cos \theta^{*}\right| \leq 0.4$, for CM energies (top) 2900-3400 $\mathrm{MeV}$ and (bottom) 3300-3800 MeV. 
is expected $(\sim 3600-3650 \mathrm{MeV})$. These figures demonstrate that even at the $\eta_{c}$ resonance, which is clearly visible, a significant amount of background remains.

\subsection{Background contributions to $(c \bar{c})_{R} \rightarrow \gamma \gamma$}

There has been much speculation as to the source of the backgrounds in the detection of the $\gamma \gamma$ final state in E760 and E835. As mentioned in Chapter 7, the majority of the background is thought to be due to $\pi^{0} \gamma$ and $\pi^{0} \pi^{0}$ events in which one or two photons, respectively, are undetected, and the events thus appear in the CCAL as a single pair of $\gamma \mathrm{s}$.

\section{The E760 Approach to the Background Problem}

E760 studied the sensitivity of the $\eta_{c}$ resonance parameters to several assumptions about the nature of the background. In all cases, the mass of $\eta_{c}$ was found to be stable at $\sim 2989 \mathrm{MeV}$. The total width of the resonance, $\Gamma\left(\eta_{c}\right)$, was found to vary between 17 and $31 \mathrm{MeV}$, and the product $B\left(\eta_{c} \rightarrow \bar{p} p\right) \times \Gamma\left(\eta_{c} \rightarrow \gamma \gamma\right)$ was found to vary between 7.7 and $9.2 \mathrm{keV}$ depending on different choices of $|\cos \theta|_{\max }$ and different assumptions about the background. In the following we review the E760 approach in order to decide whether or not to use the same method of analysis for the present data.

In E760, the background contributions to the $\gamma \gamma$ final state were estimated by means of a Monte Carlo calculation of the fraction of the measured $\pi^{0} \pi^{0}$ and $\pi^{0} \gamma$ cross sections which feed down into, or fake, the $\gamma \gamma$ channel. The resulting calcu- 


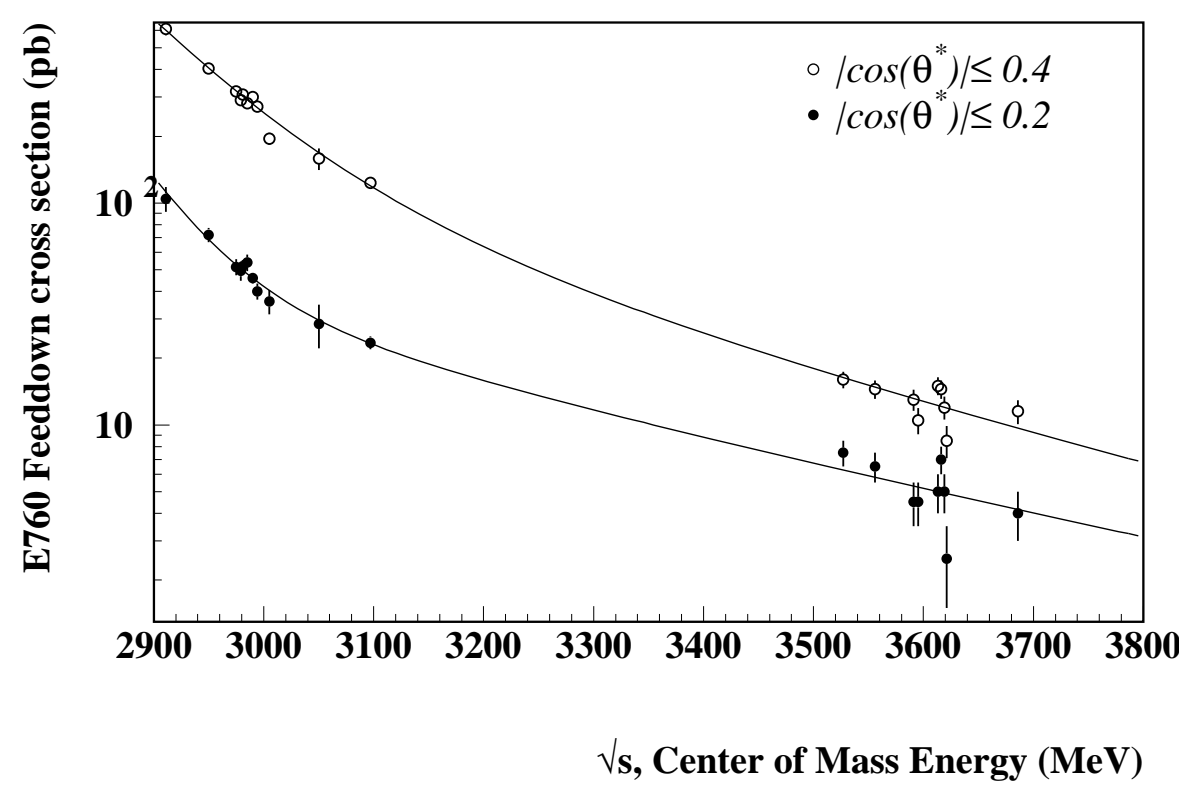

Figure 8.4: Calculated feed-down cross sections based on E760 measurements. The cross sections are calculated by multiplying the measured $\pi^{0} \pi^{0}$ and $\pi^{0} \gamma$ cross sections by the Monte Carlo generated fraction of such events which feed into the $\gamma \gamma$ channel. The data have been taken from Ref. [51]. The functions are the results of fits to the form of Eq. 8.7, which we have used to fit the E835 background to $\gamma \gamma$.

lated "feed-down" cross sections (shown in Fig. 8.4) were treated as data, with the statistical errors assumed to be the small errors in the $\pi^{0} \pi^{0}$ and $\pi^{0} \gamma$ measured cross sections. These calculated cross sections were $\sim 50 \mathrm{pb}$ and $\sim 300 \mathrm{pb}$ under the $\eta_{c}$ peak for $\left|\cos \theta^{*}\right| \leq 0.2$ and $\left|\cos \theta^{*}\right| \leq 0.4$, respectively, and $\sim 13.6$ pb under the $\chi_{2}$ peak for $\left|\cos \theta^{*}\right| \leq 0.4$.

The calculated feed-down cross sections, along with the observed $\gamma \gamma$ cross sections, were then fitted in two different regions $\left(2900-3100 \mathrm{MeV}\right.$ for $\eta_{c}$, and 3524-3686 
$\mathrm{MeV}$ for $\eta_{c}^{\prime}$ ) separately. * The cross sections were fitted to background functions of the form $A \times\left(\sqrt{s_{0}} / \sqrt{s}\right)^{B}$ plus the Breit-Wigner resonance functions. The implicit assumption in this procedure was that the feed-down calculations predicted the background accurately and completely, and that the continuum cross section $\bar{p} p \rightarrow \gamma \gamma$ was negligibly small.

E760 did, however, make an attempt to estimate possible continuum cross sections $\sigma(\bar{p} p \rightarrow \gamma \gamma)$ allowed by their data and the feed-down calculations. They concluded that $90 \%$ confidence upper limits could be set for the continuum cross sections of $21.6 \mathrm{pb}$ for $\left|\cos \theta^{*}\right| \leq 0.25$ at $\sqrt{s}=2988 \mathrm{MeV}$, and $2.3 \mathrm{pb}$ for $\left|\cos \theta^{*}\right| \leq 0.4$ at $\sqrt{s}=3600 \mathrm{MeV}$.

Experimental data for the inverse reaction, $\gamma \gamma \rightarrow \bar{p} p$ exist for $\mathrm{CM}$ energies below $3.1 \mathrm{GeV}$. The two latest measurements are those reported by CLEO [55] and VENUS [54]. These are presented in Fig. 8.5. Although the statistics of both measurements are poor in the vicinity of $3.0 \mathrm{GeV}$, no enhancement in the measured cross sections is seen in the $\eta_{c}$ vicinity, and we can safely conclude that the cross sections represent the continuum yield.

The two measurements give consistent results. At $3.0 \mathrm{GeV}$, both experiments report $\sigma(\gamma \gamma \rightarrow \bar{p} p) \approx 140 \mathrm{pb}$ integrated over the region $\left|\cos \theta^{*}\right| \leq 0.6$. Both also give enough angular distribution data to lead to the estimate that $\sigma\left(\left|\cos \theta^{*}\right| \leq\right.$ $0.2)$ is $\sim 20 \%$ of $\sigma\left(\left|\cos \theta^{*}\right| \leq 0.6\right.$ ). (see Fig. 8.6) From this we conclude that $\sigma\left(\gamma \gamma \rightarrow \bar{p} p,\left|\cos \theta^{*}\right| \leq 0.2\right)=28 \mathrm{pb}$, with an estimated error of $\leq 50 \%$. In Ref. [56],

${ }^{*}$ The analysis of the $\chi_{2} \rightarrow \gamma \gamma$ reaction in E760 was done before the feed-down technique was developed. In this analysis, the background was assumed to have the shape $A \times\left(\sqrt{s_{0}} / \sqrt{s}\right)^{B}$, but no attempt was made to calculate the background level from the possible background processes. 


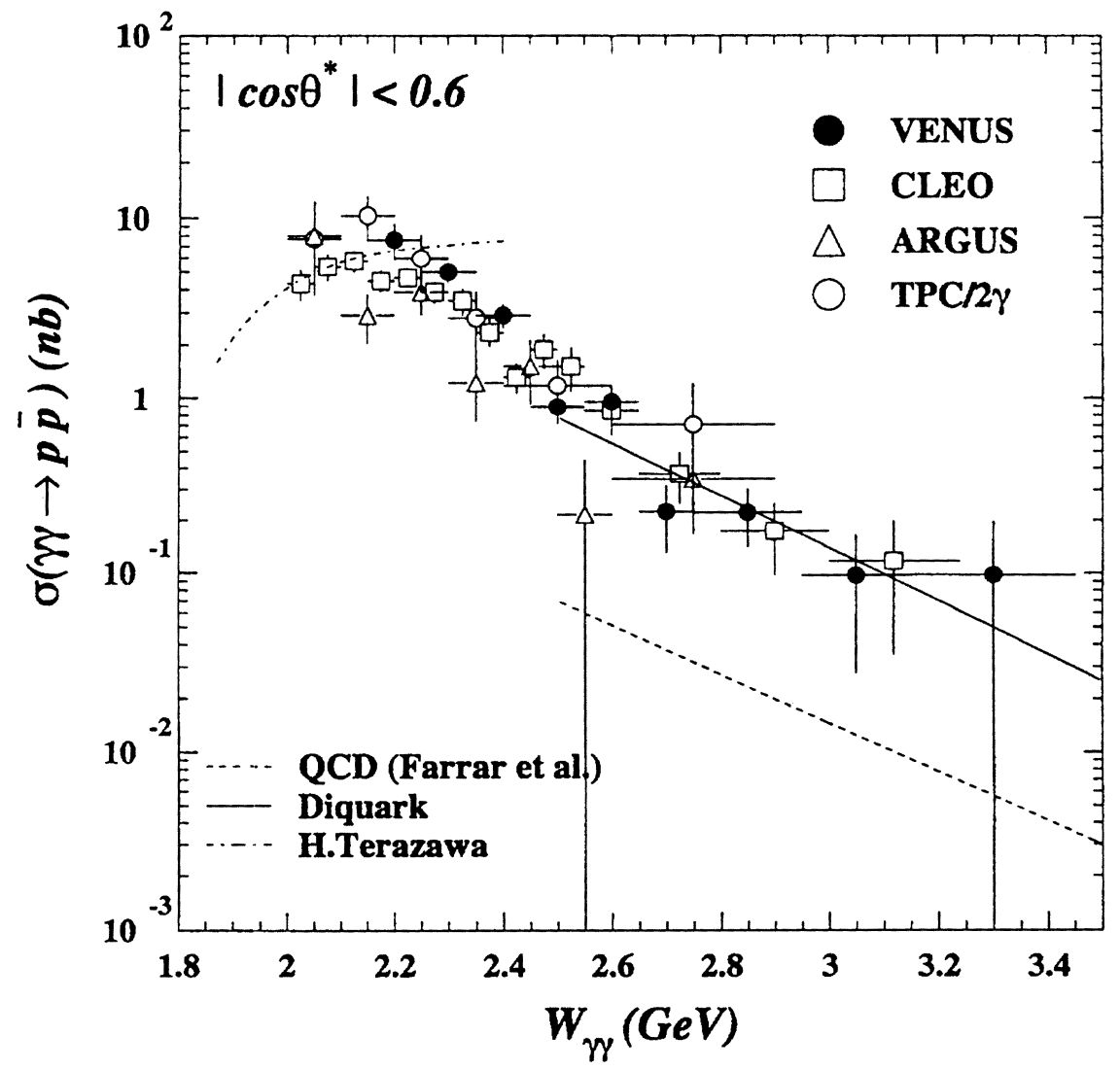

Figure 8.5: Measured cross sections for the reaction $\gamma \gamma \rightarrow \bar{p} p$ for $\left|\cos \theta^{*}\right| \leq 0.6$, as a function of CM energy. [54] 
Kroll states that $\sigma\left(\left|\cos \theta^{*}\right| \leq 0.2\right)=34 \mathrm{pb}$. In the calculations which follow, we will use our slightly lower estimate.

We can estimate a continuum cross section for $\bar{p} p \rightarrow \gamma \gamma$ from the above measurement of $\sigma(\gamma \gamma \rightarrow \bar{p} p)$ by CLEO and VENUS by using the principle of detailed balance. In general, for a two-body reaction $a+b \rightarrow c+d$, the cross section is

$$
\sigma(a b \rightarrow c d) \propto\left(2 s_{c}+1\right)\left(2 s_{d}+1\right) \times p_{c, d}^{2} \times\left|\mathcal{M}_{a b \rightarrow c d}\right|^{2}
$$

where $s_{c}$ and $s_{d}$ are the spins of the final state particles, $p_{c, d}$ the momentum of one of the final state particles in the center of mass frame, and $\mathcal{M}_{a b \rightarrow c d}$ is the matrix element for the process. The principle of detailed balance states that the matrix elements for the two inverse processes are the same, i.e. that $\mathcal{M}_{a b \rightarrow c d}=\mathcal{M}_{c d \rightarrow a b}$. Thus, we can relate the cross sections for the reaction $a+b \rightarrow c+d$ to that for the reaction $c+d \rightarrow a+b$ by the following:

$$
\sigma(a b \rightarrow c d)=\frac{\left(2 s_{c}+1\right)\left(2 s_{d}+1\right)}{\left(2 s_{a}+1\right)\left(2 s_{b}+1\right)} \times \frac{p_{c, d}^{2}}{p_{a, b}^{2}} \times \sigma(c d \rightarrow a b) .
$$

We will now apply the results of Eq. 8.2 to the problem at hand.

At $\sqrt{s}=3.0 \mathrm{GeV}$, the center of mass momentum of the $\bar{p}$ is equal to $p(\bar{p})=$ $\sqrt{s / 4-m^{2}(\bar{p})}=1.17 \mathrm{GeV}$. The center of mass momentum of the $\gamma$ is simply 1.5 $\mathrm{GeV}$, therefore the momentum factor $\left(p_{c, d}^{2} / p_{a, b}^{2}\right)=1.64$.

The products of spin factors $\left(2 s_{1}+1\right)\left(2 s_{2}+1\right)$ require some careful consideration. While it is obvious that the proton and antiproton, being fermions with spin $\mathrm{s}=1 / 2$, each have spin factors $(2 s+1)=2$, the photon spin factor is not $(2 s+1)=3$ as one might think, considering that the photon spin is 1 .

In the reaction $\bar{p} p \rightarrow \gamma \gamma$, two real photons are produced. Real photons can have 


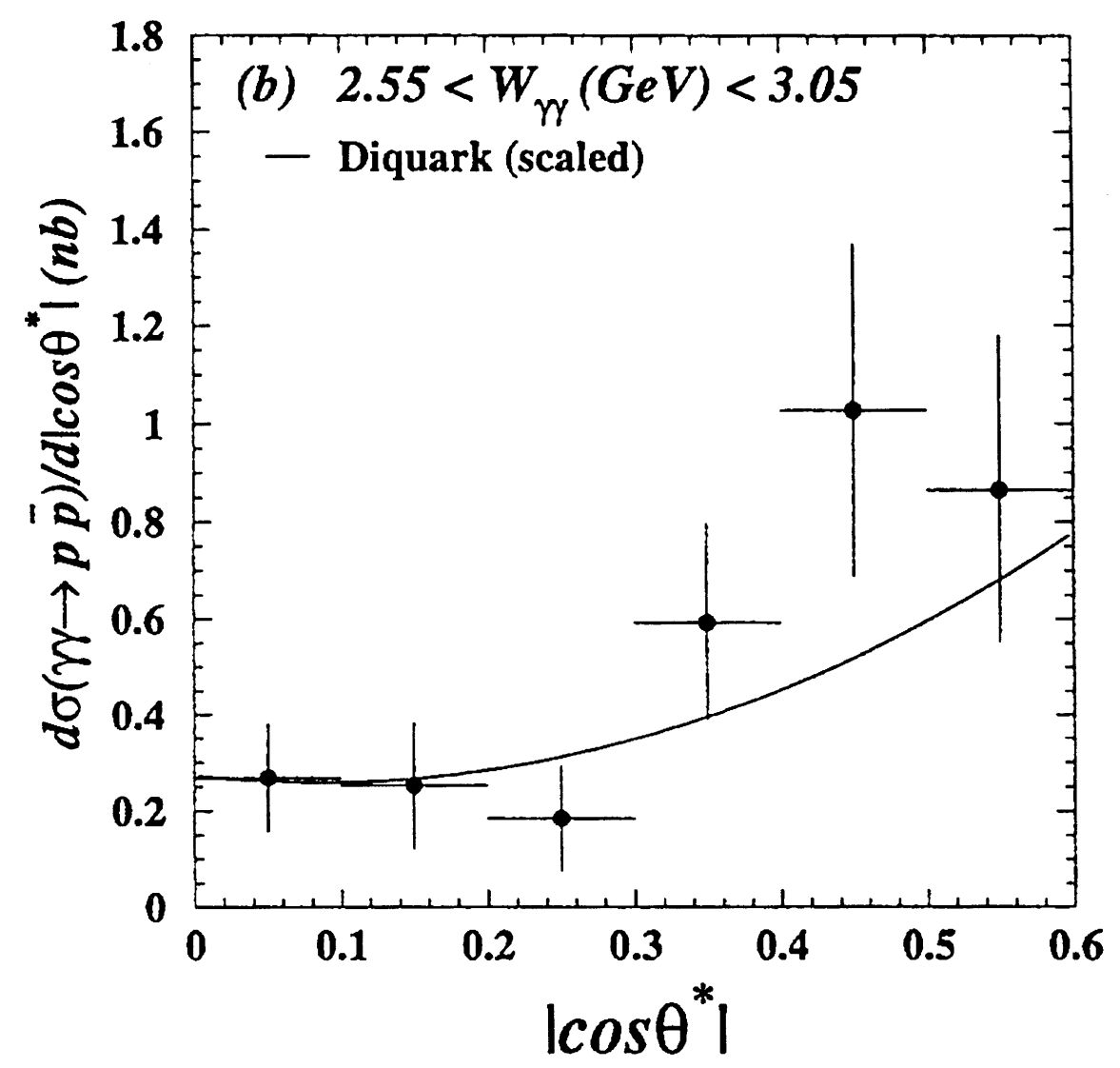

Figure 8.6: Measured differential cross section for the reaction $\gamma \gamma \rightarrow \bar{p} p$ from VENUS, for $\mathrm{CM}$ energies $2.55<W<3.05 \mathrm{GeV}$. [54] 
only helicities +1 or -1 , i.e., the $z$ component of their spins can only be \pm 1 . Consequently, the factors $(2 s+1)$ for the photons must be replaced by 2 , and not 3 .

Therefore, at $\sqrt{s}=3.0 \mathrm{GeV}$,

$$
\sigma(\bar{p} p \rightarrow \gamma \gamma)=1.64 \times \sigma(\gamma \gamma \rightarrow \bar{p} p)
$$

Thus, we estimate the continuum cross section for $\bar{p} p \rightarrow \gamma \gamma$ at $\sqrt{s}=3.0 \mathrm{GeV}$, integrated over $\left|\cos \theta^{*}\right| \leq 0.2$, to be $28 \mathrm{pb} \times 1.64 \simeq 46 \pm \leq 23 \mathrm{pb}$.

This measured continuum cross section contribution of $46 \pm \leq 23 \mathrm{pb}$ at $\sqrt{s}=$ $3000 \mathrm{MeV}$ is large enough for us to question the reliability of the E760 results which do not take this into account. It is indeed instructive to find out how much the E760 results would change if a continuum cross section contribution were added to the background estimate based on the feed-down calculations alone. We have tried to estimate this change by fitting the E760 data with the feed-down background alone, and also with the feed-down background increased (very conservatively) by just $30 \%$ of the CLEO/VENUS contribution, i.e., by $14 \mathrm{pb}$ at the resonance energy, and having the same functional form as the feed-down background. The two fits are shown in Fig. 8.7. It is found that the resonance mass remains unchanged, but the total and partial widths change substantially. The results are:

Feed-down background (FB) only (same as in Ref. [50]):

$$
\Gamma\left(\eta_{c}\right)=23.8_{-7.0}^{+12.8} \mathrm{MeV}, \quad \Gamma\left(\eta_{c} \rightarrow \gamma \gamma\right)=6.6_{-2.4}^{+3.9} \mathrm{keV}
$$

$\mathrm{FB}+0.3 \times$ measured continuum contribution:

$$
\Gamma\left(\eta_{c}\right)=19.0_{-6.5}^{+11.5} \mathrm{MeV}, \quad \Gamma\left(\eta_{c} \rightarrow \gamma \gamma\right)=4.3_{-1.9}^{+2.9} \mathrm{keV}
$$




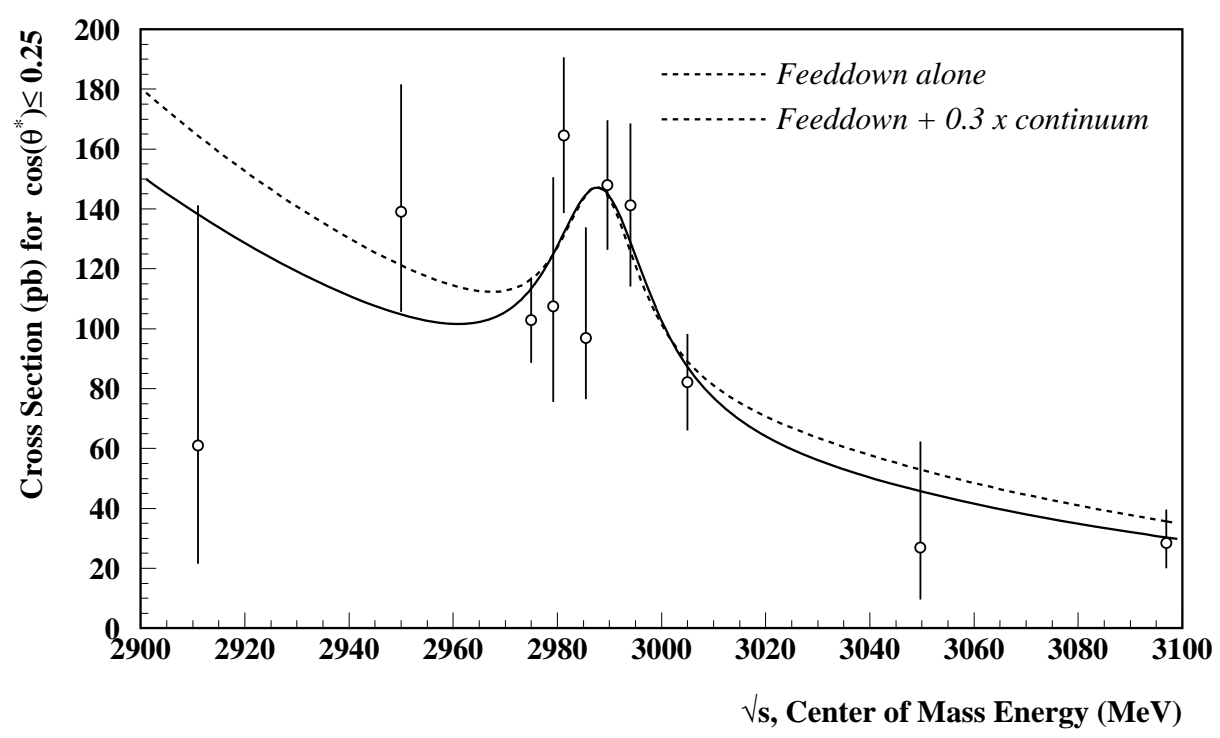

Figure 8.7: Results of two fits to the E760 $\gamma \gamma$ cross sections. The solid line represents the result of a fit to the $\eta_{c}$ Breit-Wigner and the calculated feed-down background alone, while the dashed line represents the result of a fit to the $\eta_{c}$ Breit-Wigner and the feed-down background plus $30 \%$ of the continuum cross sections estimated from the CLEO/VENUS measurements of $\sigma(\gamma \gamma \rightarrow \bar{p} p)$.

This exercise tells us that while we may not have an exact measure of the continuum cross section and/or its variation with $\sqrt{s}$, it is rather dangerous to rely on the calculated feed-down cross sections as a measure of the total background. It is far more prudent to not make any a priori assumptions about the background, and to let the data place it wherever it does in a fit in which all parameters are free. 


\section{Present Approach to the Background Problem}

In view of the above discussion about the perils of using feed-down calculation estimates as measures of the true background, we have investigated several alternative approaches to the background problem.

The first point to settle is about the functional form to be assigned to the background variation with $\sqrt{s}$. E760 claimed to determine the background 'shape' via a fit to the high statistics feed-down cross sections. The functional form

$$
\sigma_{b k g}=A \times\left(\frac{\sqrt{s_{0}}}{\sqrt{s}}\right)^{B}
$$

was found to fit the data rather well in the small, separate energy regions, $2900 \leq$ $\sqrt{s}(\mathrm{MeV}) \leq 3100$ for $\eta_{c}$ and $3524 \leq \sqrt{s}(\mathrm{MeV}) \leq 3686$ for $\eta_{c}^{\prime}$. No attempt was made to find a single functional form for the entire energy region $2900 \leq \sqrt{s}(\mathrm{MeV}) \leq 3686$. Our situation is different in two respects.

First, since we have chosen not to use high statistics cross sections from a feeddown calculation, we have at our disposal only the measured cross sections with their unflattering statistical errors. These errors are reasonably small in the $\chi_{2}, \eta_{c}^{\prime}$ region, $3500 \leq \sqrt{s}(\mathrm{MeV}) \leq 3800 \mathrm{MeV}$, but are quite large in the $\eta_{c}$ region, particularly below the resonance.

Second, since we have data, more or less continuously, in the entire energy region $2900 \leq \sqrt{s}(\mathrm{MeV}) \leq 3800 \mathrm{MeV}$, we feel that a single functional fit should be obtained for this entire region. This is not easy to begin with, but is rendered more difficult because of the great disparity between the statistical precision of the data in the low energy region, $\sqrt{s} \leq 3400 \mathrm{MeV}$, and in the high energy region, $\sqrt{s} \geq 3400 \mathrm{MeV}$. As a result, a $\chi^{2}$ or maximum likelihood fit tends to, in effect, decouple the two regions, 


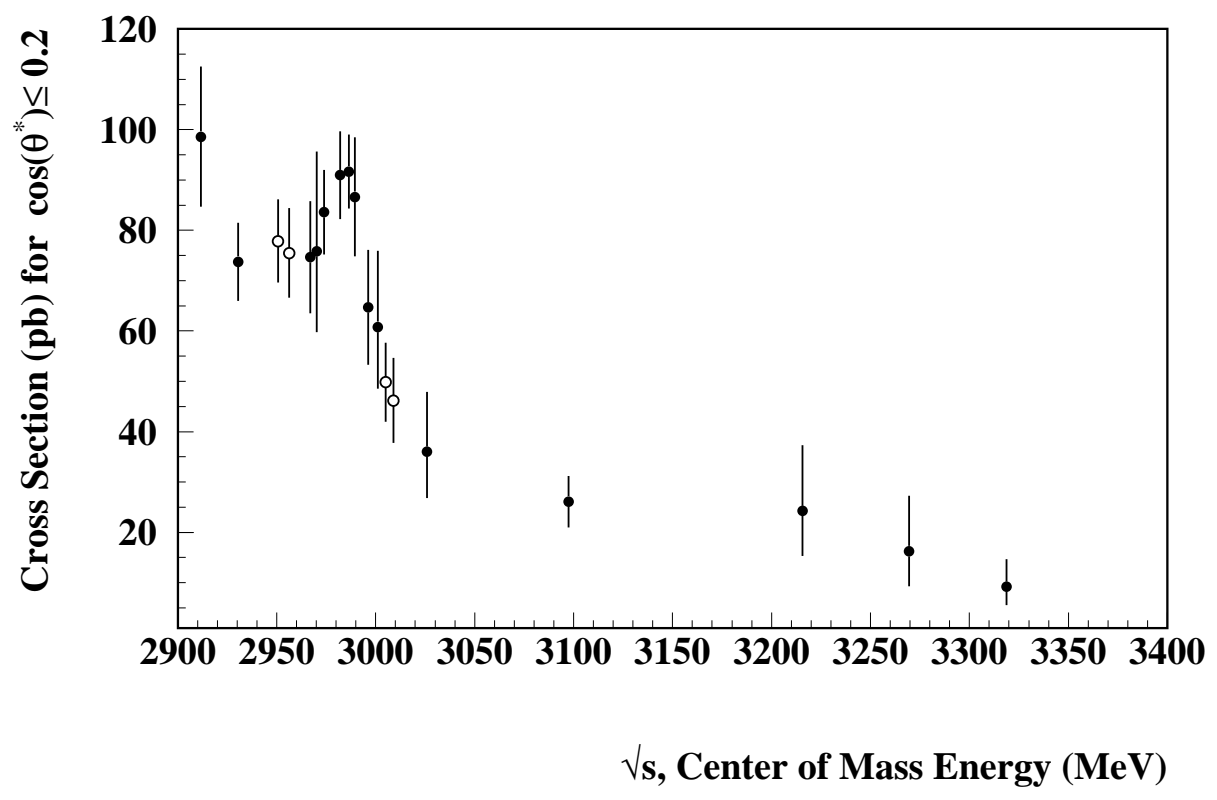

Figure 8.8: $\gamma \gamma$ Cross Sections for $\left|\cos \theta^{*}\right| \leq 0.2$, for CM energies from 2900 to $3400 \mathrm{MeV}$. The data points represented by the open circles are those which we have considered either "background" or "resonance" points in our attempt to determine the form of the background.

with large uncertainty in the parameters which control the low energy region, and much smaller errors in the parameters which control the high energy region.

We have tried several functional forms to fit the variation of the background with $\sqrt{s}$. All such attempts suffer from the problems caused by the lack of statistical precision in the low energy cross sections, and from how we define the 'background' region in the vicinity of the $\eta_{c}$ resonance. We describe the results of our investigations below. 
After much searching, the best functional form we have determined is

$$
A \times\left(\frac{2985}{\sqrt{s}}\right)^{B}+C \times\left(\frac{3556}{\sqrt{s}}\right)^{D} .
$$

As we mentioned above, it is important to specify which of the data points should be considered "resonance" points, and which should be considered "background" points. The choice is crucial, especially if the number of "background" points is small. In Fig. 8.8, which shows the data in the $\eta_{c}$ region, it is clear that the points at $3025 \mathrm{MeV}$ or above, and at $\sim 2930 \mathrm{MeV}$ or below, are far enough away from $\eta_{c}$ to be considered background. The data points at 2950 and $2956 \mathrm{MeV}$, and 3004 and $3009 \mathrm{MeV}$, indicated by the open circles, could conceivably be considered either background or resonance points. This ambiguity gives rise to a large variation of the background determination, depending on which of the points we include in the background fit.

We have studied this variation by fitting the data to Eq. 8.7, always excluding the $\chi_{0}, \chi_{2}$ and $\eta_{c}^{\prime}$ points, and those between 2960 and $3001 \mathrm{MeV}$. In each fit, we also excluded some, or all, of the four points mentioned above. A summary of the results of this study is shown in Table 8.1. The background functions resulting from the different fits are presented in Fig. 8.9. These results show that the parameters $C$ and $D$, which determine the fit in the high energy region, remain stable because of the good statistical precision of the data in that region. However, the parameters $A$ and $B$ vary by large amounts, leading to up to $\sim 10 \mathrm{pb}$ variation in the level of the background at $\sim 2988 \mathrm{MeV}$. This investigation makes it clear that because of our poor statistics we cannot make an independent determination of the background. We have therefore chosen to fit the entireity of our data, including both the resonance 
Table 8.1: Summary of results of fitting the background curve (Eq. 8.7) to the data for $\left|\cos \theta^{*}\right| \leq 0.2$. Each line in the table indicates the results of a fit in which only the data points marked by $(\sqrt{ })$ are included. Other points, indicated by $(-)$, are omitted.

\begin{tabular}{|l|llll|llll|}
\hline & \multicolumn{4}{|c|}{ Energy points } & \multicolumn{4}{c|}{ Background Parameters } \\
\hline Set & 2951 & 2956 & 3004 & 3009 & A & B & C & D \\
\hline 1 & $\sqrt{ }$ & $\sqrt{ }$ & $\sqrt{ }$ & $\sqrt{ }$ & 21.91 & 37.94 & 7.71 & 8.37 \\
2 & - & - & - & - & 5.52 & 88.68 & 7.72 & 9.17 \\
\hline 3 & - & - & $\sqrt{ }$ & $\sqrt{ }$ & 12.02 & 52.44 & 7.71 & 9.23 \\
4 & $\sqrt{ }$ & $\sqrt{ }$ & - & - & 21.84 & 38.07 & 7.72 & 8.38 \\
5 & $\sqrt{ }$ & - & - & $\sqrt{ }$ & 18.02 & 43.35 & 7.73 & 8.86 \\
\hline
\end{tabular}

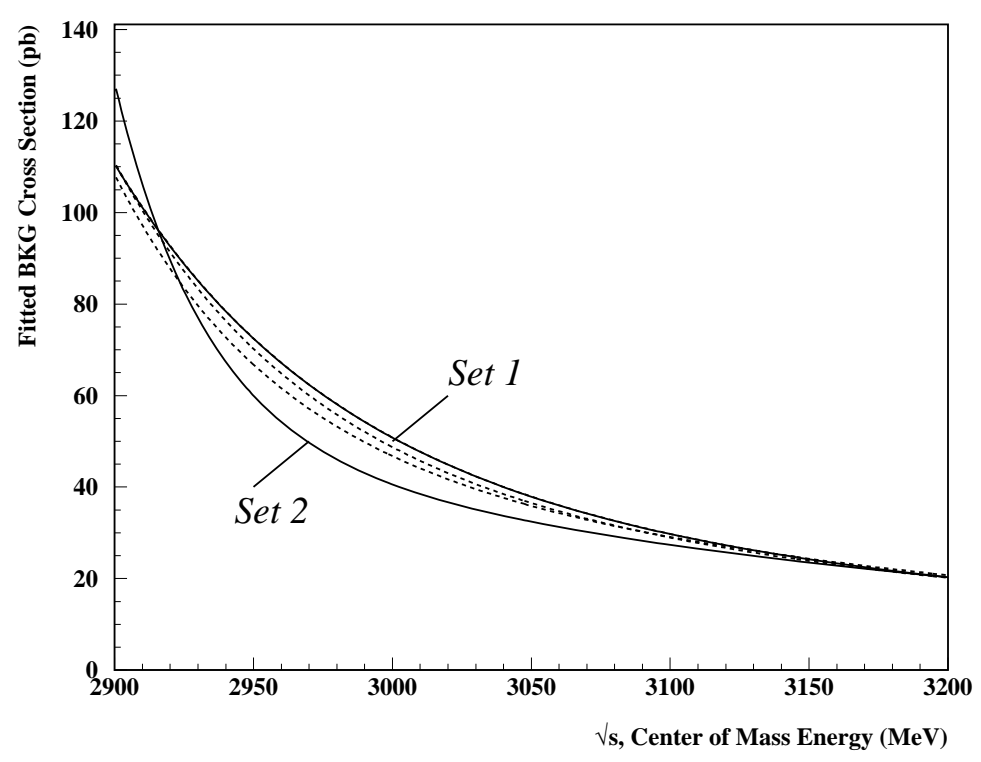

Figure 8.9: Results of fitting the background function (Eq. 8.7) to the data for $\left|\cos \theta^{*}\right| \leq 0.2$. Each fit represents one of the options presented in Table 8.1. Those representing the extreme parameter sets 1 and 2 are indicated explicitly. 
and background points, to the sum of the background cross section of the form of (Eq.8.7) and the Breit-Wigner resonance function, to simultaneously determine the best fit background and resonance parameters.

\subsection{Determination of Resonance and Background Parameters}

The resonance parameters of the charmonium states for which we report measurements have been obtained by use of a Maximum Likelihood fit to the data. The likelihood function,

$$
L=\prod_{i=1, N} \frac{\nu_{i}^{n_{i}} e^{-\nu_{i}}}{n_{i} !}
$$

is the product of $\mathrm{N}$ Poisson functions $(\mathrm{N}=$ the number of data points over which the fit is performed). The term for the $i$ th data point gives the probability that $n_{i}$ events are observed if $\nu_{i}$ are expected. For a resonance $R$, the $\nu_{i}$ are given by

$$
\nu_{i}=\left[\int \mathcal{L}\right]_{i} \times\left(\epsilon_{i} \alpha \int_{0}^{\infty} g_{\text {beam }}\left(E-\sqrt{s}_{i}\right) \sigma_{B W}(E) d E+\sigma_{b k g}\right),
$$

where $\left[\int \mathcal{L}\right]_{i}$ is the integrated luminosity taken at the $i$ th data point, $\epsilon_{i}$ and $\alpha$ are the overall efficiency and acceptance, respectively. $g_{\text {beam }}\left(E-\sqrt{s}_{i}\right)$ is the measured distribution of the CM energy about the mean CM energy $\sqrt{s}_{i}$, and $\sigma_{B W}$ is the Breit-Wigner cross section

$$
\sigma_{B W}(E)=\frac{4 \pi(2 J+1)}{E^{2}-4 m_{p}^{2}} \frac{B(R \rightarrow \bar{p} p) \times B(R \rightarrow \gamma \gamma)}{1+4\left(E-M_{R}\right)^{2} / \Gamma_{R}^{2}} .
$$

The background cross section, $\sigma_{b k g}$ is parameterized as in Eq. 8.7. Thus, the parameters of the fit function are the resonance mass $M_{R}$, its total width $\Gamma_{R}$ and the 
product of $\bar{p} p$ and $\gamma \gamma$ branching fractions $B(R \rightarrow \bar{p} p) \times B(R \rightarrow \gamma \gamma)$ (or, equivalently, $B(R \rightarrow \bar{p} p) \times \Gamma(R \rightarrow \gamma \gamma))$, and the four background parameters $A, B, C$ and $D$.

The fit is performed using the CERNLIB routine MINUIT, [57] which finds the point in parameter space which maximizes the natural log of the likelihood function $(\ln L)$. When a maximum is found, the free parameters are each individually varied about the optimum values, while the others are kept constant. The variation in each parameter which results in a change of $\ln L$ by $1 / 2$ (i.e., $\ln L$ decreases to $\left.\ln L_{\max }-1 / 2\right)$ is reported by MINUIT as the one- $\sigma$ error on that parameter.

\subsection{Resonance Parameters of $\eta_{c}$}

The observed $\bar{p} p \rightarrow \gamma \gamma$ cross sections for $\left|\cos \left(\theta^{*}\right)\right| \leq 0.20$ for the scan across the $\eta_{c}$ mass region are shown in Fig. 8.10 as a function of the center of mass energy, $\sqrt{s}$. The data have been fitted, as described above, with all three Breit-Wigner parameters, and all four background parameters allowed to vary in the fit. The solid curve which is superimposed on the data in Fig. 8.10 is the result of this fit.

Our results for the $\eta_{c}$ resonance parameters are:

$$
\begin{gathered}
M\left(\eta_{c}\right)=2982.4_{-2.2}^{+2.3} \mathrm{MeV} \\
\Gamma\left(\eta_{c}\right)=26.9_{-9.5}^{+10.8} \mathrm{MeV} \\
B\left(\eta_{c} \rightarrow \bar{p} p\right) \times B\left(\eta_{c} \rightarrow \gamma \gamma\right)=25.3_{-4.5}^{+4.6} \times 10^{-8} .
\end{gathered}
$$

or, equivalently,

$$
B\left(\eta_{c} \rightarrow \bar{p} p\right) \times \Gamma\left(\eta_{c} \rightarrow \gamma \gamma\right)=6.8_{-2.8}^{+3.5} \mathrm{eV}
$$



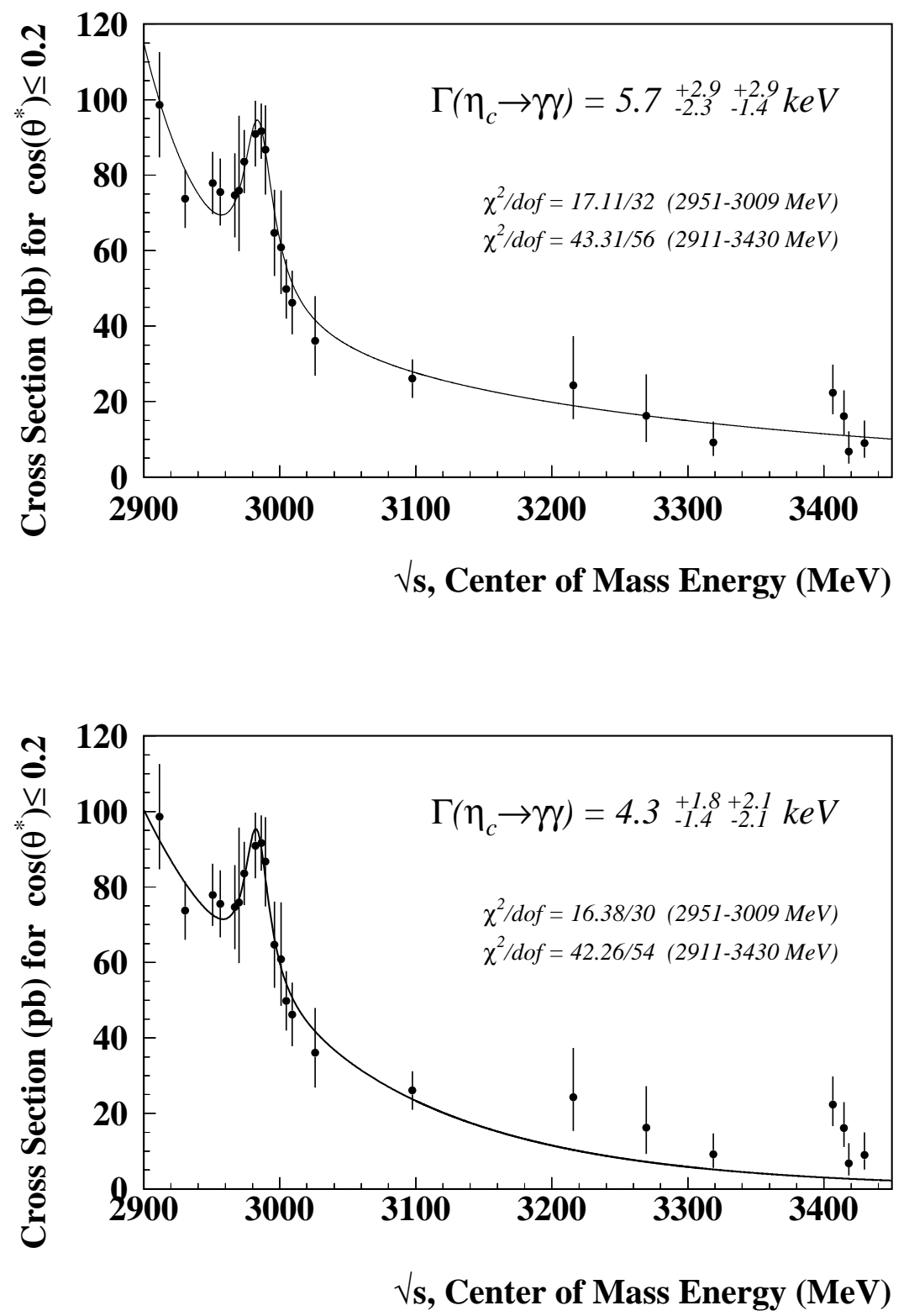

Figure 8.10: Measured cross sections for $\bar{p} p \rightarrow \gamma \gamma$ for $\left|\cos \theta^{*}\right| \leq 0.2$ in the $\eta_{c}$ region. (top) Our present best fit, to all data points from $\sqrt{s}=2911$ to $3859 \mathrm{MeV}$, with all resonance and background parameters free. (bottom) The result of the fit to the data points in the limited region from $\sqrt{s}=2911 \mathrm{MeV}$ to $3100 \mathrm{MeV}$, using the E760 form of the background parameterization. The values of $\chi^{2} /$ d.o.f for each fit are indicated, calculated for all data points shown, and for the peak (2951-3009 MeV) region. 
Table 8.2: Values of $\eta_{c}$ resonance parameters from PDG94, [58] E760 [50] and E835. It should be noted that the E760 results are those which were determined by essentially fixing the background to the values determined by the feeddown calculation, a procedure which results in substantially lower errors.

\begin{tabular}{|l|l|ll|l|}
\hline Parameter & PDG94 & $\begin{array}{l}\text { E760 } \\
(\leq 0.25)\end{array}$ & $\begin{array}{l}\text { E760 } \\
(\leq 0.20)\end{array}$ & $\begin{array}{l}\text { E835 } \\
(\leq 0.20)\end{array}$ \\
\hline$M\left(\eta_{c}\right)(\mathrm{MeV})$ & $2979.8 \pm 1.9$ & $2988.3_{-3.1}^{+3.3}$ & $2988.8_{-2.2}^{+2.4}$ & $2982.4_{-2.2}^{+2.3}$ \\
$\Gamma\left(\eta_{c}\right)(\mathrm{MeV})$ & $10.3_{-3.4}^{+3.8}$ & $23.9_{-7.1}^{+12.6}$ & $19.0_{-5.2}^{+8.0}$ & $26.9_{-9.5}^{+10.8}$ \\
$B_{\bar{p} p} \times B_{\gamma \gamma} \times 10^{8}$ & $68_{-31}^{+42}$ & $33.6_{-7.0}^{+8.0}$ & $44.9_{-8.8}^{+10.2}$ & $25.3_{-4.5}^{+4.6}$ \\
$B_{\bar{p} p} \times \Gamma\left(\eta_{c} \rightarrow \gamma \gamma\right)(\mathrm{eV})$ & & $8.1_{-2.0}^{+2.9}$ & $8.6_{-1.9}^{+2.5}$ & $6.8_{-2.8}^{+3.5}$ \\
\hline$B_{\bar{p} p} \times 10^{4}$ & $12 \pm 4$ & & & \\
\hline$B\left(\eta_{c} \rightarrow \gamma \gamma\right) \times 10^{4}$ & $6_{-5}^{+6}$ & $2.8_{-0.6-0.7}^{+0.7+1.4}$ & $3.0 \pm 0.8_{-0.8}^{+1.4}$ & $2.1 \pm 0.4_{-0.5}^{+1.1}$ \\
$\Gamma\left(\eta_{c} \rightarrow \gamma \gamma\right)(\mathrm{keV})$ & $7.2_{-1.7}^{+2.0}$ & $6.7_{-1.7-1.7}^{+2.4+3.4}$ & $7.2_{-1.6-1.8}^{+2.1+3.6}$ & $5.7_{-2.3-1.4}^{+2.9+2.9}$ \\
\hline Signal events & \multicolumn{3}{|l|}{77} & 260 \\
\hline
\end{tabular}

$\dagger$ Events in $2911 \leq \sqrt{s}(\mathrm{MeV}) \leq 3100$ region

We note that the errors on Eq. 8.12 are not obtained by dividing Eq. 8.11 by $\Gamma\left(\eta_{c}\right)$. They are obtained by a separate fit.

The best fit values for the background parameters for this fit are:

$$
\begin{gathered}
A=12.5_{-7.73}^{+11.41} \mathrm{pb} \quad B=59.0_{-22.5}^{+39.2} \\
C=7.68_{-0.41}^{+0.43} \mathrm{pb} \quad D=8.80_{-1.58}^{+1.27}
\end{gathered}
$$

The errors quoted for the resonance parameters of $\eta_{c}$ have been obtained by Monte Carlo evaluation, as described in Appendix D. The errors obtained by the Maximum Likelihood fit are only slightly different: $M\left(\eta_{c}\right)=2982.4_{-2.4}^{+2.2} \mathrm{MeV}$, $\Gamma\left(\eta_{c}\right)=26.9_{-8.5}^{+12.1} \mathrm{MeV}, B\left(\eta_{c} \rightarrow \bar{p} p\right) \times B\left(\eta_{c} \rightarrow \gamma \gamma\right)=25.3_{-4.5}^{+4.6} \times 10^{-8}, B\left(\eta_{c} \rightarrow \bar{p} p\right) \times$ 
$\Gamma\left(\eta_{c} \rightarrow \gamma \gamma\right)=6.2_{-2.4}^{+4.0} \mathrm{eV}$. For our final results, we use the errors determined by Monte Carlo evaluation.

The resonance parameters are given in Table 8.2, together with the PDG94 averages [58] (which did not include E760 results) and E760 results for both $\left|\cos \theta^{*}\right| \leq$ 0.25 and $\left|\cos \theta^{*}\right| \leq 0.2$. [50]

We recall that the E760 results were obtained by using background parameters which were essentially fixed to the values obtained by using the feed-down calculation. This resulted in errors on $\Gamma\left(\eta_{c}\right), B\left(\eta_{c} \rightarrow \bar{p} p\right) \times B\left(\eta_{c} \rightarrow \gamma \gamma\right)$, and $B\left(\eta_{c} \rightarrow \bar{p} p\right) \times$ $\Gamma\left(\eta_{c} \rightarrow \gamma \gamma\right)$ which were $\sim 30 \%$ to $50 \%$ smaller than those obtained without this 'fixing'. This should be kept in mind when comparing the present results with those of E760.

In order to carry the comparison with E760 one step closer, we have also fitted our data in the limited region near $\eta_{c}$, from 2911 to $3100 \mathrm{MeV}$, using the functional form for the background used in E760, $\sigma_{b k g}=A \times\left(\sqrt{s_{0}} / \sqrt{s}\right)^{B}$. The results for the $\eta_{c}$ resonance and background parameters using this method are:

$$
\begin{aligned}
& M\left(\eta_{c}\right)=2982.99_{-2.14}^{+2.23} \mathrm{MeV} \\
& \Gamma\left(\eta_{c}\right)=22.4_{-6.9}^{+8.4} \mathrm{MeV} \\
& B\left(\eta_{c} \rightarrow \bar{p} p\right) \times B\left(\eta_{c} \rightarrow \gamma \gamma\right)=22.9_{-3.9}^{+4.1} \times 10^{-8} \\
& B\left(\eta_{c} \rightarrow \bar{p} p\right) \times \Gamma\left(\eta_{c} \rightarrow \gamma \gamma\right)=5.1_{-1.7}^{+2.2} \mathrm{eV} \\
& B\left(\eta_{c} \rightarrow \gamma \gamma\right)=1.9 \pm 0.3_{-0.5}^{+1.0} \times 10^{-4} \\
& \Gamma\left(\eta_{c} \rightarrow \gamma \gamma\right)=4.3_{-1.4-2.1}^{+1.8+2.1} \mathrm{keV} \\
& A=53.6_{-4.5}^{+4.2} \mathrm{pb} B=22.0_{-3.3}^{+3.5}
\end{aligned}
$$

and the result of the fit is shown in Fig. 8.10. (Note that the background parameters obtained by E760 were very similar: $\left.A_{b k g}=49.0 \pm 1.3 \mathrm{pb}, B_{b k g}=23.1 \pm 1.8\right)$ The 
poor quality of this fit to the measured background in the region beyond $3200 \mathrm{MeV}$ is quite clear. As stated earlier we consider it more correct to include our knowledge of the background over the entire region $2900 \leq \sqrt{s}(\mathrm{MeV}) \leq 3800$ in the fitting procedure, and we therefore do not include the above results in Table. 8.2.

The stability of the each of the measured quantities with respect to the choice of $\cos \left(\theta^{*}\right)$ cut was checked by varying the value of the cut from 0.12 to 0.40 in eight steps and performing a fit for each $\left|\cos \theta^{*}\right|$ cut value. As is shown in Fig. 8.11, the results for each resonance parameter are quite stable with respect to the choice of cut value.

\subsubsection{The $\eta_{c}$ Mass}

Our result for the $\eta_{c}$ mass, $2982.4_{-2.4}^{+2.2} \mathrm{MeV}$, is $\sim 3.5 \mathrm{MeV}$ larger than the PDG94 average, and $\sim 5.5 \mathrm{MeV}$ smaller than the value reported by E760. Unfortunately, the excellent precision in beam energy determination does not help in this measurement, and the errors on our measurement arise primarily from the fit. The fact that our result for the mass, $M\left(\eta_{c}\right)=2982.4_{-2.2}^{+2.3} \mathrm{MeV}$ differs by two $\sigma$ from the E760 final result of $M\left(\eta_{c}\right)=2988.3_{-3.1}^{+3.3}$ is somewhat disturbing, though perhaps not unexpected because of the limited statistical precision of both data sets.

The errors on our mass measurement are comparable to those for the most precise results of other experiments. Our result is shown together with those from other experiments in Fig. 8.12 and Table 8.3.

Our result agrees with that of the Crystal Ball experiment [63] within $1 \sigma$. 

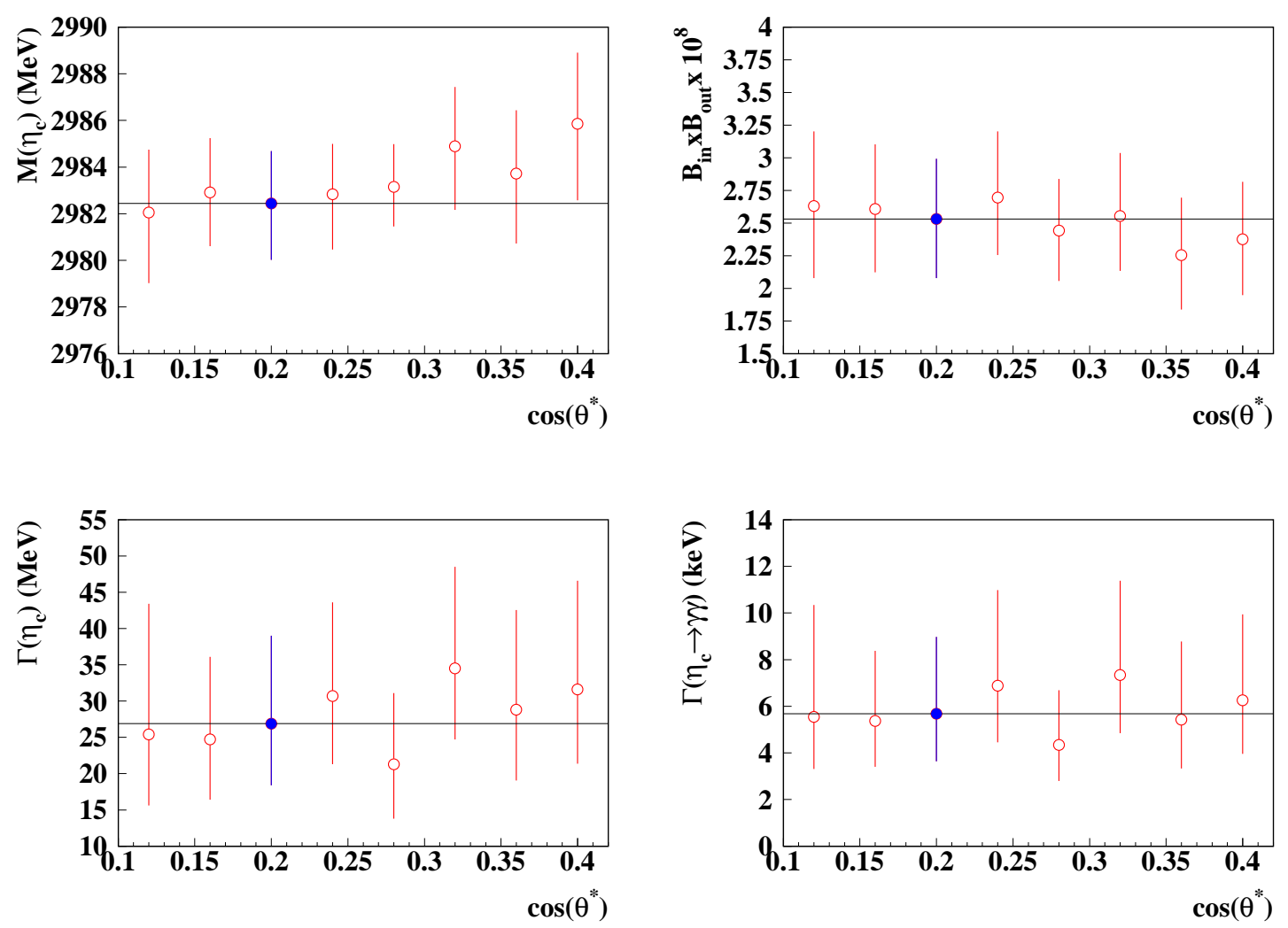

Figure 8.11: Results of a study of the stability of the results for each of the $\eta_{c}$ resonance parameters due to the choice of $\cos \left(\theta^{*}\right)$ cut value. The solid circle and the horizontal line in each plot indicate our final results. 


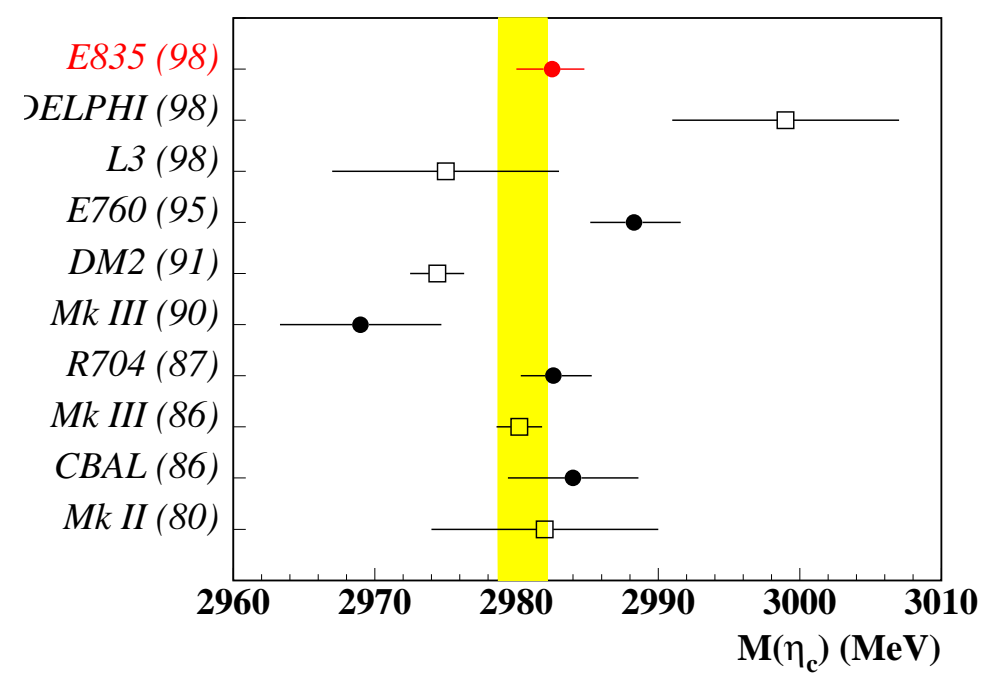

Figure 8.12: Results of measurements of the $\eta_{c}$ mass, for comparison to that obtained in E835. The shaded band indicates the weighted average of the displayed results The results indicated by the open squares are from experiments which observed $\eta_{c}$ in several hadronic decay channels, and presented the average reconstructed mass as their result. The solid circles indicate results from experiments which measure the $\eta_{c}$ mass via a single decay channel.

Table 8.3: Experimental results for the mass of $\eta_{c}$. The weighted averages presented have been calculated according to the method prescribed in PDG98. [2]

\begin{tabular}{lllll}
\hline Result (MeV) & Experiment (year) & Events & Measurement & Reference \\
\hline $2982.4_{-2.2}^{+2.3}$ & E835 (99) & 260 & $\bar{p} p \rightarrow \gamma \gamma$ & \\
$2988.3_{-3.1}^{+3.3}$ & E760 (95) & 76 & $\bar{p} p \rightarrow \gamma \gamma$ & {$[50]$} \\
$2974.4 \pm 1.9$ & DM2 (91) & 439 & $J / \psi \rightarrow \eta_{c} \gamma^{*}$ & {$[59]$} \\
$2969 \pm 4 \pm 4$ & MkIII (90) & 80 & $J / \psi \rightarrow \gamma \mathrm{K}^{+} \mathrm{K}^{-} \mathrm{K}^{+} \mathrm{K}^{-}$ & {$[60]$} \\
$2982.6_{-2.3}^{+2.7}$ & R704 (87) & 12 & $\bar{p} p \rightarrow \gamma \gamma$ & {$[61]$} \\
$2980.2 \pm 1.6$ & MkIII (86) & 430 & $J / \psi \rightarrow \eta_{c} \gamma^{*}$ & {$[62]$} \\
$2984 \pm 2.3 \pm 4.0$ & CBAL (86) & & $J / \psi, \psi^{\prime} \rightarrow \gamma X$ & {$[63]$} \\
$2982 \pm 8$ & MkII (80) & 18 & $\psi^{\prime} \rightarrow \eta_{c} \gamma^{*}$ & {$[64]$} \\
\hline $2980.2 \pm 1.7$ & \multicolumn{4}{c}{ Weighted Average } \\
\hline
\end{tabular}

* Average of several decay modes 
The world average is pulled down, however, largely by the DM2 measurement of $2974.4 \pm 1.9$, and by the Mark III result of $2980.2 \pm 1.6$. Each of these experiments observed $\eta_{c}$ candidates in several hadronic decay modes, and report an average of the reconstructed invariant masses derived from each channel. The statistics in the individual channels range typically from 10 to 50 counts, with a channel or two having over 100 candidates.

Our measurement, and that of E760 and R704, is derived from a scan of the directly-produced resonance, as described in Chapter 4. We observe just one final state, $\gamma \gamma$, and do not reconstruct any invariant mass. Therefore, we do not depend on the knowledge of reconstruction efficiencies and the acceptances for multiple hadronic final states, as do experiments like DM2 and Mark III. Errors in the determination of the momenta and energies of final state particles, and uncertainties in particle identification can affect the results obtained by such a method. This may explain the apparent disagreement between results from this type of experiment and experiments like ours in which $\eta_{c}$ is observed in a single decay mode.

One of the important observables of the charmonium system is the hyperfine splitting. The $1 S$ hyperfine splitting of charmonium system is the mass difference between $J / \psi\left(1^{3} S_{1}\right)$ and $\eta_{c}\left(1^{1} S_{0}\right)$. We find it to be

$$
\Delta M_{1 S}=3096.88 \pm 0.04 \mathrm{MeV}-2982.4_{-2.2}^{+2.3} \mathrm{MeV}=114.5_{-2.2}^{+2.3} \mathrm{MeV}
$$

Predictions of the mass of $\eta_{c}^{\prime}$ (or rather the $2 \mathrm{~S}$ splitting $\Delta m=m\left(\psi^{\prime}\right)-m\left(\eta_{c}^{\prime}\right)$ ) are based upon the $J / \psi-\eta_{c}$ mass difference. We discuss this later in Sec. 8.6. 


\subsubsection{The Total Width of $\eta_{c}$}

Our result for the $\eta_{c}$ total width, $\Gamma\left(\eta_{c}\right)=26.9_{-9.5}^{+10.9} \mathrm{MeV}$, is significantly larger than that from other experiments, with the exception of the E760 result of $23.9_{-7.1}^{+12.6}$. Though we expended $\sim 20 p b^{-1}$ in the $\eta_{c}$ region (i.e. from $2900 \mathrm{MeV}$ to $3100 \mathrm{MeV}$ ), reflecting a 6-fold increase in luminosity over E760, the errors in our width measurement have not improved significantly from those on the E760 result. This is largely due to the poor statistics for the background points. Our result, together with width results from other experiments, is presented in Table 8.4 and in Fig. 8.13 to allow comparison with other experimental results. It is apparent that none of the reported measurements of the total width are particularly precise. Other than our own E760/E835 measurements, the only measurement which has $\leq \pm 50 \%$ error is that from the Crystal Ball experiment. [63]. The difference between the results of $\eta_{c}$ width by E760/E835, which range from 20 to $30 \mathrm{MeV}$, and the Crystal Ball experiment, $\Gamma\left(\eta_{c}\right)=11.5 \pm 4.5$, is large, and warrants some discussion.

The Crystal Ball result depends not only on the statistics, but on the knowledge of the energy resolution of the photon detector, while ours does not. Therefore any error in the determination of the energy resolution may have a bearing on the Crystal Ball width measurement. The inclusive photon spectrum obtained by Crystal Ball is presented in Fig. 8.14. The three panels of the figure represent three different selections of data.

The Crystal Ball claims an intrinsic photon resolution (FWHM) of $\Gamma=12 \pm 1$ $\mathrm{MeV}$ for the $\sim 108 \mathrm{MeV}$ photons from $J / \psi \rightarrow \gamma \eta_{c}$. Their reported total width of 


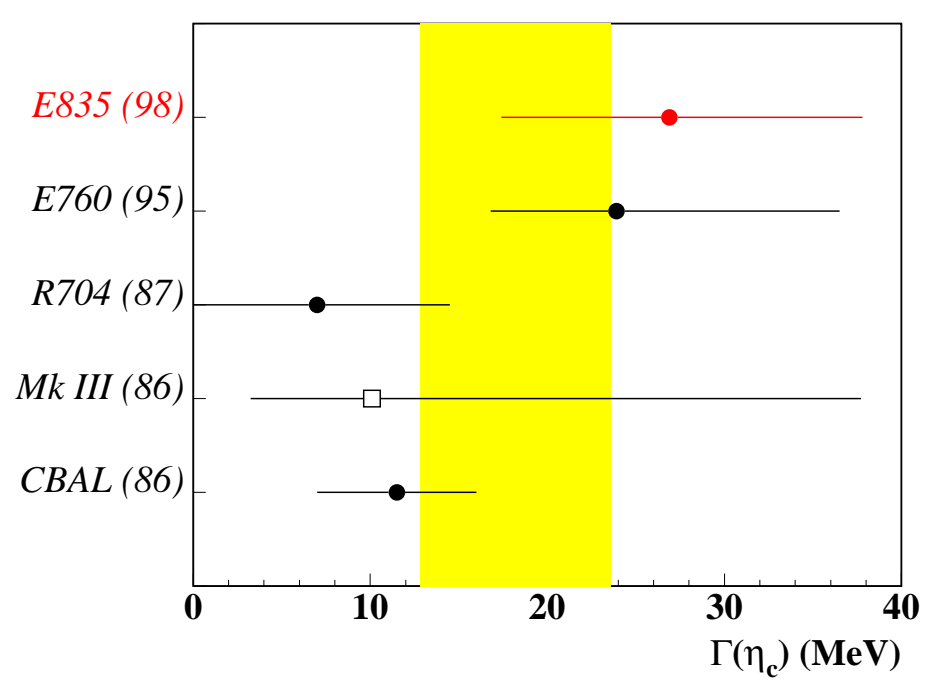

Figure 8.13: Experimental measurements of the $\eta_{c}$ total width, for comparison to the E835 result. The shaded band indicates the weighted average of the displayed results. The Mark III result, indicated with the open square, is shown with errors corresponding to a $90 \%$ confidence interval.

Table 8.4: Experimental results for $\Gamma\left(\eta_{c}\right)$. The weighted averages presented have been calculated according to the method prescribed in PDG98. [2]

\begin{tabular}{lllll}
\hline Result (MeV) & Experiment (year) & Events & Measurement & Reference \\
\hline $26.9_{-9.5}^{+10.8}$ & E835 (99) & 260 & $\bar{p} p \rightarrow \gamma \gamma$ & \\
$24.3_{-7.6}^{+12.6}$ & E760 (95) & 76 & $\bar{p} p \rightarrow \gamma \gamma$ & {$[50]$} \\
$7.0_{-7.0}^{+7.5}$ & R704 (87) & 12 & $\bar{p} p \rightarrow \gamma \gamma$ & {$[61]$} \\
$10.1_{-8.2}^{+33.0}$ & MkIII (86) & 23 & $J / \psi \rightarrow \gamma \bar{p} p^{*}$ & {$[62]$} \\
$11.5 \pm 4.5$ & CBAL (86) & & $J / \psi, \psi^{\prime} \rightarrow \gamma X$ & {$[63]$} \\
\hline $18.2 \pm 5.3$ & Weighted Average & & & \\
\hline
\end{tabular}

* Errors correspond to $90 \%$ confidence interval. 


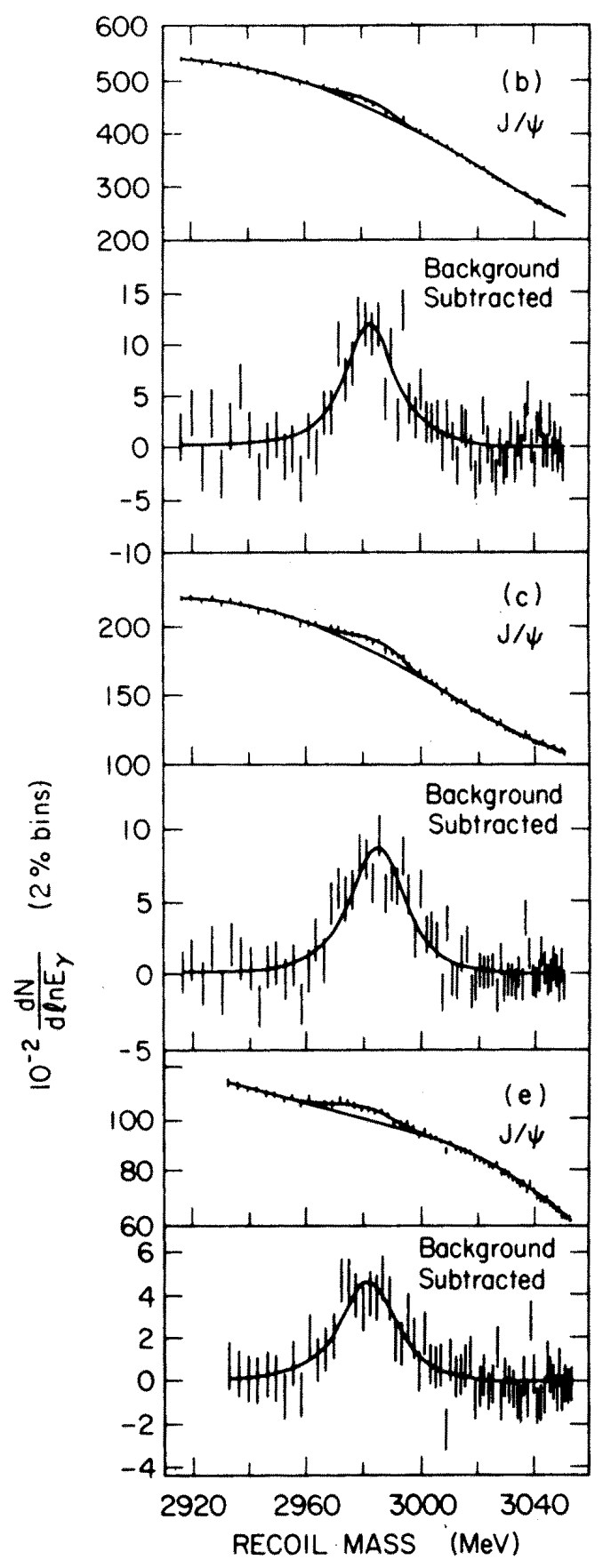

Figure 8.14: Inclusive photon spectra from $J / \psi \rightarrow \gamma X$ obtained by the Crystal Ball. The three sets of panels, indicated by (b), (c) and (e), represent three different selections of their data. 
$\eta_{c}, \Gamma\left(\eta_{c}\right)=11.5 \pm 4.5 \mathrm{MeV}$, added in quadrature would lead to an observed width of $\sim 17 \mathrm{MeV}$. However, the fits presented in Fig. 8.14 appear to have FWHM of $\sim 20-22 \mathrm{MeV}$, which corresponds to a $\sim 50 \%$ larger width than quoted. On the other hand, we cannot rely on the widths estimated from a figure in order to critique the Crystal Ball measurement.

It is apparent from Fig. 8.14 that the Crystal Ball measurements have a signal to background ratio of $\sim 1: 20$. Despite large statistics, background subtraction in such cases can be problematic and non-unique. This could also contribute to uncertainty in the width measurement by the Crystal Ball.

To be fair, we must point out once again that our own results for $\Gamma\left(\eta_{c}\right)$ depends rather sensitively on how we parameterize and fit the background. The width was found to vary between $\sim 20$ and $\sim 33 \mathrm{MeV}$ depending on which background set of Table 8.1 was used. We conclude that an improved direct measurement of the all-important width of $\eta_{c}$ is possible with the $\bar{p} p$ annihilation technique, but only if hardware improvements to substantially reduce the feed-down background are made, and background is measured with high statistical precision over a wide range of energies about $\eta_{c}$.

\subsubsection{The Two Photon Partial Width of $\eta_{c}$}

We have measured the product $B\left(\eta_{c} \rightarrow \bar{p} p\right) \times \Gamma\left(\eta_{c} \rightarrow \gamma \gamma\right)$ to be

$$
B\left(\eta_{c} \rightarrow \bar{p} p\right) \times \Gamma\left(\eta_{c} \rightarrow \gamma \gamma\right)=6.8_{-2.8}^{+3.5} \mathrm{eV}
$$


We obain the two photon partial width of $\eta_{c}$ by dividing this by the PDG value for the branching fraction $B\left(\eta_{c} \rightarrow \bar{p} p\right)=(1.2 \pm 0.4) \times 10^{-3}$. [2] Thus,

$$
\Gamma\left(\eta_{c} \rightarrow \gamma \gamma\right)=5.7_{-2.3-1.4}^{+2.9+2.9} \mathrm{keV}
$$

The first error on $\Gamma\left(\eta_{c} \rightarrow \gamma \gamma\right)$ is statistical, while the second is the asymmetric error which arises from the $33 \%$ error on the branching fraction $B\left(\eta_{c} \rightarrow \bar{p} p\right)$. (See Appendix D) The result of the present measurement is shown for comparison with results from other measurements in Fig. 8.15 and Table 8.5. All measurements of $\Gamma\left(\eta_{c} \rightarrow \gamma \gamma\right)$, other than those of E835, E760 and R704, come from photon-photon fusion experiments. A short description of these experiments is therefore in order.

\section{$\gamma \gamma$ Fusion Experiments}

Experiments like CLEO and the LEP experiments can produce C-even charmonium states, like $\eta_{c}\left(J^{P C}=0^{-+}\right)$and $\chi_{2}\left(J^{P C}=2^{++}\right)$, in $\gamma \gamma$ fusion, i.e., in the reaction

$$
e^{+} e^{-} \rightarrow e^{+} e^{-} \gamma \gamma, \gamma \gamma \rightarrow(c \bar{c}) \rightarrow \text { hadrons }
$$

Such experiments must contend with low effective luminosity for the $\gamma \gamma$ interaction. Two examples of results on charmonium states from $\gamma \gamma$ fusion at CLEO are shown in Fig. 8.16.

$\gamma \gamma$ fusion experiments offer a logical choice for the measurement of $\gamma \gamma$ partial widths, for they measure $\Gamma(R \rightarrow \gamma \gamma)$, rather than $B(R \rightarrow \gamma \gamma)$. However, the event statistics in these experiments are usually poor and spread over several decay channels whose branching ratios are also poorly known. For example, for $\eta_{c}$, no branching 


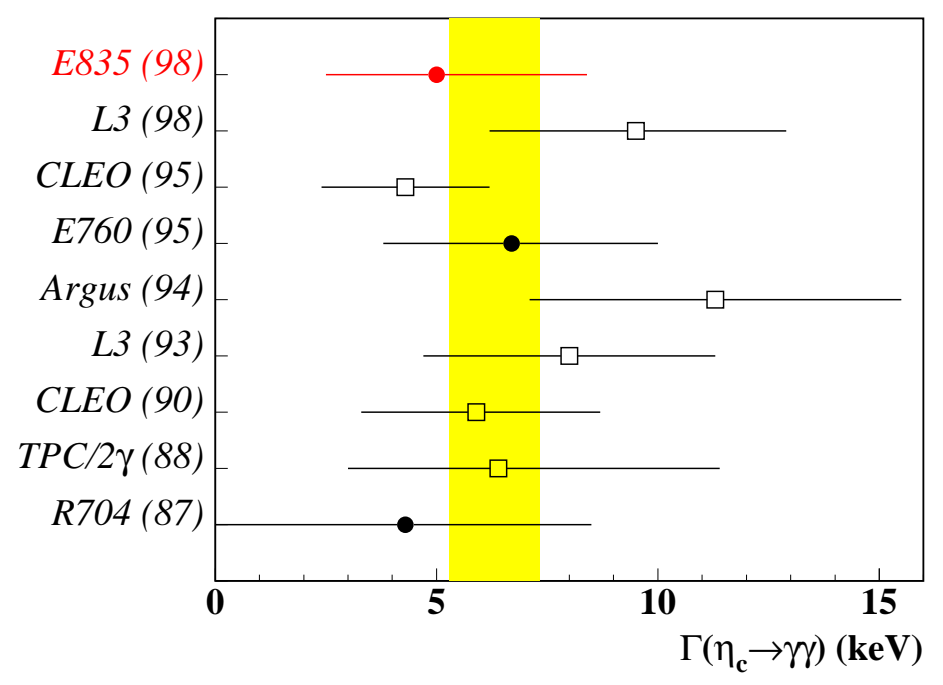

Figure 8.15: Experimental measurements of $\Gamma\left(\eta_{c} \rightarrow \gamma \gamma\right)$, for comparison to the E835 result. The shaded band indicates the weighted average of the displayed results. The open squares indicate results from $\gamma \gamma$ fusion experiments. The solid circles indicate results from $\bar{p} p$ annihilation experiments.

Table 8.5: Experimental results for $\Gamma\left(\eta_{c} \rightarrow \gamma \gamma\right)$. The weighted average presented has been calculated according to the method prescribed in PDG98. [2]

\begin{tabular}{|c|c|c|c|c|}
\hline Result (keV) & Experiment (year) & Events & Measurement & Reference \\
\hline $5.7_{-2.3-1.4}^{+2.9+2.9}$ & $\mathrm{E} 835(99)$ & 260 & $\bar{p} p \rightarrow \gamma \gamma$ & \\
\hline $9.5_{-1.9}^{+2.1} \pm 2.7$ & $\mathrm{~L} 3(98)$ & 108 & $\gamma \gamma \rightarrow$ hadrons ${ }^{*}$ & [65] \\
\hline $4.3 \pm 1.0 \pm 1.6$ & CLEO $(95)$ & 54 & $\gamma \gamma \rightarrow \pi^{+} \pi^{-} K^{ \pm} \pi^{\mp}$ & [66] \\
\hline $6.7_{-1.7-1.7}^{+2.4+3.4}$ & E760(95) & 76 & $\bar{p} p \rightarrow \gamma \gamma$ & {$[50]$} \\
\hline $11.3 \pm 4.2$ & $\operatorname{Argus}(94)$ & 57 & $\gamma \gamma \rightarrow$ hadrons ${ }^{*}$ & [67] \\
\hline $8.0 \pm 2.3 \pm 2.4$ & L3(93) & 17 & $\gamma \gamma \rightarrow$ hadrons ${ }^{*}$ & [68] \\
\hline $5.9_{-1.8}^{+2.1} \pm 1.9$ & CLEO $(90)$ & 15 & $\gamma \gamma \rightarrow$ hadrons* & [69] \\
\hline $6.4_{-3.4}^{+5.0}$ & $\mathrm{TPC} / 2 \gamma(88)$ & 4 & $\gamma \gamma \rightarrow$ hadrons ${ }^{*}$ & {$[70]$} \\
\hline $4.3_{-3.7-1.1}^{+3.4}$ & $\mathrm{R} 704(86)$ & 12 & $\bar{p} p \rightarrow \gamma \gamma$ & [61] \\
\hline $6.3 \pm 1.0$ & Weighted Average & & & \\
\hline
\end{tabular}



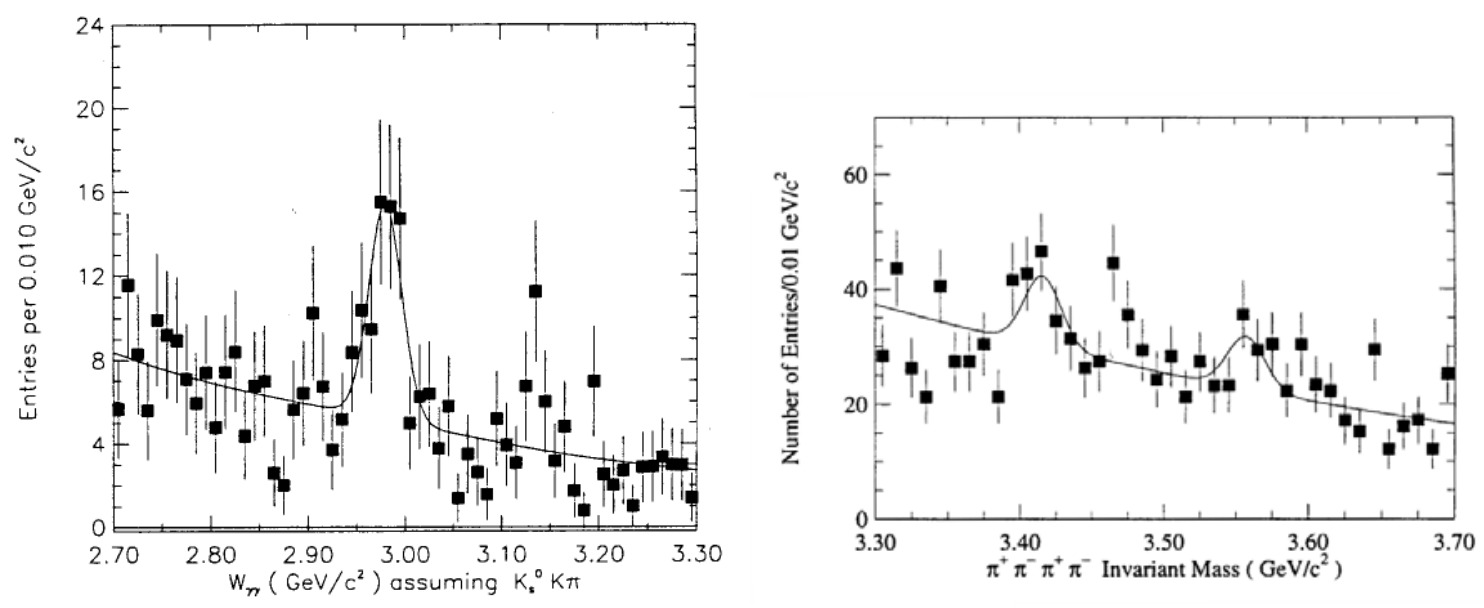

Figure 8.16: Invariant mass spectra from CLEO [66], showing their evidence for (left) $\eta_{c}$ and (right) $\chi_{0}$ and $\chi_{2}$ produced in $\gamma \gamma$ fusion.

fraction has been measured to better than $\sim \pm 30 \%$. Nevertheless, we wish to review the results from these experiments.

Because $\eta_{c}$ is the ground state of charmonium, all hadronic decays are forbidden by the Zweig rule, and they have small branching ratios. Photon-photon fusion experiments must detect $\eta_{c}$ in these weak and poorly-measured hadronic decay channels. Before E760, there were no reported measurements of $\Gamma\left(\gamma \gamma \rightarrow \eta_{c}\right)$ which had more than $\sim 20$ events in a single hadronic decay mode. Since then, single-channel statistics have improved. We consider the most recent $\gamma \gamma$ measurement, reported by the L3 experiment at CERN at ICHEP '98 [74] in some detail here.

The L3 collaboration reported a measurement of $\Gamma\left(\eta_{c} \rightarrow \gamma \gamma\right)=9.5_{-1.9}^{+2.1} \pm 2.7 \mathrm{keV}$ based on the observation of $108 \pm 21$ events spread over ten hadronic decay channels. Of these ten channels, five did not show any excess above the expected background in the $\eta_{c}$ mass region. We reproduce the L3 results in Table 8.6 
Table 8.6: Summary of experimental results for $\eta_{c}$ produced in $\gamma \gamma$ fusion at the L3 experiment.

\begin{tabular}{|lll|}
\hline Channel & $N_{e v t}$ & $\Gamma\left(\eta_{c} \rightarrow \gamma \gamma\right)$ \\
\hline$\eta_{c} \rightarrow \pi^{+} \pi^{-} \pi^{+} \pi^{-}$ & $47.5_{-14.5}^{+16.1} \pm 4.9$ & $8.6_{-2.6}^{+2.9} \mathrm{keV}$ \\
$\eta_{c} \rightarrow K^{0} K^{ \pm} \pi^{\mp}$ & $15.8_{-6.2}^{+6.6} \pm 1.1$ & $7.5_{-2.9}^{+3.2} \mathrm{keV}$ \\
$\eta_{c} \rightarrow \eta(\rightarrow \gamma \gamma) \pi^{+} \pi^{-}$ & $5.4_{-2.1}^{+2.6} \pm 0.08$ & $9.4_{-3.6}^{+4.5} \mathrm{keV}$ \\
$\eta_{c} \rightarrow \pi^{0} \mathrm{~K}^{+} \mathrm{K}^{-}$ & $13.8_{-5.4}^{+6.5} \pm 0.9$ & $31_{-15}^{+16} \mathrm{keV}$ \\
$\eta_{c} \rightarrow \eta^{\prime}(\rightarrow \rho \gamma) \pi^{+} \pi^{-}$ & $25.1_{-8.2}^{+9.1} \pm 2.8$ & $48.8_{-16.0}^{+17.8} \mathrm{keV}$ \\
\hline$\eta_{c} \rightarrow \mathrm{K}^{+} \mathrm{K}^{-} \pi^{+} \pi^{-}$ & $<34.5$ & - \\
$\eta_{c} \rightarrow \rho^{+} \rho^{-}$ & $<12.5$ & - \\
$\eta_{c} \rightarrow \phi \phi$ & $<7.8$ & - \\
$\eta_{c} \rightarrow \eta^{\prime}(\rightarrow \eta \pi \pi)$ & $<4.7$ & - \\
$\eta_{c} \rightarrow \eta(\rightarrow 3 \pi)$ & $<4.2$ & - \\
\hline overall & $\sim 108 \pm 21$ & $9.5_{-1.9}^{+2.1} \pm 1.7$ \\
\hline
\end{tabular}

There are several difficulties which all $\gamma \gamma$ fusion experiments face. First, the reconstruction of $\eta_{c}$ in hadronic decay channels requires the detection of several low-mass and low $p_{T}$ hadrons, and/or photons, while the detectors (like L3) are optimized for looking at high $p_{T}$ particles. Related to this is the problem of determining trigger efficiencies for these low- $p_{T}$ events. Finally, because $\eta_{c}$ is observed in several decay channels, the efficiencies and acceptances for event selection and reconstruction for all the channels need to be determined accurately. This is a daunting task.

To summarize, we note that, despite their relatively large errors, the results from the three $\bar{p} p$ annhilation experiments, E835, E760 and R704, are consistent - their average is $5.6 \pm 1.8 \mathrm{keV}$. Other experimental results have errors of $\sim 40 \%$ each, but are also consistent, with the present world average being $\Gamma\left(\eta_{c} \rightarrow \gamma \gamma\right)=6.3 \pm 1.0 \mathrm{keV}$. 


\subsection{The Two Photon Partial Width of $\chi_{2}$}

E760 reported a measurement of $\Gamma\left(\chi_{2} \rightarrow \gamma \gamma\right)=0.321 \pm 0.078 \pm 0.054$ [71] which differed significantly from the previous world average. [72] Most of the previous determinations of the $\gamma \gamma$ partial width of $\chi_{2}$ came from $\gamma \gamma$-fusion experiments, such as CLEO, [73][66] and all these measurements appeared to be consistent with each other, and much larger (albeit with large statistical errors) than the E760 result. It was therefore considered important to measure $\Gamma\left(\chi_{2} \rightarrow \gamma \gamma\right)$ again.

We have made a measurement of $\Gamma\left(\chi_{2} \rightarrow \gamma \gamma\right)$ in E835 with approximately $8.4 p b^{-1}$ of integrated luminosity near the $\chi_{2}$ peak, compared to $\sim 2.6 p b^{-1}$ in E760. The cross sections for $\bar{p} p \rightarrow \gamma \gamma$ in this region are shown in Fig. 8.17. The total width $\Gamma\left(\chi_{2}\right)=1.98 \pm 0.18 \mathrm{MeV}$, the $\bar{p} p$ branching fraction $B\left(\chi_{2} \rightarrow \bar{p} p\right)=1.0 \pm 0.1 \times 10^{-4}$ and the mass $M\left(\chi_{2}\right)=3556.16 \pm 0.14 \mathrm{MeV}$ were each well measured in E760 in the reaction $\bar{p} p \rightarrow \chi_{2} \rightarrow \gamma J / \psi$. In the present analysis we have fixed $\Gamma\left(\chi_{2}\right)$ and $B\left(\chi_{2} \rightarrow \bar{p} p\right)$ to the above values. To allow for errors in the overall energy scale determined by our beam momentum measurements, we have allowed the mass $M\left(\chi_{2}\right)$ to vary in the fit.

From the fit to our data, we obtain

$$
B\left(\chi_{2} \rightarrow \bar{p} p\right) \times B\left(\chi_{2} \rightarrow \gamma \gamma\right)=1.72 \pm 0.26 \pm 0.04 \times 10^{-8}
$$

or, equivalently,

$$
B\left(\chi_{2} \rightarrow \bar{p} p\right) \times \Gamma\left(\chi_{2} \rightarrow \gamma \gamma\right)=0.0343_{-0.0051}^{+0.0053} \pm 0.021 \mathrm{eV}
$$

The first errors are statistical ${ }^{\dagger}$ and the second are systematic errors obtained by

\footnotetext{
${ }^{\dagger}$ The errors determined by the fit, and by Monte Carlo evaluation (see Appendix D) are iden-
} 


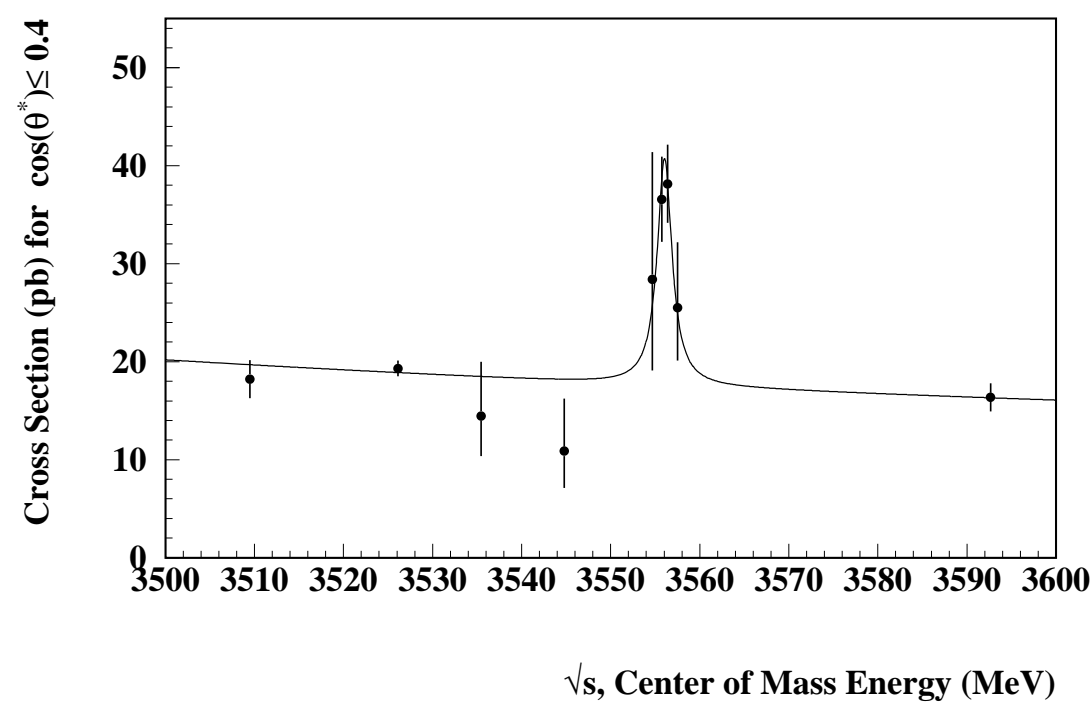

Figure 8.17: Measured cross sections for $\bar{p} p \rightarrow \gamma \gamma$ for $\left|\cos \theta^{*}\right| \leq 0.4$ in the $\chi_{2}$ region.

Table 8.7: Experimental results from E760 and E835 for $\chi_{2}$. In both E760 and E835 analyses, the total width $\Gamma\left(\chi_{2}\right)$ and the branching fraction $B\left(\chi_{2} \rightarrow \bar{p} p\right)$ were fixed to the values determined by E760 from analysis of the reaction $\bar{p} p \rightarrow \chi_{2} \rightarrow \gamma J / \psi$.

\begin{tabular}{|l|ll|}
\hline & E760 & E835 \\
\hline Mass $(\mathrm{MeV})$ & $3556.16 \pm 0.14$ & $3556.10 \pm 0.35$ \\
$B\left(\chi_{2} \rightarrow \bar{p} p\right) \times B\left(\chi_{2} \rightarrow \gamma \gamma\right) \times 10^{8}$ & $1.60 \pm 0.39 \pm 0.16^{*}$ & $1.72 \pm 0.26 \pm 0.04$ \\
$B\left(\chi_{2} \rightarrow \bar{p} p\right) \times \Gamma\left(\chi_{2} \rightarrow \gamma \gamma\right)(\mathrm{eV})$ & $0.032 \pm 0.008 \pm 0.004$ & $0.0343_{-0.0051}^{+0.0053} \pm 0.021$ \\
\hline$B\left(\chi_{2} \rightarrow \bar{p} p\right) \times 10^{4}$ & $1.0 \pm 0.1^{\dagger}$ & $1.0 \pm 0.1^{\dagger}$ \\
\hline$B\left(\chi_{2} \rightarrow \gamma \gamma\right) \times 10^{4}$ & $1.6 \pm 0.4 \pm 0.2$ & $1.72 \pm 0.26_{-0.16}^{+0.19}$ \\
$\Gamma\left(\chi_{2} \rightarrow \gamma \gamma\right)(\mathrm{keV})$ & $0.321 \pm 0.078 \pm 0.054$ & $0.343_{-0.051-0.038}^{+0.053+0.043}$ \\
\hline Signal Events ${ }^{\ddagger}$ & 30 & 87 \\
\hline
\end{tabular}

*The second error given is an estimate of the contribution due to background subtraction.

$\dagger$ Assumed

$\ddagger$ Events in $3554 \leq \sqrt{s}(\mathrm{MeV}) \leq 3558$ region. 
varying the 'fixed' $\Gamma\left(\chi_{2}\right)$ between the experimental errors in the fit. Dividing these by the PDG value for the branching fraction $B\left(\chi_{2} \rightarrow \bar{p} p\right)=1.0 \pm 0.1 \times 10^{-4}$, we find

$$
B\left(\chi_{2} \rightarrow \gamma \gamma\right)=1.72 \pm 0.26_{-0.16}^{+0.19} \times 10^{-4}
$$

and

$$
\Gamma\left(\chi_{2} \rightarrow \gamma \gamma\right)=0.343_{-0.051-0.038}^{+0.053+0.043} \mathrm{keV}
$$

Here, the systematic error quoted is the combination of the $10 \%$ uncertainty on $B\left(\chi_{2} \rightarrow \bar{p} p\right)$ with the systematic errors in Eq. 8.18 and 8.19. Thus the present measurement confirms the E760 results, and have $\gtrsim 30 \%$ smaller errors. (see Table 8.7)

If we fit the data allowing the total width $\Gamma\left(\chi_{2}\right)$ to vary as well, we obtain $M\left(\chi_{2}\right)=3556.07_{-0.43}^{+0.45} \mathrm{MeV}, \Gamma\left(\chi_{2}\right)=2.81_{-1.10}^{+2.65}$, and $B\left(\chi_{2} \rightarrow \bar{p} p\right) \times B\left(\chi_{2} \rightarrow \gamma \gamma\right)=$ $1.57_{-0.29}^{+0.36} \times 10^{-8}$. We note that despite the poor determination of width because of the lack of data in the wings of the resonance, the branching fraction is very well determined, and agrees with our final results, which were obtained by fixing the width.

From the $\chi_{2}$ best fit, for $\left|\cos \theta^{*}\right| \leq 0.4$, the background parameters are found to be

$$
\begin{gathered}
A=311.5_{-25.0}^{+16.4} \mathrm{pb} \quad B=24.0_{-1.8}^{+2.2} \\
C=13.0_{-2.2}^{+1.9} \mathrm{pb} \quad D=2.2_{-3.7}^{+3.6}
\end{gathered}
$$

These background parameters were obtained by fitting all the data from $\sqrt{s}=2911$ to $3829 \mathrm{MeV}$. They will be used again to define the background for our analysis of the data in the $\chi_{0}$ and $\eta_{c}^{\prime}$ regions.

tical. 


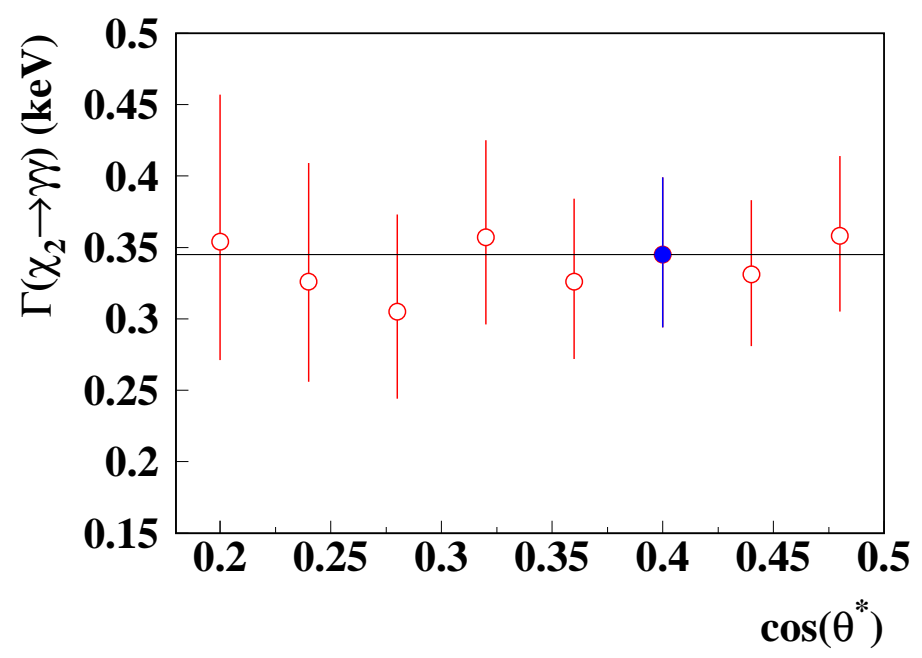

Figure 8.18: Results of a study of the stability of the result for $\Gamma\left(\chi_{2} \rightarrow \gamma \gamma\right)$ relative to the choice of $\left|\cos \theta^{*}\right|$ cut value.

The stability of our present result was checked with respect to the choice of $\cos \left(\theta^{*}\right)$ cut by varying the value of the cut in 8 points from 0.2 to 0.48 and evaluating the partial width. As shown in Fig. 8.18, the result is quite stable.

Our measurement of $\Gamma\left(\chi_{2} \rightarrow \gamma \gamma\right)$ is also shown for comparison with other experimental results in Fig. 8.19 and Table 8.8. Our result is, like that obtained by E760, significantly lower and much more precise than the results from $\gamma \gamma$ fusion measurements. 


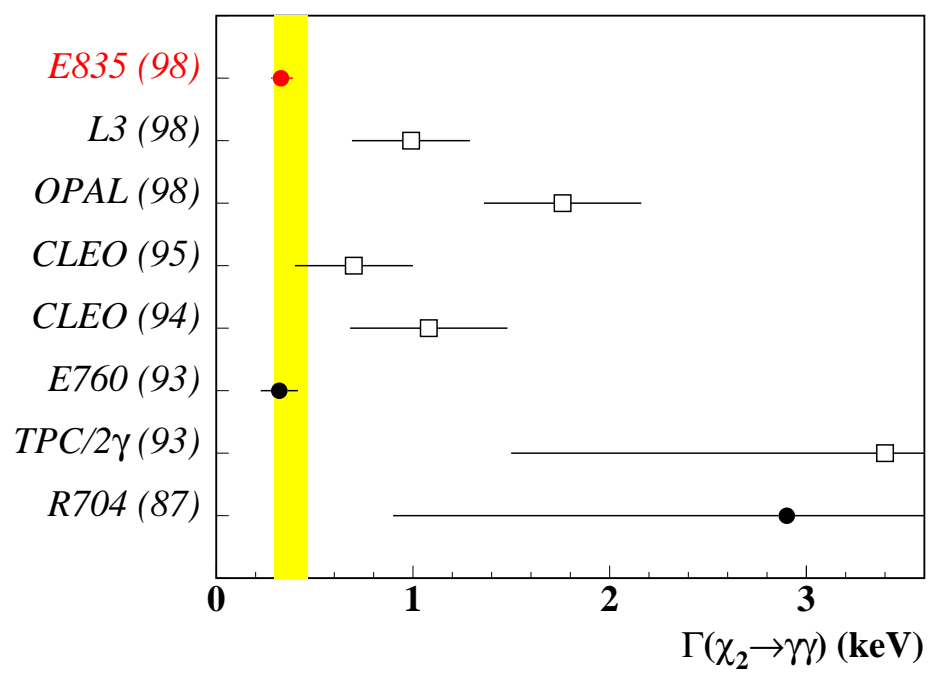

Figure 8.19: Experimental measurements of $\Gamma\left(\chi_{2} \rightarrow \gamma \gamma\right)$, for comparison to the E835 result. The shaded band indicates the weighted average of the displayed results. The open squares indicate results from $\gamma \gamma$ fusion experiments. The solid circles indicate results from $\bar{p} p$ annihilation experiments.

Table 8.8: Experimental results for $\Gamma\left(\chi_{2} \rightarrow \gamma \gamma\right)$. The weighted averages presented have been calculated according to the method prescribed in PDG98. [2]

\begin{tabular}{lllll}
\hline Result (keV) & Experiment (year) & Events & Measurement & Reference \\
\hline $0.343_{-0.051-0.038}^{+0.053+0.043}$ & E835(99) & 87 & $\bar{p} p \rightarrow \gamma \gamma$ & \\
$1.02 \pm 0.4 \pm 0.17$ & L3(98) & 12 & $\gamma \gamma \rightarrow \gamma J / \psi$ & {$[74]$} \\
$1.76 \pm 0.47 \pm 0.40$ & OPAL(98) & 22 & $\gamma \gamma \rightarrow \gamma J / \psi$ & {$[75]$} \\
$0.7 \pm 0.2 \pm 0.2$ & CLEO(95) & 42 & $\gamma \gamma \rightarrow 2\left(\pi^{+} \pi^{-}\right)$ & {$[66]$} \\
$1.08 \pm 0.30 \pm 0.26$ & CLEO(94) & 25 & $\gamma \gamma \rightarrow \gamma J / \psi$ & {$[73]$} \\
$0.321 \pm 0.078 \pm 0.054$ & E760 $(93)[50]$ & 30 & $\bar{p} p \rightarrow \gamma \gamma$ & \\
$3.4 \pm 1.7 \pm 0.9$ & TPC/2 $\gamma(93)$ & 6 & $\gamma \gamma \rightarrow \gamma J / \psi$ & {$[76]$} \\
$2.9_{-1.0}^{+1.3} \pm 1.7$ & R704(87) & 6 & $\bar{p} p \rightarrow \gamma \gamma$ & {$[61]$} \\
\hline $0.38 \pm 0.08$ & Weighted Average & & \\
\hline
\end{tabular}




\subsection{Measurement of $\bar{p} p \rightarrow \chi_{0} \rightarrow \gamma \gamma$}

The $\chi_{0}$ resonance has been observed by us in the reaction $\bar{p} p \rightarrow \gamma J / \psi$, and the results for the $\chi_{0}$ resonance parameters from that measurement are: $[77,78]$

$$
\begin{array}{r}
M\left(\chi_{0}\right)=3417.4_{-1.9}^{+1.8} \pm 0.3 \mathrm{MeV} \\
\Gamma\left(\chi_{0}\right)=16.6_{-3.7}^{+5.2} \pm 0.1 \mathrm{MeV} \\
B\left(\chi_{0} \rightarrow \bar{p} p\right)=4.82_{-0.78}^{+0.94} \pm 0.23\left(_{-1.12}^{+2.08}\right) \times 10^{-4}
\end{array}
$$

Since $\Gamma\left(\chi_{0} \rightarrow \gamma \gamma\right)$ is expected to be larger than $\Gamma\left(\chi_{2} \rightarrow \gamma \gamma\right)$, we ought to be able to observe $\chi_{0}$ in the reaction $\bar{p} p \rightarrow \chi_{0} \rightarrow \gamma \gamma$. However, we have failed to find evidence for $\chi_{0}$ in this reaction, largely because of the small value of the luminosity invested in the $\chi_{0}$ measurements. The measured $\gamma \gamma$ cross sections for $\left|\cos \theta^{*}\right| \leq 0.4$ are plotted for energies between 3200 and $3800 \mathrm{MeV}$ in Fig. 8.20. The curve shows the background fit for $\left|\cos \theta^{*}\right| \leq 0.4$, obtained in Eq. 8.22. It is clear that we do not have sufficient statistics, to claim evidence for $\chi_{0}$ in these data, but we can set an upper limit on the product $B\left(\chi_{0} \rightarrow \bar{p} p\right) \times \Gamma\left(\chi_{0} \rightarrow \gamma \gamma\right)$.

The $\gamma \gamma$ data shown in Fig. 8.20 have been fitted for the product $B\left(\chi_{0} \rightarrow \bar{p} p\right) \times$ $\Gamma\left(\chi_{0} \rightarrow \gamma \gamma\right)$, with the mass and total width fixed to the measured values in Eq. 8.23. The background is also fixed to the values presented in Sec. 8.4. Using the method outlined in Appendix D, we have determined a 90\% confidence upper limit for $B\left(\chi_{0} \rightarrow \bar{p} p\right) \times \Gamma\left(\chi_{0} \rightarrow \gamma \gamma\right):$

$$
B\left(\chi_{0} \rightarrow \bar{p} p\right) \times \Gamma\left(\chi_{0} \rightarrow \gamma \gamma\right) \leq 0.63 \mathrm{eV}, \quad 90 \% C L
$$

Dividing this by $B\left(\chi_{0} \rightarrow \bar{p} p\right)$ from Eq. 8.23, we find that

$$
\Gamma\left(\chi_{0} \rightarrow \gamma \gamma\right) \leq 1.3 \mathrm{keV}, \quad 90 \% C L
$$




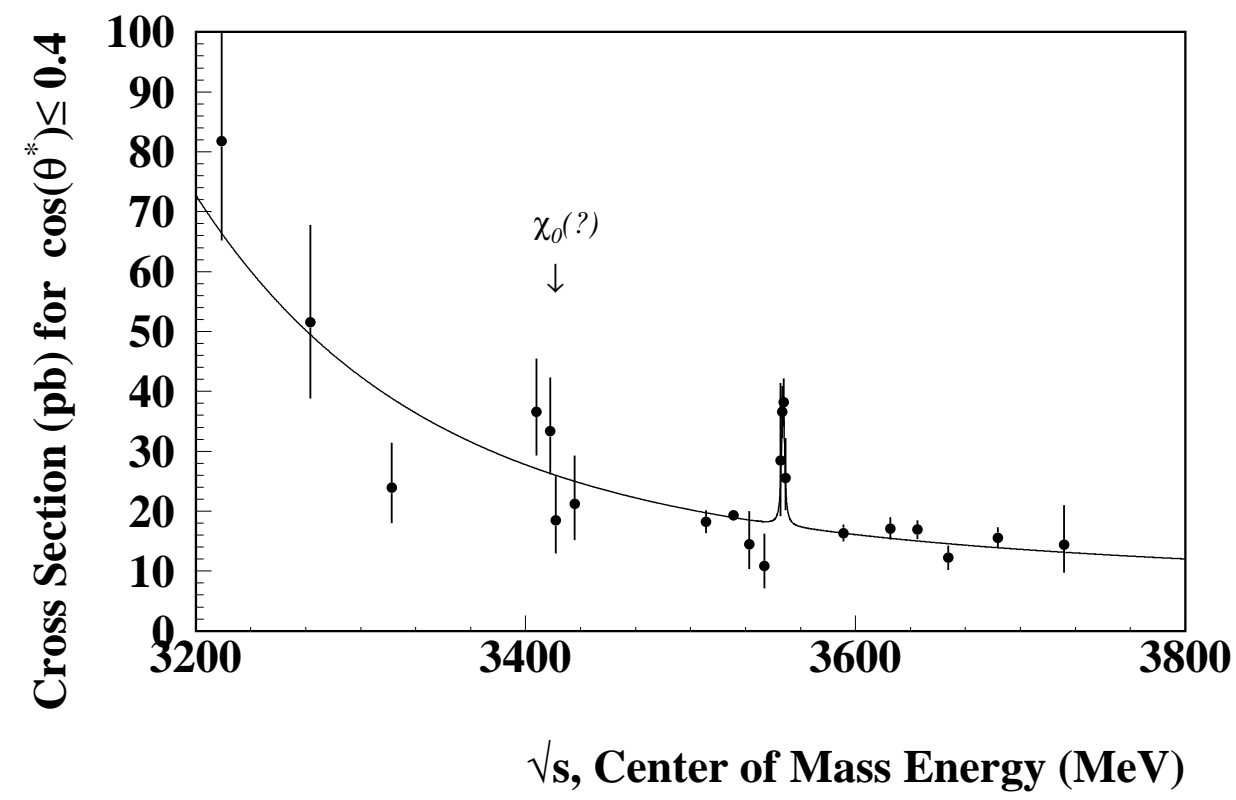

Figure 8.20: Measured cross sections for $\bar{p} p \rightarrow \gamma \gamma$ for $\left|\cos \theta^{*}\right| \leq 0.4$ in the $\chi_{0}$ search region. The solid line is the result of the fit to the background, with the parameters presented in Sec. 8.4. The $\chi_{2}$ peak at $\sqrt{s}=3556.18 \mathrm{MeV}$ is clearly visible. The E835 result for the $\chi_{0}$ mass, $M\left(\chi_{0}\right)=3417.4_{-1.9}^{+1.8} \pm 0.3 \mathrm{MeV}$, measured in the reaction $\bar{p} p \rightarrow \chi_{0} \rightarrow \gamma J / \psi$, is indicated by the arrow.

This upper limit may be compared with the two measurements of the partial width in the literature, $\left.\Gamma\left(\chi_{0} \rightarrow \gamma \gamma\right)=4.2 \pm 2.8 \mathrm{keV}\right)$ from the Crystal Ball [79] and $\Gamma\left(\chi_{0} \rightarrow \gamma \gamma\right)=1.7 \pm 0.8 \mathrm{keV}$, from CLEO. [80]. The CLEO spectrum is displayed in Fig. 8.16. 


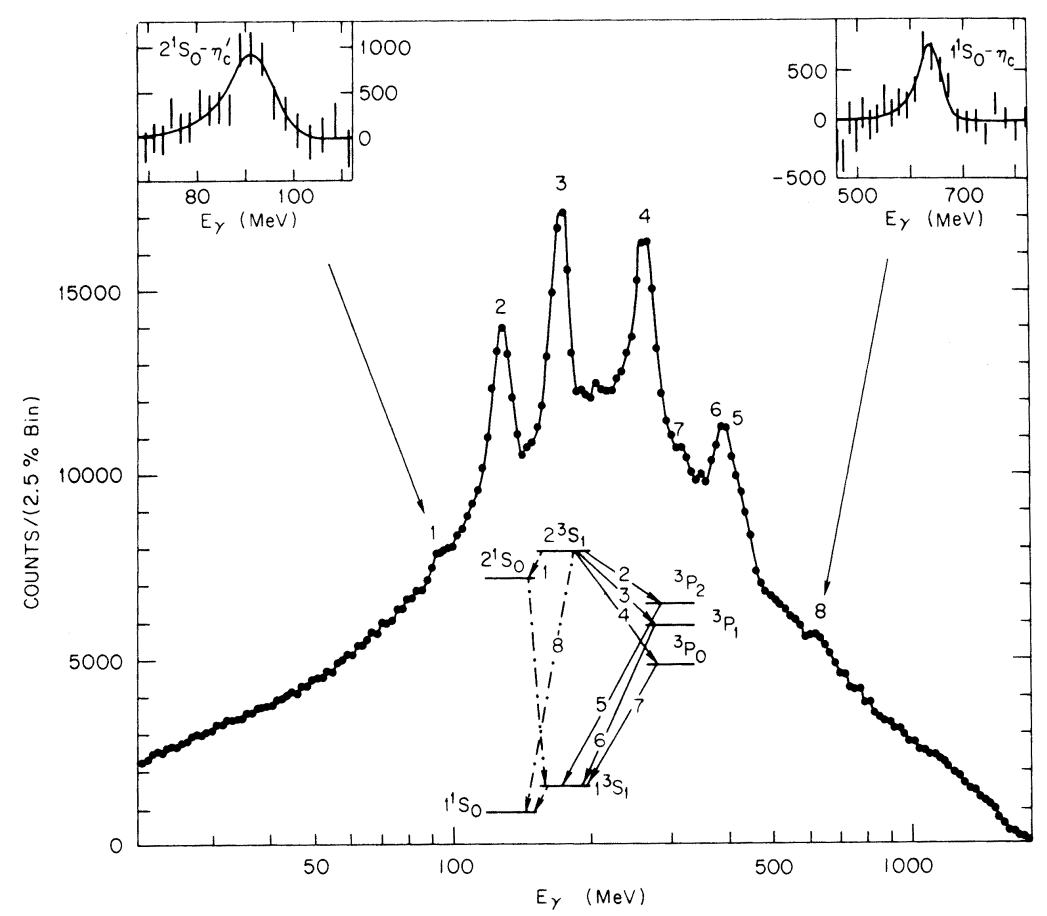

Figure 8.21: Evidence for $\eta_{c}^{\prime}$ in the inclusive photon spectrum from the Crystal Ball. [81] The insets in the upper left and upper right hand parts of the figure are expanded views of the background-subtracted spectrum in the regions corresponding to transitions to $\eta_{c}^{\prime}$ and $\eta_{c}$.

\subsection{The Search for $\eta_{c}^{\prime}$}

The radial excitation of $\eta_{c}\left(1^{1} S_{0}\right)$, the ground state of charmonium, is $\eta_{c}^{\prime}\left(2^{1} S_{0}\right)$. Its identification has been reported by only one experiment, the Crystal Ball experiment at SLAC. The Crystal Ball observed an excess of events in the inclusive photon spectrum from $\psi^{\prime}[81]$ at a mean photon energy of $91 \pm 5 \mathrm{MeV}$, which corresponds to a mass of $M\left(\eta_{c}^{\prime}\right)=3594 \pm 5 \mathrm{MeV}$. (see Fig. 8.21.) The Crystal Ball also reported a $95 \%$ confidence level upper limit for the total width $\Gamma\left(\eta_{c}^{\prime}\right)$ of $8 \mathrm{MeV}$. These results 
for $\eta_{c}^{\prime}$ are not included in the PDG summary tables for lack of any confirming measurements. E760 attempted to identify $\eta_{c}^{\prime}$, but failed. [50] We describe here the results of our search.

\section{Predictions for $\eta_{c}^{\prime}$ mass and width}

The choice of the E760/E835 search region for $\eta_{c}^{\prime}$, and the spacing between data points in $\sqrt{s}$ depended not only on the mass and width of the Crystal Ball candidate, but on theoretical estimates of the same. We discuss here theoretical predictions for the $\eta_{c}^{\prime}$ mass and width which motivated the search in E760 and E835.

\section{$\underline{\text { Predictions for } M\left(\eta_{c}^{\prime}\right)}$}

Buchmüller and Tye [83] have shown that for $l=0$, one obtains the familiar form of the hyperfine splitting in a Coulomb potential [1] with only an additional factor for the QCD radiative correction:

$$
\Delta M\left({ }^{3} S_{1}-{ }^{1} S_{0}\right)=\frac{32 \pi}{9} \frac{\alpha_{\mathrm{S}}}{m_{c}^{2}}|\psi(0)|^{2}\left[1+0.77 \frac{\alpha_{\mathrm{S}}}{\pi}\right] .
$$

To the same order,

$$
\Gamma\left(V \rightarrow e^{+} e^{-}\right)=\frac{16 \pi}{9} \frac{\alpha_{\mathrm{EM}}^{2}}{m_{c}^{2}}|\psi(0)|^{2}\left[1-5.33 \alpha_{\mathrm{S}} / \pi\right] .
$$

Thus,

$$
\Delta M\left({ }^{3} S_{1}-{ }^{1} S_{0}\right)=2 \Gamma\left(V \rightarrow e^{+} e^{-}\right) \times \frac{\alpha_{\mathrm{S}}}{\alpha_{\mathrm{EM}}{ }^{2}}\left[\frac{1+0.77 \alpha_{\mathrm{S}} / \pi}{1-5.33 \alpha_{\mathrm{S}} / \pi}\right] .
$$

It follows, therefore, that

$$
\frac{\Delta M\left(\psi^{\prime}-\eta_{c}^{\prime}\right)}{\Delta M\left(J / \psi-\eta_{c}\right)}=\frac{\Gamma\left(\psi^{\prime} \rightarrow e^{+} e^{-}\right)}{\Gamma\left(J / \psi \rightarrow e^{+} e^{-}\right)}=\frac{2.14 \pm 0.21 \mathrm{keV}}{5.26 \pm 0.38 \mathrm{keV}}=0.407 \pm 0.049
$$


We note that sometimes $m_{c}$ in Eq.8.27 is replaced by $\left(M_{V} / 2\right)$, while retaining $m_{c}$ in Eq. 8.26. This has an historical origin (the original formulation by Van Royen and Weisskopf [22]) and is obviously inconsistent. It gives rise to an additional multiplicative factor of 1.416 in Eq. 8.28, so that

$$
\frac{\Delta M\left(\psi^{\prime}-\eta_{c}^{\prime}\right)}{\Delta M\left(J / \psi-\eta_{c}\right)}=0.576 \pm 0.070 .
$$

With our result of $\Delta M\left(J / \psi-\eta_{c}\right)=114.5 \mathrm{MeV}$, Eq. 8.29 leads to $\Delta M\left(\psi^{\prime}-\eta_{c}^{\prime}\right)=$ $46.6 \pm 5.7 \mathrm{MeV}, M\left(\eta_{c}^{\prime}\right)=3639 \pm 6 \mathrm{MeV}$, and Eq. 8.30 leads to $\Delta M\left(\psi^{\prime}-\eta_{c}^{\prime}\right)=$ $66.0 \pm 8.0 \mathrm{MeV}, M\left(\eta_{c}^{\prime}\right)=3620 \pm 8 \mathrm{MeV}$.

Potential model calculations do not rely on relations such as Eqs. 8.26 and 8.27. They assume potential forms, $m_{c}$ and $\alpha_{\mathrm{S}}$ to fit the masses of one or more charmonium levels, and predict masses and wave functions for various charmonium states. Several such calculations exist in the literature.

Gupta etal. [84] use a non-singular $q \bar{q}$ potential with a scalar-vector form of the confining potential, and obtain excellent agreement with the masses of all wellestablished charmonium states $\left(J / \psi, \eta_{c}, \psi^{\prime}, \chi_{J}, h_{c}\right.$, all within $\left.\pm 5 \mathrm{MeV}\right)$. They predict $M\left(\eta_{c}\right)=3622.3 \mathrm{MeV}$.

Zhu etal. [85] report a relativistic calculation in the Bethe-Salpeter formalism. They fit $J / \psi, \psi^{\prime}, \psi^{(2)}$ and $\eta_{c}$ masses within $\pm 3 \mathrm{MeV}$, and predict $M\left(\eta_{c}^{\prime}\right)=3634$ $\mathrm{MeV}$.

Eichten and Quigg [86] have made numerical calculations using several different types of potentials (Buchmüller-Tye, Martin, logarithmic, Cornell) with parameters chosen to reproduce the $M(J / \psi)-M\left(\eta_{c}\right)$ splitting. Their results (renormalized by $2 \%$ to account for our determination of $\left.M\left(\eta_{c}\right)\right)$ average to give $M\left(\eta_{c}^{\prime}\right)=3618 \pm 8$ 
MeV. Chen and Oakes [87] make an investigation similar to that of Eichten and Quigg with Cornell, logarithmic, and their own 'renormalization-group-improved' potential, and obtain a very similar prediction, $M\left(\eta_{c}^{\prime}\right)=3620_{-12}^{+9} \mathrm{MeV}$.

A fair summary of the above is that all recent calculations indicate that $M\left(\eta_{c}^{\prime}\right) \approx$ $3630 \pm 10 \mathrm{MeV}$. This is $\sim 35 \mathrm{MeV}$ larger than the mass of $3594 \pm 5 \mathrm{MeV}$ reported by the Crystal Ball experiment. [81] This sets the stage for our search for $\eta_{c}^{\prime}$. In the present investigation we scanned the mass region from 3576 to $3660 \mathrm{MeV}$ in order to identify $\eta_{c}^{\prime}$.

Estimates for $\Gamma\left(\eta_{c}^{\prime}\right)$

A close correspondence between $\psi^{\prime}$ and $\eta_{c}^{\prime}$ can be drawn as far as the nonannihilation decay channels are concerned. Thus,

$$
\begin{aligned}
a) \Gamma\left(\eta_{c}^{\prime} \rightarrow \gamma(E 1)+{ }^{1} P_{1}\right) & \rightarrow \Sigma_{J} \Gamma\left(\psi^{\prime} \rightarrow \gamma(E 1)+{ }^{3} P_{J}\right) \approx 80 \mathrm{keV} \\
b) \Gamma\left(\eta_{c}^{\prime} \rightarrow \eta_{c}+X\right) & \rightarrow \Gamma\left(\psi^{\prime} \rightarrow J / \psi+X\right) \approx 170 \mathrm{keV} \\
c) \Gamma\left(\eta_{c}^{\prime} \rightarrow{ }^{3} P_{0}+\pi^{0}\right) & \rightarrow \Gamma\left(\psi^{\prime} \rightarrow{ }^{1} P_{1}+\pi^{0}\right) \leq 1.3 \mathrm{keV}
\end{aligned}
$$

Thus the non-annihilation channels of $\eta_{c}^{\prime}$ are not expected to contribute more than $0.3 \mathrm{MeV}$ to its width.

As far as $c \bar{c}$ annihilation channels are concerned, the ${ }^{1} S_{0}$ states are indeed different from the ${ }^{3} S_{1}$ states in that the ${ }^{1} S_{0}$ 's must annihilate via two gluons, and the ${ }^{3} S_{1}$ 's via three gluons, leading to the fact that the hadronic decay widths of the ${ }^{1} S_{0}$ 's are larger than those of the ${ }^{3} S_{1}$ 's by big factors of the order of 100 . It is therefore not advisable to relate the $\eta_{c}^{\prime}$ hadronic decays directly to those of $\psi^{\prime}$. It appears to 
be more prudent to make the analogy

$$
\frac{\Gamma\left(\eta_{c}^{\prime} \rightarrow g g\right)}{\Gamma\left(\eta_{c} \rightarrow g g\right)}=\frac{\Gamma\left(\psi^{\prime} \rightarrow g g g\right)}{\Gamma(J / \psi \rightarrow g g g)}=\frac{46 \pm 10 \mathrm{keV}}{71 \pm 6 \mathrm{keV}}=0.66 \pm 0.15 .
$$

This ratio is in accord with the ratio of the squares of the wave functions of $\eta_{c}^{\prime}$ and $\eta_{c}$ (as well as $\psi^{\prime}$ and $J / \psi$ ) calculated in the potential models. Eichten and Quigg [86] have calculated the ratio for several potentials, and their average result is

$$
\frac{|\psi(0)|^{2}\left(\eta_{c}^{\prime}\right)}{|\psi(0)|^{2}\left(\eta_{c}\right)}=0.60 \pm 0.05
$$

Using the experimental ratio in Eq. 8.32, and our determination of $\Gamma\left(\eta_{c} \rightarrow g g\right) \approx$ $\Gamma\left(\eta_{c}\right)=26.9_{-9.5}^{+10.8} \mathrm{MeV}$, we obtain the estimate for the $\eta_{c}^{\prime}$ width,

$$
\Gamma\left(\eta_{c}^{\prime}\right) \approx \Gamma\left(\eta_{c}^{\prime} \rightarrow g g\right)=17.8_{-6.3}^{+7.1} \pm 6.1 \mathrm{MeV}
$$

\section{The search for $\eta_{c}^{\prime}$ by E760}

E760 searched for $\eta_{c}^{\prime}$ at 6 data points from 3591 to $3621 \mathrm{MeV}$, They invested a total of $\sim 6 p b^{-1}$ of luminosity in the search, and obtained $90 \%$ confidence level upper limits on the product of branching ratios of [50]

$$
\begin{aligned}
& B\left(\eta_{c}^{\prime} \rightarrow \bar{p} p\right) \times B\left(\eta_{c}^{\prime} \rightarrow \gamma \gamma\right) \leq 14.9 \times 10^{-8}, \text { for assumed } \Gamma\left(\eta_{c}^{\prime}\right)=5 \mathrm{MeV}, \text { and } \\
& B\left(\eta_{c}^{\prime} \rightarrow \bar{p} p\right) \times B\left(\eta_{c}^{\prime} \rightarrow \gamma \gamma\right) \leq 7.0 \times 10^{-8}, \text { for assumed } \Gamma\left(\eta_{c}^{\prime}\right)=10 \mathrm{MeV},
\end{aligned}
$$

for $\eta_{c}^{\prime}$ with a mass anywhere in the range 3584 to $3624 \mathrm{MeV}$. The upper limits for the branching fraction product for the full range are shown as a function of the assumed mass $M\left(\eta_{c}^{\prime}\right)$ and width $\Gamma\left(\eta_{c}^{\prime}\right)$ in Fig. 8.22. 


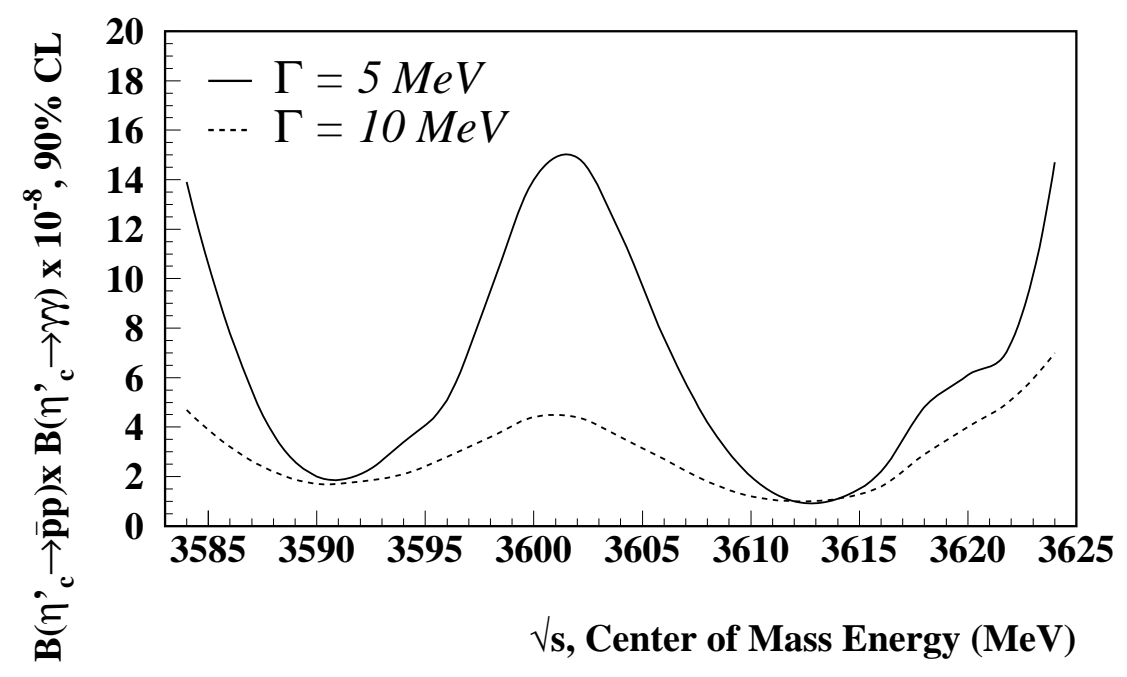

Figure 8.22: $90 \%$ CL upper limits for $B\left(\eta_{c}^{\prime} \rightarrow \bar{p} p\right) \times B\left(\eta_{c}^{\prime} \rightarrow \gamma \gamma\right)$ from E760, [50] for assumed $M\left(\eta_{c}^{\prime}\right)$ in the range 3584 to $3624 \mathrm{MeV}$, and assumed widths of $\Gamma\left(\eta_{c}^{\prime}\right)=5$ and $10 \mathrm{MeV}$.

\section{The search for $\eta_{c}^{\prime}$ by E835}

In experiment E835, we have searched for $\eta_{c}^{\prime}$ in a much wider mass region (3575 to $3660 \mathrm{MeV}$ ), with an investment of $\sim 5$ times greater luminosity, $\sim 30 \mathrm{pb}^{-1}$. The spacing in $\sqrt{s}$ between data points was approximately $5 \mathrm{MeV}$. Despite this marathon effort, once again we have failed to find any evidence for $\eta_{c}^{\prime}$ production. The $\gamma \gamma$ cross sections measured in E835 for $\left|\cos \theta^{*}\right| \leq 0.4$ are presented in Fig. 8.23.

From these data we are only able to derive an upper limit for the branching fraction product $B\left(\eta_{c}^{\prime} \rightarrow \bar{p} p\right) \times B\left(\eta_{c}^{\prime} \rightarrow \gamma \gamma\right)$, for assumed values of the total width. The $\gamma \gamma$ cross sections for $\left|\cos \theta^{*}\right| \leq 0.4$ in the region $3576-3660 \mathrm{MeV}$ were fitted 


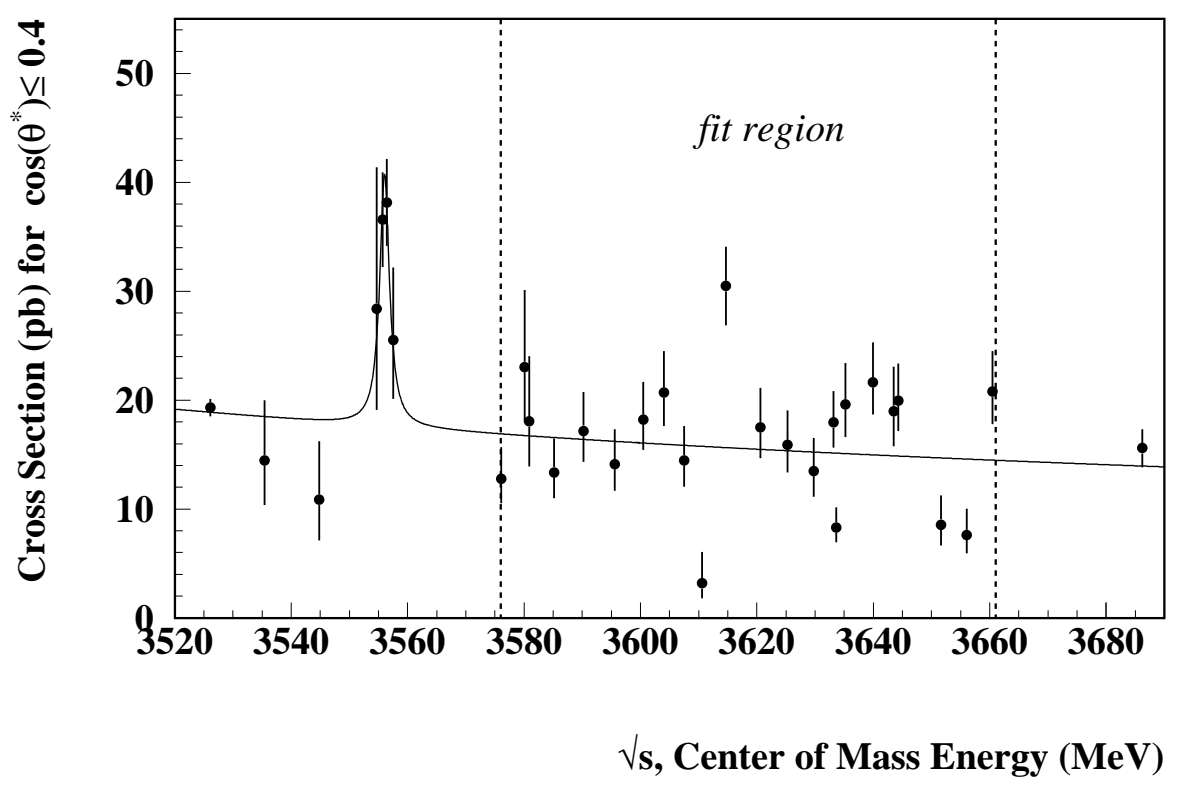

Figure 8.23: Measured cross sections for $\bar{p} p \rightarrow \gamma \gamma$ for $\left|\cos \theta^{*}\right| \leq 0.4$ in the $\eta_{c}^{\prime}$ search region. The $\chi_{2}$ peak at $\sqrt{s}=3556.18 \mathrm{MeV}$ is clearly visible.

for the branching fraction product, with various assumed fixed values of the $\eta_{c}^{\prime}$ mass and total width, (10, 15 and $20 \mathrm{MeV})$ and with the background parameters fixed to the values in Eq.8.22. The assumed mass of $\eta_{c}^{\prime}$ was varied in steps of $1 \mathrm{MeV}$ from 3575 to $3660 \mathrm{MeV}$, and a maximum log likelihood fit was performed at each step. (see Appendix D).

The results of the fits are shown in Fig. 8.24. The summary of our results is that for $\eta_{c}^{\prime}$ masses between 3575 and $3660 \mathrm{MeV}$, the following $90 \%$ confidence upper limits can be set:

$$
B\left(\eta_{c}^{\prime} \rightarrow \bar{p} p\right) \times B\left(\eta_{c}^{\prime} \rightarrow \gamma \gamma\right) \leq 5.9 \times 10^{-8}, \text { for } \Gamma\left(\eta_{c}^{\prime}\right)=10 \mathrm{MeV}, \text { and }
$$




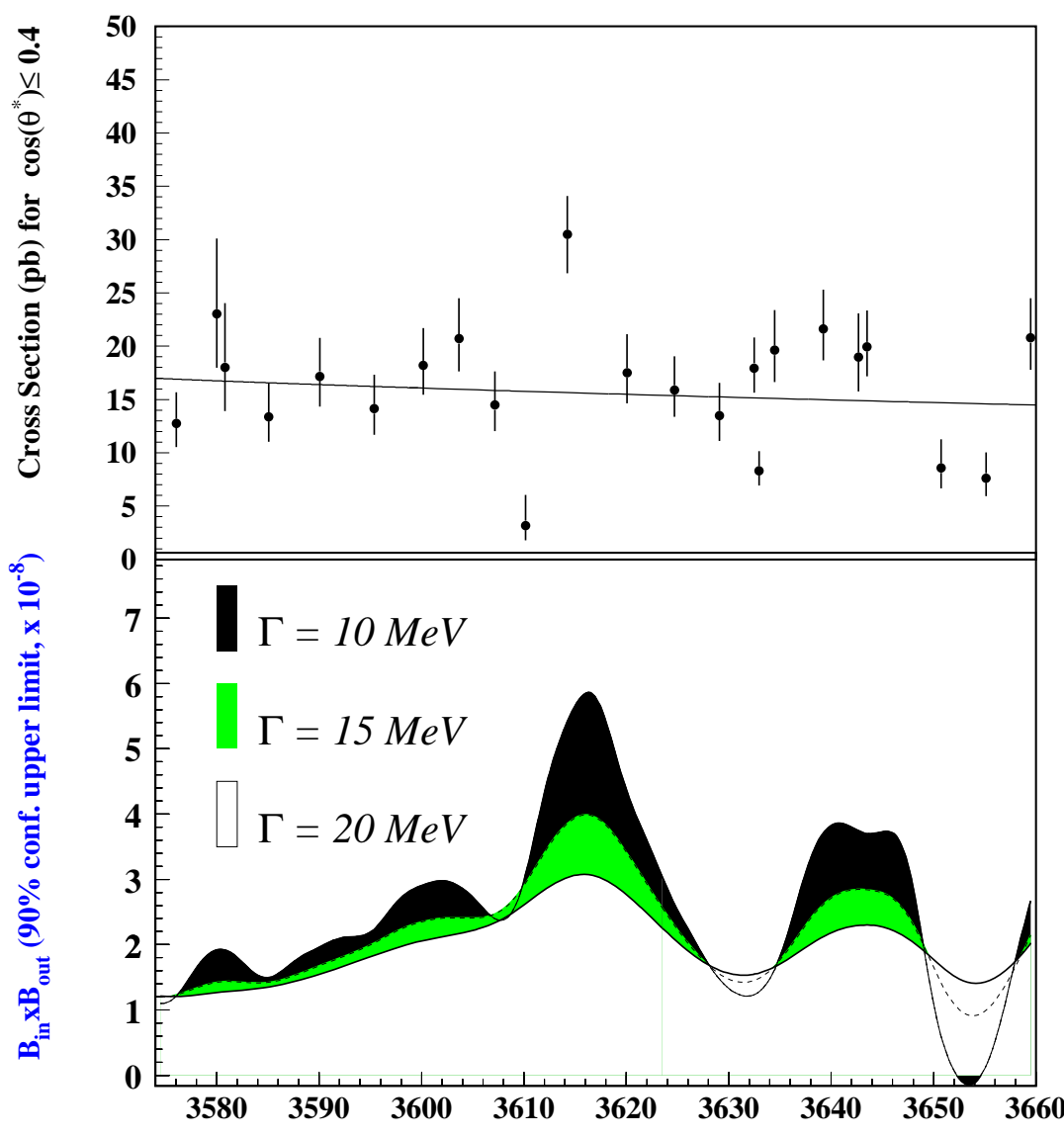

Mass (MeV)

Figure 8.24: (Top) Measured cross sections for $\bar{p} p \rightarrow \gamma \gamma$ for $\left|\cos \theta^{*}\right| \leq 0.4$ in the $\eta_{c}^{\prime}$ search region. (Bottom) Result of maximum likelihood fits to these data. The three curves represent calculations of the $90 \%$ confidence upper limits on $B\left(\eta_{c}^{\prime} \rightarrow \bar{p} p\right) \times$ $B\left(\eta_{c}^{\prime} \rightarrow \gamma \gamma\right)$ for assumed values of $\Gamma\left(\eta_{c}^{\prime}\right)=10,15$ and $20 \mathrm{MeV}$. 


$$
\begin{aligned}
& B\left(\eta_{c}^{\prime} \rightarrow \bar{p} p\right) \times B\left(\eta_{c}^{\prime} \rightarrow \gamma \gamma\right) \leq 4.0 \times 10^{-8}, \text { for } \Gamma\left(\eta_{c}^{\prime}\right)=15 \mathrm{MeV}, \text { and } \\
& B\left(\eta_{c}^{\prime} \rightarrow \bar{p} p\right) \times B\left(\eta_{c}^{\prime} \rightarrow \gamma \gamma\right) \leq 3.1 \times 10^{-8}, \text { for } \Gamma\left(\eta_{c}^{\prime}\right)=20 \mathrm{MeV}
\end{aligned}
$$

The largest of these limits is more than a factor 4 smaller than the corresponding result for $\eta_{c}, B\left(\eta_{c} \rightarrow \bar{p} p\right) \times B\left(\eta_{c} \rightarrow \gamma \gamma\right)=25.3_{-4.5}^{+4.6} \times 10^{-8}$.

\section{Comments on $\eta_{c}^{\prime}$}

We wish to examine the above results in terms of the theoretical and phenomenological expectations.

\section{$\underline{\text { Estimates of } B\left(\eta_{c}^{\prime} \rightarrow \gamma \gamma\right)}$}

The ratio of the lowest order predictions for the $\gamma \gamma$ and $g g$ partial widths of any state of charmonium is independent of the radial quantum number. From Table 2.1, for both $\eta_{c}$ and $\eta_{c}^{\prime}$,

$$
\begin{gathered}
\Gamma\left({ }^{1} S_{0} \rightarrow g g\right)=\frac{8 \pi \alpha_{\mathrm{S}}^{2}}{3 m_{c}^{2}}\left|\psi_{S}(0)\right|^{2}\left(1+4.8 \alpha_{\mathrm{S}} / \pi\right) \\
\Gamma\left({ }^{1} S_{0} \rightarrow \gamma \gamma\right)=\frac{64 \pi \alpha_{\mathrm{EM}}{ }^{2}}{27 m_{c}^{2}}\left|\psi_{S}(0)\right|^{2}\left(1-3.4 \alpha_{\mathrm{S}} / \pi\right)
\end{gathered}
$$

Thus,

$$
\frac{\Gamma\left({ }^{1} S_{0} \rightarrow \gamma \gamma\right)}{\Gamma\left({ }^{1} S_{0} \rightarrow g g\right)}=B\left({ }^{1} S_{0} \rightarrow \gamma \gamma\right)=\frac{8 \alpha_{\mathrm{EM}}{ }^{2}}{9 \alpha_{\mathrm{S}}{ }^{2}} \times \frac{\left(1-3.4 \alpha_{\mathrm{S}} / \pi\right)}{\left(1+4.8 \alpha_{\mathrm{S}} / \pi\right)}
$$

since we have established that both $\eta_{c}$ and $\eta_{c}^{\prime}$ have total widths which are essentially completely hadronic. Therefore, we expect that

$$
B\left(\eta_{c}^{\prime} \rightarrow \gamma \gamma\right) \sim B\left(\eta_{c} \rightarrow \gamma \gamma\right)=\left(2.1 \times 10^{-4}\right)
$$

$\underline{\text { Estimates of } B\left(\eta_{c}^{\prime} \rightarrow \bar{p} p\right)}$ 
The prediction for the branching fraction $B\left(\eta_{c}^{\prime} \rightarrow \bar{p} p\right)$ is somewhat less straightforward. We may assume that the ratio of $\Gamma(c \bar{c} \rightarrow \bar{p} p) / \Gamma(c \bar{c} \rightarrow$ hadrons $)$ between radial excitations of the triplet states $\left(J / \psi\right.$ and $\left.\psi^{\prime}\right)$ is the same as that between the radial excitations of the singlet states $\left(\eta_{c}\right.$ and $\left.\eta_{c}^{\prime}\right)$. i.e.,

$$
\begin{gathered}
\frac{\Gamma\left(\eta_{c}^{\prime} \rightarrow \bar{p} p\right) / \Gamma\left(\eta_{c}^{\prime} \rightarrow \text { hadrons }\right)}{\Gamma\left(\eta_{c} \rightarrow \bar{p} p\right) / \Gamma\left(\eta_{c} \rightarrow \text { hadrons }\right)}=\frac{\Gamma\left(\psi^{\prime} \rightarrow \bar{p} p\right) / \Gamma\left(\psi^{\prime} \rightarrow \text { hadrons }\right)}{\Gamma(J / \psi \rightarrow \bar{p} p) / \Gamma(J / \psi \rightarrow \text { hadrons })} \\
\therefore B\left(\eta_{c}^{\prime} \rightarrow \bar{p} p\right)=B\left(\eta_{c} \rightarrow \bar{p} p\right) \times \frac{\Gamma\left(\psi^{\prime} \rightarrow \bar{p} p\right) / \Gamma\left(\psi^{\prime} \rightarrow \text { hadrons }\right)}{\Gamma(J / \psi \rightarrow \bar{p} p) / \Gamma(J / \psi \rightarrow \text { hadrons })} \\
\therefore B\left(\eta_{c}^{\prime} \rightarrow \bar{p} p\right) \times 10^{4} \\
=(12 \pm 4) \frac{\left(0.19 \pm 0.05 \times 10^{-3}\right) /\left(15.18 \pm 3.36 \times 10^{-2}\right)}{\left(2.14 \pm 0.10 \times 10^{-3}\right) /\left(70.7 \pm 0.6 \times 10^{-2}\right)} \\
=4.9 \pm 2.4 .
\end{gathered}
$$

Combining this with Eq. 8.40, we obtain

$$
B\left(\eta_{c}^{\prime} \rightarrow \bar{p} p\right) \times B\left(\eta_{c}^{\prime} \rightarrow \gamma \gamma\right) \approx 10.3 \pm 5.0 \times 10^{-8}
$$

Thus the experimental limits which we have established in Eq. 8.36 are consistent with these semi-phenomenological expectations.

\section{$\underline{\text { Recent results from DELPHI }}$}

Earlier, (Sec. 8.3.3) we mentioned $\Gamma_{\gamma \gamma}$ obtained for $\eta_{c}$ and $\chi_{2}$ resonances by photon-photon fusion experiments. It is worthwhile to look into what these experiments have to say about $\Gamma_{\gamma \gamma}\left(\eta_{c}^{\prime}\right)$. Actually, only one measurement has been reported so far. The DELPHI collaboration at LEP has recently published a result [98] which may add an alternate perspective on the question of our non-observation of $\eta_{c}^{\prime}$ in the reaction $\bar{p} p \rightarrow \eta_{c}^{\prime} \rightarrow \gamma \gamma$. The usual expectation for the partial width $\Gamma\left(\eta_{c}^{\prime} \rightarrow \gamma \gamma\right)$ is that 


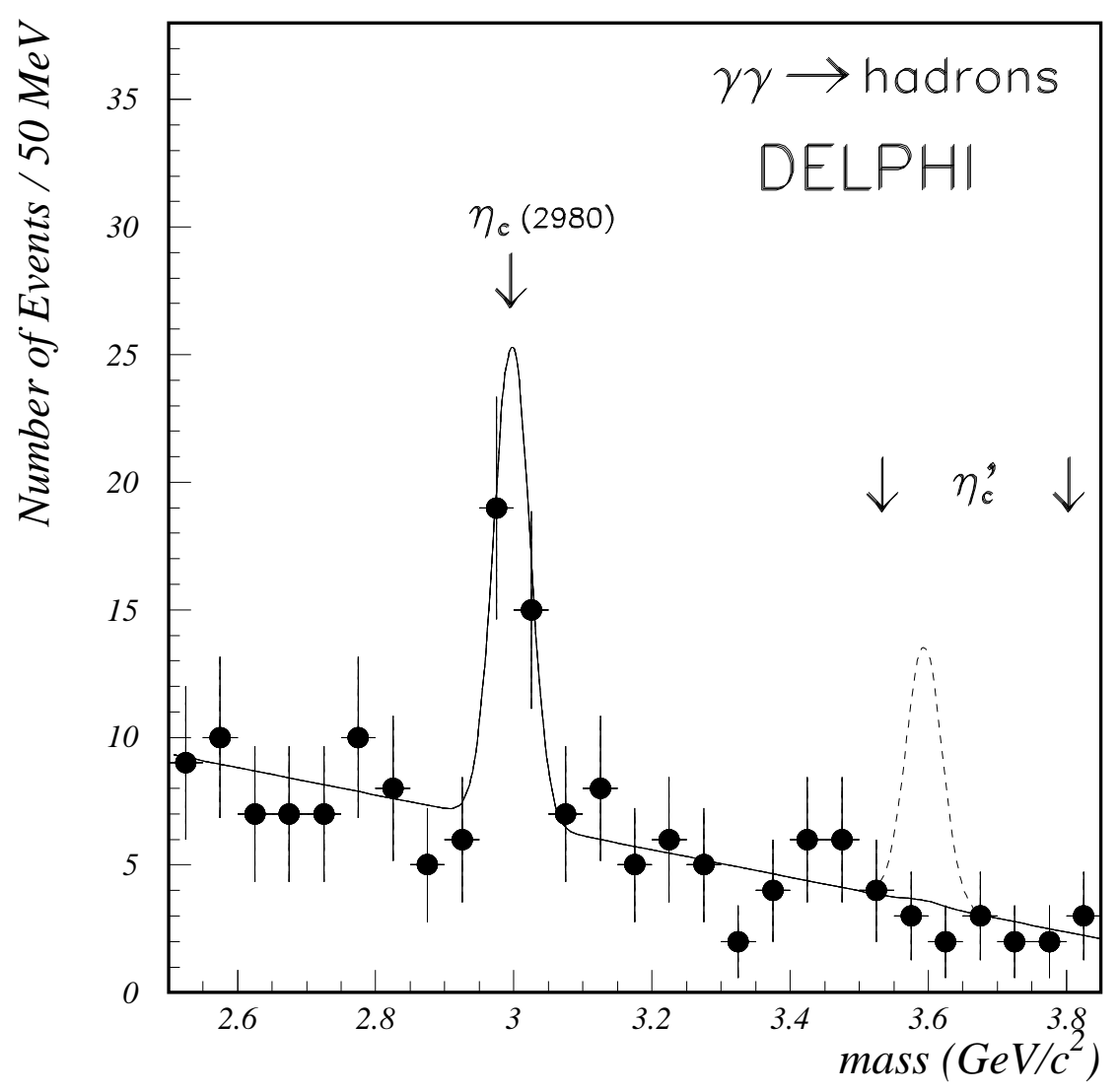

Figure 8.25: The sum of invariant mass distributions from DELPHI for five hadronic channels. The solid curve shows the result of a fit using two Gaussian functions for $\eta_{c}$ and $\eta_{c}^{\prime}$, and a linear background. The dashed curve shows the expected signal for $\eta_{c}^{\prime}$ production, assuming $B\left(\eta_{c}^{\prime} \rightarrow\right.$ hadrons $)=B\left(\eta_{c} \rightarrow\right.$ hadrons $)$ and that $\Gamma\left(\eta_{c}^{\prime} \rightarrow \gamma \gamma\right)=0.75 \Gamma\left(\eta_{c} \rightarrow \gamma \gamma\right)$. This figure is taken from Ref. [98]. 
it is $\left|\psi_{2 S}(0)\right|^{2} /\left|\psi(0)_{1 S}\right|^{2} \times \Gamma\left(\eta_{c} \rightarrow \gamma \gamma\right)=0.6 \times \Gamma\left(\eta_{c} \rightarrow \gamma \gamma\right)$. (See Eq. 8.38) However, DELPHI has obtained a result which suggests that this might not be the case.

In their $\gamma \gamma$ fusion measurements, DELPHI clearly observes $\eta_{c}$ in several hadronic decay channels. In Fig. 8.25 we show the sum of invariant mass distributions from five hadronic channels from DELPHI. The dashed curve in the figure shows the expected signal of $\eta_{c}^{\prime}$ production assuming the prediction of $\Gamma\left(\eta_{c}^{\prime} \rightarrow \gamma \gamma\right) / \Gamma\left(\eta_{c} \rightarrow \gamma \gamma\right)=$ 0.75 by Barnes [99]. A 90\% confidence upper limit was set by DELPHI for the ratio of $\gamma \gamma$ partial widths,

$$
\frac{\Gamma\left(\eta_{c}^{\prime} \rightarrow \gamma \gamma\right)}{\Gamma\left(\eta_{c} \rightarrow \gamma \gamma\right)} \leq 0.34, \quad 90 \% \mathrm{CL} .
$$

Preliminary results from L3 suggest that a much lower limit may be set soon. [90]

\subsection{Summary of Results}

A summary of our results for the resonance parameters of $\eta_{c}$ and $\chi_{2}$, and limits we have set for $\chi_{0}$ and $\eta_{c}^{\prime}$ is presented in Table 8.9.

\subsection{Discussion of Results}

In this section we summarize recently published theoretical predictions for the $\gamma \gamma$ and $g g$ partial widths of the four charmonium states, $\eta_{c}, \eta_{c}^{\prime}, \chi_{0}$ and $\chi_{2}$. The predictions presented in this section are of two varieties - those that attempt a relativistic treatment of the bound state decays to the lowest order in $\alpha_{\mathrm{S}}$, and those which are essentially non-relativistic, but which go to higher orders in $\alpha_{\mathrm{S}}$. Predictions for the 
Table 8.9: Summary of results presented in this dissertation.

\begin{tabular}{|l|l|}
\hline$M\left(\eta_{c}\right)$ & $2982.4_{-2.2}^{+2.3} \mathrm{MeV}$ \\
$\Gamma\left(\eta_{c}\right)$ & $26.9_{-9.5}^{+10.8} \mathrm{MeV}$ \\
$\Gamma\left(\eta_{c} \rightarrow \gamma \gamma\right)$ & $5.7_{-2.3-1.4}^{+2.9+2.9} \mathrm{keV}$ \\
\hline$\Gamma\left(\chi_{2} \rightarrow \gamma \gamma\right)$ & $0.343_{-0.051-0.038}^{+0.053+0.043} \mathrm{keV}$ \\
\hline \multicolumn{2}{|c|}{$90 \%$ confidence upper limits } \\
\hline$\Gamma\left(\chi_{0} \rightarrow \gamma \gamma\right)$ & $1.3 \mathrm{keV}$ \\
\hline$B\left(\eta_{c}^{\prime} \rightarrow \bar{p} p\right) \times B\left(\eta_{c}^{\prime} \rightarrow \gamma \gamma\right)$ & $6 \times 10^{-8}$ \\
for $\Gamma\left(\eta_{c}^{\prime}\right)=10-20 \mathrm{MeV}$ & \\
\hline
\end{tabular}

$\eta_{c}$ and $\eta_{c}^{\prime}$ partial widths are shown in Table 8.10. Predictions for $\chi_{0}$ and $\chi_{2}$ are shown in Table 8.11. 


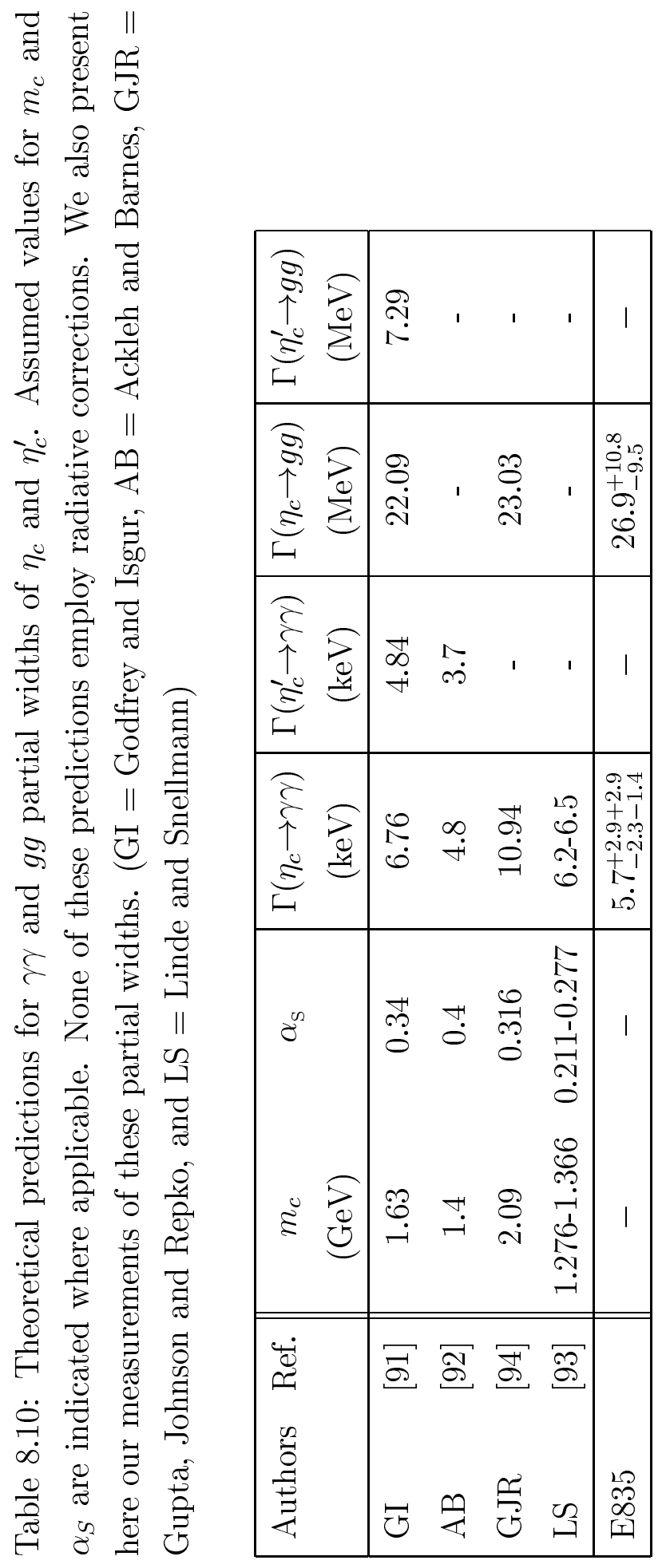




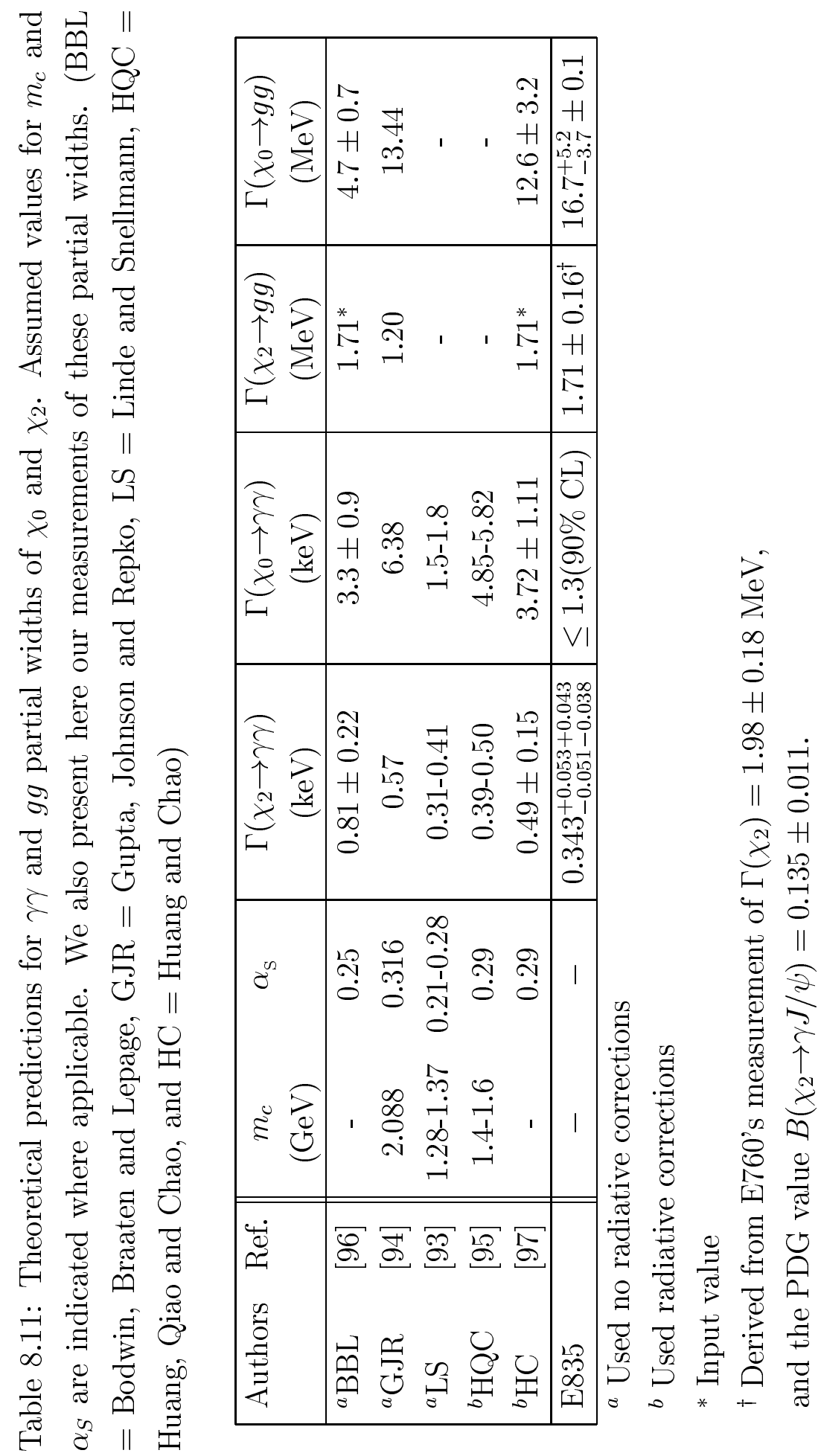




\section{Relativistic calculations}

Several papers in the literature address theoretically the two-gluon and two-photon decays of charmonium states from a relativistic point of view. Much of this work was catalyzed by the first measurements of $\chi_{1}$ and $\chi_{2}$ total widths and branching ratios by $\mathrm{E} 760$.

Two calculations of the relativistic variety are given by Godfrey and Isgur (GI) [91] and Ackleh and Barnes (AB) [92]. GI calculated masses, electromagnetic decay widths, annihilation widths, etc., for "all mesons - from the pion to the upsilon" [91] in a single framework. AB calculated the two-photon decay widths of both light and heavy singlet quarkonia, modeled after a fully-relativistic treatment of singlet positronium.

Both GI and AB use wave functions calculated in a non-relativistic Coulomb+linear potential, and attempt to account for all relativistic corrections to annihilation amplitudes which have been calculated for a free quark-antiquark pair.

In Ref. [93], Linde and Snellmann present a relativistic calculation of $\gamma \gamma$, E1 and M1 decays of charmonium states. Their calculation is done using the Bethe-Salpeter formalism, and a potential with vector one-gluon exchange, and scalar confinement. They determine the parameters of their model by a fit to the charmonium mass spectrum and to the leptonic decay rate of $J / \psi$.

Gupta, Johnson and Repko (GJR) present $\gamma \gamma$ and two-gluon decay rates for charmonium states, [94] using their own "realistic" QCD-inspired potential. [84] GJR claim that they have calculated their transition widths in a fully-relativistic manner, including off-shell quarks. They also claim that their model treats the 
spin-dependent quark-antiquark interaction non-perturbatively. Their model has the rather unusual feature of predicting identical ratios $B\left((c \bar{c})_{R} \rightarrow \gamma \gamma\right) / B\left((c \bar{c})_{R} \rightarrow g g\right)$ for $\chi_{0}, \chi_{2}$ and $\eta_{c}$.

Huang, Qiao and Chao (HQC) present results from relativistic calculations in the Bethe-Salpeter formalism, and they include radiative corrections to NLO in $\alpha_{\mathrm{S}}$. [95] Their potential is a QCD-inspired phenomenological potential, with vector one gluon exchange, and scalar confinement.

\section{Non-Relativistic QCD calculations}

We present two NRQCD predictions which are based on the factorization formalism of Bodwin, Braaten and Lepage (hereafter, BBL) [96].

Early calculations of charmonium decays, including those presented in Ref. [23], attempt to deal with non-perturbative effects by factoring them into the wave function at the origin, $\psi(0)$, in the case of S-state charmonium, or into the derivative of the radial wave function at the origin, $R^{\prime}(0)$, in the case of P-states. BBL noted [96] that this factorization assumption is not valid for P-states, since order $\alpha_{\mathrm{S}}{ }^{3}$ calculations revealed infrared divergences. The presence of those divergences indicated that not all non-perturbative effects could be factored into $R^{\prime}(0)$.

BBL argued for the addition of another phenomenological parameter (aside from $R^{\prime}(0)$ ), which was related to the probability that the $c$ and $\bar{c}$ quarks in the charmonium could be found in a color octet state. By using measured decay rates of $\chi_{2}$ and $\chi_{1}$ to light hadrons, BBL present predictions for the light hadron decays of the other two P-states $\left(\chi_{0}\right.$ and $\left.{ }^{1} P_{1}\right)$, as well as two-photon decays of $\chi_{0}$ and $\chi_{2}$, to lowest 
order in $\alpha_{\mathrm{S}}$. Huang and Chao [97] also use the BBL formalism, with corrections which are next-to-leading order in $\alpha_{\mathrm{S}}$.

All the partial width predictions assume values for the strong coupling constant $\alpha_{\mathrm{S}}\left(m_{c}\right)$ and/or for the charm quark mass $m_{c}$. The assumed values of these parameters are listed in the tables along with the width predictions.

\section{Comparison of E835 results to the theoretical predictions}

The predictions for $\Gamma\left(\eta_{c} \rightarrow \gamma \gamma\right)$ are the other predictions are generally in agreement with our result, $\Gamma\left(\eta_{c} \rightarrow \gamma \gamma\right)=5.7_{-2.3-1.4}^{+2.9+2.9} \mathrm{keV}$. The sole exception is the high value, $10.94 \mathrm{keV}$, predicted by Gupta etal. , [94].

Both the predictions of $\Gamma\left(\eta_{c} \rightarrow g g\right)$ which we presented are surprisingly close to our experimental result of $\Gamma\left(\eta_{c}\right)=26.9_{-8.5}^{+12.1} \mathrm{MeV}$. Interestingly, the prediction by Godfrey and Isgur predates the E760 measurement of $\Gamma\left(\eta_{c}\right)=23.9_{-7.1}^{+12.6} \mathrm{MeV}$ by about ten years.

The predictions for $\Gamma\left(\chi_{2} \rightarrow \gamma \gamma\right)$ are generally in agreement with each other, and with our measured value, $\Gamma\left(\chi_{2} \rightarrow \gamma \gamma\right)=0.343_{-0.051-0.038}^{+0.053+0.043} \mathrm{keV}$. The predictions for $\Gamma\left(\chi_{0} \rightarrow \gamma \gamma\right)$ are more variable, and are generally much larger than the $90 \%$ confidence upper limit, $\Gamma\left(\chi_{0} \rightarrow \gamma \gamma\right) \leq 1.3 \mathrm{keV}$, established by us. 


\section{Chapter 9}

\section{Determination of $\alpha_{\mathrm{S}}$}

The essence of QCD is embodied in its coupling constant, $\alpha_{\mathrm{S}}$. Measurements of $\alpha_{\mathrm{S}}$ at low momentum transfers are extremely important for the understanding of strong interaction physics. At the Lepton-Photon '93 Conference, Frank Wilczek commented that "if you want to be quantitative about $\alpha_{\mathrm{S}}$, then the low energy determinations have a big advantage." [100] In other words, Wilczek was making the point that high $q$ measurements of $\alpha_{\mathrm{S}}$ (those in the vicinity of $q=M\left(Z^{0}\right) \simeq 91.2$ $\mathrm{GeV}$ ) are simply not sensitive to how the strong coupling constant runs, as are

measurements of $\alpha_{\mathrm{S}}$ in the low $q$ region, near the charmonium and bottomonium resonances.

The strong coupling constant varies rather rapidly with $q$ at low energies, and 'slows down' at higher energies. Its variation with $q$ is given by [2] 


$$
\begin{aligned}
\alpha_{\mathrm{S}}(q) & =\frac{4 \pi}{\beta_{0} \ln (\kappa)} \times\left(1-\frac{2 \beta_{1}}{\beta_{0}^{2}} \frac{\ln (\ln (\kappa))}{\ln (\kappa)}\right. \\
& \left.+\frac{4 \beta_{1}^{2}}{\beta_{0}^{4} \ln ^{2}(\kappa)} \times\left[(\ln (\ln (\kappa))-0.5)^{2}+\frac{\beta_{2} \beta_{0}}{8 \beta_{1}^{2}}-1.25\right]\right)
\end{aligned}
$$

where $\beta_{0}=11-(2 / 3) n_{f}, \beta_{1}=51-(19 / 3) n_{f}$, and $\beta_{2}=2857-(5033 / 9) n_{f}+$ $(325 / 57) n_{f}^{2}$, in which $n_{f}$ is the number of quark flavors with masses less than $q . \kappa$ is shorthand for $q^{2} /\left(\Lambda_{\bar{m}}^{n_{f}}\right)^{2}$, and $\Lambda_{\frac{n_{f}}{M S}}$ is the QCD cut-off parameter defined in the $\overline{M S}$ renormalization scheme, for $n_{f}$ flavors. The PDG98 gives the current world

averages of $\Lambda_{\overline{M S}}^{(4)}=305 \pm 25 \pm 50 \mathrm{MeV}$, and $\Lambda_{\overline{M S}}^{(5)}=219_{-23}^{+25} \mathrm{MeV}$. [2] A value of $\alpha_{\mathrm{S}}$ at one scale $\mu_{1}$ may be compared to that at another scale $\mu_{2}$ by taking the ratio of Eq. 9.1 evaluated at $\mu_{1}$ to that evaluated at $\mu_{2}$, using appropriate values of $n_{f}$ and $\Lambda_{\overline{M S}}$ if a flavor threshold or thresholds lie between $\mu_{1}$ and $\mu_{2}$. We use Eq. 9.1 in this way to evolve our measured value of $\alpha_{\mathrm{S}}\left(m_{c}\right)$ up to the mass of $Z^{0}$.

Both $\eta_{c}$ and $\chi_{2}$ annihilate into two gluons. We are therefore able to derive a value of the strong coupling constant $\alpha_{\mathrm{S}}$ at the charm quark mass $m_{c}$, by using both our measurements of the hadronic and two photon partial widths of $\eta_{c}$ and $\chi_{2}$. In order to make this determination, we use the pQCD formulae presented in Sec. 2.5.

\section{$9.1 \alpha_{\mathrm{S}}$ determined by $\eta_{c}$ data}

As we mentioned in Sec. 2.5, $\alpha_{\mathrm{S}}$ may be calculated in the framework of pQCD by taking ratios of the pQCD predictions for gluonic and electromagnetic annihilation widths of charmonium states. This leads to the cancellation of the unknown charm 
quark mass and the radial wave function at the origin, and its derivative at the origin.

There are two ways we can use our results for $\eta_{c}$ to determine $\alpha_{\mathrm{S}}$.

1) If we make the assumption that the wave function at the origin for the $1 \mathrm{~S}$ charmonium states, $J / \psi$ and $\eta_{c}$, is the same, we can calculate $\alpha_{\mathrm{S}}$ by the following:

$$
\frac{\Gamma\left(J / \psi \rightarrow e^{+} e^{-}\right)}{\Gamma\left(\eta_{c} \rightarrow g g\right)}=\frac{8 \alpha_{\mathrm{EM}}^{2}}{3 \alpha_{\mathrm{S}}{ }^{2}} \times \frac{\left(1-5.3 \alpha_{\mathrm{S}} / \pi\right)}{\left(1+4.8 \alpha_{\mathrm{S}} / \pi\right)} .
$$

As we argued in Sec. 8.6, the $g g$ partial width of $\eta_{c}$ is essentially equal to its total width, so we evaluate the above equation by inserting the world average of $\Gamma\left(J / \psi \rightarrow e^{+} e^{-}\right)=5.26 \pm 0.37 \mathrm{keV},[2]$ and our measurement of $\Gamma\left(\eta_{c} \rightarrow g g\right)=\Gamma\left(\eta_{c}\right)=$ $26.9_{-9.5}^{+10.8} \mathrm{MeV}$. Solving Eq. 9.2 for $\alpha_{\mathrm{S}}$, using $\alpha_{\mathrm{EM}}\left(m_{c}\right)=1 / 133.3$ [96], we obtain

$$
\alpha_{\mathrm{S}}\left(m_{c}\right)=0.397_{-0.040}^{+0.030}
$$

where the errors have been obtained by varying the value of $\Gamma\left(\eta_{c}\right)$ within its experimental errors. As seen in Eq. 9.2, the first order radiative correction is nearly a factor of two. This casts serious aspersion on this result.

2) It is also possible to make a comparison between the $\gamma \gamma$ and the $g g$ partial widths of $\eta_{c}$. In doing so, we do not have to make the assumption required above (that the $\eta_{c}$ and $J / \psi$ wave functions at the origin are exactly equal). From Sec. 2.5, the prediction for the ratio of $\gamma \gamma$ to $g g$ partial widths for $\eta_{c}$ is:

$$
\frac{\Gamma\left(\eta_{c} \rightarrow \gamma \gamma\right)}{\Gamma\left(\eta_{c} \rightarrow g g\right)}=B\left(\eta_{c} \rightarrow \gamma \gamma\right)=2.1 \pm 0.4_{-0.5}^{+1.1} \times 10^{-4}=\frac{8 \alpha_{\mathrm{EM}}{ }^{2}}{9 \alpha_{\mathrm{S}}{ }^{2}} \times \frac{\left(1-3.4 \alpha_{\mathrm{S}} / \pi\right)}{\left(1+4.8 \alpha_{\mathrm{S}} / \pi\right)}
$$

Solving the above equation, we find

$$
\alpha_{\mathrm{S}}\left(m_{c}\right)=0.32_{-0.05}^{+0.04}
$$




\section{$9.2 \alpha_{\mathrm{S}}$ as determined by $\chi_{2}$ data}

We can also derive a value of $\alpha_{\mathrm{S}}\left(m_{c}\right)$ using our results for $\chi_{2}$ Like $\eta_{c}, \chi_{2}$ decays via annihilation into two gluons and into two photons. However, $\chi_{2}$ also has a substantial branching fraction for radiative decay, $B\left(\chi_{2} \rightarrow \gamma J / \psi\right)=13.5 \pm 1.1 \%$. [2] The $g g$ width is thus

$$
\begin{aligned}
\Gamma\left(\chi_{2} \rightarrow g g\right) & =\Gamma\left(\chi_{2}\right)-\Gamma\left(\chi_{2} \rightarrow \gamma J / \psi\right)-\Gamma\left(\chi_{2} \rightarrow \gamma \gamma\right) \\
& \simeq 0.865 \pm 0.011 \times \Gamma\left(\chi_{2}\right)
\end{aligned}
$$

using the known values of $\Gamma\left(\chi_{2}\right), B\left(\chi_{2} \rightarrow \gamma J / \psi\right)$ and our measured value of $\Gamma\left(\chi_{2} \rightarrow \gamma \gamma\right)$. Using the above relation for $\Gamma\left(\chi_{2} \rightarrow g g\right)$ in the ratio prediction from pQCD,

$$
\frac{\Gamma\left(\chi_{2} \rightarrow \gamma \gamma\right)}{\Gamma\left(\chi_{2} \rightarrow g g\right)}=\frac{0.343_{-0.051-0.038}^{+0.053+0.043} \mathrm{keV}}{1.71 \pm 0.16 \mathrm{MeV}}=\frac{8 \alpha_{\mathrm{EM}}{ }^{2}}{9 \alpha_{\mathrm{S}}{ }^{2}} \times \frac{\left(1-5.3 \alpha_{\mathrm{S}} / \pi\right)}{\left(1-2.2 \alpha_{\mathrm{S}} / \pi\right)}
$$

Solving this equation, we obtain:

$$
\alpha_{\mathrm{S}}\left(m_{c}\right)=0.361_{-0.020}^{+0.023}
$$

As noted in the previous chapter, Bodwin, Braaten and Lepage [96] (hereafter, BBL) have made a different calculation for the relationship between $\mathrm{P}$ state hadronic widths. Their procedure leads to somewhat different predictions for P-state charmonium decay rate ratios.

In the BBL formalism, the following relationship exists between the hadronic widths (denoted by $\Gamma\left(\chi_{J} \rightarrow L H\right)$ of $\chi_{1}$ and $\chi_{2}$, and the two-photon width of $\chi_{2} \cdot[96]$

$$
\begin{aligned}
\frac{\Gamma\left(\chi_{2} \rightarrow \gamma \gamma\right)}{\Gamma\left(\chi_{2} \rightarrow L H\right)-\Gamma\left(\chi_{1} \rightarrow L H\right)} & =\frac{\Gamma\left(\chi_{2} \rightarrow \gamma \gamma\right)}{(0.865 \pm 0.011) \times \Gamma\left(\chi_{2}\right)-(0.727 \pm 0.016) \times \Gamma\left(\chi_{1}\right)} \\
& =\frac{8 \alpha_{\mathrm{EM}}{ }^{2}}{9 \alpha_{\mathrm{S}}{ }^{2}}
\end{aligned}
$$


Using the measured values of $\Gamma\left(\chi_{2}\right)$ as above and $\Gamma\left(\chi_{1}\right)=0.88 \pm 0.14 \mathrm{MeV}$ from E760 [101], we obtain

$$
\alpha_{\mathrm{S}}\left(m_{c}\right)=0.394_{-0.035}^{+0.043}
$$

Huang and Chao [97] extend the BBL result above to the next order in $\alpha_{\mathrm{S}}$, obtaining

$$
\frac{\Gamma\left(\chi_{2} \rightarrow \gamma \gamma\right)}{\Gamma\left(\chi_{2} \rightarrow L H\right)-\Gamma\left(\chi_{1} \rightarrow L H\right)}=\frac{8{\alpha_{\mathrm{EM}}}^{2}}{9{\alpha_{\mathrm{S}}}^{2}} \times \frac{\left(1-5.33 \alpha_{\mathrm{S}} / \pi\right)}{\left(1-5.17 \alpha_{\mathrm{S}} / \pi\right)}
$$

U This leads to

$$
\alpha_{\mathrm{S}}\left(m_{c}\right)=0.383_{-0.032}^{+0.038}
$$

\subsection{Comparison to Other Results for $\alpha_{\mathrm{S}}$}

As mentioned earlier, there are very few measurements of $\alpha_{\mathrm{S}}(q)$ in the low energy region near $q=m_{c}$. One of them is certainly worth mentioning, because of its proximity. It comes from measurements of the hadronic decays of $\tau$, for which $q=m_{\tau}=1.78 \mathrm{GeV}$. By comparing the semileptonic decay of $\tau,\left(\tau \rightarrow \nu_{\tau}+\right.$ hadrons $)$ to the leptonic decay $\left(\tau \rightarrow \nu_{\tau}+l+\overline{\nu_{l}}\right)$ decay rates, a value of $\alpha_{\mathrm{S}}$ may be obtained. The PDG [2] summarizes the results of these measurements as $\alpha_{\mathrm{S}}\left(m_{\tau}^{2}\right)=0.35 \pm 0.03$, which is consistent with our result.

It is also possible to compare our measurement of $\alpha_{\mathrm{S}}\left(m_{c}\right)$ to the world average of measurements of $\alpha_{\mathrm{S}}$ by extrapolating our measurement to the mass of the $Z$ boson. We can use Eq. 9.1 to scale our result from $q_{0}=m_{c}$ to an equivalent value at the standard $q=M\left(Z^{0}\right)$. 
The average of our $\eta_{c}$ and $\chi_{2}$ results calculated using the prescription of Ref. [23] is $0.354 \pm 0.018$. If we insert $n_{f}=3$ and our result into Eq. 9.1, we find that our measurement of $\alpha_{\mathrm{S}}\left(m_{c}\right)$ corresponds to $\alpha_{\mathrm{S}}\left(M\left(Z^{0}\right)\right)=0.119_{-0.008}^{+0.005} \pm 0.007$, assuming that $m_{c}=1.5 \mathrm{GeV}$. The first error corresponds to our error of 0.018 on $\alpha_{\mathrm{S}}\left(m_{c}\right)$, while the second corresponds to the errors on the PDG averages $\Lambda \frac{(4)}{M S}=305 \pm 25 \pm 50$ $\mathrm{MeV}$, and $\Lambda_{\overline{M S}}^{(5)}=219_{-23}^{+25} \mathrm{MeV}$. Our result is completely consistent with the world average as stated by PDG98 of $\alpha_{\mathrm{S}}\left(M_{Z}\right)=0.119 \pm 0.002$. [2] Our measurement of $\alpha_{\mathrm{S}}\left(m_{c}\right)$

$$
\alpha_{\mathrm{S}}\left(m_{c}\right)=0.354 \pm 0.018
$$

is shown as an open circle in Fig. 9.1 in which we have assumed $m_{c}=1.5 \mathrm{GeV}$. The other experimental measurements of $\alpha_{\mathrm{S}}$ in the figure, and Table 9.1 in which we have listed our result, are taken from a compilation by Bethke. [102]. 
Table 9.1: Measurements of $\alpha_{\mathrm{S}}(Q)$. The first is our result, while the others are taken from the compilation in Ref. [102].

\begin{tabular}{|lll|}
\hline Process & $\mathrm{Q}(\mathrm{GeV})$ & $\alpha_{\mathrm{S}}(Q)$ \\
\hline $\bar{p} p \rightarrow c \bar{c} \rightarrow \gamma \gamma$ & 1.5 & $0.354 \pm 0.018$ \\
\hline DIS & 1.58 & $.375_{-.081}^{+.062}$ \\
DIS & 1.73 & $.295_{-.073}^{+.092}$ \\
$\tau$ decay & 1.78 & $.339_{-0.21}^{+.021}$ \\
DIS & 4.1 & $.223_{-.009}^{+.009}$ \\
DIS & 4.13 & $.220_{-.027}^{+.027}$ \\
DIS & 5.0 & $.215_{-.016}^{+.016}$ \\
DIS & 7.1 & $.180_{-.014}^{+.014}$ \\
$e^{+} e^{-} \rightarrow$ hadrons & 10.52 & $.20_{-.06}^{+.06}$ \\
$\bar{p} p \rightarrow b \bar{b}+X$ & 20.0 & $.145_{-.019}^{+.018}$ \\
$e^{+} e^{-}$event shapes & 22.0 & $.161_{-.011}^{+.016}$ \\
$\bar{p} p \rightarrow \gamma X$ & 24.2 & $.137_{-.014}^{+.017}$ \\
$e^{+} e^{-} \rightarrow$ hadrons & 34.0 & $.146_{-.026}^{+.031}$ \\
$e^{+} e^{-}$event shapes & 35.0 & $.145_{-.007}^{+.012}$ \\
$e^{+} e^{-}$event shapes & 44.0 & $.139_{-.007}^{+.010}$ \\
$e^{+} e^{-}$event shapes & 58.0 & $.132_{-.008}^{+.008}$ \\
$e^{+} e^{-} \rightarrow Z^{0}$ & 91.2 & $.122_{-.005}^{+.005}$ \\
$e^{+} e^{-}$event shapes & 91.2 & $.122_{-.006}^{+.006}$ \\
\hline
\end{tabular}




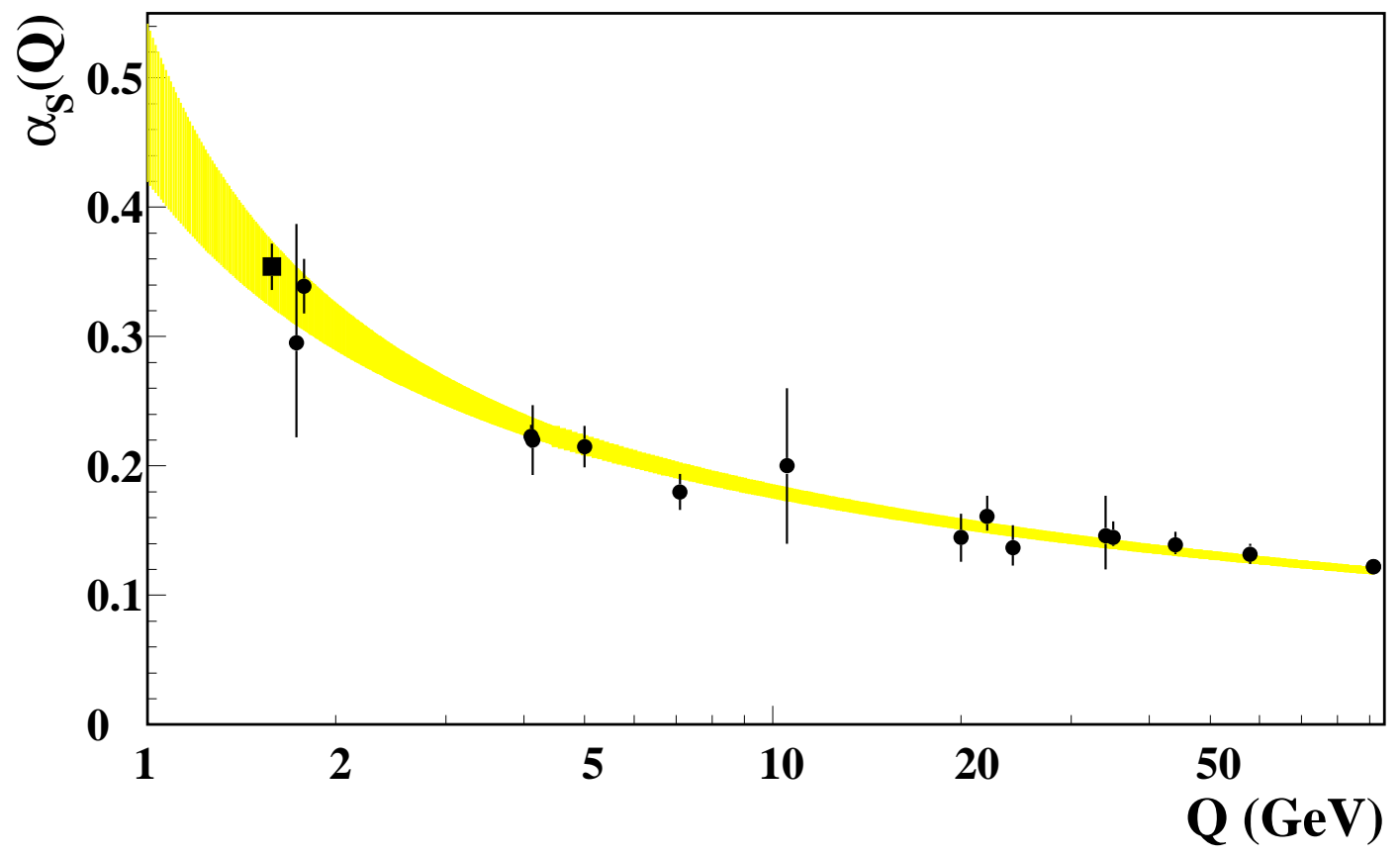

Figure 9.1: Measurements of $\alpha_{\mathrm{S}}(Q)$ from various experiments. [102] The E835 result is indicated by the open circle. The shaded band indicates the evolution of the PDG average value of $\alpha_{\mathrm{S}}\left(m_{Z}\right)=0.119 \pm 0.002$ from $Q=91.2 \mathrm{GeV}$ down to $Q=1 \mathrm{GeV}$, using $\Lambda_{\overline{M S}}^{(4)}=305 \pm 25 \pm 50 \mathrm{MeV}$, and $\Lambda_{\overline{M S}}^{(5)}=219_{-23}^{+25} \mathrm{MeV}$.[2] The thickness of the band is determined by the errors on $\Lambda_{\frac{n_{f}}{M S}}$. 


\section{Chapter 10}

\section{Comments and Reflections}

The results we have presented in this dissertation represent improvements in each case over similar measurements made by our predecessor experiment E760, and those reported by other experiments. The small $\chi_{2} \rightarrow \gamma \gamma$ partial width has been confirmed, and the larger $\eta_{c}$ total width measured by E760 is also supported by our investigation. In the framework of $\mathrm{pQCD}$, we have determined a value of $\alpha_{\mathrm{S}}\left(m_{c}\right)$ which is compatible with other measurements, and has small experimental error.

Our work has also left some troubling unanswered questions. For instance, where is $\eta_{c}^{\prime}$ ? Do we have a chance to see it at all, in $\bar{p} p \rightarrow \eta_{c}^{\prime} \rightarrow \gamma \gamma$ ? Is it anomalously narrow for some reason? Is its coupling to $\bar{p} p$ unexpectedly small? More interestingly, is its

$\gamma \gamma$ decay greatly suppressed, as recent evidence from DELPHI suggests might be the case?

E835', the next run of this succesful family of experiments, is set to run in the fall of 1999. Perhaps this run will finally answer some of these and other questions. 
An investigation of $\chi_{0}$ is planned, the very interesting ratio $\Gamma\left(\chi_{0} \rightarrow \gamma \gamma\right) / \Gamma\left(\chi_{2} \rightarrow \gamma \gamma\right)$ should be measured with good precision. The indications from E835 of a large $\bar{p} p \rightarrow \chi_{0}$ width [78] needs to be confirmed. The identification of the ${ }^{1} P_{1}$ state will be confirmed, and its width and decay modes will be investigated. 


\section{Bibliography}

[1] D. Griffiths, Introduction to Elementary Particles, Wiley Publishers, New York, 1987.

[2] Particle Data Group, European Physical Journal C3 (1998) 1.

[3] M. Gell-Mann, Phys. Lett. 8 (1964) 214.

[4] G. Zweig, CERN report 8182/TH (unpublished) 1964; CERN Report 8419/TH (unpublished) 1964.

[5] M. Gell-Mann Phys. Rev. Lett. 12 (1964) 155.

[6] O. W. Greenberg, Phys. Rev. Lett. 13 (1964) 20.

[7] M. Gell-Mann, Acta Physica Austiraca Suppl. IX (1972) 733

[8] D. Thompson etal., Phys. Rev. Lett. 79 (1997) 1630, G.S. Adams etal., Phys. Rev. Lett. 81 (1998) 5761.

[9] A. Abele etal., Phys. Lett. B423 (1998) 175.

[10] B. J. Bjorken and S. L. Glashow, Phys. Lett. 11 (1964) 255. 
[11] S. L. Glashow, J. Iliopoulos and L. Maiani, Phys. Rev. D2 (1970) 1285.

[12] T. Appelquist and H. D. Politzer, Phys. Rev. Lett. 34 (1975) 43.

[13] E. Eichten, etal. , Phys. Rev. D17 (1980) 3090; ibid. 21 (1980) 313(E); ibid. $21(1980) 203$.

[14] J. L. Richardson, Phys. Lett. 82B (1979) 272.

[15] C. Quigg and J. L. Rosner, Phys. Rep. 56 (1979) 167.

[16] A nice collection of papers relating to the development of QED is found in "Selected Papers on Quantum Electrodynamics", ed. by J. Schwinger, (New York: Dover, 1958).

[17] B. J. Bjorken and S. D. Drell, Relativistic Quantum Mechanics, McGraw-Hill Book Company, New York, 1965, p. (point to Appendix).

[18] J. A. Wheeler, Ann. NY Acad. Sci.48 (1946) 219.

[19] C. N. Yang, Phys. Rev. 77 (1950) 242.

[20] I. B. Khriplovich and A. S. Yelkhovsky, Phys. Lett. B246 (1990) 520.

[21] A. H. Al-Ramadhan and D. W. Gidley, Phys. Rev. Lett. 72 (1994) 1632.

[22] R. Van Royen and V. F. Weisskopf, Nuovo Cim. A50 (1967) 617.

[23] W. Kwong etal., Phys. Rev. D37 (1988) 3210.

[24] J. J. Aubert etal., Phys. Rev. Lett. 33 (1974) 1404. 
[25] J.-E. Augustin et al. , Phys. Rev. Lett. 33 (1974) 1406.

[26] Very unusual proposals for the explanation of the $J / \psi$ observation were offered by prominent physicists such as J. Schwinger, Phys. Rev. Lett. 34 (1974) 37, A. S. and M. Goldhaber, Phys. Rev. Lett. 34 (1974) 36 and H. T. Nieh, T. T. Wu and C. N. Yang, Phys. Rev. Lett. 34 (1974) 49.

[27] The parameters of the Antiproton Accumulator operation may be found in, among others, J. Peoples, IEEE Trans. on Nucl. Sci. NS-30 (1983) 1970, and S. O’Day and F. M. Bieniosek, Nucl. Instr. Meth. A343 (1994) 343. and

[28] A.V. Tollestrup and G. Dugan, "Elementary Stochastic Cooling," in Physics of High Energy Particle Accelerators, AIP Conference Proceedings No. 105, ed. by M. Month, pp. 954-1057.

[29] G. Dugan, CLEO Preprint CLNS-97-1532 (1997).

[30] D. Zholentz etal., Phys. Lett. 96B (1980) 214.

[31] D. Allspach etal., Nucl. Instr. Meth. A410 (1998) 195.

[32] G. Garzoglio, priv. comm., November, 1998.

[33] J. Marques, Ph.D. dissertation, University of California at Irvine (unpublished), 1992.

[34] See, for example, D. Bettoni etal. , IEEE Trans. on Nucl. Sci. 44 (1997) 460, M. Ambrogiani et al. , "Results from the E835 Cylindrical Scintillating-Fiber 
Tracker", Proc. SCI-FI '977 or M. Ambrogiani et al. , "Performance Measurements of Histe-V VLPC Photon Detectors for E835 at FNAL", op. cit.

[35] C. Biino etal. , Nucl. Instr. Meth. A271 (1988) 417.

[36] A. Buzzo etal. , Nucl. Instr. Meth. A391 (1997) 443.

[37] C. Biino etal. , Nucl. Instr. Meth. A317 (1992) 135.

[38] C. M. Ginsburg, Ph.D. dissertation, Northwestern University (unpublished), 1995.

[39] S. Bagnasco et al. , submitted to Nucl. Instr. Meth. A.

[40] K. E. Gollwitzer, Ph.D. dissertation, University of California at Irvine (unpublished), 1993.

[41] S. Trokenheim etal. , Nucl. Instr. Meth. A355 (1995) 308.

[42] T. A. Armstrong etal., Phys. Lett. B385 (1996) 479.

[43] S. Trokenheim, Ph.D. dissertation, Northwestern University (unpublished), 1994.

[44] R. Ray etal., Nucl. Instr. Meth. A307 (1991) 254.

[45] S. Jin and J. L. Rosen, E835 Memo (January 1999), 'E835 Neutral Trigger'.

[46] L. Bartosek etal. , Nucl. Instr. Meth. A301 (1991) 47.

[47] G. Stancari, E835 Internal Memo \#369, 'Measurement of the Magnetic Form Factor of the Proton'. 
[48] J. E. Fast, Ph.D. dissertation, University of California at Irvine (unpublished), 1992.

[49] M. Masuzawa, Ph.D. dissertation, Northwestern University (unpublished), 1994.

[50] T. A. Armstrong etal. ,Phys. Rev. D52 (1995) 4839.

[51] T. A. Armstrong etal., Phys. Rev. D56 (1997) 2509.

[52] M. Ambrogiani, presentation on $\chi_{J}$ angular distributions at Torino E835 Meeting, Feb. 1998. The results of her study should be compared to the E760 paper on the $\chi_{2}$ angular distribution: Phys. Rev. D48 (1993) 3037.

[53] Z. P. Li, F. Close and T. Barnes, Phys. Rev. D43 (1991) 2161.

[54] H. Hamasaki etal. , Phys. Lett. B407 (1997) 185.

[55] M. Artuso et al., Phys. Rev. D50 (1994) 5484.

[56] P. Kroll etal. , Phys. Lett. B316 (1993) 546.

[57] F. James and M. Roos, "MINUIT, Function Minimization and Error Analysis," CERN D506 (Long Writeup).

[58] Particle Data Group, Phys. Rev. D49 (1994) 1.

[59] D. Bisello et al. , Phys. Lett. B350 (1991) 1.

[60] J. Z. Bai etal. , Phys. Rev. Lett. 65 (1990) 1311. 
[61] C. Baglin etal. , Phys. Lett. 187B (1987) 191.

[62] R. M. Balustraitis etal., Phys. Rev. D33 (1986) 629.

[63] J. E. Gaiser etal., Phys. Rev. D34 (1986) 711.

[64] T. M. Himel etal., Phys. Rev. Lett. 45 (1980) 1146.

[65] L3 Collaboration, submission to XXIX Int. Conf. on High Energy Physics, Vancouver, 1998.

[66] V. Savinov and R. Fulton, (CLEO Collaboration) Proc. 10th Int. Workshop on Photon-Photon Collisions, Sheffield, England, 1995, ed. by D. J. Miller et al. , (World Scientific, 1995) p. 203.

[67] H. Albrecht etal., Phys. Lett. B338 (1994) 390.

[68] O. Adriani etal., Phys. Lett. B318 (1993) 575.

[69] W-Y. Chen etal., Phys. Lett. B243 (1990) 169.

[70] H. Aihara etal., Phys. Rev. Lett. 60 (1988) 2355.

[71] T. A. Armstrong etal., Phys. Rev. Lett. 70 (1993) 2988.

[72] Particle Data Group, Phys. Rev. D45 (1992) 1.

[73] J. Dominick etal. , (CLEO Collaboration), Phys. Rev. D50 (1994) 4265.

[74] L3 Collaboration, L3 Preprint \#165

[75] K. Ackerstaff etal. , Phys. Lett. B439 (1998) 197. 
[76] D. A. Bauer etal., Phys. Lett. B302 (1995) 345.

[77] M. Ambrogiani et al., submitted to Phys. Rev. Lett.

[78] T. K. Pedlar, in Proc. Workshop on Heavy Quarks at Fixed Target, Batavia, IL 1998, ed. by J. Butler and H. Cheung, (AIP: New York, 1999), p 369.

[79] R. A. Lee etal., Stanford University Report SLAC-282, (unpublished) 1985.

[80] R. Fulton, Ph.D. dissertation, The Ohio State University (unpublished), 1996.

[81] C. Edwards etal., Phys. Rev. Lett. 48 (1982) 70.

[82] A. Martin and J.-M. Richard, Phys. Lett. 115B (1982) 323.

[83] W. Buchmüller, Y. J. Ng and S.-H. H. Tye, Phys. Rev. D24 (1981) 3003.

[84] S. N. Gupta etal., Phys. Rev. D49 (1994) 1551.

[85] X. Q. Zhu etal., Phys. Rev. D47 (1993) 1155.

[86] E. Eichten and C. Quigg, Phys. Rev. D52 (1995) 1726.

[87] Y.-Q. Chen and R. J. Oakes, Phys. Rev. D53 (1996) 5051.

[88] C. Quigg and J. L. Rosner, Phys. Lett. B71 (1977) 153.

[89] Y.-Q. Chen and Y.-P. Kuang, Phys. Rev. D46 (1992) 1165.

[90] K. K. Seth, priv. comm.

[91] S. Godfrey and N. Isgur, Phys. Rev. D32 (1985) 189. 
[92] E. S. Ackleh and T. Barnes, Phys. Rev. D45 (1992) 232.

[93] J. Linde and H. Snellmann, hep-ph/9703383.

[94] S. N. Gupta, J. M. Johnson and W. W. Repko, Phys. Rev. D54 (1996) 2075.

[95] H-W. Huang, C-F. Qiao and K-T. Chao, Phys. Rev. D54 (1996) 2123.

[96] G. T. Bodwin, E. Braaten and G. P. LePage, Phys. Rev. D46 (1992) R1914, Phys. Rev. D51 (1995) 1125, Erratum: Phys. Rev. D55 (1997) 5853.

[97] H-W. Huang and K-T. Chao, Phys. Rev. D54 (1996) 6850, Erratum: Phys. Rev. D56 (1997) 1821

[98] P. Abreu etal., Phys. Lett. B441 (1998) 479.

[99] T. Barnes, T. E. Browder and S. F. Tuan, Phys. Lett. B385 (1996) 391.

[100] F. Wilczek, in Proc. XVI International Symposium on Lepton and Photon Interactions, Ithaca, NY, 1993, (AIP: New York, 1994), p. 593.

[101] T. A. Armstrong etal., Nucl. Phys. B373 (1992) 35.

[102] S. Bethke, paper presented to the IVth Int. Symp. on Radiative Corrections, Barcelona, 1998, hep-ex/9812026.

[103] M. Jacob and G. C. Wick, Ann. Phys. 7 (1959) 404.

[104] A. D. Martin and T. D. Spearman, Elementary Particle Theory, NorthHolland Publishing Company, Amsterdam, 1970. 
[105] W. Baldini, Ph.D. dissertation, University of Ferrara (unpublished), 1998.

[106] J. J. Zhao, Ph.D. dissertation, Northwestern University (unpublished), 1993.

[107] Sheng Jin, E835 memo (unpublished), Feb. 1999.

[108] G. J. Feldman and R. D. Cousins, Phys. Rev. D57 (.) 3873 


\section{Appendix A}

\section{The CCAL Splitter-Shaper-Discriminator Circuit}

\section{A.1 Introduction}

With the increased instantaneous luminosity which was expected in E835, event rates in all detectors were expected to increase by up to a factor five over typical rates in E760. These event rates would have caused a large increase in the incidence of pileup, that is, the existence of extra clusters in the CCAL due to multiple interactions occurring within the time defined by a single FERA gate. Pileup clusters, if not identified as such, could cause a good event to be discarded.

There are two solutions which we have employed in E835 to reduce the effect of pileup clusters. First, we use shorter FERA ADC gates. In E760, a 150 ns wide FERA gate was used. It would be impossible to reduce the gate width in E835 unless the $\sim 150 \mathrm{~ns}$ wide CCAL pulses could be reduced considerably. This was 
done. Second, in order to identify pileup clusters by timing, TDC's were installed for all 1280 CCAL channels.

Both of these solutions were implemented in E835 by a passive pulse shaping circuit called the Splitter-Shaper-Discriminator Circuit (SSD). The SSD was designed by Jose Marques and FNAL Engineer Claudio Rivetta. In this appendix we describe the testing and performance of the SSD, whose schematic is presented in Fig. A.1, and the setting of final component values for the circuit for each of the four types of CCAL photomultiplier tubes. (three types are indicated in the figure - the 3 " and 2.5" CCAL PMTs use the same circuit components)

\section{A.2 Testing of the SSD Prototype}

After basic design work for the SSD was completed, we received a prototype board containing 16 of the circuits for testing. We were concerned about two aspects of the SSD operation. First, it was essential that its response to input pulses be linear. That is, we wanted the output signal from the SSD have an area which was a linear function of the input pulse area, for a large range of input pulse sizes. Second, we

wanted to make sure that the SSD pulses were narrow enough so that the goals of pileup minimization could be realized.

\section{A.2.1 Linearity of the SSD}

We verified the linearity of the SSD prototype for a large range of input pulse sizes, using a setup represented by the block diagram in Fig. A.2. Light from a nitrogen 

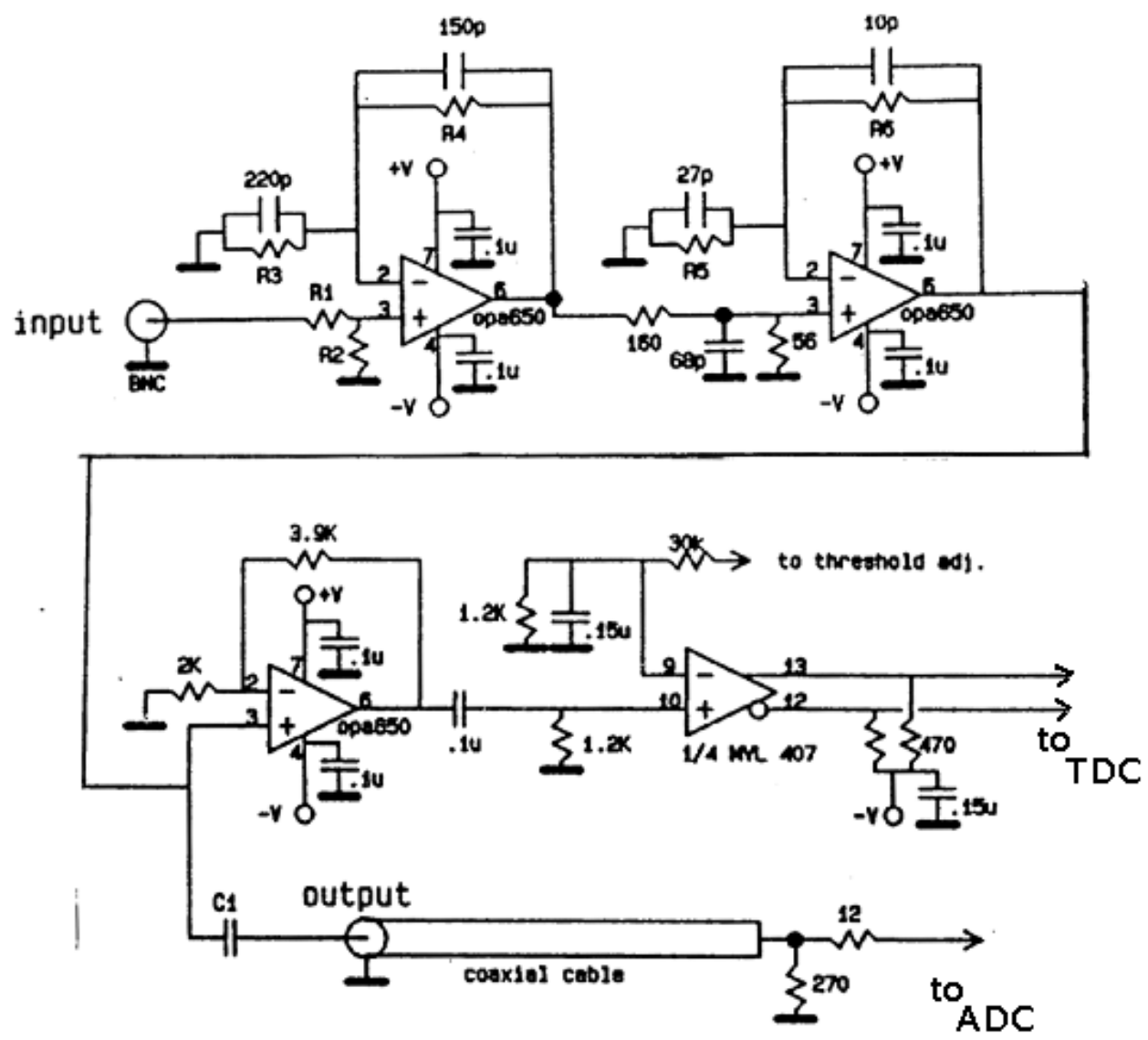

\begin{tabular}{|ccccccccl}
\hline Tube diameter & A1 & R2 & R3 & R4 & R5 & R6 & C1 \\
(3") & 1 & 24 & 27 & $1.2 \mathrm{~K}$ & $1.0 \mathrm{~K}$ & $1.3 \mathrm{~K}$ & $1.3 \mathrm{~K}$ & $22 \mathrm{nF}$ \\
$\left(2^{\prime \prime)}\right.$ & 2 & 30 & 20 & $1.2 \mathrm{~K}$ & 680 & 820 & $1.5 \mathrm{~K}$ & $22 \mathrm{nF}$ \\
$\left(1.5^{\prime \prime}\right)$ & 3 & 30 & 18 & 910 & 680 & 680 & $1.5 \mathrm{~K}$ & $18 \mathrm{nF}$
\end{tabular}

Figure A.1: Schematic diagram of an SSD channel for the shaping of one input signal from a CCAL block. 


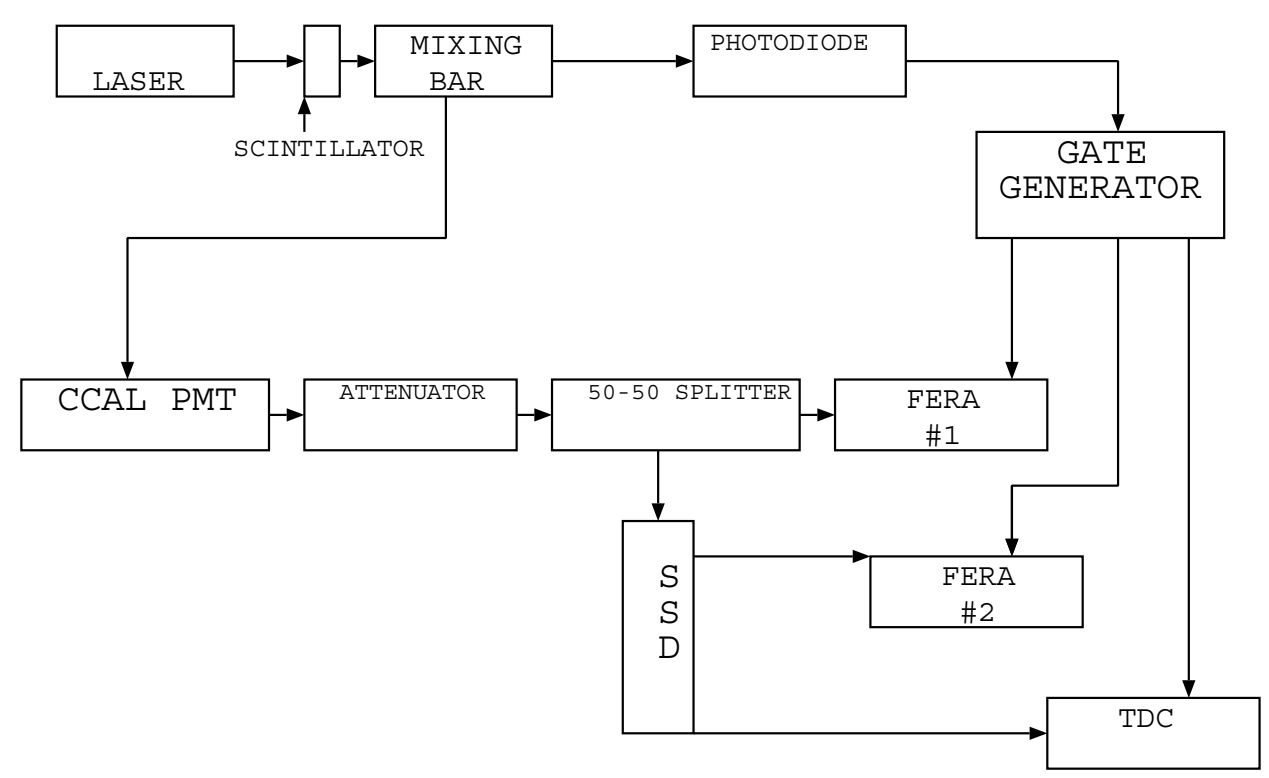

Figure A.2: Test setup for the SSD prototype at FNAL.

laser (Laser Science, Inc. model \#VSL-337ND, peak $\lambda=337 \mathrm{~nm}$ ) was incident upon a scintillator, which produced blue light. The light pulses were transported via a fiber optic cables to the 1280 CCAL blocks. The output signal from a CCAL PMT was passed through a variable attenuator, and then to a resistor junction, which split the signal into two equal parts. One half of the attenuated signal was then input straight into a FERA, while the other was input into the SSD, and the SSD output was passed into a second FERA.

The linearity of each of the 16 channels of the prototype SSD board was checked by comparing the FERA ADC outputs of the shaped and unshaped signals. This was done for a large range of pulse sizes (approximately 3 to 900 ADC counts). The pulse sizes were changed by changing the attenuator. The ratio of outputs for the shaped to the unshaped pulses for one channel of the prototype SSD is shown in 
Fig. A.3. As is apparent from the fit to the data presented in the figure, the circuit is linear to a very good approximation.

\section{A.2.2 Shaped Pulses and Pileup}

In E760, the unshaped CCAL pulse at the input of the FERA had a very long tail, or undershoot, caused by distortion of the signal due to its passage through $\sim 300 \mathrm{~ns}$

of delay cable. With such a pulse shape, an untriggered interaction occurring before a real event trigger resulted in what appeared to be 'extra' clusters, which could possibly be confused as due to the triggered event. In the higher rate environment of E835, the background due to these phenomena would have been significantly worse if no improvement to the CCAL signal readout were made. The SSD was used to alleviate this problem in two ways.

First, it shapes pulses such that the tail of the pulse returns to baseline more quickly, and therefore the effect of each untriggered interaction is shorter. We found that the SSD output pulses for even very large $(\sim 1000$ ADC counts $)$ pulses generally returned to baseline in less than 100 ns. This reflects marked improvement over the unshaped pulses, which remain below baseline for several $\mu$ sec.

Second, the SSD output pulses have an overshoot rather than an undershoot. The overshoot is not integrated by the FERAs, so 'pileup' clusters should not occur at all.

Since the input signal shapes for laser pulses are different than those from 'real' events, the shaped pulses also have different shapes. (See Fig. A.4) In particular, the overshoot for a laser pulse is significantly larger than that for cosmic rays (and 
$96 / 05 / 02 \quad 12.20$
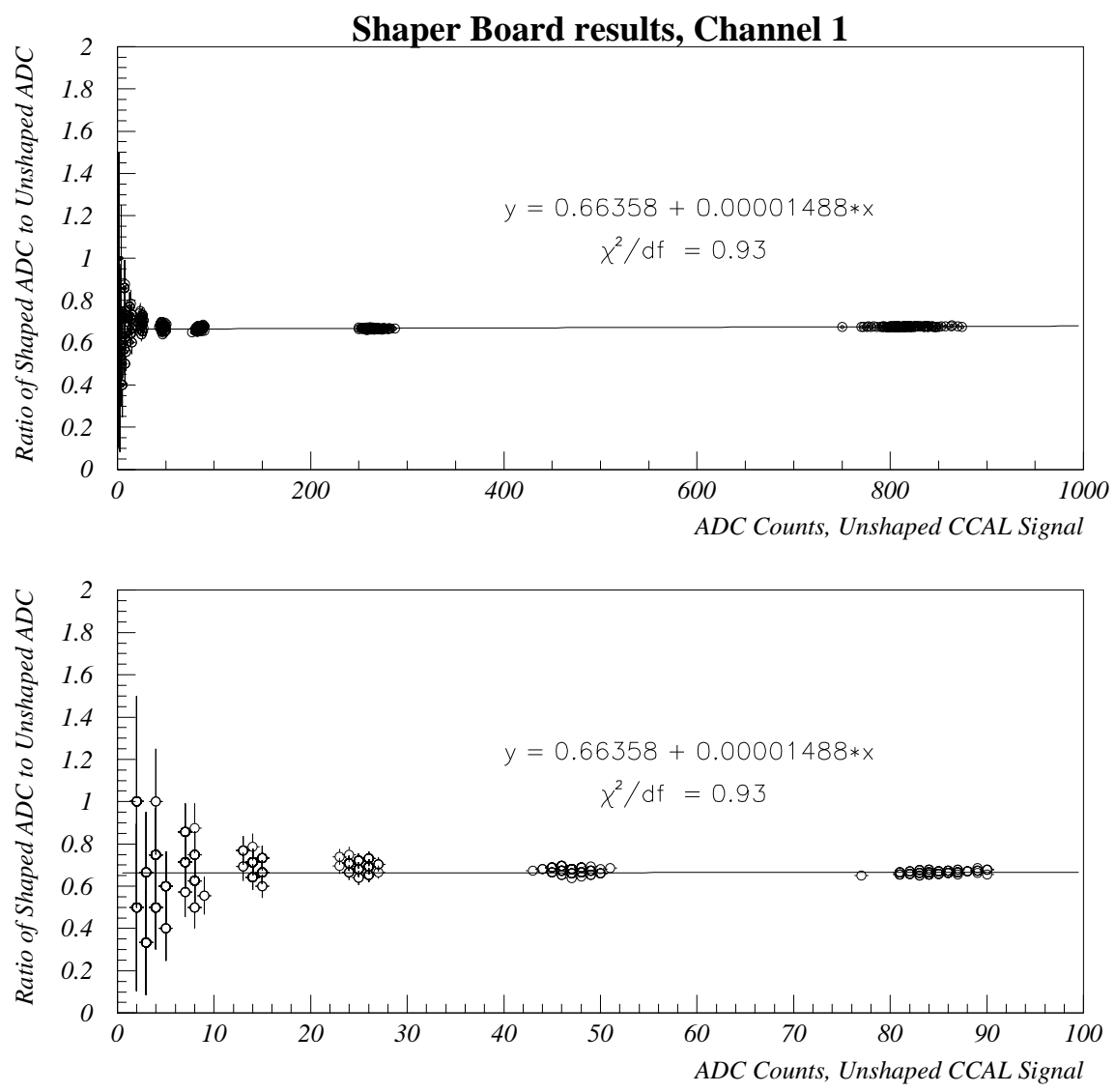

Figure A.3: Result of linearity test for Channel 1 of the SSD prototype. 
for real events, as we found at BNL). As we can see from the figure, the cosmic ray pulse returns substantially to baseline in $\lesssim 80 \mathrm{~ns}$.

\section{A.3 Timing of Shaped Pulses}

In E760 precise timing information was unavailable for signals from individual CCAL elements. The lack of such information in the higher rate environment of E835 would have been a great problem, so the SSD was designed to provide timing for individual signals as well. This is accomplished in the SSD (see Fig. A.1) by splitting off part of the shaped signal and passing it through a discriminator, whose threshold is adjustable. The discriminated signal from each of the 16 channels on an SSD circuit board is then passed to a corresponding TDC.

\section{A.4 Beam Test at Brookhaven}

We took the SSD prototype to the B2 test beam line at the Brookhaven National Laboratory in order to study the SSD prototype performance using 'real' events, and to finalize the components on the SSD for production. The beam line delivered an unseparated beam of $3 \mathrm{GeV}$ negatively charged particles; we triggered on electrons by means of the test setup shown in Fig. A.5. Two pairs of crossed scintillator paddles identified the passage of charged particles through a threshold Cerenkov detector, which identified electrons. The coincidence of the five signals (one from each scintillator paddle, and one from the Čerenkov detector) was used to define 

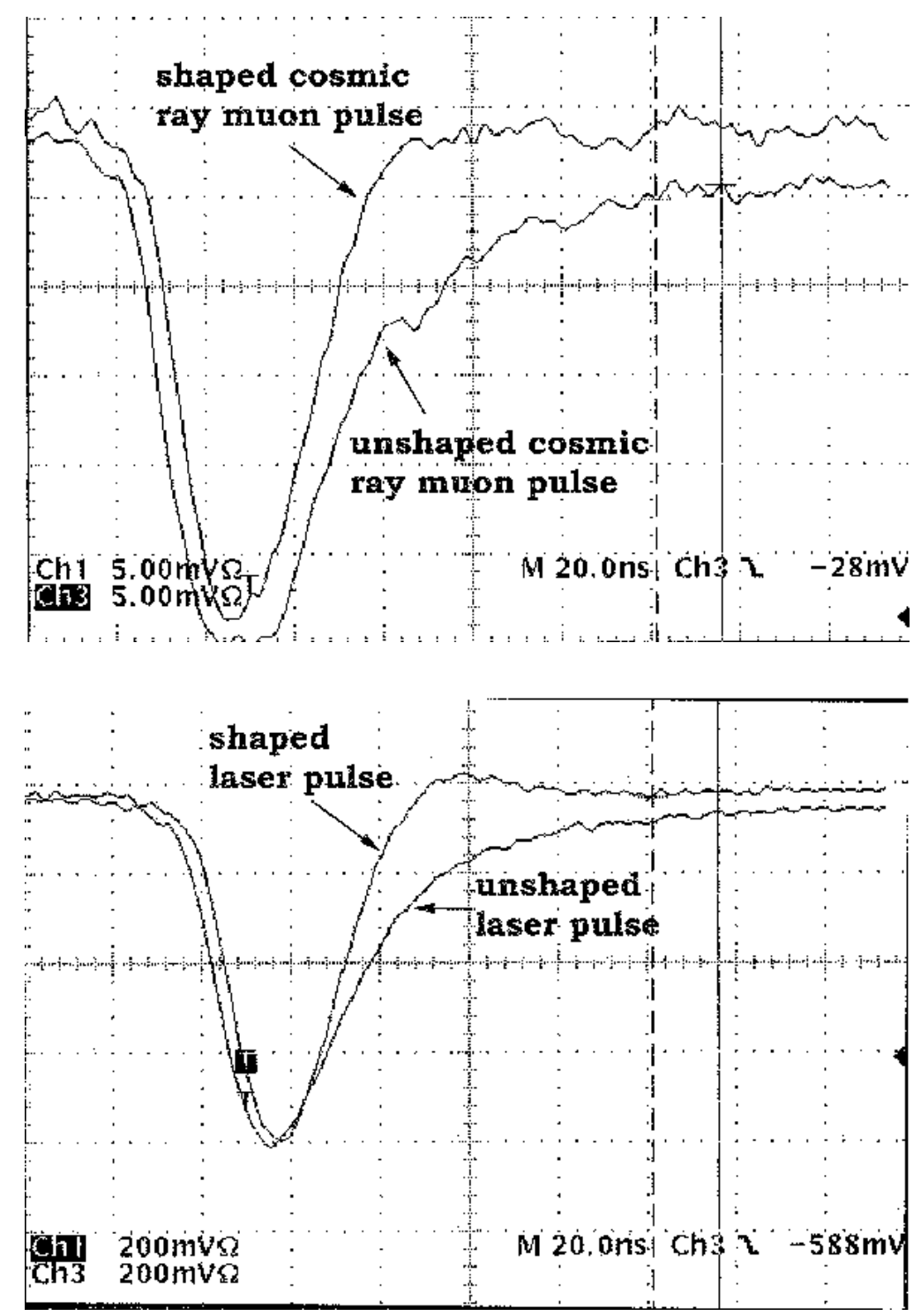

Figure A.4: Comparison of the CCAL signal pulses due to (top) a cosmic ray muon and (bottom) a pulse from the laser system. As noted in Chapter 4, the shape of the two signals is similar enough to enable use of the laser data as a CCAL gain monitor. 


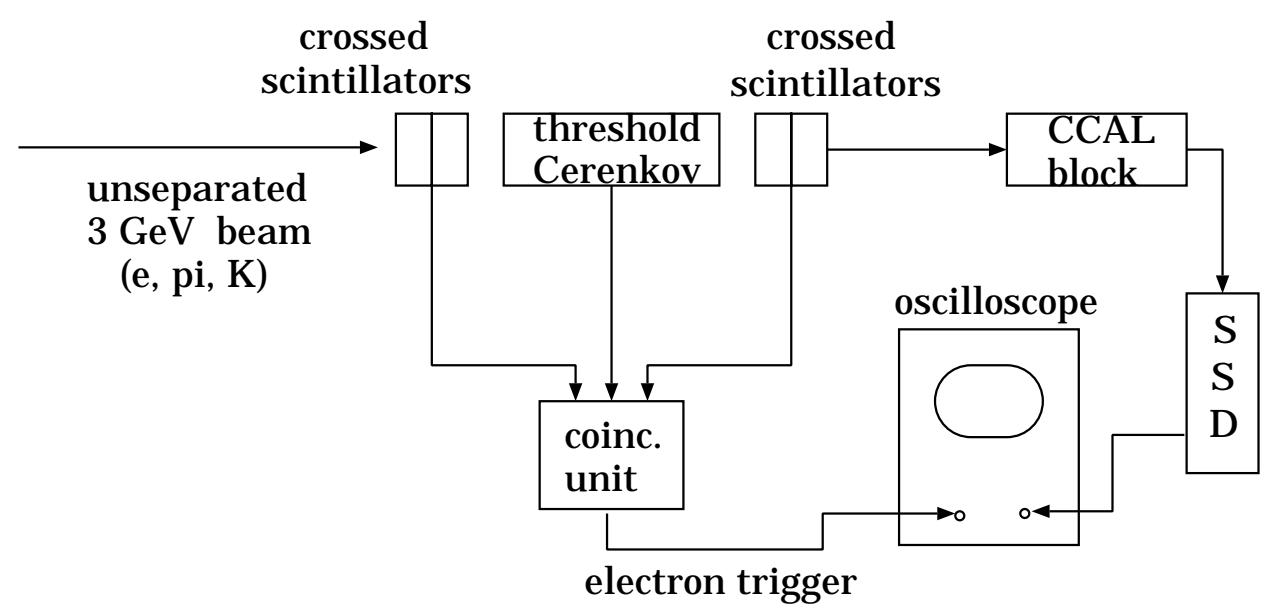

Figure A.5: Setup at Brookhaven for the beam test of the SSD prototype.

the trigger for a digital oscilloscope, to which the CCAL PMT signal was passed. During the test, we optimized the SSD circuit components for each of the PMT sizes $\left(3^{\prime \prime}, 2.5^{\prime \prime}, 2^{\prime \prime}\right.$ and $\left.1.5^{\prime \prime}\right)$ which we use in the CCAL. By optimized, we mean the following:

1. The output pulse should be as narrow as practical, i.e. the pulse should cross the baseline as quickly as possible. Narrower pulses allow the use of narrower FERA gates, which is to be preferred.

2. The pulse should have a small overshoot, rather than an undershoot, as was the case in E760. Undershoots can give rise to extra clusters which must be removed by offline analysis, as was mentioned above.

3. The pulse should cross the baseline (and more specifically, the TDC threshold) smoothly; otherwise, the discriminator on the SSD may be tripped falsely, giving rise to false TDC hits. 
Table A.1: Results for BNL test of the SSD

\begin{tabular}{|lrr|}
\hline Ring (PMT size) & Typical Full Pulse Width & Overshoot \% \\
\hline $1-16(3 " \& 2.5 ")$ & $\sim 70 \mathrm{~ns}$ & $2.1 \%$ \\
$17-18(2 ")$ & $\sim 50 \mathrm{~ns}$ & $1.4 \%$ \\
$19-20(1.5 ")$ & $\sim 45 \mathrm{~ns}$ & $1.5 \%$ \\
\hline
\end{tabular}

4. The overshoot should return to baseline as soon as possible.

We set the final component values for three different SSD circuits; the shape of signals from the $3^{\prime \prime}$ and $2.5^{\prime \prime}$ tubes are similar enough that we required only three different circuits rather than four. The final pulse shape is governed by the RC time constants of the two filters in the circuit (the first two elements after the input - see Fig. A.1) and the circuit's overall time constant, which may be varied by changing the output capacitor. During the test, we adjusted these component values "on the fly", as we took data. The original component values had been suggested by using Spice (an electronics simulation package for the PC), and we made adjustments to these values in order to achieve the best pulse shapes for each PMT using the 3 $\mathrm{GeV}$ electron pulses. Final component values are shown in the table at the bottom of Fig. A.1. 


\section{Appendix B}

\section{Performance of the Central Calorimeter (CCAL)}

There are several criteria by which the performance of the CCAL may be evaluated. These include energy resolution for individual clusters, angular resolutions in both

$\theta$ and $\phi$, and the variation of CCAL gain (or calibration) constants, which relate the amount of charge collected by the CCAL PMTs to the energy deposited in the attached CCAL blocks.

The energy and angular resolutions are in fact as much measures of the performance of the clusterizing algorithms discussed in Sec. 6.1 as of the intrinsic character of the detector. These resolutions are best determined by studying the characteristics of two body decays. Since two-photon decays $\bar{p} p \rightarrow \gamma \gamma$ are neither prolific in their yield, nor easy to identify, these resolutions are determined using a clean sample of $J / \psi \rightarrow e^{+} e^{-}$events. As was emphasized in Chapter 3, while these resolutions are important in identifying the decay products, they are not of primary importance in determining the precision of our measurements of charmonium state masses and 


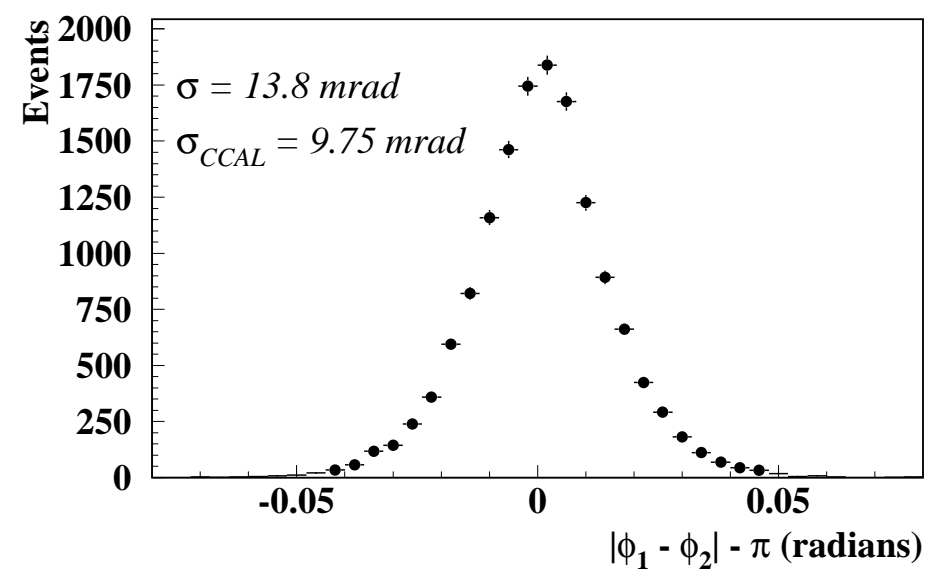

Figure B.1: Azimuthal angle $(\phi)$ resolution for $J / \psi \rightarrow e^{+} e^{-}$events.

widths precisely.

The gain constant variation is a reflection of the physical changes in the lead glass of the CCAL due to radiation damage or PMT aging. It is monitored by two methods - first, by an iterative procedure with $\pi^{0}$ mass reconstruction, using $\pi^{0} \pi^{0}$ events collected at each energy, and second, by means of the data from the laser monitor which was described in Sec. 5.6.

\section{B.1 Angular Resolutions}

We are interested in both the azimuthal angle $(\phi)$ resolution and the polar angle $(\theta)$ resolution. The azimuthal angle resolution is rather simple to determine. We discuss it first. 


\section{Azimuthal angle $(\phi)$ Resolution}

The resolution for the azimuthal angle $\phi$ may be determined by examining the deviation from coplanarity of the reconstructed angles $\phi_{1}$ and $\phi_{2}$ of the electrons in the $J / \psi \rightarrow e^{+} e^{-}$event sample. For these events we can construct a plot of the variable $\left|\phi_{1}-\phi_{2}\right|-\pi$, which should be a Gaussian function distributed about zero. Fig. B.1 shows the $\phi$ resolution determined in this manner, and the result of a Gaussian fit to the distribution. The $\sigma_{\phi}$ determined from the fit is simply related to the CCAL resolution for $\phi$ by $\left(\sigma\left(\left|\phi_{1}-\phi_{2}\right|-\pi\right)\right)^{2}=\left(\sigma\left(\phi_{1}\right)\right)^{2}+\left(\sigma\left(\phi_{2}\right)\right)^{2}=2(\sigma(\phi))^{2}$, since the resolution should not depend on $\phi$. Thus the fit result of $\sigma_{\phi}=13.8 \mathrm{mrad}$

corresponds to a single cluster resolution of $\sigma_{\phi}^{C C A L}=13.8 / \sqrt{2}=9.75 \mathrm{mrad}$.

\section{Polar angle $(\theta)$ Resolution}

In E760 the CCAL polar angle resolution was determined by comparing angles measured by the CCAL to those measured by the charged tracking detectors, whose resolution was significantly better than that of the CCAL. [40] In E835, the scintillating fiber $(\mathrm{SF})$ detector replaces the tracking detectors for $\theta$ measurements for the charged particles. For the purposes of this dissertation we are interested in determining the polar angle resolution of the CCAL. This can be done in two ways: measure the overall $\theta$ resolution of CCAL by measuring $\theta$ of both particles from a two-body decay with the CCAL, or measure $\theta$ for one particle in the CCAL and the other in the SF, if that would lead to better results. To make the choice it is necessary to determine the $\theta$ resolution of both SF and CCAL. We calculate in this section the resolution for $\theta$ of the SF, and compare it to that of the CCAL. 
For a two-body process such as $\bar{p} p \rightarrow J / \psi \rightarrow e^{+} e^{-}$, there is a simple kinematical relation between the laboratory polar angles $\theta_{1}$ and $\theta_{2}$ of the two electrons. We can use the measurement of the angle $\theta_{1}$ to predict the angle $\theta_{2}$ :

$$
\theta_{2}^{\text {pred }}=\sin ^{-1}\left(\frac{s \sin \theta_{1}^{\text {meas }}}{E_{T O T}^{2}+p_{\bar{p}}^{2}-2 E_{T O T} p_{\bar{p}} \cos \theta_{1}^{\text {meas }}}\right) .
$$

The distributions for $f\left(\theta_{1}^{\text {meas }}, \theta_{2}^{\text {meas }}\right) \equiv\left(\theta_{2}^{\text {meas }}-\theta_{2}^{\text {pred }}\right)$ for four different regions of $\theta_{1}^{\text {meas }}$ are shown in Fig. B.2, with both electrons detected in either the SF or in the CCAL. It is clear from the figures that the "effective $\theta$ resolution" * for the SF is actually poorer than that of the CCAL. This result may appear counter-intuitive, but arises primarily from the finite extension of the interaction region.

The following schematic calculation makes this easy to understand. Figure B.3 illustrates the geometry of particle detection. Let the interaction region be spread uniformly, $\pm a$ about the central point. Let a particle exiting the central target point at an angle $\theta$ be detected at a point on the detector. Let the intrinsic uncertainty in this point be $\pm b$ on the detector (SF or CCAL). It can be shown that the extreme angles $\theta_{1}$ and $\theta_{2}$ are:

$$
\begin{aligned}
& \theta_{1}=\tan ^{-1}\left(\frac{r \sin \theta-b \cos \theta}{r \cos \theta+a+b \sin \theta}\right) \\
& \theta_{2}=\tan ^{-1}\left(\frac{r \sin \theta+b \cos \theta}{r \cos \theta-a-b \sin \theta}\right) .
\end{aligned}
$$

Let us consider a mid-range polar angle, $\theta=37.2^{\circ}$. In this case, for $\mathrm{SF}, r \simeq 24.3$ $\mathrm{cm}$, and for CCAL, $r=131.54 \mathrm{~cm}$. Let us assume reasonable estimates for $b$. For

*as distinguished from the nominal resolution, which depends only on the $z$ position of the detected particle in the SF or CCAL detector 

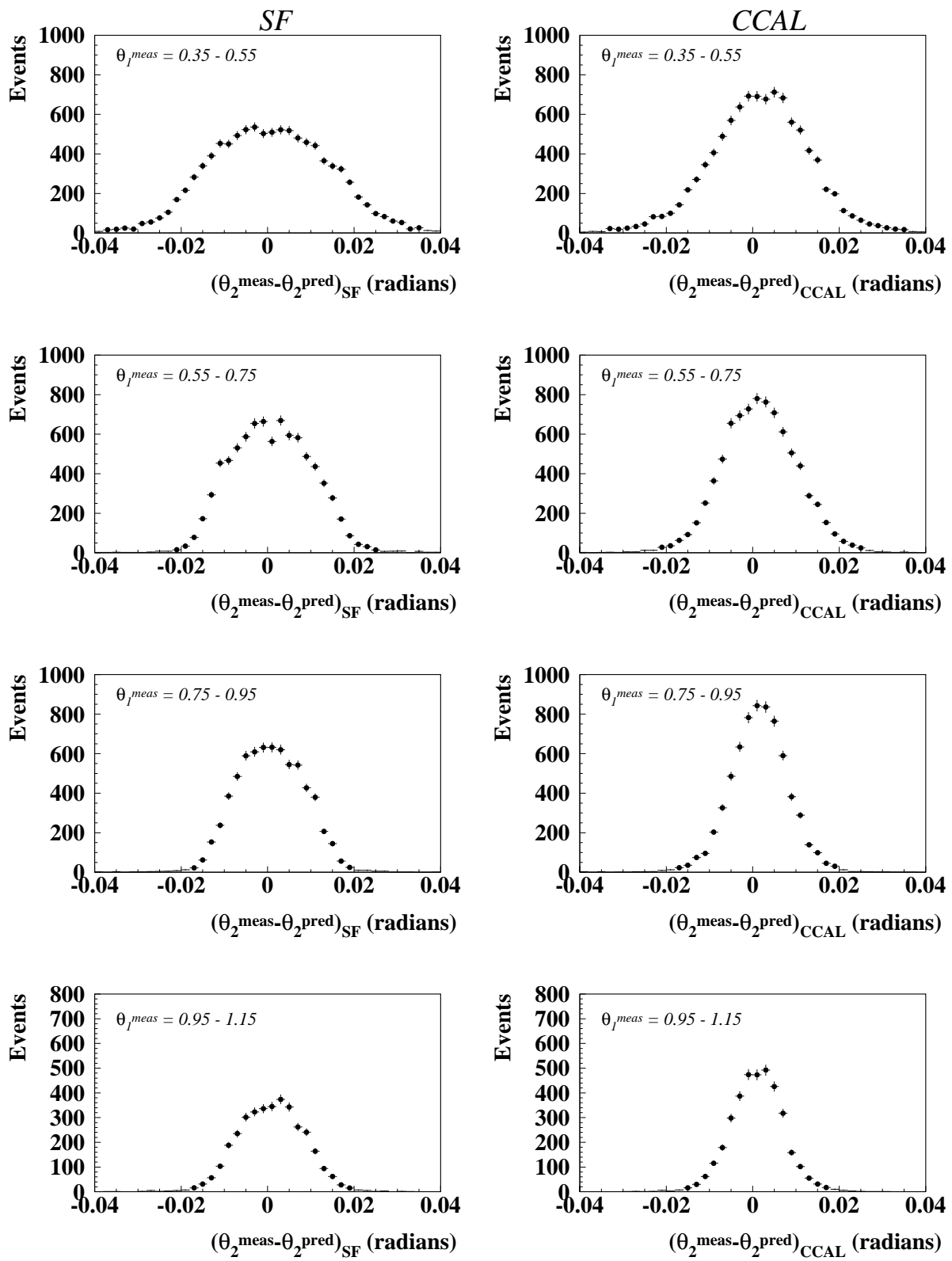

Figure B.2: Distributions of $\theta_{2}^{\text {meas }}-\theta_{2}^{\text {pred }}$ for intervals of $\theta_{1}$ as measured by the SF (left) and by the CCAL (right). 


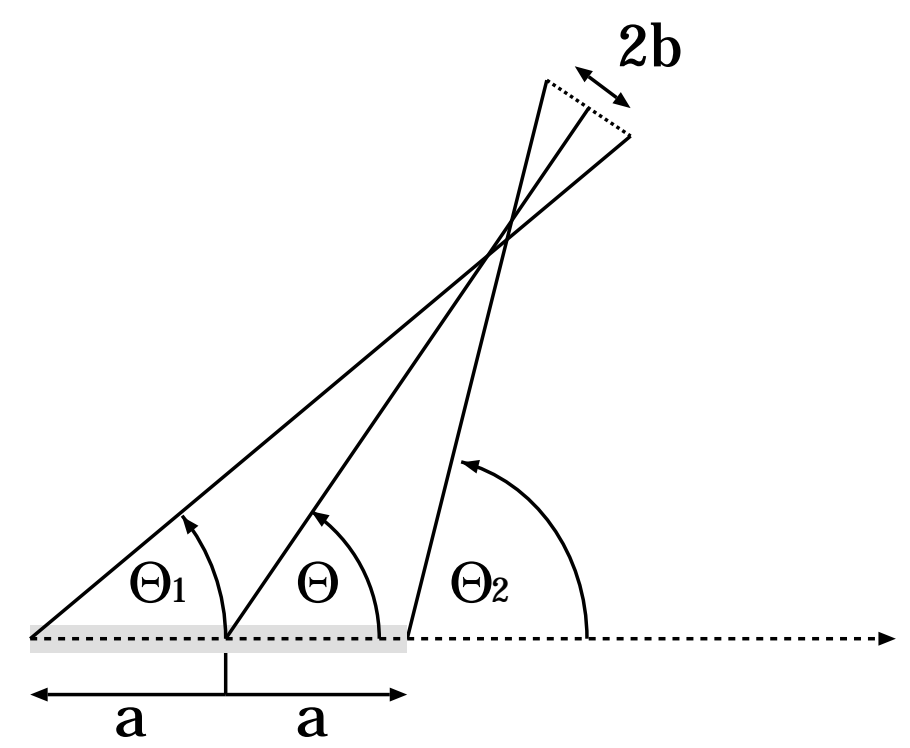

Figure B.3: Schematic showing the geometry of particle detection. The interaction region, of full length $2 a$ is indicated by the dots. The uncertainty in the position detection, at the detector, is $\pm b$.

CCAL, $b=5 \mathrm{~mm}$, and for SF, $b=0.17 \mathrm{~mm}$. With these values, we find the results shown in Table. B.1.

Table B.1 shows that despite the SF's superior position resolution, when the finite target extension is taken into account, the SF's "effective angular resolution" becomes a factor 2 worse than that of CCAL. This is because the SF is nearer to the

Table B.1: Results of schematic calculation of the effect of finite target region and intrinsic position resolution on the "effective" CCAL and SF resolution for $\theta$.

\begin{tabular}{|l|l|l|}
\hline & with $2 a=0 \mathrm{~mm}$ & with $2 a=10 \mathrm{~mm}$ \\
\hline$\theta(\mathrm{CCAL})$ & $37.2^{\circ} \pm 0.22^{\circ}$ & $37.2^{\circ} \pm 0.35^{\circ}$ \\
$\theta(\mathrm{SF})$ & $37.2^{\circ} \pm 0.04^{\circ}$ & $37.2^{\circ} \pm 0.74^{\circ}$ \\
\hline
\end{tabular}


interaction region by more than a factor five. This conclusion is borne out by a more realistic Monte Carlo calculation considering a more realistic Gaussian distribution for the uncertainty in position resolution for both SF and CCAL.

In view of the above, we have decided to use the measurements of both $\theta_{1}$ and $\theta_{2}$ in CCAL in order to estimate the $\theta$ resolution of CCAL.

The resolution $\sigma_{\theta}^{C C A L}$ is not given simply by the standard deviation of the distributions shown in Fig. B.2. The error on $f=\theta_{2}^{\text {meas }}-\theta_{2}^{\text {pred }}$ is given by:

$$
\sigma_{f}^{2}=\sigma^{2}\left(\theta_{2}^{\text {meas }}\right)+\sigma^{2}\left(\theta_{2}^{\text {pred }}\right)
$$

We obtain $\sigma\left(\theta_{2}^{\text {pred }}\right)$ by differentiating Eq. B.1:

$$
\sigma\left(\theta_{2}^{\text {pred }}\right)=\sigma\left(\theta_{1}^{\text {meas }}\right) \times\left[\frac{\left(\frac{A \cos \theta_{1}^{\text {meas }}-B}{A-B \cos \theta_{1}^{\text {meas }}}\right)}{\sqrt{\left(A^{2}-1\right)+\left(B^{2}+1\right) \cos ^{2} \theta_{1}^{\text {meas }}-2 A B \cos \theta_{1}^{\text {meas }}}}\right]
$$

where $A=\left(E_{T O T}^{2}+p_{\bar{p}}^{2}\right) / s$ and $B=2 E_{T O T} p_{\bar{p}} / s$.

We can write Eq. B.4 as $\sigma\left(\theta_{2}^{\text {pred }}\right)=g\left(\theta_{1}^{\text {meas }}\right) \sigma\left(\theta_{1}^{\text {meas }}\right)$, where $g\left(\theta_{1}^{\text {meas }}\right)$ is the expression in the square brackets. Thus,

$$
\sigma_{f}^{2}=\sigma^{2}\left(\theta_{2}^{\text {meas }}\right)+g\left(\theta_{1}^{\text {meas }}\right)^{2} \sigma^{2}\left(\theta_{1}^{\text {meas }}\right)
$$

In Fig. B.4 we have plotted $\sigma_{f}$ as a function of $\theta_{2}$. The $\sigma_{f}$ were obtained by fitting the individual distributions of $f=\theta_{2}^{\text {meas }}-\theta_{2}^{\text {pred }}$ for small intervals of $\theta_{1}^{\text {meas }}$. (as illustrated in Fig. B.2 for larger intervals of $\theta_{1}^{\text {meas }}$ ) Then, we fitted the $\sigma_{f}$ as a function of $\theta_{1}^{\text {meas }}$, and found that the observed shape could be accomodated by errors $\sigma\left(\theta_{1,2}^{\text {meas }}\right)$ which were linear functions of $\theta_{1,2}^{\text {meas }}: \sigma\left(\theta_{1,2}^{\text {meas }}\right)=\alpha+\beta \times \theta_{1,2}^{\text {meas }}$. The result of this fit to the $\sigma_{f}$ is shown in Fig. B.4. 


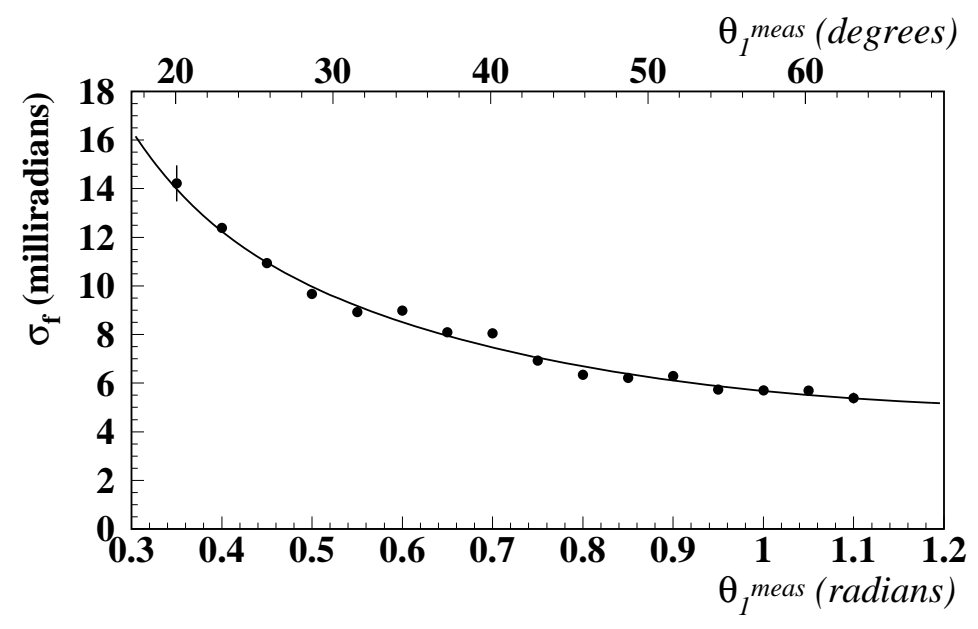

Figure B.4: The distribution of the standard deviation $\sigma_{f}$ of $f=\theta_{2}^{\text {meas }}-\theta_{2}^{\text {pred }}$ as a function of $\theta$ for electrons from $\bar{p} p \rightarrow J / \psi \rightarrow e^{+} e^{-}$events. The curve is the result of a fit to the $\sigma_{f}$ assuming that $\sigma\left(\theta_{1,2}^{\text {meas }}\right)=\alpha+\beta \theta_{1,2}^{\text {meas }}$.

We may also wish to estimate the $\sigma\left(\theta_{1,2}^{\text {meas }}\right)$ by the schematic calculation method we used previously. In Table B.2 we show the results, assuming reasonable position errors $b=2 \mathrm{~mm}$ at $\theta=20^{\circ}$ and $b=7 \mathrm{~mm}$ at $\theta=65^{\circ}$.

Finally, in Fig. B.5 we have plotted the results for $\sigma\left(\theta_{1,2}^{\text {meas }}\right)$ obtained by the fit to $\sigma_{f}$ and by the schematic method. The real errors $\sigma\left(\theta_{1,2}^{\text {meas }}\right)$ probably lie somewhere between the two lines.

Table B.2: Results of the schematic calculation of "effective" CCAL resolutions for $\theta$ at $20^{\circ}$ and $65^{\circ}$, using $a=5 \mathrm{~mm}$. Here we have assumed a varying CCAL position error $b$.

\begin{tabular}{|l|ll|l|}
\hline$\theta_{\text {nominal }}$ & $\mathrm{b}$ & $\mathrm{r}$ & $\theta$ \\
\hline $20^{\circ}$ & $2 \mathrm{~mm}$ & $196.3 \mathrm{~cm}$ & $20^{\circ} \pm 0.11^{\circ}$ \\
$65^{\circ}$ & $7 \mathrm{~mm}$ & $89.44 \mathrm{~cm}$ & $65^{\circ} \pm 0.74^{\circ}$ \\
\hline
\end{tabular}




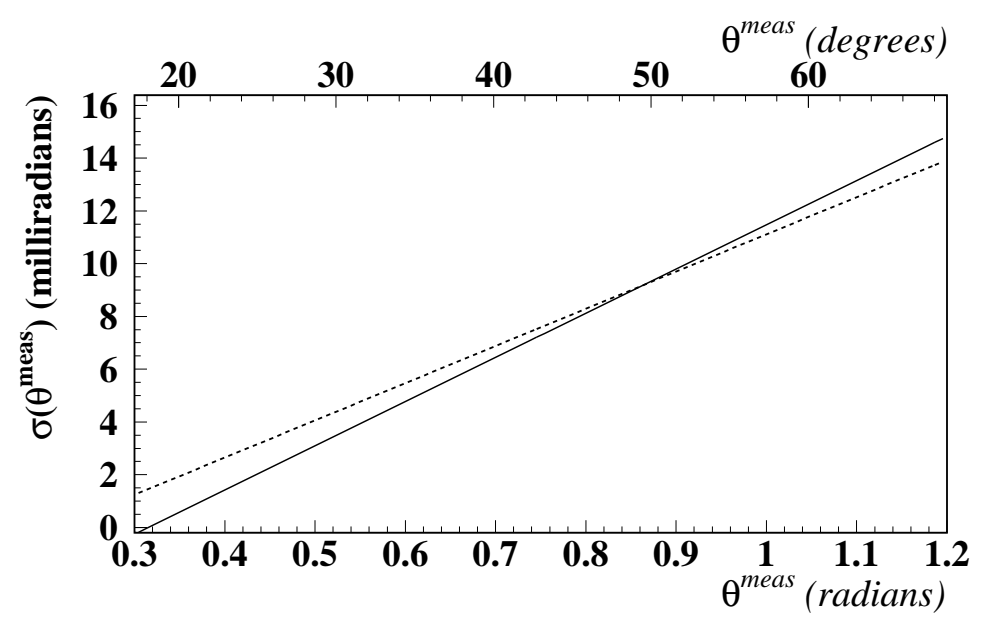

Figure B.5: The results of two estimates of the dependence of the errors $\sigma\left(\theta^{\text {meas }}\right)$ on $\theta^{\text {meas }}$. The solid line is the result of a fit to the measured $\sigma_{f}$ assuming that $\sigma\left(\theta_{1,2}^{\text {meas }}\right)=\alpha+\beta \theta_{1,2}^{\text {meas }}$. The dashed line is a result of using the schematic method, assuming a position error $b=2 \mathrm{~mm}$ at $\theta=20^{\circ}$ and $b=7 \mathrm{~mm}$ at $\theta=65^{\circ}$.

\section{B.2 Energy Resolution}

The average energy resolution of the CCAL for large energy clusters can similarly be examined using $J / \psi \rightarrow e^{+} e^{-}$events. The predicted energy of an electron in a $J / \psi \rightarrow e^{+} e^{-}$decay is given by

$$
E_{e^{-}}^{\text {pred }}=\frac{s}{2\left(E_{T O T}-p_{\bar{p}} \cos \theta_{e^{-}}^{\text {meas }}\right)} .
$$

Differentiating this with respect to $\theta_{e^{-}}$, we obtain

$$
\sigma\left(E_{e^{-}}^{\text {pred }}\right)=\frac{\left(s \times p_{\bar{p}} / 2 E_{\text {TOT }}^{2}\right) \sin \theta_{e^{-}}^{\text {meas }}}{\left(1-\left(p_{\bar{p}} / E_{\text {TOT }}\right) \cos \theta_{e^{-}}^{\text {meas }}\right)^{2}} \sigma\left(\theta_{e^{\text {meas }}}\right) \equiv \mathcal{F}\left(\theta_{e^{-}}\right) \sigma\left(\theta_{e^{-}}^{\text {meas }}\right)
$$

and therefore, the error on the quantity $\Delta E=E_{e^{-}}^{\text {meas }}-E_{e^{-}}^{\text {pred }}$ is given by

$$
\sigma^{2}(\Delta E)=\sigma_{E^{\text {meas }}}^{2}+\sigma_{E^{\text {pred }}}^{2}=\sigma_{E^{\text {meas }}}^{2}+\mathcal{F}^{2}\left(\theta_{e^{-}}^{\text {meas }}\right) \sigma^{2}\left(\theta_{e^{-}}^{\text {meas }}\right)
$$



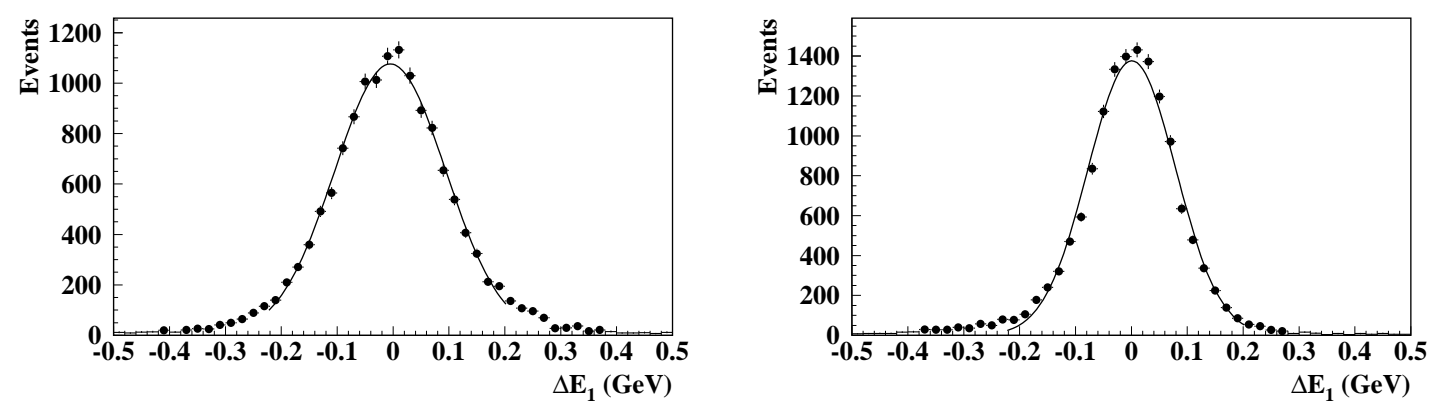

Figure B.6: Average energy resolution for electrons from $J / \psi \rightarrow e^{+} e^{-}$events with average energy (left) $E \simeq 2 \mathrm{GeV}$ and (right) $E \simeq 3.1 \mathrm{GeV}$. The curves are the result of fits to the data. For each, $\sigma_{E} \approx 5.6 \% \times \sqrt{E}$.

Over the range of angles covered by the CCAL, $\sim 20^{\circ}$ to $\sim 65^{\circ}$, the expected energies are $4.2 \mathrm{GeV}$ to $1.3 \mathrm{GeV}$. Given the resolution for $\theta^{\text {meas }}$ in Table B.2, and the result of Eq. B.7, the error $\sigma\left(E^{\text {pred }}\right)$ ranges from $\sim 8$ to $\sim 22 \mathrm{MeV}$. This error can be neglected in comparison to $\sigma\left(E^{\text {meas }}\right)$, which was found in E760 to be $\sim 6 \% \times \sqrt{E^{\text {meas }}} \simeq 70-110 \mathrm{MeV}$. (see, e.g., Ref. [101]) Roughly, the error $\sigma(\Delta E)$ can be expected to be equal to $\sigma\left(E^{\text {meas }}\right)$ for these large energy clusters. The distributions of $\Delta_{E}$ for $J / \psi \rightarrow e^{+} e^{-}$events for average electron energies $\langle E\rangle=2$ $\mathrm{GeV}$ and $\langle E\rangle=3.1 \mathrm{GeV}$ is shown in Fig. B.6. We have fit each to a Gaussian function, and each fit results in a standard deviation $\sigma_{E^{\text {meas }}}$ of $\sim 5.6 \% \times \sqrt{E}$. This is consistent with the average energy resolution found in E760.

More important for our studies is the energy resolution for low energy photons. Our greatest concern is that low energy photons from asymmetric $\pi^{0} \rightarrow \gamma \gamma$ decays are detected with sufficient energy resolution so that the two photons may be reconstructed as a $\pi^{0}$. We can check that this is the case by examining the distribution 


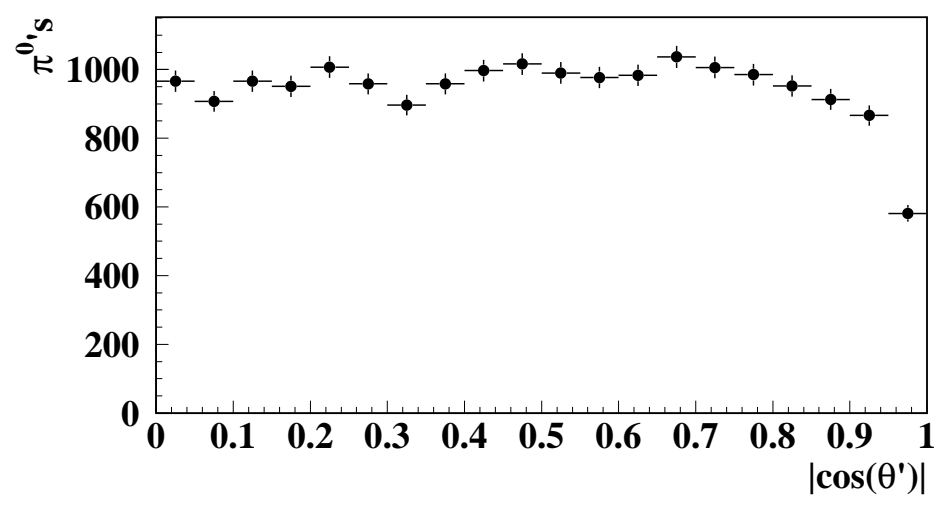

Figure B.7: Number of reconstructed $\pi^{0}$ 's as a function of $\left|\cos \theta^{\prime}\right|$, where $\theta^{\prime}$ is the angle of one of the photons in the $\pi^{0}$ reference frame. The dip at $\left|\cos \theta^{\prime}\right| \simeq 1$ indicates that asymmetric decays are being lost.

of reconstructed $\pi^{0}$ events as a function of the angle $\theta^{\prime}$ of one of the photons in the $\pi^{0}$ reference frame, measured relative to the $\pi^{0}$ direction. This angle is expressed simply as $\left(\cos \theta^{\prime}\right)=\left(E_{1}-E_{2}\right) /\left(\beta_{\pi^{0}}\left(E_{1}+E_{2}\right)\right)$, where $\beta_{\pi^{0}}$ is the speed of the $\pi^{0}$ in the center of mass frame. Since $\pi^{0}$ 's decay isotropically, we expect that this distribution is flat in $\cos \theta^{\prime}$. If the measured distribution shows a deviation from isotropy near $\left|\cos \theta^{\prime}\right|=1$, it indicates a problem of reconstructing asymmetric $\pi^{0}$ decays. If a deviation from isotropy is seen near $\left|\cos \theta^{\prime}\right|=0$, then a problem of reconstructing symmetric decays is indicated.

The $\cos \theta^{\prime}$ distribution for $\pi^{0} \pi^{0}$ events collected at $\sqrt{s}=2990 \mathrm{MeV}$ is shown in Fig. B.7. There is no noticeable drop-off in the distribution near $\left|\cos \theta^{\prime}\right|=0$, and we can therefore conclude that the symmetric decays are being reconstructed correctly. There is a drop, however, in the distribution near $\left|\cos \theta^{\prime}\right|=1$. We believe that this 
drop-off is due to the asymmetric $\pi^{0}$ decays in which a photon is lost. It therefore provides an indication of the level of feed-down background to expect from $\pi^{0} \pi^{0}$ and $\pi^{0} \gamma$ mimicking $\gamma \gamma$

\section{B.3 CCAL Gain Variation}

The gain constant (or calibration constant) for a CCAL block is the coefficient G in the relation $E_{i}=G_{i} \times A_{i}$, where $E_{i}$ is the energy deposited in the block by an incident electron or photon, and $A_{i}$ is the charge collected by the PMT, measured in ADC counts by the FERA. Constant, perhaps, is not the proper term, since as time passes, this constant changes, either due to PMT aging or radiation damage of the lead glass.

Gross monitoring of the CCAL gains was provided in E835 by a laser system which pulsed each block at a constant rate throughout the run. Fine monitoring and calibration was performed by examination of $\pi^{0} \pi^{0}$ events on a stack-by-stack basis, as was done in E760. We describe each of these techniques briefly here.

\section{B.3.1 Laser Monitoring}

Gross variations of the CCAL gains were monitored by use of data from the laser monitor trigger (See Sec. 5.6). For each data run, there were $\sim 1000$ laser triggers recorded, by which the average response of each CCAL channel could be determined. This system provided a relative run-by-run diagnostic on each CCAL channel, though it was good only for large variations $(\gtrsim 5 \%)$, limited primarily 
by statistics. Unfortunately, while the laser was capable of pulse rates up to 200 times faster than we used $(0.1 \mathrm{~Hz})$ the scintillator which produced visible blue light from the laser's incident UV pulses suffered degradation of light output at rates higher than about $1 \mathrm{~Hz}$. Variation of light output from the laser/scintillator system was not a concern in and of itself, since the pulses were also observed by a pair of photodiodes for normalization purposes. However, the loss in absolute intensity at high pulse rates was too rapid and too large for a higher-statistics sample to be obtained for each run. The intent behind the laser system, though, was only to provide a monitor for gross-scale variations in the CCAL gain constants, which it did satisfactorily.

\section{B.3.2 Gain Constant Calculation}

The prolific $\bar{p} p \rightarrow \pi^{0} \pi^{0}$ reaction offers us a nice means of calibrating the CCAL, though it requires a somewhat tedious iterative procedure. Most of this work, described in an E835 collaboration memo, [107], was done by our colleague Sheng Jin, using the procedure described in Ref. [106].

The method begins by selecting a clean sample of $\pi^{0} \pi^{0}$ events from the stack for which gain constants are required. The measurement of the angles $\theta$ and $\phi$ of one $\pi^{0}$ in the event are taken as a given, and then using two-body kinematics, the other $\pi^{0}$ 's energy and angles are predicted. A $\chi^{2}$ function is calculated,

$$
\chi^{2}=\sum_{i=1} n \frac{\sum_{j=1} m\left(g_{j} A_{i j}-E_{i}\right)^{2}}{\sigma_{i}},
$$

where the sum with index $j$ is performed over the number of blocks in the two $\gamma$ 


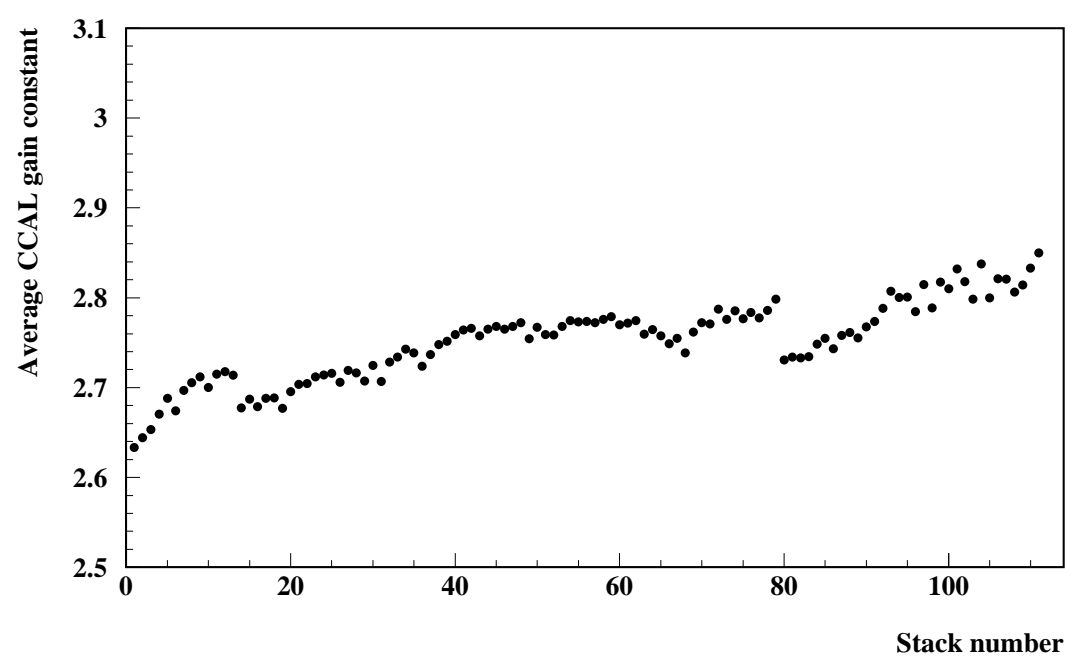

Figure B.8: Variation of the average CCAL channel gain constant, from the beginning of the E835 run (Stack 1) to the end (Stack 110). The general upward trend of the gain constants indicates the darkening the CCAL lead glass blocks due to accumulated radiation dosage during the run.

clusters for a given $\pi^{0}$, and the sum with index $i$ is over all $\pi^{0}$ in the $\pi^{0} \pi^{0}$ events. $g_{j}$ is the gain constant for the $j$ th block, $A_{i j}$ is the number of ADC counts for the $j$ th block of the $i$ th $\pi^{0}$, and $\sigma_{i}$ is the energy error estimated from the formula $\sigma_{i}=6 \% / \sqrt{E(G e V)}+1.4 \%$. This $\chi^{2}$ function is then minimized with respect to the $g_{j}$, and then the newly-calculated gain constants are used to recalculate energies $E_{i}$, and the procedure iterated until the $g_{j}$ converge. Throughout, the masses of the $\pi^{0}$ are constrained to $M\left(\pi^{0}\right)=139.57 \mathrm{MeV}$.

Over the course of the E835 run, 110 stacks were used to calculate gain constants. The variation of the average CCAL channel gain constant is plotted in Fig. B.8 in order to show the change due to radiation damage and/or PMT aging. The general 
trend of the constants is upward, consistent with the hypothesis that accumulated radiation dosage darkens the lead glass (i.e. decreases the light collected by the CCAL PMTs per unit energy deposited in a block). The discrete drop of $\sim 1 \%$ at stack 13 corresponds to a long shutdown in December 1996, during which it is possible that the lead glass cured slightly. The larger drop $(\sim 2 \%)$ at stack 80 is not understood. 


\section{Appendix $\mathrm{C}$}

\section{Angular Distribution of the Reactions $\bar{p} p \rightarrow \eta_{c} \rightarrow \gamma \gamma$ and $\bar{p} p \rightarrow \chi_{2} \rightarrow \gamma \gamma$}

In order to evaluate the efficiency of a cut on the variable $\cos \left(\theta^{*}\right)$ for the processes $\bar{p} p \rightarrow \eta_{c} \rightarrow \gamma \gamma$ and $\bar{p} p \rightarrow \chi_{2} \rightarrow \gamma \gamma$, we need to know the angular distribution for the reactions.

The angular distribution for $\eta_{c}$, since it is a pseudoscalar state, is necessarily isotropic. The efficiency of the cut $\left|\cos \left(\theta^{*}\right)\right| \leq 0.2$ for $\eta_{c} \rightarrow \gamma \gamma$ region is therefore 0.2 .

The $\bar{p} p \rightarrow \chi_{2} \rightarrow \gamma \gamma$ process does not have an isotropic angular distribution, however, but it can be calculated following the formalism of Jacob and Wick. [103]. The organization of this section follows a similar discussion in Ref. [48]

\section{$\underline{\bar{p} p \rightarrow \chi_{2}}$}

The two-particle helicity state describing the initial $\bar{p}$ and $p$ can be written as a single ket, $\left|\theta, \phi ; \lambda_{1}, \lambda_{2}\right\rangle$, where $\theta$ and $\phi$ are the angles defining the direction of the $\bar{p}$ 


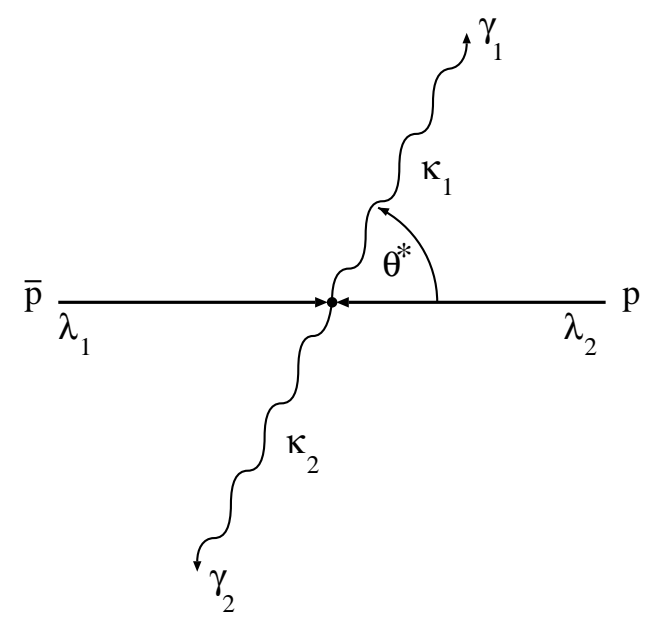

Figure C.1: Schematic of the reaction $\bar{p} p \rightarrow \chi_{2} \rightarrow \gamma \gamma . \lambda_{1}$ and $\lambda_{2}$ are the helicities of the $\bar{p}$ and $p$, respectively, while $\kappa_{1}$ and $\kappa_{2}$ are the helicities of the outgoing photons. $\theta^{*}$ is the angle in the CM frame between the incoming $\bar{p}$ momentum and one of the photons.

in the CM frame, and $\lambda_{1}$ and $\lambda_{2}$ are the helicities of the $\bar{p}$ and $p$, respectively. We can write this state more conveniently by expanding over a complete set of angular momentum states, a là Jacob and Wick, [103]:

$$
\left|\theta, \phi ; \lambda_{1}, \lambda_{2}\right\rangle=\sum_{J, M, \lambda_{1}^{\prime}, \lambda_{2}^{\prime}}\left|J^{\prime}, M^{\prime} ; \lambda_{1}^{\prime}, \lambda_{2}^{\prime}\right\rangle\left\langle J^{\prime}, M^{\prime} ; \lambda_{1}^{\prime}, \lambda_{2}^{\prime} \mid \theta, \phi ; \lambda_{1}, \lambda_{2}\right\rangle .
$$

We note that the second term is given by

$$
\left\langle J^{\prime}, M^{\prime} ; \lambda_{1}^{\prime}, \lambda_{2}^{\prime} \mid \theta, \phi ; \lambda_{1}, \lambda_{2}\right\rangle=\left(\frac{2 J^{\prime}+1}{4 \pi}\right)^{1 / 2} \delta_{\lambda_{1} \lambda_{1}^{\prime}} \delta_{\lambda_{2} \lambda_{2}^{\prime}} \mathcal{D}_{M^{\prime}, \lambda}^{J^{\prime}}(\phi, \theta,-\phi),
$$

where $\lambda=\lambda_{1}-\lambda_{2}$. Then, inserting this into Eq. C.1, we have

$$
\left|\theta, \phi ; \lambda_{1}, \lambda_{2}\right\rangle=\sum_{J^{\prime}, M^{\prime}}\left(\frac{2 J^{\prime}+1}{4 \pi}\right)^{1 / 2} \mathcal{D}_{M^{\prime}, \lambda}^{J^{\prime}}(\phi, \theta,-\phi),\left|J^{\prime}, M^{\prime} ; \lambda_{1}, \lambda_{2}\right\rangle
$$

Similarly, we can write the angular momentum state of the $\chi_{2}$ as $|J, M\rangle$. We note at this point that when calculating the amplitude for $\bar{p} p \rightarrow \chi_{2}$, the sum over $J^{\prime}$ and 
$M^{\prime}$ becomes equal to the single term with $J^{\prime}=J=2$ and $M^{\prime}=M$, by angular momentum conservation. Then the amplitude for the formation reaction $\bar{p} p \rightarrow \chi_{2}$ is given by:

$$
A\left(\bar{p} p \rightarrow \chi_{2}\right)=\left\langle 2, M|A| \theta, \phi ; \lambda_{1}, \lambda_{2}\right\rangle=\left(\frac{5}{4 \pi}\right)^{1 / 2} A_{\lambda_{1}, \lambda_{2}} \mathcal{D}_{M, \lambda}^{2}(\phi, \theta,-\phi)
$$

where $A_{\lambda_{1}, \lambda_{2}}=\left\langle 2, M|A| 2, M ; \lambda_{1}, \lambda_{2}\right\rangle$. Here, the definition of $D$ is useful to recall. It is

$$
\mathcal{D}_{m_{1}, m_{2}}^{J}(\alpha, \beta, \gamma)=e^{-i m_{1} \alpha} d_{m_{1}, m_{2}}^{J}(\beta) e^{-i m_{2} \gamma}
$$

We can choose our axes such that $\phi=0$, which allowing us to write Eq. C.4 in terms of the $d$ functions alone (which can be found in Ref. [2]):

$$
A\left(\bar{p} p \rightarrow \chi_{2}\right)=\left(\frac{5}{4 \pi}\right)^{1 / 2} A_{\lambda_{1}, \lambda_{2}} d_{M, \lambda}^{2}(\theta)
$$

$\underline{\chi_{2} \rightarrow \gamma \gamma}$

Following reasoning similar to that in the above section, we can write the amplitude for the decay process as (again, refer to Fig. C.1 for definitions):

$$
\begin{aligned}
A\left(\chi_{2} \rightarrow \gamma \gamma\right) & =\left\langle\theta^{*}, \phi^{*} ; \kappa_{1}, \kappa_{2}|B| 2, M\right\rangle \\
& =\left(\frac{5}{4 \pi}\right)^{1 / 2} B^{*}{ }_{\kappa_{1}, \kappa_{2}} \mathcal{D}^{* 2}{ }_{M, \kappa}\left(\phi^{*}, \theta^{*},-\phi^{*}\right)
\end{aligned}
$$

where $\kappa=\kappa_{1}-\kappa_{2}$ and the angles $\theta^{*}$ and $\phi^{*}$ are CM angles for one of the two photons.

The evaluation of this amplitude is simpler than the previous one, for the $\chi_{2}$ quantization axis can be chosen to lie along the line formed by the two photon momenta (thus $\theta^{*}=0$ ). Then, angular momentum conservation requires $\kappa=M$. 
and thus

$$
\left\langle\theta^{*}, \phi^{*} ; \kappa_{1}, \kappa_{2}|B| 2, M\right\rangle=\left(\frac{5}{4 \pi}\right)^{1 / 2} B_{\kappa_{1}, \kappa_{2}}^{*} \mathcal{D}_{M, M}^{* 2}\left(\phi^{*}, 0,-\phi^{*}\right)
$$

Again using Eq. C.5, we find that $\mathcal{D}_{M, \kappa}^{* 2}\left(\phi^{*}, 0,-\phi^{*}\right)=d_{M, M}^{2}(0)=\delta_{M, M}=1$. Thus

$$
\left\langle\theta^{*}, \phi^{*} ; \kappa_{1}, \kappa_{2}|B| 2, M\right\rangle=\left(\frac{5}{4 \pi}\right)^{1 / 2} B^{*}{ }_{\kappa_{1}, \kappa_{2}} .
$$

\section{$\underline{\bar{p} p \rightarrow \chi_{2} \rightarrow \gamma \gamma}$}

The amplitude for the complete reaction $\bar{p} p \rightarrow \chi_{2} \rightarrow \gamma \gamma$ is simply given by the product of the partial amplitudes, i.e.

$$
A\left(\bar{p} p \rightarrow \chi_{2} \rightarrow \gamma \gamma\right)=\frac{5}{4 \pi} B^{*}{ }_{\kappa_{1}, \kappa_{2}} A_{\lambda_{1}, \lambda_{2}} d_{M, \lambda}^{2}
$$

Our goal is the calculation of the angular distribution for this process. We can derive it from the differential cross section, which follows in a straightforward manner from the amplitude, averaging over initial spins and summing over the final spins:

$$
\frac{d \sigma}{d \Omega}\left(\bar{p} p \rightarrow \chi_{2} \rightarrow \gamma \gamma\right) \propto \frac{1}{4} \sum_{\kappa_{1}, \kappa_{2}} \sum_{\lambda_{1}, \lambda_{2}}\left|A\left(\bar{p} p \rightarrow \chi_{2} \rightarrow \gamma \gamma\right)\right|^{2}
$$

Evaluating the amplitudes

In order to determine $d \sigma / d \Omega$, we must evaluate the amplitudes $A_{\lambda_{1}, \lambda_{2}}$ and $B_{\kappa_{1}, \kappa_{2}}$. The number of amplitudes is small (8), and further, the strong and electromagnetic interactions which govern the $\bar{p} p \rightarrow \chi_{2} \rightarrow \gamma \gamma$ process respect both time reversal and parity invariance. These lead (see, e.g. Ref. [104]) to a reduction by a factor of two in the number of independent amplitudes:

$$
A_{\lambda_{1}, \lambda_{2}}=A_{-\lambda_{1},-\lambda_{2}} \text { and } B_{\kappa_{1}, \kappa_{2}}=B_{-\kappa_{1},-\kappa_{2}}
$$


As a result, we may specify the amplitudes by the value of the initial and final state helicities: the initial state helicity may be 0 or 1 (i.e. $\lambda_{1}$ and $\lambda_{2}$ may take values of $\pm 1 / 2$ ), and the final state helicity may be 0 or 2 (since real photons may have only helicities \pm 1 ). Thus, we use the following notation:

$$
\begin{array}{cc}
A_{0}=A_{ \pm 1 / 2, \mp 1 / 2} & A_{1}=A_{ \pm 1 / 2, \pm 1 / 2} \\
B_{0}=B_{ \pm 1, \mp 1} & B_{2}=B_{ \pm 1, \pm 1}
\end{array}
$$

Now, using the properties of the $d$ function, namely,

$$
d_{m, n}^{J}=(-1)^{n-m} d_{n, m}^{J}=d_{-n,-m}^{J}
$$

we obtain

$$
\begin{aligned}
\frac{d \sigma}{d \Omega} & \propto A_{0}^{2}\left[B_{0}^{2}\left(d_{0,0}^{2}(\theta)\right)^{2}+B_{2}^{2}\left(d_{2,0}^{2}(\theta)\right)^{2}\right] \\
& +A_{1}^{2}\left[B_{0}^{2}\left(d_{1,0}^{2}(\theta)\right)^{2}+B_{2}^{2}\left\{\frac{1}{2}\left(d_{2,1}^{2}(\theta)\right)^{2}+\frac{1}{2}\left(d_{2,-1}^{2}(\theta)\right)\right\}^{2}\right] .
\end{aligned}
$$

The relevant $d$ functions are: [2]

$$
\begin{aligned}
d_{0,0}^{2}(\theta) & =\frac{3}{2} \cos ^{2} \theta-\frac{1}{2} \\
d_{1,0}^{2}(\theta) & =-\sqrt{\frac{3}{2}} \sin \theta \cos \theta \\
d_{2,0}^{2}(\theta) & =\frac{\sqrt{6}}{4} \sin ^{2} \theta \\
d_{2, \pm 1}^{2}(\theta) & =-\left(\frac{1 \pm \cos \theta}{2}\right) \sin \theta
\end{aligned}
$$

Substituting these into Eq. C.17, we find:

$$
\begin{aligned}
\frac{d \sigma}{d \Omega} \propto & \left(18 A_{0}^{2} B_{0}^{2}+3 A_{0}^{2} B_{0}^{2}-12 A_{1}^{2} B_{0}^{2}-2 A_{1}^{2} B_{2}^{2}\right) \times \cos ^{4} \theta \\
& \left(12 A_{1}^{2} B_{0}^{2}-6 A_{0}^{2} B_{2}^{2}-6 A_{0}^{2} B_{0}^{2}\right) \times \cos ^{2} \theta \\
& \left(2 A_{0}^{2} B_{0}^{2}+3 A_{0}^{2} B_{2}^{2}+2 A_{1}^{2} B_{2}^{2}\right)
\end{aligned}
$$




\section{$\underline{\text { Helicity states contributing to the process } \bar{p} p \rightarrow \chi_{2} \rightarrow \gamma \gamma}$}

As noted earlier, there are two possible helicities for the initial state ( 0 or 1$)$ and two for the final state (0 or 2). It has been shown in both E760 and E835, using data for the $\bar{p} p \rightarrow \chi_{2} \rightarrow \gamma J / \psi$ reaction, that the initial state of $\bar{p}$ and $p$ predominantly is helicity 1. A $90 \%$ CL upper limit of $26 \%$ has been placed on the helicity 0 component. [52] The $\chi_{2} \rightarrow \gamma \gamma$ decay is predicted to be primarily helicity 2 , with at most a $5 \%$ contribution from helicity $0 .[53]$

If we assume purely helicity 1 formation and helicity 2 decay, we have $A_{0}^{2}=$ $B_{0}^{2}=0$, and the differential cross section becomes:

$$
\frac{d \sigma}{d \Omega}=A_{1}^{2} B_{2}^{2}\left(1-\cos ^{4} \theta\right)
$$

The angular distribution under this assumption, then, is

$$
W(\theta)=\mathcal{N}\left(1-\cos ^{4} \theta\right)
$$

The normalization factor $\mathcal{N}$ is obtained by setting the integral $\int_{0}^{1} W(\theta) d(\cos \theta)$ to 1. This leads to

$$
W(\theta)=\frac{5}{4}\left(1-\cos ^{4} \theta\right) .
$$

We assumed this angular distribution in calculating the efficiency of the cut in $\cos \theta^{*}$ for $\bar{p} p \rightarrow \chi_{2} \rightarrow \gamma \gamma$. 


\section{Appendix D}

\section{Statistical Considerations}

\section{Statistical Errors}

It is standard practice to calculate errors on counts according to Poisson statistics. The confidence limits at various levels: $68.27 \%, 90 \%, 95 \%, 99 \%$, etc., have been recently tabulated by Feldman and Cousins [108] for total number of events observed, $n_{\text {obs }}=0$ to 20 , with known mean background levels of 0 to 15 . Throughout this dissertation, we use these tables to obtain standard $68.27 \%$ statistical errors when the total number of counts in any measurement is less than 20 . When the total number of events is greater than 20, the Poisson distribution is well approximated by the Normal distribution, and the statistical error on $n_{o b s}$ is taken as $\sqrt{n_{o b s}}$. 


\section{Propagation of Errors}

Generally, statistical errors and systematic errors are propagated separately. For the propagation of statistical errors, the usual procedures are followed when the fractional errors are small : $\epsilon(a \pm b)=\sqrt{\epsilon^{2}(a)+\epsilon^{2}(b)}, f \epsilon(a / b, a b)=\sqrt{f \epsilon^{2}(a)+f \epsilon^{2}(b)}$, $f \epsilon\left(a^{n}\right)=n \times f \epsilon(a)$, where $\epsilon$ and $f \epsilon$ denote absolute and fractional errors, respectively.

When fractional errors are large, these simple expressions are not correct. The correct procedure often results in asymmetric errors on a function of $(a, b)$ even when the individual errors on $a$ and $b$ are each symmetric. As an example, if $a$ has symmetric errors of $\pm 30 \%, 1 / a$ acquires asymmetric errors of ${ }_{-23.1 \%}^{+42.8 \%}$.

The fact that this method is proper may be illustrated as follows. A Gaussian distribution for $x$ is shown in Fig. D.1. $68.3 \%$ of the area beneath the curve is found between the mean $\mu$ and $\mu \pm \sigma$. This function has a variance $\sigma$ which is $30 \%$ of the mean $\mu$. The distribution for $1 / x$, also shown in Fig. D.1, illustrates the problem. While the mean of the distribution is indeed $1 / \mu$, the shape of the distribution is highly asymmetric. Upon integrating this curve, we find that $68.3 \%$ of the area lies between $1 /(\mu+\sigma)$ and $1 /(\mu-\sigma)$. Thus we would properly quote the $68.3 \%$ confidence interval for $1 / x$ as $[1 /(\mu+\sigma), 1 /(\mu-\sigma)]$. As mentioned above, if $\sigma=0.3 \times \mu$, the errors on $1 / x$ are $+42.8 \%$ and $-23.1 \%$.

Therefore, when we calculate the branching ratio $B(c \bar{c} \rightarrow \gamma \gamma)$ from our determination of the branching ratio product $B(c \bar{c} \rightarrow p \bar{p}) \times B(c \bar{c} \rightarrow \gamma \gamma)$ by dividing by $B(c \bar{c} \rightarrow \bar{p} p)$ with large fractional error, we quote errors on $B(c \bar{c} \rightarrow \gamma \gamma)$ with these considerations in mind. 


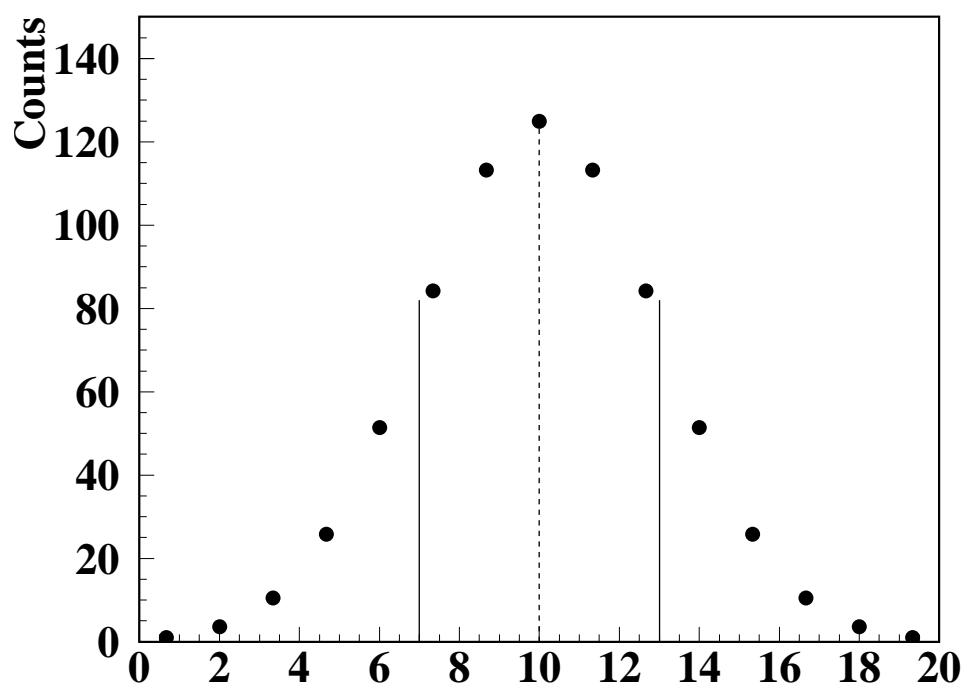

(x)

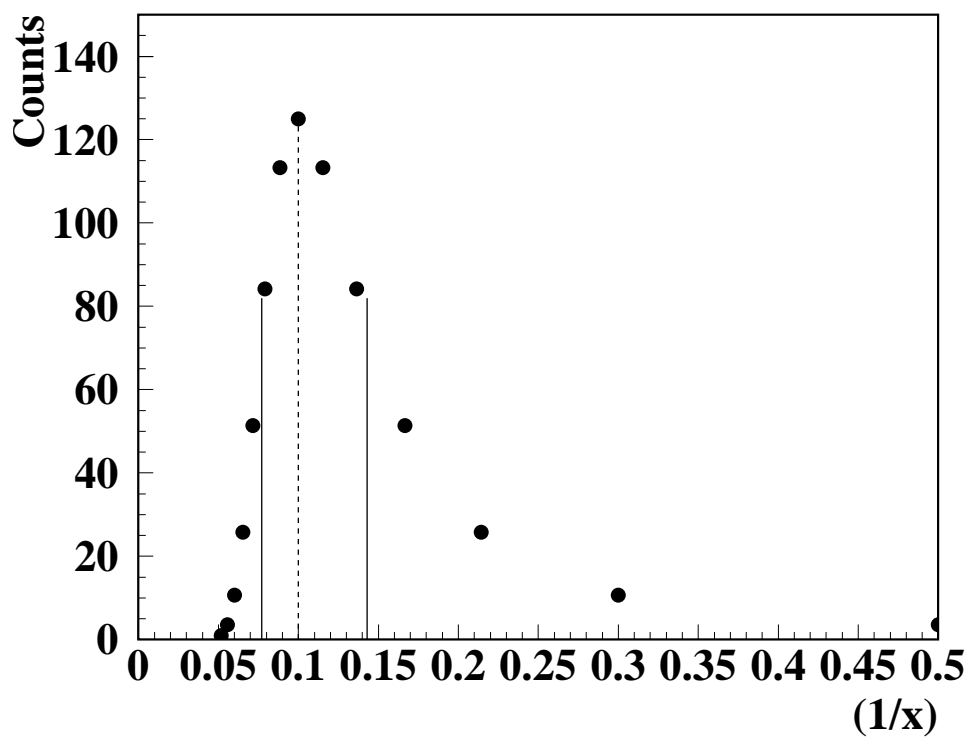

Figure D.1: (top) Gaussian distribution for $x$, with mean $\mu=10$ and variance $\sigma=3$. (bottom) Distribution of $1 / x$, for the same variable $x$. 
Almost by definition, one does not know how to combine different systematic errors. Sometimes these are combined linearly, but the general practice is to combine them in quadrature. We have followed this latter practice.

Sometimes when one is faced with assigning the total uncertainty in a result, one has the problem of combining statistical and systematic errors. Obviously, there can be no correct procedure for it. One can be very conservative and combine the two linearly or in quadrature. We have generally avoided the problem by specifying statistical and systematic errors separately. In rare cases, we have combined the two in quadrature.

\section{Estimation of Errors in Fits to Data with Small Statistics}

When the cross section data in the region of a resonance are fitted to a background plus a Breit-Wigner resonance, the MINUIT fit returns an estimate of the (generally) asymmetric errors. The question arises whether these errors represent the best estimate of the errors on the fit parameters. Most of the time one assumes that the Likelihood function is Gaussian. This may be a poor approximation in the case of small statistics. Thus the standard errors which are quoted by a fitting package such as MINUIT may not accurately reflect the true errors on the fit parameters. The problem becomes more acute when even the existence of the resonance is put in question. In such a case it is standard practice to quote an upper limit at a specific confidence level, for example, $90 \%$ CL. In this case, two procedures are possible.

Calculating Errors from the Likelihood Function

The first procedure is to use the results of the fit for a parameter, $\mu_{-\sigma_{2}}^{+\sigma_{1}}$, which 
imply a $68.27 \%$ confidence interval, $\left(\mu-\sigma_{2}, \mu+\sigma_{1}\right)$, and quote the $90 \%$ confidence upper limit as $\mu+1.28 \times \sigma_{1}$. For a single free parameter, the calculation of these errors $\sigma_{1}$ and $\sigma_{2}$ by may be done as follows. With just one free parameter $x$, the likelihood is a function only of $x$. Thus $L(x)$ may be calculated for a large range of $x$, and integrated to determine limits and/or errors for $x$.

For example, the $90 \%$ confidence upper limit on $x$ corresponds to the value $x_{90 \%}$ for which the following relation holds:

$$
\mathcal{A}_{90 \%}=\int_{-\infty}^{x_{90 \%}} L(x) d x=90 \% \times \int_{-\infty}^{+\infty} L(x) d x
$$

This procedure does not require $L(x)$ to be Gaussian, and thus the correct upper limit $x_{90 \%}$ is obtained.

The only question about this method is whether one should integrate the Likelihood function over all values of $x$, even if the Likelihood function has finite value in regions of $x$ which are not physically allowed. This question is somewhat unsettled, as discussed in Ref. [108]. In both the $\chi_{0}$ and $\eta_{c}^{\prime}$ analyses, in which we follow the above procedure for calculating $90 \%$ upper limits, we have tabulated both the mean value $\mu$ and the $90 \%$ upper limit so that the reader can calculate upper limits himself using a different procedure. (See Table D.1)

$\underline{\text { Estimating Errors by Monte Carlo Techniques }}$

The other procedure is to recognize that it is incorrect to assume that the actual small statistics experiment can tell us the correct errors at all, and that one should 'repeat' the small statistics experiment a large number of times to determine the variability of the results, and assign errors and upper limits accordingly. Obviously, the large number of "repeat" experiments can be done only in Monte Carlo 
simulations. An example will illustrate the method.

Using the central values of best fit resonance and background parameters obtained in our analysis of the $\eta_{c}$ data,

$$
\begin{array}{cc}
M\left(\eta_{c}\right)=2982.4_{-2.4}^{+2.2} \mathrm{MeV} & \Gamma\left(\eta_{c}\right)=26.9_{-8.5}^{+12.1} \mathrm{MeV} \\
B\left(\eta_{c} \rightarrow \bar{p} p\right) \times \Gamma\left(\eta_{c} \rightarrow \gamma \gamma\right)=6.8_{-2.4}^{+4.0} \mathrm{eV} \\
A_{b k g}=12.5_{-7.3}^{+11.41} \mathrm{pb} & B_{b k g}=59.0_{-22.5}^{+39.2} \\
C_{b k g}=7.67_{-0.41}^{+0.43} \mathrm{pb} & D_{b k g}=8.80_{-1.58}^{+1.27}
\end{array}
$$

and our overall efficiency, acceptance and luminosity, we first calculated the expected number of events at each of the 146 energy points from 2911 to $3829 \mathrm{MeV}$.

We then run 10,000 Monte Carlo "experiments". In each "experiment" we obtain a number of observed events at each E835 energy point, according to the appropriate Poisson distribution for the expected mean number of events calculated above. Each MC "experimental" spectrum is then fit for the resonance and background parameters, exactly as the experimental data was.

The distribution of the 10,000 results for the parameters $M\left(\eta_{c}\right), \Gamma\left(\eta_{c}\right)$ and $B\left(\eta_{c} \rightarrow \bar{p} p\right) \times \Gamma\left(\eta_{c} \rightarrow \gamma \gamma\right)$ reflect the correct distribution functions for these parameters, from which true errors may be obtained. As an example, in Fig. D.2 we show the result of the fits for $\Gamma\left(\eta_{c}\right)$. From this obviously asymmetric distribution, we can obtain the positive error on $\Gamma\left(\eta_{c}\right)$ by integrating the distribution up from the mean value $\Gamma_{0}\left(\eta_{c}\right)$ until $68.3 \%$ of the area above the mean is covered. We obtain the negative error similarly. This procedure obtains the $\pm 68.3 \%$ confidence interval. In the case of $\Gamma\left(\eta_{c}\right)$, we find that the result for the total width $\Gamma\left(\eta_{c}\right)$ is $26.9_{-9.5}^{+10.8}$ MeV. Note that this is different, but not substantially so, than the errors in Eq. D.2 


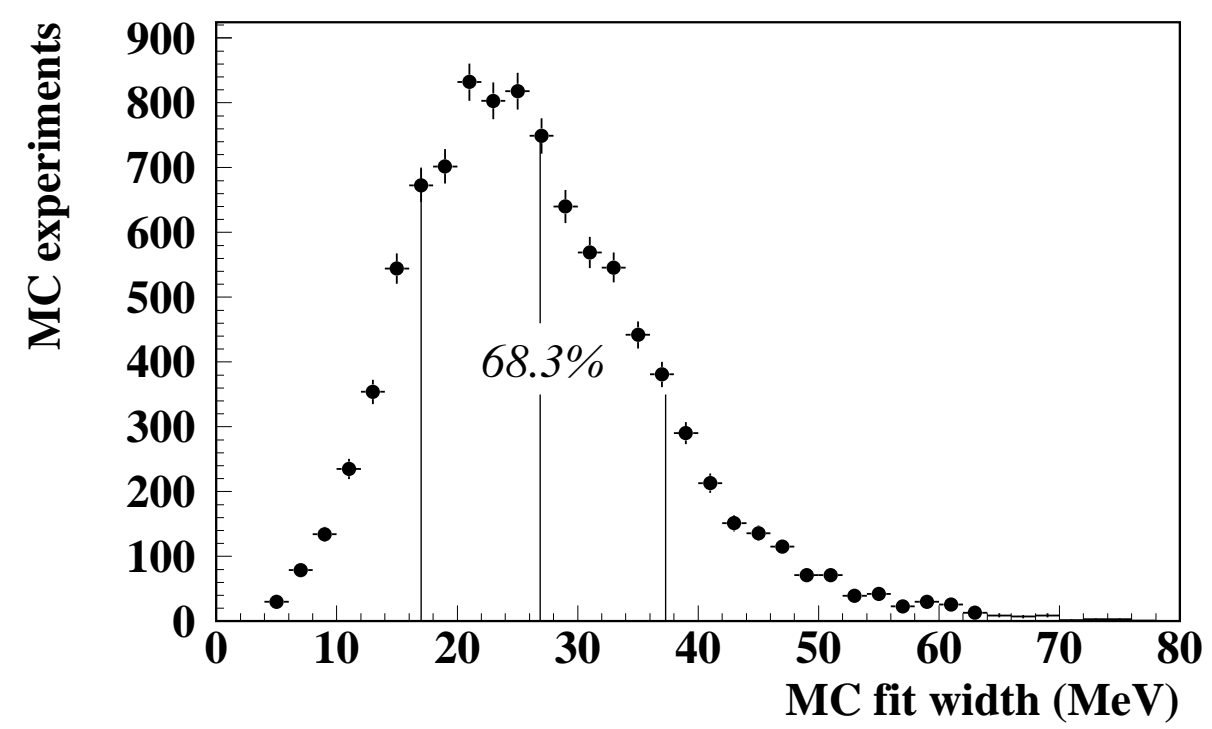

Figure D.2: Result of fits to $10,000 \mathrm{MC}$ experiments for $\Gamma\left(\eta_{c}\right)$. The lines indicate the limits of the standard $(68.3 \%)$ confidence interval. From this distribution we obtain errors on the total width of $(+10.8)$ and $(-9.5) \mathrm{MeV}$.

determined by the fit. The errors for the other resonance parameters for $\eta_{c}$ have been obtained in a similar manner. 
Table D.1: Results of fits to $\eta_{c}^{\prime}$ data. The Mass column indicates the assumed mass for the fit. The three pairs of columns indicate the mean value of $B\left(\eta_{c}^{\prime} \rightarrow \bar{p} p\right) \times$ $B\left(\eta_{c}^{\prime} \rightarrow \gamma \gamma\right)$ and the $90 \%$ confidence upper limit, derived from the likelihood function.

\begin{tabular}{|l|ll|ll|ll|}
\hline & \multicolumn{2}{|c|}{$\Gamma\left(\eta_{c}^{\prime}\right)=10 \mathrm{MeV}$} & \multicolumn{2}{|c|}{$\Gamma\left(\eta_{c}^{\prime}\right)=15 \mathrm{MeV}$} & \multicolumn{2}{|c|}{$\Gamma\left(\eta_{c}^{\prime}\right)=20 \mathrm{MeV}$} \\
\cline { 2 - 7 }$(\mathrm{MeV})$ & Mean & $90 \% \mathrm{CL}$ & Mean & $90 \% \mathrm{CL}$ & Mean & $90 \% \mathrm{CL}$ \\
& & upper limit & & upper limit & & upper limit \\
\hline 3575 & -1.2498 & 1.1121 & -0.8510 & 1.2167 & -0.6101 & 1.2283 \\
3576 & -1.0518 & 1.1530 & -0.7324 & 1.2233 & -0.5395 & 1.2188 \\
3577 & -0.8443 & 1.3071 & -0.6192 & 1.2613 & -0.4726 & 1.2220 \\
3579 & -0.6038 & 1.5370 & -0.5129 & 1.3171 & -0.4118 & 1.2342 \\
3580 & -0.3721 & 1.7579 & -0.4218 & 1.3742 & -0.3580 & 1.2509 \\
3581 & -0.2214 & 1.8976 & -0.3550 & 1.4186 & -0.3125 & 1.2682 \\
3582 & -0.2582 & 1.8791 & -0.3039 & 1.4455 & -0.2451 & 1.2977 \\
3583 & -0.3893 & 1.7449 & -0.3061 & 1.4351 & -0.2190 & 1.3107 \\
3584 & -0.5096 & 1.5957 & -0.3098 & 1.4234 & -0.1934 & 1.3254 \\
3585 & -0.5706 & 1.5088 & -0.3027 & 1.4233 & -0.1651 & 1.3444 \\
3586 & -0.5626 & 1.5232 & -0.2777 & 1.4425 & -0.1319 & 1.3694 \\
3587 & -0.4828 & 1.6228 & -0.2337 & 1.4810 & -0.0928 & 1.4011 \\
3588 & -0.3491 & 1.7500 & -0.1759 & 1.5327 & -0.0483 & 1.4388 \\
3589 & -0.2125 & 1.8526 & -0.1119 & 1.5906 & 0.0005 & 1.4815 \\
3590 & -0.1140 & 1.9304 & -0.0488 & 1.6507 & 0.0524 & 1.5278 \\
3591 & -0.0594 & 2.0074 & 0.0111 & 1.7115 & 0.1069 & 1.5770 \\
3592 & -0.0374 & 2.0809 & 0.0695 & 1.7723 & 0.1637 & 1.6282 \\
3593 & -0.0294 & 2.1220 & 0.1303 & 1.8329 & 0.2233 & 1.6810 \\
3594 & -0.0079 & 2.1289 & 0.1986 & 1.8948 & 0.2860 & 1.7350 \\
3595 & 0.0558 & 2.1547 & 0.2785 & 1.9625 & 0.3517 & 1.7899 \\
3596 & 0.1826 & 2.2591 & 0.3719 & 2.0389 & 0.4192 & 1.8449 \\
3597 & 0.3770 & 2.4453 & 0.4750 & 2.1216 & 0.4873 & 1.8987 \\
3598 & 0.6039 & 2.6465 & 0.5798 & 2.2025 & 0.5530 & 1.9497 \\
\hline & & & & & $C o n t i n u e d$ & on 2 next page \\
\hline
\end{tabular}




\begin{tabular}{|c|c|c|c|c|c|c|}
\hline \multicolumn{7}{|c|}{ Results for $B\left(\eta_{c}^{\prime} \rightarrow \bar{p} p\right) \times B\left(\eta_{c}^{\prime} \rightarrow \gamma \gamma\right)\left(\times 10^{8}\right)$. Continued from previous page } \\
\hline \multirow[b]{2}{*}{$\begin{array}{l}\text { Mass } \\
(\mathrm{MeV})\end{array}$} & \multicolumn{2}{|c|}{$\Gamma\left(\eta_{c}^{\prime}\right)=10 \mathrm{MeV}$} & \multicolumn{2}{|c|}{$\Gamma\left(\eta_{c}^{\prime}\right)=15 \mathrm{MeV}$} & \multicolumn{2}{|c|}{$\Gamma\left(\eta_{c}^{\prime}\right)=20 \mathrm{MeV}$} \\
\hline & Mean & $\begin{array}{l}90 \% \text { CL } \\
\text { upper limit }\end{array}$ & Mean & $\begin{array}{l}90 \% \text { CL } \\
\text { upper limit }\end{array}$ & Mean & $\begin{array}{l}90 \% \text { CL } \\
\text { upper limit }\end{array}$ \\
\hline 3599 & 0.8038 & 2.7906 & 0.6755 & 2.2732 & 0.6141 & 1.9963 \\
\hline 3600 & 0.9517 & 2.8751 & 0.7570 & 2.3286 & 0.6679 & 2.0379 \\
\hline 3601 & 1.0578 & 2.9357 & 0.8182 & 2.3692 & 0.7153 & 2.0739 \\
\hline 3602 & 1.1257 & 2.9761 & 0.8586 & 2.3960 & 0.7545 & 2.1057 \\
\hline 3603 & 1.1361 & 2.9722 & 0.8780 & 2.4088 & 0.7871 & 2.1350 \\
\hline 3604 & 1.0788 & 2.9137 & 0.8789 & 2.4113 & 0.8154 & 2.1642 \\
\hline 3605 & 0.9556 & 2.8037 & 0.8665 & 2.4086 & 0.8430 & 2.1970 \\
\hline 3606 & 0.7734 & 2.6473 & 0.8519 & 2.4109 & 0.8747 & 2.2376 \\
\hline 3607 & 0.5699 & 2.4788 & 0.8509 & 2.4337 & 0.9170 & 2.2901 \\
\hline 3608 & 0.4125 & 2.3747 & 0.8845 & 2.4957 & 0.9737 & 2.3590 \\
\hline 3609 & 0.3874 & 2.4269 & 0.9734 & 2.6137 & 1.0475 & 2.4460 \\
\hline 3610 & 0.6007 & 2.7228 & 1.1267 & 2.7937 & 1.1396 & 2.5489 \\
\hline 3611 & 1.1072 & 3.2852 & 1.3370 & 3.0241 & 1.2434 & 2.6626 \\
\hline 3612 & 1.7901 & 3.9860 & 1.5761 & 3.2779 & 1.3523 & 2.7781 \\
\hline 3613 & 2.4355 & 4.6297 & 1.8113 & 3.5218 & 1.4537 & 2.8856 \\
\hline 3614 & 2.9284 & 5.1260 & 2.0105 & 3.7298 & 1.5400 & 2.9745 \\
\hline 3615 & 3.2730 & 5.5033 & 2.1538 & 3.8833 & 1.6006 & 3.0367 \\
\hline 3616 & 3.4827 & 5.7740 & 2.2296 & 3.9674 & 1.6300 & 3.0656 \\
\hline 3617 & 3.5024 & 5.8505 & 2.2275 & 3.9704 & 1.6251 & 3.0574 \\
\hline 3618 & 3.2679 & 5.6294 & 2.1476 & 3.8878 & 1.5858 & 3.0116 \\
\hline 3619 & 2.8327 & 5.1517 & 2.0034 & 3.7301 & 1.5159 & 2.9311 \\
\hline 3620 & 2.3625 & 4.6050 & 1.8171 & 3.5208 & 1.4215 & 2.8217 \\
\hline 3621 & 1.9673 & 4.1431 & 1.6100 & 3.2855 & 1.3096 & 2.6911 \\
\hline 3622 & 1.6428 & 3.7689 & 1.4011 & 3.0404 & 1.1881 & 2.5468 \\
\hline 3623 & 1.3356 & 3.4059 & 1.1944 & 2.7949 & 1.0622 & 2.3960 \\
\hline 3624 & 1.0292 & 3.0268 & 0.9968 & 2.5545 & 0.9391 & 2.2441 \\
\hline 3625 & 0.7532 & 2.6725 & 0.8134 & 2.3252 & 0.8223 & 2.0968 \\
\hline 3626 & 0.5168 & 2.3698 & 0.6486 & 2.1121 & 0.7160 & 1.9586 \\
\hline 3627 & 0.3039 & 2.0938 & 0.5063 & 1.9183 & 0.6234 & 1.8335 \\
\hline 3628 & 0.1145 & 1.8218 & 0.3890 & 1.7476 & 0.5473 & 1.7251 \\
\hline 3629 & -0.0282 & 1.5752 & 0.3010 & 1.6056 & 0.4900 & 1.6369 \\
\hline 3630 & -0.1110 & 1.3854 & 0.2452 & 1.4987 & 0.4522 & 1.5718 \\
\hline & & & & & ntinued & on next page \\
\hline
\end{tabular}




\begin{tabular}{|c|c|c|c|c|c|c|}
\hline \multicolumn{7}{|c|}{ Results for $B\left(\eta_{c}^{\prime} \rightarrow \bar{p} p\right) \times B\left(\eta_{c}^{\prime} \rightarrow \gamma \gamma\right)\left(\times 10^{8}\right)$. Continued from previous page } \\
\hline \multirow[b]{2}{*}{$\begin{array}{l}\text { Mass } \\
(\mathrm{MeV})\end{array}$} & \multicolumn{2}{|c|}{$\Gamma\left(\eta_{c}^{\prime}\right)=10 \mathrm{MeV}$} & \multicolumn{2}{|c|}{$\Gamma\left(\eta_{c}^{\prime}\right)=15 \mathrm{MeV}$} & \multicolumn{2}{|c|}{$\Gamma\left(\eta_{c}^{\prime}\right)=20 \mathrm{MeV}$} \\
\hline & Mean & $\begin{array}{l}90 \% \text { CL } \\
\text { upper limit }\end{array}$ & Mean & $\begin{array}{l}90 \% \text { CL } \\
\text { upper limit }\end{array}$ & Mean & $\begin{array}{l}90 \% \text { CL } \\
\text { upper limit }\end{array}$ \\
\hline 3631 & -0.1389 & 1.2605 & 0.2226 & 1.4324 & 0.4357 & 1.5323 \\
\hline 3632 & -0.1198 & 1.2005 & 0.2335 & 1.4115 & 0.4399 & 1.5201 \\
\hline 3633 & -0.0546 & 1.2206 & 0.2784 & 1.4406 & 0.4650 & 1.5358 \\
\hline 3634 & 0.0679 & 1.3491 & 0.3593 & 1.5236 & 0.5101 & 1.5791 \\
\hline 3635 & 0.2706 & 1.6144 & 0.4775 & 1.6612 & 0.5727 & 1.6474 \\
\hline 3636 & 0.5762 & 2.0284 & 0.6311 & 1.8471 & 0.6495 & 1.7360 \\
\hline 3637 & 0.9792 & 2.5549 & 0.8099 & 2.0642 & 0.7355 & 1.8373 \\
\hline 3638 & 1.4099 & 3.0850 & 0.9946 & 2.2861 & 0.8231 & 1.9424 \\
\hline 3639 & 1.7664 & 3.4962 & 1.1620 & 2.4847 & 0.9038 & 2.0424 \\
\hline 3640 & 1.9960 & 3.7423 & 1.2926 & 2.6403 & 0.9725 & 2.1289 \\
\hline 3641 & 2.1023 & 3.8384 & 1.3796 & 2.7448 & 1.0219 & 2.1976 \\
\hline 3642 & 2.1013 & 3.8170 & 1.4203 & 2.8034 & 1.0496 & 2.2461 \\
\hline 3643 & 2.0334 & 3.7376 & 1.4210 & 2.8263 & 1.0544 & 2.2741 \\
\hline 3644 & 1.9528 & 3.6811 & 1.3863 & 2.8237 & 1.0354 & 2.2816 \\
\hline 3645 & 1.8759 & 3.6890 & 1.3141 & 2.7974 & 0.9907 & 2.2676 \\
\hline 3646 & 1.7544 & 3.7117 & 1.1955 & 2.7353 & 0.9176 & 2.2300 \\
\hline 3647 & 1.4725 & 3.6051 & 1.0113 & 2.6154 & 0.8161 & 2.1650 \\
\hline 3648 & 0.9064 & 3.1914 & 0.7494 & 2.4160 & 0.6842 & 2.0716 \\
\hline 3649 & 0.0645 & 2.4148 & 0.4171 & 2.1341 & 0.5289 & 1.9517 \\
\hline 3650 & -0.8109 & 1.4823 & 0.0493 & 1.7975 & 0.3601 & 1.8139 \\
\hline 3651 & -1.4512 & 0.7077 & -0.3000 & 1.4588 & 0.1929 & 1.6726 \\
\hline 3652 & -1.8522 & 0.2015 & -0.5824 & 1.1724 & 0.0447 & 1.5446 \\
\hline 3653 & -2.0950 & -0.0874 & -0.7688 & 0.9751 & -0.0691 & 1.4463 \\
\hline 3654 & -2.1601 & -0.1795 & -0.8505 & 0.8843 & -0.1385 & 1.3898 \\
\hline 3655 & -2.0136 & -0.0640 & -0.8256 & 0.9017 & -0.1595 & 1.3815 \\
\hline 3656 & -1.6953 & 0.2505 & -0.7074 & 1.0198 & -0.1341 & 1.4226 \\
\hline 3657 & -1.2225 & 0.7582 & -0.5178 & 1.2222 & -0.0686 & 1.5097 \\
\hline 3658 & -0.6233 & 1.4044 & -0.2828 & 1.4852 & 0.0280 & 1.6370 \\
\hline 3659 & -0.0218 & 2.0580 & -0.0303 & 1.7860 & 0.1467 & 1.7985 \\
\hline 3660 & 0.4738 & 2.6421 & 0.2187 & 2.1113 & 0.2802 & 1.9897 \\
\hline
\end{tabular}




\section{Appendix E}

\section{Summary of All $\gamma \gamma$ Data}

Table E.1: $\gamma \gamma$ candidate events, for $\left|\cos \theta^{*}\right| \leq 0.20$.

\begin{tabular}{|c|c|c|c|c|c|c|}
\hline $\begin{array}{c}\text { CM energy } \\
\sqrt{s} \\
(\mathrm{MeV})\end{array}$ & $\begin{array}{c}\text { Energy spread } \\
\sigma_{E} \\
(\mathrm{MeV})\end{array}$ & $\begin{array}{l}\text { 1st run } \\
\text { number }\end{array}$ & $N_{e v t}$ & $\begin{array}{l}\text { Luminosit } \\
\qquad \int \mathcal{L} \\
\mathrm{cm}^{-2} \mathrm{sec}^{-}\end{array}$ & $\begin{array}{c}\text { Efficiency } \\
\epsilon\end{array}$ & $\begin{array}{l}\sigma^{0.20} \\
(\mathrm{pb})\end{array}$ \\
\hline 2911.365 & 0.28610 & 2324 & 35 & 514.68 & 0.70984 & 95.801 \\
\hline 2912.259 & 0.24760 & 2295 & 15 & 190.21 & 0.74487 & 105.872 \\
\hline 2930.109 & 0.22000 & 2102 & 14 & 317.63 & 0.71778 & 61.407 \\
\hline 2930.482 & 0.17010 & 2042 & 20 & 372.28 & 0.71031 & 75.633 \\
\hline 2930.625 & 0.18170 & 2107 & 8 & 224.41 & 0.71218 & 50.057 \\
\hline 2930.753 & 0.25560 & 2330 & 49 & 855.31 & 0.67995 & 84.255 \\
\hline 2950.177 & 0.29460 & 1136 & 11 & 261.60 & 0.70190 & 59.907 \\
\hline 2950.710 & 0.18510 & 2098 & 23 & 406.10 & 0.69863 & 81.067 \\
\hline 2950.774 & 0.26960 & 2225 & 35 & 604.88 & 0.66454 & 87.072 \\
\hline 2951.131 & 0.27060 & 2175 & 20 & 373.33 & 0.73226 & 73.160 \\
\hline 2956.259 & 0.18000 & 2309 & 18 & 397.91 & 0.72572 & 62.333 \\
\hline 2956.290 & 0.24020 & 2314 & 54 & 979.22 & 0.67902 & 81.214 \\
\hline \multicolumn{7}{|c|}{ Continued on next page } \\
\hline
\end{tabular}




\begin{tabular}{|c|c|c|c|c|c|c|}
\hline \multicolumn{2}{|c|}{$\gamma \gamma$ candidate events, for } & \multicolumn{2}{|c|}{$\cos \theta^{*} \mid \leq 0.20$} & \multicolumn{3}{|c|}{ Continued from previous page } \\
\hline $\begin{array}{c}\text { CM energy } \\
\sqrt{s} \\
(\mathrm{MeV})\end{array}$ & $\begin{array}{c}\text { Energy spread } \\
\sigma_{E} \\
(\mathrm{MeV})\end{array}$ & $\begin{array}{l}\text { 1st run } \\
\text { number }\end{array}$ & $N_{e v t}$ & $\begin{array}{l}\text { Luminosity } \\
\qquad \mathrm{cm}^{-2} \mathrm{sec}^{-1}\end{array}$ & $\begin{array}{c}\text { Efficiency } \\
\epsilon\end{array}$ & $\begin{array}{l}\sigma^{0.20} \\
(\mathrm{pb})\end{array}$ \\
\hline 2966.866 & 0.23780 & 2259 & 30 & 587.86 & 0.68089 & 74.950 \\
\hline 2966.937 & 0.34740 & 1255 & 15 & 285.76 & 0.70891 & 74.046 \\
\hline 2970.145 & 0.17930 & 2086 & 22 & 401.54 & 0.72245 & 75.838 \\
\hline 2973.136 & 0.30220 & 2273 & 45 & 788.67 & 0.68416 & 83.399 \\
\hline 2973.970 & 0.21510 & 2052 & 41 & 619.80 & 0.68089 & 97.153 \\
\hline 2975.388 & 0.22740 & 2147 & 14 & 344.59 & 0.68089 & 59.669 \\
\hline 2980.475 & 0.17450 & 1299 & 30 & 440.73 & 0.72105 & 94.403 \\
\hline 2980.513 & 0.27870 & 1291 & 13 & 249.94 & 0.70937 & 73.322 \\
\hline 2983.052 & 0.28540 & 2359 & 66 & 1004.18 & 0.70003 & 93.889 \\
\hline 2985.989 & 0.18930 & 2139 & 33 & 467.67 & 0.71031 & 99.341 \\
\hline 2986.086 & 0.30270 & 2344 & 95 & 1410.42 & 0.67248 & 100.160 \\
\hline 2987.575 & 0.27530 & 1271 & 17 & 341.00 & 0.71124 & 70.093 \\
\hline 2987.749 & 0.19320 & 1093 & 11 & 249.58 & 0.71684 & 61.483 \\
\hline 2987.998 & 0.22880 & 2188 & 22 & 370.71 & 0.71778 & 82.679 \\
\hline 2990.660 & 0.24090 & 2162 & 32 & 499.08 & 0.71544 & 89.620 \\
\hline 2996.140 & 0.20240 & 2065 & 32 & 742.67 & 0.66594 & 64.702 \\
\hline 3000.857 & 0.25420 & 2155 & 19 & 347.31 & 0.70564 & 77.527 \\
\hline 3001.308 & 0.21170 & 2184 & 5 & 209.76 & 0.71311 & 33.427 \\
\hline 3004.917 & 0.31750 & 2298 & 40 & 1169.54 & 0.68649 & 49.821 \\
\hline 3009.094 & 0.25010 & 2120 & 30 & 929.27 & 0.69863 & 46.209 \\
\hline 3025.170 & 0.18670 & 1193 & 2 & 104.22 & 0.70704 & 27.142 \\
\hline 3026.103 & 0.24110 & 1209 & 13 & 489.33 & 0.70050 & 37.926 \\
\hline 3096.682 & 0.11360 & 3078 & 3 & 151.85 & 0.75841 & 26.050 \\
\hline 3097.068 & 0.16110 & 824 & 10 & 274.52 & 0.72759 & 50.066 \\
\hline 3097.137 & 0.14970 & 908 & 2 & 285.22 & 0.75000 & 9.349 \\
\hline 3097.255 & 0.25810 & 1331 & 7 & 491.92 & 0.73086 & 19.470 \\
\hline 3100.605 & 0.20100 & 3284 & 4 & 143.16 & 0.75280 & 37.116 \\
\hline 3215.712 & 0.36110 & 3206 & 7 & 419.93 & 0.68696 & 24.266 \\
\hline 3269.433 & 0.28740 & 3346 & 5 & 411.76 & 0.74673 & 16.261 \\
\hline & & & & & zued or & $x t$ page \\
\hline
\end{tabular}




\begin{tabular}{|c|c|c|c|c|c|c|}
\hline \multicolumn{4}{|c|}{$\gamma \gamma$ candidate events, for $\left|\cos \theta^{*}\right| \leq 0.20$} & \multicolumn{3}{|c|}{ Continued from previous page } \\
\hline $\begin{array}{c}\text { CM energy } \\
\sqrt{s} \\
(\mathrm{MeV})\end{array}$ & $\begin{array}{c}\text { Energy spread } \\
\sigma_{E} \\
(\mathrm{MeV})\end{array}$ & $\begin{array}{l}\text { 1st run } \\
\text { number }\end{array}$ & $N_{e v t}$ & $\begin{array}{l}\text { Luminosity } \\
\qquad \mathrm{cm}^{-2} \mathrm{sec}^{-1}\end{array}$ & $\begin{array}{c}\text { Efficiency } \\
\epsilon\end{array}$ & $\begin{array}{l}\sigma^{0.20} \\
(\mathrm{pb})\end{array}$ \\
\hline 3318.819 & 0.71580 & 3301 & 6 & 951.38 & 0.68556 & 9.199 \\
\hline 3405.813 & 0.47820 & 3278 & 2 & 80.67 & 0.66594 & 37.229 \\
\hline 3406.760 & 0.35630 & 3278 & 13 & 925.95 & 0.66594 & 21.082 \\
\hline 3414.783 & 0.35110 & 3270 & 7 & 584.95 & 0.62578 & 19.123 \\
\hline 3414.819 & 0.19710 & 3265 & 3 & 353.18 & 0.71871 & 11.819 \\
\hline 3418.135 & 0.90030 & 3076 & 2 & 146.25 & 0.73833 & 18.522 \\
\hline 3418.456 & 0.27800 & 3245 & 2 & 692.03 & 0.69723 & 4.145 \\
\hline 3429.533 & 0.58750 & 3316 & 3 & 348.75 & 0.74300 & 11.578 \\
\hline 3429.867 & 0.37780 & 3318 & 2 & 390.24 & 0.76635 & 6.688 \\
\hline 3494.432 & 0.33350 & 3341 & 5 & 502.79 & 0.67108 & 14.819 \\
\hline 3508.590 & 0.23210 & 3201 & 3 & 376.23 & 0.64353 & 12.391 \\
\hline 3509.217 & 0.18080 & 1431 & 0 & 303.97 & 0.71311 & 0.000 \\
\hline 3509.798 & 0.20450 & 1396 & 0 & 235.09 & 0.71404 & 0.000 \\
\hline 3509.932 & 0.25200 & 3198 & 0 & 317.08 & 0.65100 & 0.000 \\
\hline 3510.362 & 0.27850 & 3194 & 2 & 314.95 & 0.63745 & 9.962 \\
\hline 3510.405 & 0.27650 & 1318 & 4 & 1125.03 & 0.67155 & 5.294 \\
\hline 3510.539 & 0.24610 & 1427 & 2 & 314.50 & 0.68836 & 9.238 \\
\hline 3510.748 & 0.28990 & 3191 & 2 & 318.76 & 0.62531 & 10.034 \\
\hline 3510.779 & 0.25750 & 3286 & 3 & 780.02 & 0.68042 & 5.652 \\
\hline 3510.831 & 0.29490 & 3295 & 7 & 1147.06 & 0.62204 & 9.810 \\
\hline 3511.053 & 0.30790 & 3188 & 3 & 319.43 & 0.62765 & 14.963 \\
\hline 3511.172 & 0.21370 & 1394 & 1 & 218.01 & 0.72852 & 6.296 \\
\hline 3511.445 & 0.31510 & 3185 & 3 & 315.47 & 0.62998 & 15.095 \\
\hline 3511.752 & 0.20990 & 1424 & 2 & 319.68 & 0.67715 & 9.239 \\
\hline 3513.002 & 0.30390 & 3181 & 1 & 301.33 & 0.63932 & 5.191 \\
\hline 3524.642 & 0.30440 & 3081 & 24 & 3716.90 & 0.63092 & 10.234 \\
\hline 3525.161 & 0.28020 & 3037 & 18 & 2902.98 & 0.64213 & 9.656 \\
\hline 3525.505 & 0.28230 & 3103 & 16 & 3531.91 & 0.62158 & 7.288 \\
\hline 3525.637 & 0.34600 & 1414 & 7 & 1800.85 & 0.63979 & 6.076 \\
\hline
\end{tabular}




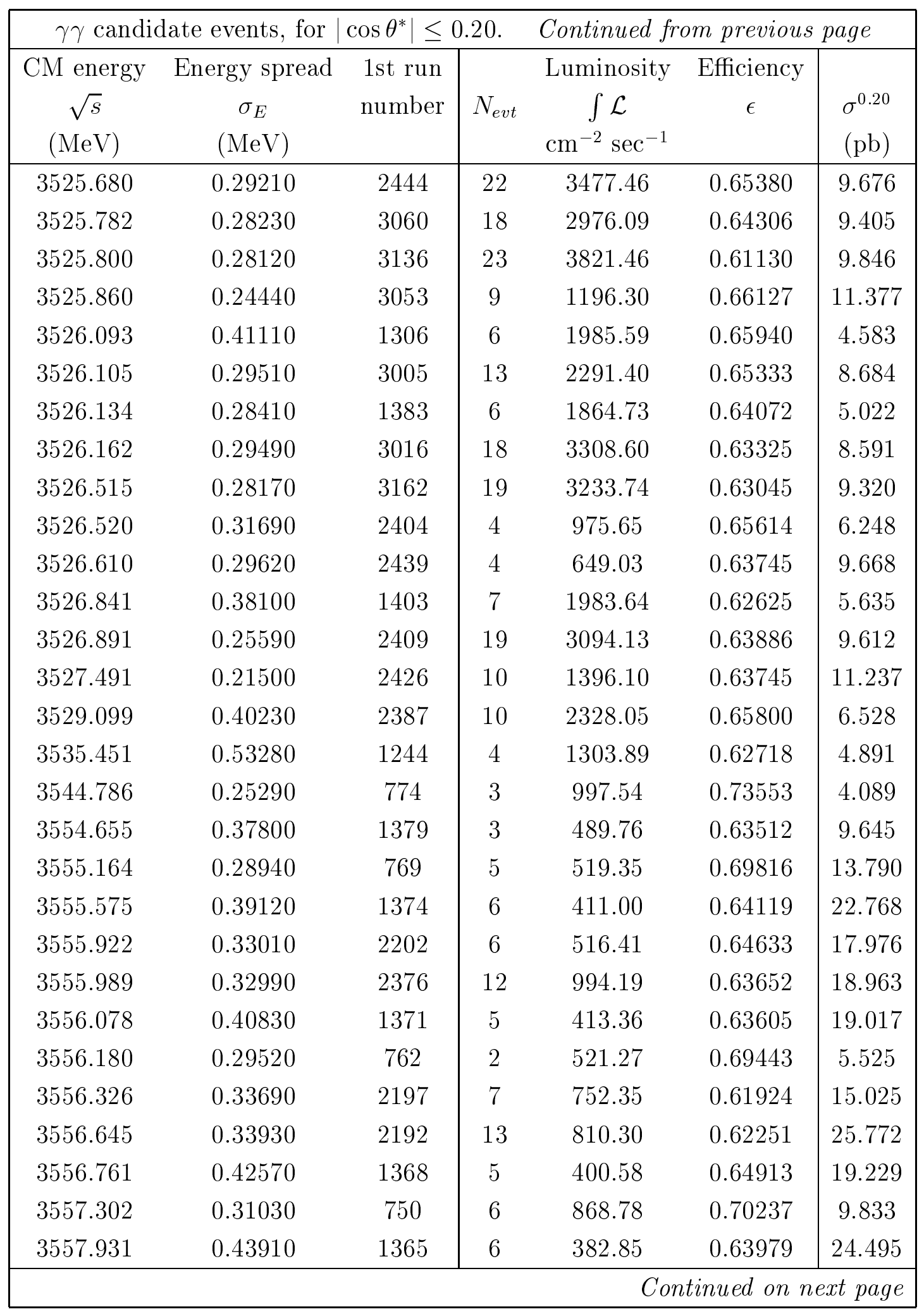




\begin{tabular}{|c|c|c|c|c|c|c|}
\hline \multicolumn{4}{|c|}{$\gamma \gamma$ candidate events, for $\left|\cos \theta^{*}\right| \leq 0.20$} & \multicolumn{3}{|c|}{ Continued from previous page } \\
\hline $\begin{array}{c}\text { CM energy } \\
\sqrt{s} \\
(\mathrm{MeV})\end{array}$ & $\begin{array}{c}\text { Energy spread } \\
\sigma_{E} \\
(\mathrm{MeV})\end{array}$ & $\begin{array}{l}\text { 1st run } \\
\text { number }\end{array}$ & $N_{e v t}$ & $\begin{array}{l}\text { Luminosity } \\
\qquad \mathrm{cm}^{-2} \mathrm{sec}^{-1}\end{array}$ & $\begin{array}{c}\text { Efficiency } \\
\epsilon\end{array}$ & $\begin{array}{l}\sigma^{0.20} \\
(\mathrm{pb})\end{array}$ \\
\hline 3555.259 & 0.32530 & 3339 & 4 & 501.02 & 0.66454 & 12.014 \\
\hline 3556.060 & 0.31550 & 1086 & 7 & 778.22 & 0.74113 & 12.137 \\
\hline 3576.054 & 0.25090 & 1283 & 8 & 1605.78 & 0.62531 & 7.967 \\
\hline 3580.087 & 0.36050 & 1132 & 6 & 582.80 & 0.66174 & 15.558 \\
\hline 3580.869 & 0.31880 & 1122 & 3 & 626.96 & 0.61457 & 7.786 \\
\hline 3585.185 & 0.35770 & 1186 & 4 & 1505.67 & 0.63839 & 4.161 \\
\hline 3590.246 & 0.35550 & 1112 & 7 & 1483.73 & 0.70424 & 6.699 \\
\hline 3595.616 & 0.33580 & 1126 & 7 & 1507.36 & 0.65940 & 7.043 \\
\hline 3600.496 & 0.35380 & 1065 & 10 & 1594.13 & 0.67995 & 9.226 \\
\hline 3603.995 & 0.27080 & 864 & 10 & 1478.69 & 0.67715 & 9.987 \\
\hline 3607.529 & 0.36130 & 1237 & 6 & 1523.75 & 0.62298 & 6.321 \\
\hline 3610.583 & 0.41930 & 1036 & 1 & 922.00 & 0.67435 & 1.608 \\
\hline 3614.707 & 0.31430 & 855 & 10 & 1538.01 & 0.65707 & 9.895 \\
\hline 3620.586 & 0.35300 & 897 & 10 & 1471.64 & 0.69396 & 9.792 \\
\hline 3625.299 & 0.30670 & 813 & 7 & 1637.33 & 0.64446 & 6.634 \\
\hline 3629.747 & 0.36580 & 883 & 3 & 1618.81 & 0.68042 & 2.724 \\
\hline 3633.144 & 0.35200 & 3309 & 10 & 2013.79 & 0.60803 & 8.167 \\
\hline 3633.650 & 0.32050 & 3250 & 4 & 2572.89 & 0.60056 & 2.589 \\
\hline 3635.174 & 0.37110 & 846 & 7 & 1408.19 & 0.64399 & 7.719 \\
\hline 3639.981 & 0.43700 & 1217 & 10 & 1498.49 & 0.61037 & 10.933 \\
\hline 3643.462 & 0.30400 & 1106 & 8 & 1233.31 & 0.67201 & 9.653 \\
\hline 3644.296 & 0.36130 & 801 & 9 & 1723.31 & 0.66501 & 7.853 \\
\hline 3651.632 & 0.28750 & 1199 & 3 & 1456.51 & 0.63792 & 3.229 \\
\hline 3656.068 & 0.33300 & 1096 & 5 & 1646.01 & 0.62765 & 4.840 \\
\hline 3660.475 & 0.39140 & 1180 & 6 & 1486.46 & 0.64306 & 6.277 \\
\hline 3685.245 & 0.24330 & 3232 & 1 & 334.64 & 0.63886 & 4.678 \\
\hline 3685.671 & 0.53980 & 836 & 7 & 1435.58 & 0.62625 & 7.786 \\
\hline 3685.698 & 0.24560 & 3229 & 0 & 111.34 & 0.65614 & 0.000 \\
\hline 3685.802 & 0.30740 & 2253 & 1 & 120.23 & 0.64680 & 12.859 \\
\hline
\end{tabular}




\begin{tabular}{|c|c|c|c|c|c|c|}
\hline \multicolumn{4}{|c|}{$\gamma \gamma$ candidate events, for $\left|\cos \theta^{*}\right| \leq 0.20$} & \multicolumn{3}{|c|}{ Continued from previous page } \\
\hline $\begin{array}{c}\text { CM energy } \\
\sqrt{s} \\
(\mathrm{MeV})\end{array}$ & $\begin{array}{c}\text { Energy spread } \\
\sigma_{E} \\
(\mathrm{MeV})\end{array}$ & $\begin{array}{l}\text { 1st run } \\
\text { number }\end{array}$ & $N_{e v t}$ & $\begin{array}{l}\text { Luminosity } \\
\qquad \mathrm{cm}^{-2} \mathrm{sec}^{-1}\end{array}$ & $\begin{array}{c}\text { Efficiency } \\
\epsilon\end{array}$ & $\begin{array}{l}\sigma^{0.20} \\
(\mathrm{pb})\end{array}$ \\
\hline 3685.982 & 0.25650 & 3226 & 1 & 213.12 & 0.63372 & 7.404 \\
\hline 3686.042 & 0.25860 & 2249 & 0 & 183.15 & 0.65240 & 0.000 \\
\hline 3686.191 & 0.28170 & 2247 & 0 & 115.73 & 0.64913 & 0.000 \\
\hline 3686.209 & 0.48700 & 877 & 6 & 1085.27 & 0.67388 & 8.204 \\
\hline 3686.242 & 0.41810 & 1006 & 2 & 1095.08 & 0.68135 & 2.680 \\
\hline 3686.245 & 0.24860 & 3223 & 0 & 213.23 & 0.63979 & 0.000 \\
\hline 3686.342 & 0.29320 & 2245 & 1 & 260.30 & 0.62158 & 6.181 \\
\hline 3686.381 & 0.28140 & 2242 & 1 & 308.34 & 0.66921 & 4.846 \\
\hline 3686.505 & 0.26310 & 3218 & 2 & 306.72 & 0.65100 & 10.016 \\
\hline 3686.614 & 0.32350 & 2239 & 0 & 198.87 & 0.66501 & 0.000 \\
\hline 3686.647 & 0.32030 & 2235 & 0 & 272.21 & 0.66454 & 0.000 \\
\hline 3686.785 & 0.28140 & 3213 & 2 & 326.14 & 0.65100 & 9.420 \\
\hline 3686.810 & 0.29310 & 2221 & 2 & 477.36 & 0.62485 & 6.705 \\
\hline 3687.027 & 0.28560 & 2218 & 2 & 318.74 & 0.63559 & 9.872 \\
\hline 3687.293 & 0.28820 & 3210 & 0 & 320.02 & 0.63979 & 0.000 \\
\hline 3726.465 & 0.30210 & 3235 & 4 & 979.68 & 0.62905 & 6.491 \\
\hline 3858.457 & 0.47220 & 3329 & 1 & 880.18 & 0.57581 & 1.973 \\
\hline 4289.526 & 0.51810 & 3323 & 0 & 754.54 & 0.46747 & 0.000 \\
\hline
\end{tabular}


Table E.2: $\gamma \gamma$ candidate events, for $\left|\cos \theta^{*}\right| \leq 0.40$.

\begin{tabular}{|c|c|c|c|c|c|c|}
\hline $\begin{array}{c}\text { CM energy } \\
\sqrt{s} \\
(\mathrm{MeV}) \\
\end{array}$ & $\begin{array}{c}\text { Energy spread } \\
\sigma_{E} \\
(\mathrm{MeV}) \\
\end{array}$ & $\begin{array}{l}\text { 1st run } \\
\text { number }\end{array}$ & $N_{e v t}$ & $\begin{array}{l}\text { Luminosity } \\
\qquad \int \mathcal{L} \\
\mathrm{cm}^{-2} \sec ^{-1}\end{array}$ & $\begin{array}{c}\text { Efficiency } \\
\epsilon\end{array}$ & $\begin{array}{l}\sigma^{0.40} \\
(\mathrm{pb})\end{array}$ \\
\hline 2911.365 & 0.28610 & 2324 & 225 & 514.68 & 0.72273 & 604.881 \\
\hline 2912.259 & 0.24760 & 2295 & 92 & 190.21 & 0.76448 & 632.687 \\
\hline 2930.109 & 0.22000 & 2102 & 106 & 317.63 & 0.73581 & 453.546 \\
\hline 2930.482 & 0.17010 & 2042 & 132 & 372.28 & 0.73114 & 484.961 \\
\hline 2930.625 & 0.18170 & 2107 & 79 & 224.41 & 0.73599 & 478.313 \\
\hline 2930.753 & 0.25560 & 2330 & 261 & 855.31 & 0.70209 & 434.636 \\
\hline 2950.177 & 0.29460 & 1136 & 87 & 261.60 & 0.71049 & 468.081 \\
\hline 2950.710 & 0.18510 & 2098 & 128 & 406.10 & 0.72030 & 437.586 \\
\hline 2950.774 & 0.26960 & 2225 & 189 & 604.88 & 0.68182 & 458.271 \\
\hline 2951.131 & 0.27060 & 2175 & 119 & 373.33 & 0.74879 & 425.692 \\
\hline 2956.259 & 0.18000 & 2309 & 119 & 397.91 & 0.73954 & 404.389 \\
\hline 2956.290 & 0.24020 & 2314 & 281 & 979.22 & 0.69256 & 414.351 \\
\hline 2966.866 & 0.23780 & 2259 & 170 & 587.86 & 0.70003 & 413.101 \\
\hline 2966.937 & 0.34740 & 1255 & 88 & 285.76 & 0.71143 & 432.863 \\
\hline 2970.145 & 0.17930 & 2086 & 120 & 401.54 & 0.73553 & 406.308 \\
\hline 2973.136 & 0.30220 & 2273 & 226 & 788.67 & 0.70284 & 407.718 \\
\hline 2973.970 & 0.21510 & 2052 & 170 & 619.80 & 0.69583 & 394.180 \\
\hline 2975.388 & 0.22740 & 2147 & 81 & 344.59 & 0.69798 & 336.775 \\
\hline 2980.475 & 0.17450 & 1299 & 116 & 440.73 & 0.72665 & 362.20 s \\
\hline 2980.513 & 0.27870 & 1291 & 81 & 249.94 & 0.72945 & 444.274 \\
\hline 2983.052 & 0.28540 & 2359 & 290 & 1004.18 & 0.70937 & 407.110 \\
\hline 2985.989 & 0.18930 & 2139 & 119 & 467.67 & 0.72123 & 352.802 \\
\hline 2986.086 & 0.30270 & 2344 & 396 & 1410.42 & 0.68210 & 411.62 \\
\hline 2987.575 & 0.27530 & 1271 & 99 & 341.00 & 0.72525 & 400.306 \\
\hline 2987.749 & 0.19320 & 1093 & 60 & 249.58 & 0.72871 & 329.905 \\
\hline
\end{tabular}




\begin{tabular}{|c|c|c|c|c|c|c|}
\hline \multicolumn{4}{|c|}{$\gamma \gamma$ candidate events, for $\left|\cos \theta^{*}\right| \leq 0.40$} & \multicolumn{3}{|c|}{ Continued from previous page } \\
\hline $\begin{array}{c}\text { CM energy } \\
\sqrt{s} \\
(\mathrm{MeV})\end{array}$ & $\begin{array}{c}\text { Energy spread } \\
\sigma_{E} \\
(\mathrm{MeV})\end{array}$ & $\begin{array}{l}\text { 1st run } \\
\text { number }\end{array}$ & $N_{e v t}$ & $\begin{array}{l}\text { Luminosity } \\
\qquad \mathcal{L} \\
\mathrm{cm}^{-2} \mathrm{sec}^{-1}\end{array}$ & $\begin{array}{c}\text { Efficiency } \\
\epsilon\end{array}$ & $\begin{array}{l}\sigma^{0.40} \\
(\mathrm{pb})\end{array}$ \\
\hline 2987.998 & 0.22880 & 2188 & 115 & 370.71 & 0.73020 & 424.836 \\
\hline 2990.660 & 0.24090 & 2162 & 141 & 499.08 & 0.72450 & 389.949 \\
\hline 2996.140 & 0.20240 & 2065 & 193 & 742.67 & 0.68854 & 377.424 \\
\hline 3000.857 & 0.25420 & 2155 & 71 & 347.31 & 0.71871 & 284.437 \\
\hline 3001.308 & 0.21170 & 2184 & 43 & 209.76 & 0.72292 & 283.568 \\
\hline 3004.917 & 0.31750 & 2298 & 239 & 1169.54 & 0.70704 & 289.028 \\
\hline 3009.094 & 0.25010 & 2120 & 195 & 929.27 & 0.71012 & 295.502 \\
\hline 3025.170 & 0.18670 & 1193 & 27 & 104.22 & 0.71871 & 360.460 \\
\hline 3026.103 & 0.24110 & 1209 & 78 & 489.33 & 0.71376 & 223.326 \\
\hline 3096.682 & 0.11360 & 3078 & 13 & 151.85 & 0.77522 & 110.434 \\
\hline 3097.068 & 0.16110 & 824 & 47 & 274.52 & 0.74412 & 230.082 \\
\hline 3097.137 & 0.14970 & 908 & 22 & 285.22 & 0.75869 & 101.667 \\
\hline 3097.255 & 0.25810 & 1331 & 53 & 491.92 & 0.74113 & 145.374 \\
\hline 3100.605 & 0.20100 & 3284 & 13 & 143.16 & 0.77148 & 111.771 \\
\hline 3215.712 & 0.36110 & 3206 & 24 & 419.93 & 0.69910 & 81.751 \\
\hline 3269.433 & 0.28740 & 3346 & 16 & 411.76 & 0.75420 & 51.521 \\
\hline 3318.819 & 0.71580 & 3301 & 16 & 951.38 & 0.70377 & 23.897 \\
\hline 3405.813 & 0.47820 & 3278 & 2 & 80.67 & 0.67920 & 36.502 \\
\hline 3406.760 & 0.35630 & 3278 & 23 & 925.95 & 0.67920 & 36.571 \\
\hline 3414.783 & 0.35110 & 3270 & 13 & 584.95 & 0.63419 & 35.044 \\
\hline 3414.819 & 0.19710 & 3265 & 8 & 353.18 & 0.73366 & 30.875 \\
\hline 3418.135 & 0.90030 & 3076 & 3 & 146.25 & 0.74739 & 27.446 \\
\hline 3418.456 & 0.27800 & 3245 & 8 & 692.03 & 0.70396 & 16.422 \\
\hline 3429.533 & 0.58750 & 3316 & 6 & 348.75 & 0.75280 & 22.854 \\
\hline 3429.867 & 0.37780 & 3318 & 6 & 390.24 & 0.77643 & 19.802 \\
\hline 3494.432 & 0.33350 & 3341 & 7 & 502.79 & 0.67977 & 20.481 \\
\hline 3508.590 & 0.23210 & 3201 & 5 & 376.23 & 0.65866 & 20.177 \\
\hline 3509.217 & 0.18080 & 1431 & 4 & 303.97 & 0.72973 & 18.033 \\
\hline 3509.798 & 0.20450 & 1396 & 3 & 235.09 & 0.72871 & 17.512 \\
\hline
\end{tabular}




\begin{tabular}{|c|c|c|c|c|c|c|}
\hline \multicolumn{4}{|c|}{$\gamma \gamma$ candidate events, for $\left|\cos \theta^{*}\right| \leq 0.40$} & \multicolumn{3}{|c|}{ Continued from previous page } \\
\hline $\begin{array}{c}\text { CM energy } \\
\sqrt{s} \\
(\mathrm{MeV})\end{array}$ & $\begin{array}{c}\text { Energy spread } \\
\sigma_{E} \\
(\mathrm{MeV})\end{array}$ & $\begin{array}{l}\text { 1st run } \\
\text { number }\end{array}$ & $N_{e v t}$ & $\begin{array}{l}\text { Luminosity } \\
\qquad \mathrm{cm}^{-2} \sec ^{-1}\end{array}$ & $\begin{array}{c}\text { Efficiency } \\
\epsilon\end{array}$ & $\begin{array}{l}\sigma^{0.40} \\
(\mathrm{pb})\end{array}$ \\
\hline 3509.932 & 0.25200 & 3198 & 3 & 317.08 & 0.66109 & 14.312 \\
\hline 3510.362 & 0.27850 & 3194 & 6 & 314.95 & 0.64605 & 29.488 \\
\hline 3510.405 & 0.27650 & 1318 & 11 & 1125.03 & 0.68210 & 14.334 \\
\hline 3510.539 & 0.24610 & 1427 & 3 & 314.50 & 0.70097 & 13.608 \\
\hline 3510.748 & 0.28990 & 3191 & 3 & 318.76 & 0.63531 & 14.814 \\
\hline 3510.779 & 0.25750 & 3286 & 9 & 780.02 & 0.69209 & 16.671 \\
\hline 3510.831 & 0.29490 & 3295 & 17 & 1147.06 & 0.62690 & 23.641 \\
\hline 3511.053 & 0.30790 & 3188 & 5 & 319.43 & 0.63811 & 24.530 \\
\hline 3511.172 & 0.21370 & 1394 & 2 & 218.01 & 0.73814 & 12.428 \\
\hline 3511.445 & 0.31510 & 3185 & 5 & 315.47 & 0.64474 & 24.583 \\
\hline 3511.752 & 0.20990 & 1424 & 4 & 319.68 & 0.68649 & 18.227 \\
\hline 3513.002 & 0.30390 & 3181 & 1 & 301.33 & 0.64306 & 5.161 \\
\hline 3524.642 & 0.30440 & 3081 & 52 & 3716.90 & 0.63839 & 21.915 \\
\hline 3525.161 & 0.28020 & 3037 & 31 & 2902.98 & 0.64353 & 16.594 \\
\hline 3525.505 & 0.28230 & 3103 & 44 & 3531.91 & 0.63017 & 19.769 \\
\hline 3525.637 & 0.34600 & 1414 & 24 & 1800.85 & 0.65473 & 20.355 \\
\hline 3525.680 & 0.29210 & 2444 & 48 & 3477.46 & 0.66314 & 20.815 \\
\hline 3525.782 & 0.28230 & 3060 & 36 & 2976.09 & 0.65025 & 18.603 \\
\hline 3525.800 & 0.28120 & 3136 & 49 & 3821.46 & 0.62279 & 20.588 \\
\hline 3525.860 & 0.24440 & 3053 & 20 & 1196.30 & 0.66781 & 25.034 \\
\hline 3526.093 & 0.41110 & 1306 & 18 & 1985.59 & 0.66706 & 13.590 \\
\hline 3526.105 & 0.29510 & 3005 & 36 & 2291.40 & 0.65800 & 23.877 \\
\hline 3526.134 & 0.28410 & 1383 & 20 & 1864.73 & 0.65399 & 16.400 \\
\hline 3526.162 & 0.29490 & 3016 & 41 & 3308.60 & 0.64371 & 19.251 \\
\hline 3526.515 & 0.28170 & 3162 & 41 & 3233.74 & 0.63671 & 19.913 \\
\hline 3526.520 & 0.31690 & 2404 & 12 & 975.65 & 0.65912 & 18.660 \\
\hline 3526.610 & 0.29620 & 2439 & 10 & 649.03 & 0.64371 & 23.936 \\
\hline 3526.841 & 0.38100 & 1403 & 18 & 1983.64 & 0.64306 & 14.111 \\
\hline 3526.891 & 0.25590 & 2409 & 36 & 3094.13 & 0.64558 & 18.022 \\
\hline
\end{tabular}




\begin{tabular}{|c|c|c|c|c|c|c|}
\hline \multicolumn{4}{|c|}{$\gamma \gamma$ candidate events, for $\left|\cos \theta^{*}\right| \leq 0.40$} & \multicolumn{3}{|c|}{ Continued from previous page } \\
\hline $\begin{array}{c}\text { CM energy } \\
\sqrt{s} \\
(\mathrm{MeV})\end{array}$ & $\begin{array}{c}\text { Energy spread } \\
\sigma_{E} \\
(\mathrm{MeV})\end{array}$ & $\begin{array}{l}\text { 1st run } \\
\text { number }\end{array}$ & $N_{e v t}$ & $\begin{array}{l}\text { Luminosity } \\
\qquad \mathrm{cm}^{-2} \sec ^{-1}\end{array}$ & $\begin{array}{c}\text { Efficiency } \\
\epsilon\end{array}$ & $\begin{array}{l}\sigma^{0.40} \\
(\mathrm{pb})\end{array}$ \\
\hline 3527.491 & 0.21500 & 2426 & 16 & 1396.10 & 0.66267 & 17.294 \\
\hline 3529.099 & 0.40230 & 2387 & 28 & 2328.05 & 0.67061 & 17.935 \\
\hline 3535.451 & 0.53280 & 1244 & 12 & 1303.89 & 0.63587 & 14.474 \\
\hline 3544.786 & 0.25290 & 774 & 8 & 997.54 & 0.73814 & 10.865 \\
\hline 3554.655 & 0.37800 & 1379 & 9 & 489.76 & 0.64698 & 28.403 \\
\hline 3555.164 & 0.28940 & 769 & 12 & 519.35 & 0.70657 & 32.701 \\
\hline 3555.575 & 0.39120 & 1374 & 9 & 411.00 & 0.64558 & 33.920 \\
\hline 3555.922 & 0.33010 & 2202 & 12 & 516.41 & 0.65315 & 35.578 \\
\hline 3555.989 & 0.32990 & 2376 & 26 & 994.19 & 0.64521 & 40.533 \\
\hline 3556.078 & 0.40830 & 1371 & 10 & 413.36 & 0.64539 & 37.484 \\
\hline 3556.180 & 0.29520 & 762 & 11 & 521.27 & 0.69676 & 30.286 \\
\hline 3556.326 & 0.33690 & 2197 & 14 & 752.35 & 0.63260 & 29.416 \\
\hline 3556.645 & 0.33930 & 2192 & 24 & 810.30 & 0.63092 & 46.945 \\
\hline 3556.761 & 0.42570 & 1368 & 10 & 400.58 & 0.65735 & 37.976 \\
\hline 3557.302 & 0.31030 & 750 & 14 & 868.78 & 0.70638 & 22.813 \\
\hline 3557.931 & 0.43910 & 1365 & 8 & 382.85 & 0.64941 & 32.177 \\
\hline 3555.259 & 0.32530 & 3339 & 12 & 501.02 & 0.66828 & 35.840 \\
\hline 3556.060 & 0.31550 & 1086 & 22 & 778.22 & 0.74954 & 37.716 \\
\hline 3576.054 & 0.25090 & 1283 & 13 & 1605.78 & 0.63447 & 12.760 \\
\hline 3580.087 & 0.36050 & 1132 & 9 & 582.80 & 0.67014 & 23.044 \\
\hline 3580.869 & 0.31880 & 1122 & 7 & 626.96 & 0.61906 & 18.036 \\
\hline 3585.185 & 0.35770 & 1186 & 13 & 1505.67 & 0.64521 & 13.382 \\
\hline 3590.246 & 0.35550 & 1112 & 18 & 1483.73 & 0.70610 & 17.181 \\
\hline 3595.616 & 0.33580 & 1126 & 14 & 1507.36 & 0.65754 & 14.125 \\
\hline 3600.496 & 0.35380 & 1065 & 20 & 1594.13 & 0.68883 & 18.214 \\
\hline 3603.995 & 0.27080 & 864 & 21 & 1478.69 & 0.68649 & 20.687 \\
\hline 3607.529 & 0.36130 & 1237 & 14 & 1523.75 & 0.63484 & 14.473 \\
\hline 3610.583 & 0.41930 & 1036 & 2 & 922.00 & 0.68089 & 3.186 \\
\hline 3614.707 & 0.31430 & 855 & 31 & 1538.01 & 0.66127 & 30.481 \\
\hline
\end{tabular}




\begin{tabular}{|c|c|c|c|c|c|c|}
\hline \multicolumn{4}{|c|}{$\gamma \gamma$ candidate events, for $\left|\cos \theta^{*}\right| \leq 0.40$} & \multicolumn{3}{|c|}{ Continued from previous page } \\
\hline $\begin{array}{c}\text { CM energy } \\
\sqrt{s} \\
(\mathrm{MeV})\end{array}$ & $\begin{array}{c}\text { Energy spread } \\
\sigma_{E} \\
(\mathrm{MeV})\end{array}$ & $\begin{array}{l}\text { 1st run } \\
\text { number }\end{array}$ & $N_{e v t}$ & $\begin{array}{l}\text { Luminosity } \\
\qquad \int \mathcal{L} \\
\mathrm{cm}^{-2} \sec ^{-1}\end{array}$ & $\begin{array}{c}\text { Efficiency } \\
\epsilon\end{array}$ & $\begin{array}{l}\sigma^{0.40} \\
(\mathrm{pb})\end{array}$ \\
\hline 3620.586 & 0.35300 & 897 & 18 & 1471.64 & 0.69863 & 17.507 \\
\hline 3625.299 & 0.30670 & 813 & 17 & 1637.33 & 0.65380 & 15.881 \\
\hline 3629.747 & 0.36580 & 883 & 15 & 1618.81 & 0.68714 & 13.485 \\
\hline 3633.144 & 0.35200 & 3309 & 22 & 2013.79 & 0.60850 & 17.953 \\
\hline 3633.650 & 0.32050 & 3250 & 13 & 2572.89 & 0.60757 & 8.316 \\
\hline 3635.174 & 0.37110 & 846 & 18 & 1408.19 & 0.65165 & 19.615 \\
\hline 3639.981 & 0.43700 & 1217 & 20 & 1498.49 & 0.61709 & 21.628 \\
\hline 3643.462 & 0.30400 & 1106 & 20 & 1233.31 & 0.68341 & 23.729 \\
\hline 3644.296 & 0.36130 & 801 & 23 & 1723.31 & 0.66921 & 19.943 \\
\hline 3651.632 & 0.28750 & 1199 & 8 & 1456.51 & 0.64119 & 8.566 \\
\hline 3656.068 & 0.33300 & 1096 & 8 & 1646.01 & 0.63699 & 7.630 \\
\hline 3660.475 & 0.39140 & 1180 & 20 & 1486.46 & 0.64773 & 20.772 \\
\hline 3685.245 & 0.24330 & 3232 & 1 & 334.64 & 0.65287 & 4.577 \\
\hline 3685.671 & 0.53980 & 836 & 15 & 1435.58 & 0.62858 & 16.623 \\
\hline 3685.698 & 0.24560 & 3229 & 1 & 111.34 & 0.66594 & 13.487 \\
\hline 3685.802 & 0.30740 & 2253 & 1 & 120.23 & 0.66193 & 12.565 \\
\hline 3685.982 & 0.25650 & 3226 & 1 & 213.12 & 0.64287 & 7.299 \\
\hline 3686.042 & 0.25860 & 2249 & 3 & 183.15 & 0.66155 & 24.760 \\
\hline 3686.191 & 0.28170 & 2247 & 0 & 115.73 & 0.66407 & 0.000 \\
\hline 3686.209 & 0.48700 & 877 & 20 & 1085.27 & 0.68509 & 26.900 \\
\hline 3686.242 & 0.41810 & 1006 & 5 & 1095.08 & 0.68901 & 6.627 \\
\hline 3686.245 & 0.24860 & 3223 & 1 & 213.23 & 0.64698 & 7.249 \\
\hline 3686.342 & 0.29320 & 2245 & 2 & 260.30 & 0.63512 & 12.098 \\
\hline 3686.381 & 0.28140 & 2242 & 6 & 308.34 & 0.67790 & 28.705 \\
\hline 3686.505 & 0.26310 & 3218 & 3 & 306.72 & 0.65847 & 14.854 \\
\hline 3686.614 & 0.32350 & 2239 & 2 & 198.87 & 0.67949 & 14.801 \\
\hline 3686.647 & 0.32030 & 2235 & 5 & 272.21 & 0.67360 & 27.269 \\
\hline 3686.785 & 0.28140 & 3213 & 5 & 326.14 & 0.65847 & 23.283 \\
\hline 3686.810 & 0.29310 & 2221 & 6 & 477.36 & 0.64166 & 19.589 \\
\hline
\end{tabular}




\begin{tabular}{|c|c|c|c|c|c|c|}
\hline \multicolumn{4}{|c|}{$\gamma \gamma$ candidate events, for $\left|\cos \theta^{*}\right| \leq 0.40$} & \multicolumn{3}{|c|}{ Continued from previous page } \\
\hline $\begin{array}{c}\text { CM energy } \\
\sqrt{s} \\
(\mathrm{MeV})\end{array}$ & $\begin{array}{c}\text { Energy spread } \\
\sigma_{E} \\
(\mathrm{MeV})\end{array}$ & $\begin{array}{l}\text { 1st run } \\
\text { number }\end{array}$ & $N_{e v t}$ & $\int_{\mathrm{m}^{-2} \mathrm{sec}^{-}} \mathcal{L}$ & $\begin{array}{c}\text { Efficiency } \\
\epsilon\end{array}$ & $\begin{array}{l}\sigma^{0.40} \\
(\mathrm{pb})\end{array}$ \\
\hline 3687.027 & 0.28560 & 2218 & 2 & 318.74 & 0.64184 & 9.776 \\
\hline 3687.293 & 0.28820 & 3210 & 0 & 320.02 & 0.63811 & 0.000 \\
\hline 3726.465 & 0.30210 & 3235 & 9 & 979.68 & 0.63811 & 14.397 \\
\hline 3858.457 & 0.47220 & 3329 & 1 & 880.18 & 0.58347 & 1.947 \\
\hline 4289.526 & 0.51810 & 3323 & 0 & 754.54 & 0.46915 & 0.000 \\
\hline
\end{tabular}

